\title{
Sites of the third millennium bc to the first millennium ad at North Mains, Strathallan, Perthshire
}

\author{
Gordon J Barclay* \\ with contributions by L P D Barnetson, S Bohncke, G Collins, \\ T G Cowie, C B Denston, P Hulme, M Kenworthy, D Lunt, \\ C Pare, R Powers, L Robertson, J C C Romans, J Shirriffs, \\ M Stenhouse, O W Thorpe, R S Thorpe, C R Wickham-Jones, \\ and $\mathrm{A}$ Young and illustrations by $\mathrm{M}$ Birkeland, J S Rideout, and \\ A Townshend
}

\section{CONTENTS}

The Henge 123

The Barrow 189

The Ring-ditches $\quad 243$

Discussion 248

\section{SUMMARY}

Three sites were excavated: a class II henge, a massive round barrow and a pair of ring-ditches.

Five periods of activity were noted on the henge site: I - pre henge-bank activity, including one burial; II - the class II henge, a ditch with an external bank enclosing a timber ring (late third millennium bc); III - burial and ritual/domestic activity, the former associated with food vessels, cinerary urns and a beaker, the latter with beaker material (second millennium bc); IV - in situ cremation and burial (late second/early first millennium bc); V - long grave cemetery (mid/late first millennium ad). A second timber ring, three burials and a number of pits could not be securely related to this sequence. One of the Period III food vessels had contained a cereal-based material.

The barrow covered a substantial area of old land surface (Period II) exhibiting probable cultivation traces which in turn sealed small pits (Period I). The construction of the barrow (Period III) was undertaken in six phases, which include a complex timber substructure (A), a ring-bank (B), a fire set near the top of the mound (D) and a stone capping (F). The mound was largely built of material dug from a surrounding ditch, though large quantities of field-stone and turf were also used. The mound has been dated to the early/mid second millennium bc. Phosphate concentrations suggest that the barrow had covered burials. Two food vessel sherds were incorporated into the lower mound material. A spindle whorl was found in the upper part of the mound. Multiple and single cremation deposits and two inhumations, both with food vessels, one with a disc-bead jet necklace, had been dug into the mound's surface 
or had been incorporated during its building. A large cupmarked slab was found at the barrow's summit.

The two ring-ditches may have enclosed low barrows. A pit containing cremated bone and 'Western Neolithic' pottery dated to the early/mid third millennium bc was cut by ring-ditch 2 .

\section{INTRODUCTION}

The excavation of the three sites at North Mains prior to their destruction by the realignment of the landing strip of Strathallan Aircraft Museum was carried out by the Central Excavation Unit of SDD (Ancient Monuments) in three seasons totalling eleven months in 1978 and 1979. The sites lay on a level terrace of fluvio-glacial origin between the River Earn and the Machany Water at approximately $35 \mathrm{~m}$ above sea level (NGR Henge NN 9280 1633; Barrow NN 9262 1622; Ring-ditches NN 9311 1632; fig 1). The Ochil Hills to the S, the Grampian foothills to the $\mathrm{N}$ and $\mathrm{NE}$ and the Earn-Allan watershed provide a raised horizon around the site.

The reports of each of the three sites are set out separately. In each a description of the evidence as excavated is followed by the specialist contributions and the excavator's discussion. Six specialist reports dealing with two or more of the sites were more conveniently presented separately towards the end. Wickham-Jones and Cowie have also contributed papers comparing the artefact assemblages of the three sites and discussing broader comparanda. The report is concluded by a general discussion by the excavator. The site archive, including a full illustrated description of the features not published below, has been deposited in the National Monuments Record of Scotland. An interim account was circulated (Barclay 1980); substantial changes in interpretation have since occurred.

One set of conventions has been used on all the plans and sections with the exception of fig 44 , the barrow cross-sections, in which, for the sake of clarity, some of the conventions have been changed, and figs 3 and 49. The site conventions are illustrated on fig 2.

\section{The henge}

\section{INTRODUCTION}

The henge lay at the lip of a steep-sided gully in which flows the Machany Water. At its closest, on the SE side, the henge bank was set immediately beside a steep drop of over $10 \mathrm{~m}$ from the plateau to the valley floor. The site was first recorded by RCAMS aerial survey in 1977 (pl 9). Although virtually obliterated by ploughing, the site was still visible on the ground as a slight circular bank between 60 and $65 \mathrm{~m}$ in diameter, surrounding a dish-shaped hollow. On the aerial photograph of the site a dark ring broken at $\mathrm{E}$ and $\mathrm{W}$ and a surrounding faint light ring were interpreted as a ditch and external bank respectively, a conclusion supported by a resistivity survey carried out by John Price of Bradford University, and subsequently proved by excavation. Within the enclosure a ring of dark circular marks concentric with the ditch was clearly visible. Blurred dark marks suggested the presence of other pits inside and outside the enclosed area. The positions of features outwith the bank and ditch were confirmed by the resistivity survey and the excavated area was laid out to allow as many as possible to be investigated. The aerial and resistivity surveys also detected the line of a recent field boundary ditch crossing the site from $\mathrm{W}$ to $E$, passing through the $E$ gap in the enclosure. Local information suggested that an earlier excavation by 'students' had taken place at some time in the past; no evidence of such disturbance was found.

Work on the site began in March 1979, the first season ending in July. A second season of four weeks was mounted in the autumn of the same year. It was intended that the whole of the 


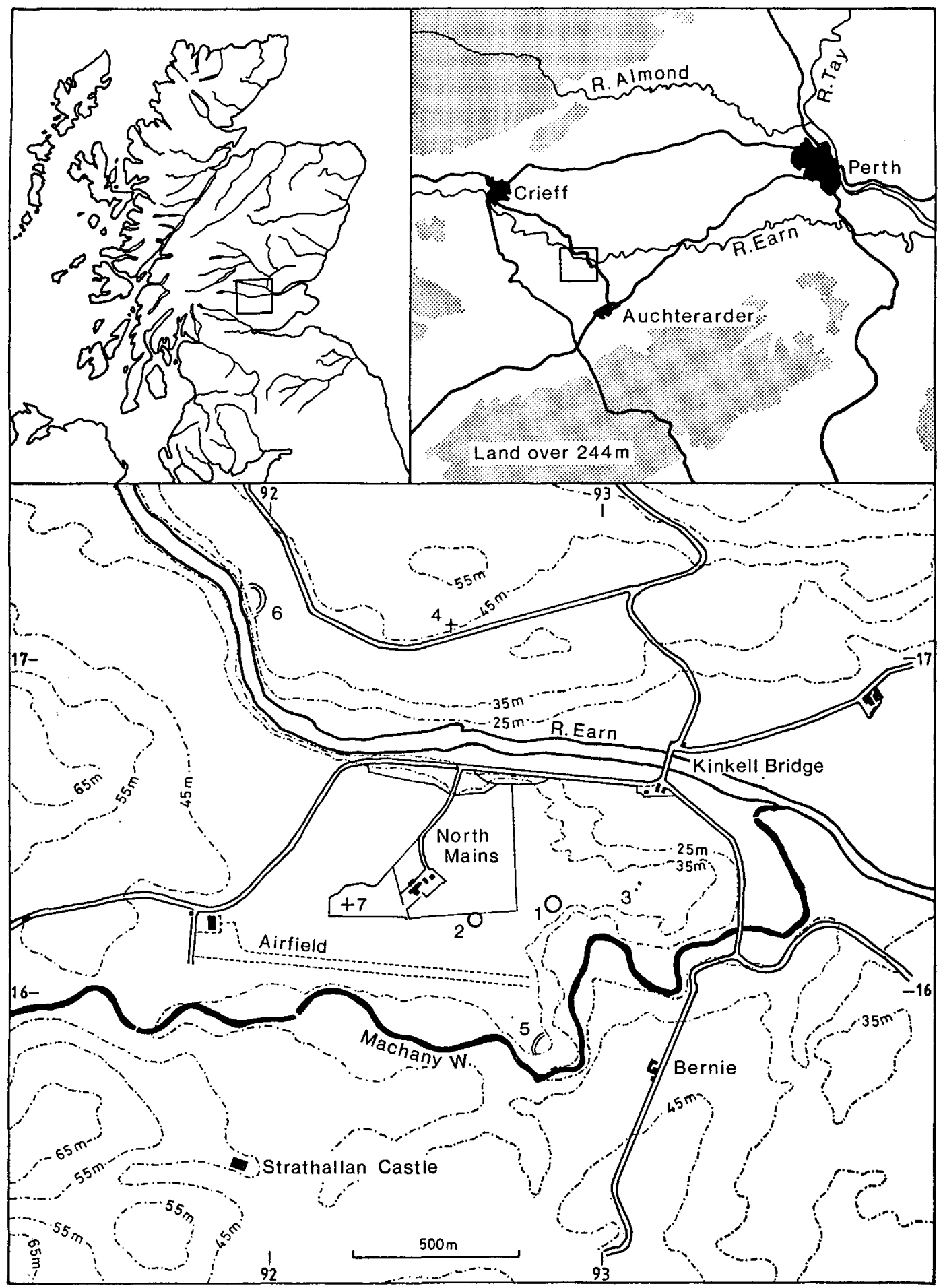

FIg 1 Location maps: 1-Henge, 2-Barrow, 3-Ring-ditches, 4-Site of cairn, 5 and 6-Terrace-edge enclosures, 7 -Site of peat core 


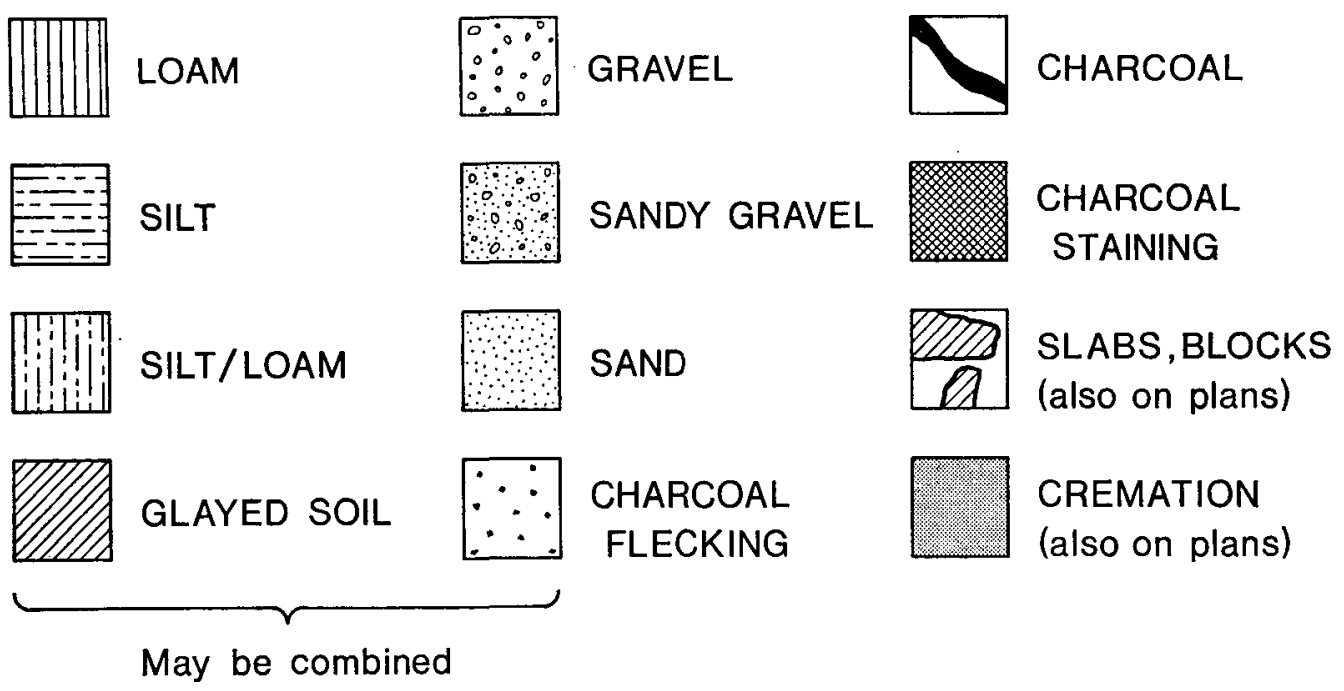

Fig 2 Key to conventions used on all plans and sections except figs 3, 44 and 49

enclosed area would be excavated and, with the exception of a small part of the modern boundary ditch and a possible pit in the $\mathrm{W}$ half, this was achieved. It was planned that the four terminals and sections of the ditch at $\mathrm{N}$ and $\mathrm{S}$ should be excavated, but insufficient time was available for the investigation of the $\mathrm{S}$ section and part of the NE terminal. Areas of bank were investigated to the $\mathrm{S}, \mathrm{W}, \mathrm{N}$ and NE of the ditched enclosure, and to the $\mathrm{N}$ and NE attempts were made to locate the external features noted on the aerial photographs and the resistivity survey. Additionally, at the W entrance, an area was opened to include any features which might lie outside the enclosure or be associated with the gap in the bank and ditch.

The overburden of up to $35 \mathrm{~cm}$ of ploughsoil was removed largely by a mechanical excavator under archaeological supervision to within $5-10 \mathrm{~cm}$ of the subsoil surface, the remainder being removed by hand. All areas were carefully trowel-cleaned repeatedly during the excavation. The subsoil revealed by these processes consisted of fluvio-glacially deposited material ranging in size from very fine silt to coarse gravels. This great variability across the site made the location and excavation of features very difficult. One hundred and twenty-eight of the soil anomalies tested were probably of non-natural origin. Of these, 57 could more or less certainly be assigned to five recognizable periods of activity and their description has been arranged, as far as possible, in chronological order, from Period I to Period V. The remainder, including three burials, probably prehistoric, which could not with any certainty be assigned to a period, are to be found in the Miscellaneous section. Minor features which could not be assigned to a period, provided no evidence for their function, and produced no artefacts, are not illustrated or described. Their position and reference numbers, however, appear on figs 3 and 4 .

\section{DESCRIPTION}

Five main periods of activity were distinguished:

Period I The cultivation of the old land surface, the digging of three pits and the deposition of one cremated body. Third millennium bc. 
Period II The erection of a circle of large timbers (ring A); the digging of the ditch and the erection of the bank; burning on site during the rotting of the timbers. Late third/early second millennium bc.

Period III Burial and ritual/domestic activity associated with a late beaker, food vessels and cinerary urns in the former, and beaker material only in the latter. Early/mid second millennium bc.

Period IV Cremation and interment on the pyre sites to the $\mathrm{N}$ of the enclosure. Late second/ early first millennium bc.

Period V Long graves within the enclosure. Mid to late first millennium ad.

Additionally, an oval setting of 17 pits (ring B), a further 14 features and three burials which could not be assigned with certainty to any period are described after Period V. Their chronological positions are discussed on $\mathrm{p} 188$.

\section{PERIOD I}

The first activity on the site was represented by the fragments of a gleyed fossil topsoil preserved under the Period II henge bank. Even where the bank had recently been ploughed away, the old land surface survived, in places immediately below the modern cultivated soil, although it had been severely damaged. A full consideration of this fossil soil and its agricultural use is presented by Romans and Robertson on p 260. Prior to the erection of the Period II bank, three pits were dug through the surface and subsequently backfilled (F1, F7 and burial A). A fourth feature (F48) was cut by a Period II pit (A/20) and may, therefore, be placed in Period I. Featureless sherds and fragments of coarse pottery, probably from a single vessel, were found under the bank at the S of the site (SF1; SF2). Two further burials, L within the enclosed area and $M$ between the bank and ditch at the N, were similar in style to $A$ and may have been contemporary. The possibility that ring B, an oval setting of pits, belonged to this period is discussed below (p 188). Rather than a single clearly defined episode the few features of Period I probably represent several phases of activity prior to Period II, which could not be distinguished from each other because of the lack of surviving stratigraphy.

\section{Period I features}

F1 (fig 5) This pit, which lay under the Period II henge bank at the NE, measured approximately $1.3 \mathrm{~m}$ long by $1.1 \mathrm{~m}$ broad at the surface, 0.5 by $0.55 \mathrm{~m}$ at the base and was $1.4 \mathrm{~m}$ deep. It had been deliberately backfilled; a few charcoal fragments were recovered from the fill.

F7 (fig 5) This feature, partly sealed by the bank at the $\mathrm{N}$ of the site, measured a maximum of $2.3 \mathrm{~m}$ long, $1.35 \mathrm{~m}$ broad and $0.35 \mathrm{~m}$ deep. Three fills were noted; two silts lining the $\mathrm{N}$ and $\mathrm{S}$ sides were separated by a wedge of gravel which may have been the fill of a partial recut. Owing to difficulties of identifying the subsoil, the bottom of the feature, shown by a dotted line on fig 5 , was overcut during excavation.

Burial A (fig 5) Sealed by the henge bank at the SW of the site was a small scoop which contained the unaccompanied cremated remains of a male human of about 20 years of age (cremated bone catalogue no 1).

F48 (fig 5) This pit was cut by pit 20 of ring A (Period II). It had been over $1.6 \mathrm{~m}$ long and was between 0.6 and $0.8 \mathrm{~m}$ broad and up to $0.38 \mathrm{~m}$ deep. It was filled with a uniform loam containing some stones.

\section{PERIOD II}

The second period of activity on the site saw the erection of a ring of 24 large posts in ramped pits (referred to as ring $\mathrm{A}$ ) and the construction of a massive bank and ditch around it. For reasons which are set out below it seems certain that the post ring was erected before the bank and ditch. 


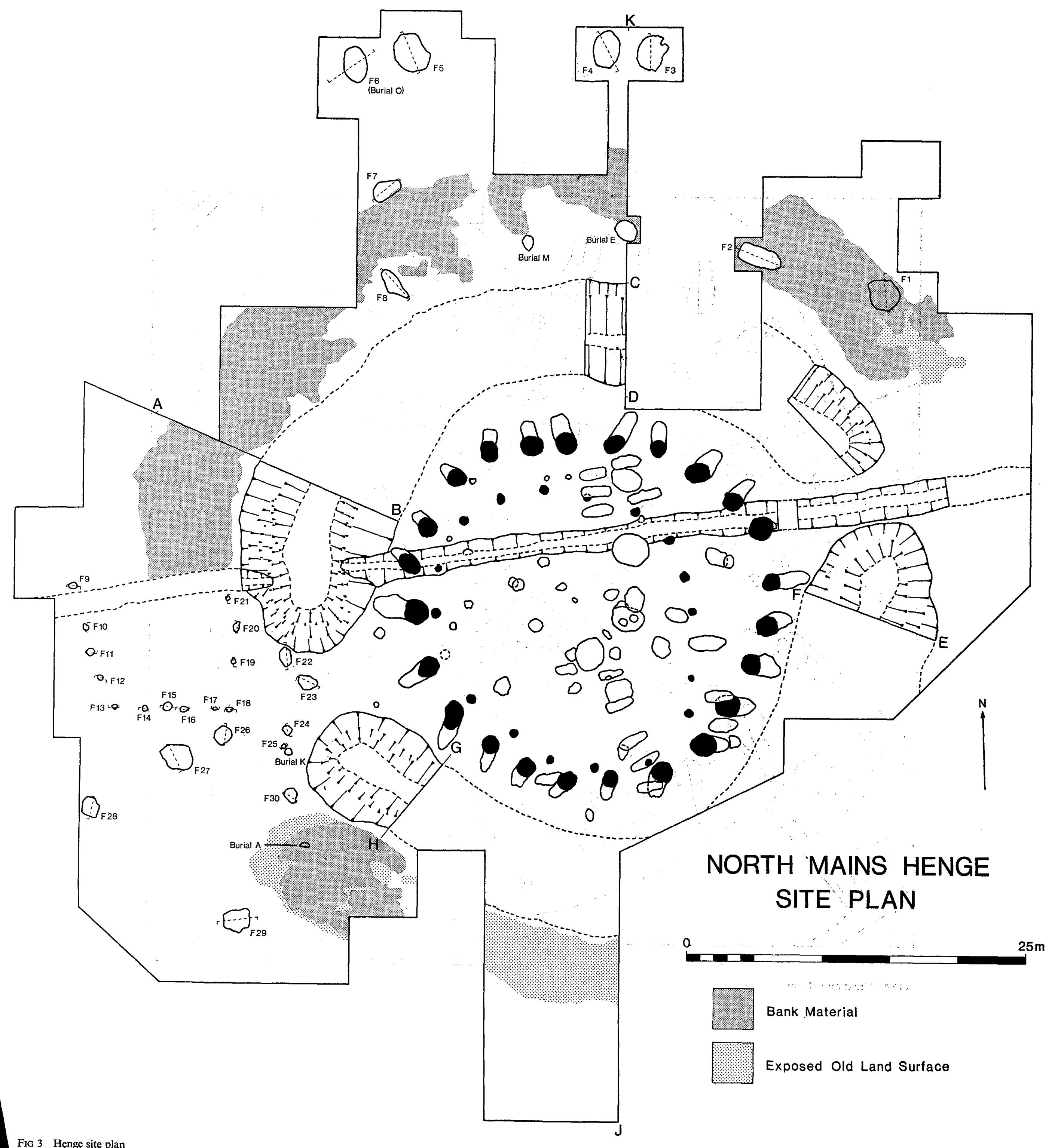




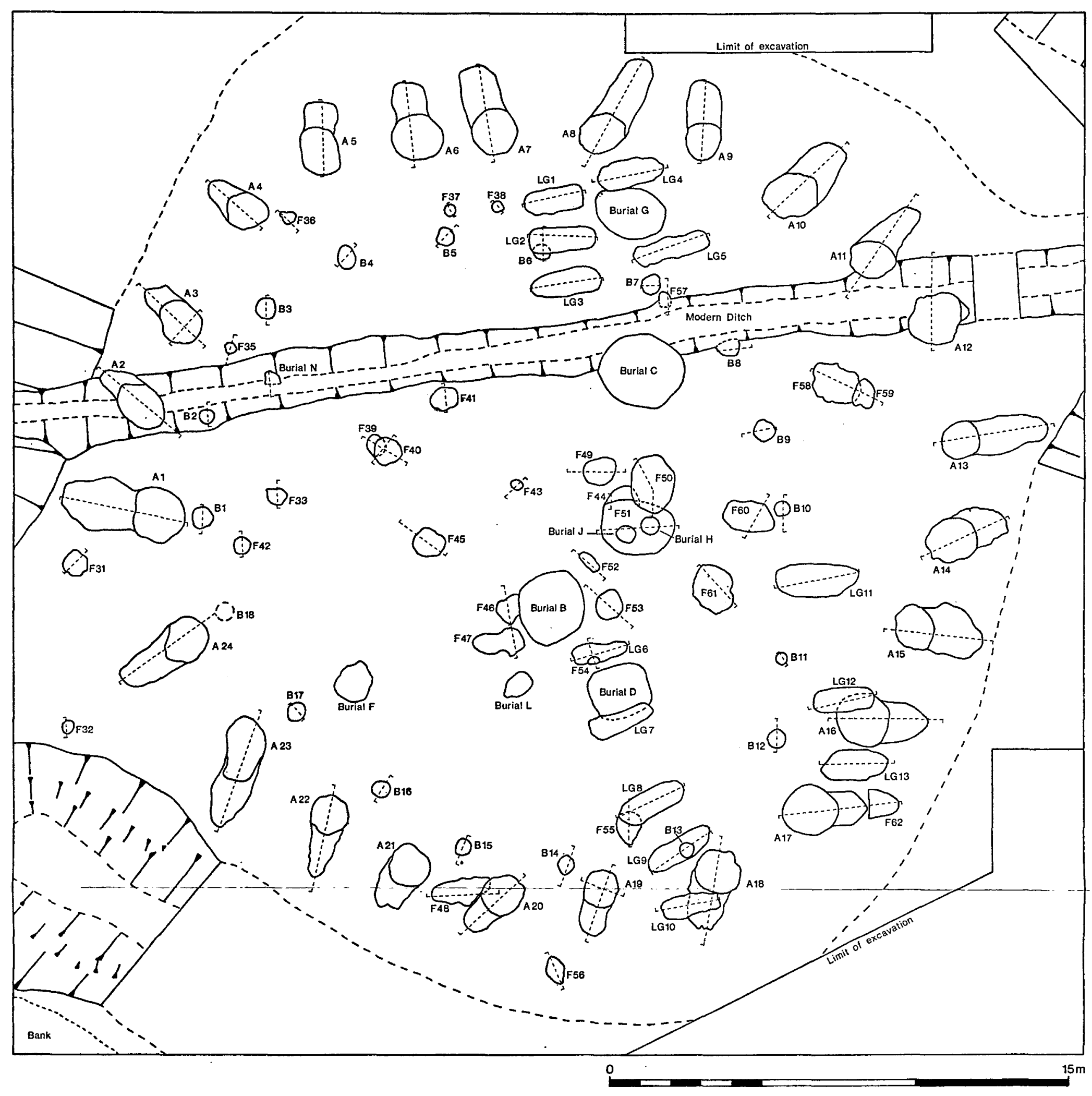

Fro 4 Henge central area 

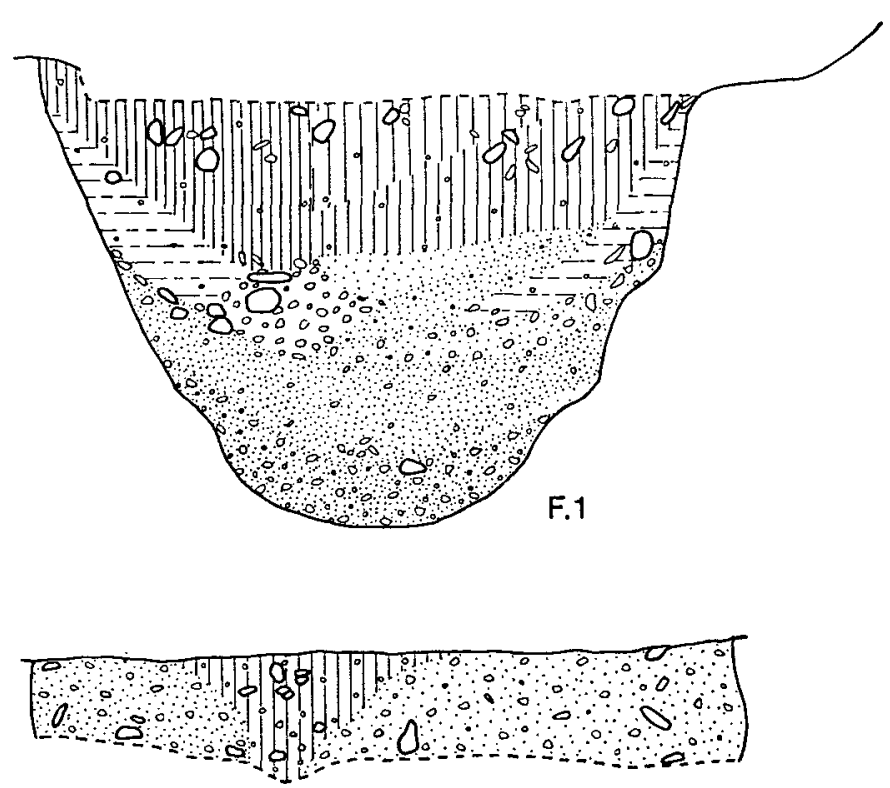

F.7

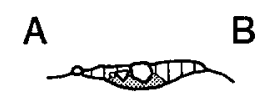<smiles>B1C2CC3CC1C(C1CCCC1)C(C2)C3</smiles>

Burial A

Ring $A 20$
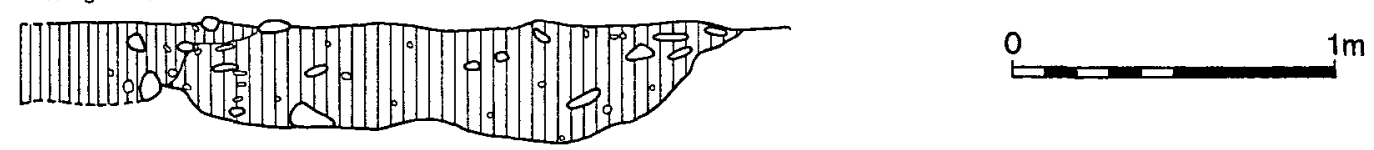

\section{F. 48}

FIG 5 Henge Period I: sections of features

\section{Ring $A$ (figs 4, 6, 7, 8, 9); (Pottery p 155; Struck stone p 168)}

The post pits of the ring were investigated in two ways. Three (A/3, A/19 and A/21) were excavated in quadrants but a lack of light and space in the narrow sections made their excavation and interpretation extremely difficult. The transverse section and a longitudinal profile of $A / 3$ and a profile of $A / 19$ have been included in figs 6 and 9. The remainder were excavated using a single section line over the pit and its ramp, with the exception of $\mathrm{A} / 12$ where modern disturbance had almost completely destroyed the ramp.

The pits were arranged in an uneven circle between 25 and $27 \mathrm{~m}$ in diameter. With few exceptions the pits were set in a series of short straight segments made up of three or four pits (fig 4; table 1). Presumably to aid the erection of the timbers the pits were each provided with a ramp. The ramps consistently pointed out from the ring but were not arranged radially. In some cases the ramps of three or four pits together were roughly parallel and in two, or possibly three, instances radical changes in ramp alignment coincided with the junctions of the straight segments of the ring (eg between $A / 17$ and $A / 18$ (table 1)). There is a marked absence of ramp azimuths in the range $100^{\circ}$ to $190^{\circ}$. The post pits were irregularly spaced, the distance between centres varying from 2.6 to $4.2 \mathrm{~m}$, apparently at random (fig 4; table 1). Their depth varied, also randomly, between 1.35 and $2.05 \mathrm{~m}$, though this might in part be the result of differential surface erosion (fig 10). In two cases marked changes of depth coincide with changes of ramp azimuth: $A / 4$ and $A / 5, A / 17$ and $A / 18$.

In a few cases some material seemed to have been placed or had fallen into the pit before the insertion of the post. The lower fill of A/13 was fine silt which seemed to have 'flowed' into the pit prior to the insertion of the post. It is certain that the silt moved again during the rotting of the post. Pit A/10 may show some deliberate filling prior to the insertion of the post but there is little clear evidence for this and most of the primary fills of the pits seem to have been the result of erosion of the sides before the insertion 


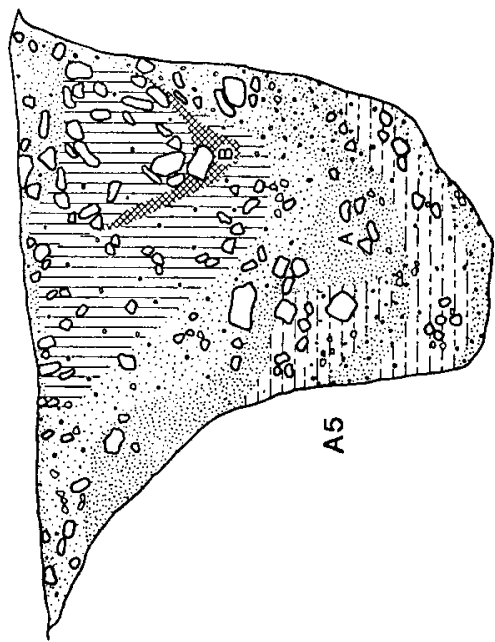

है।
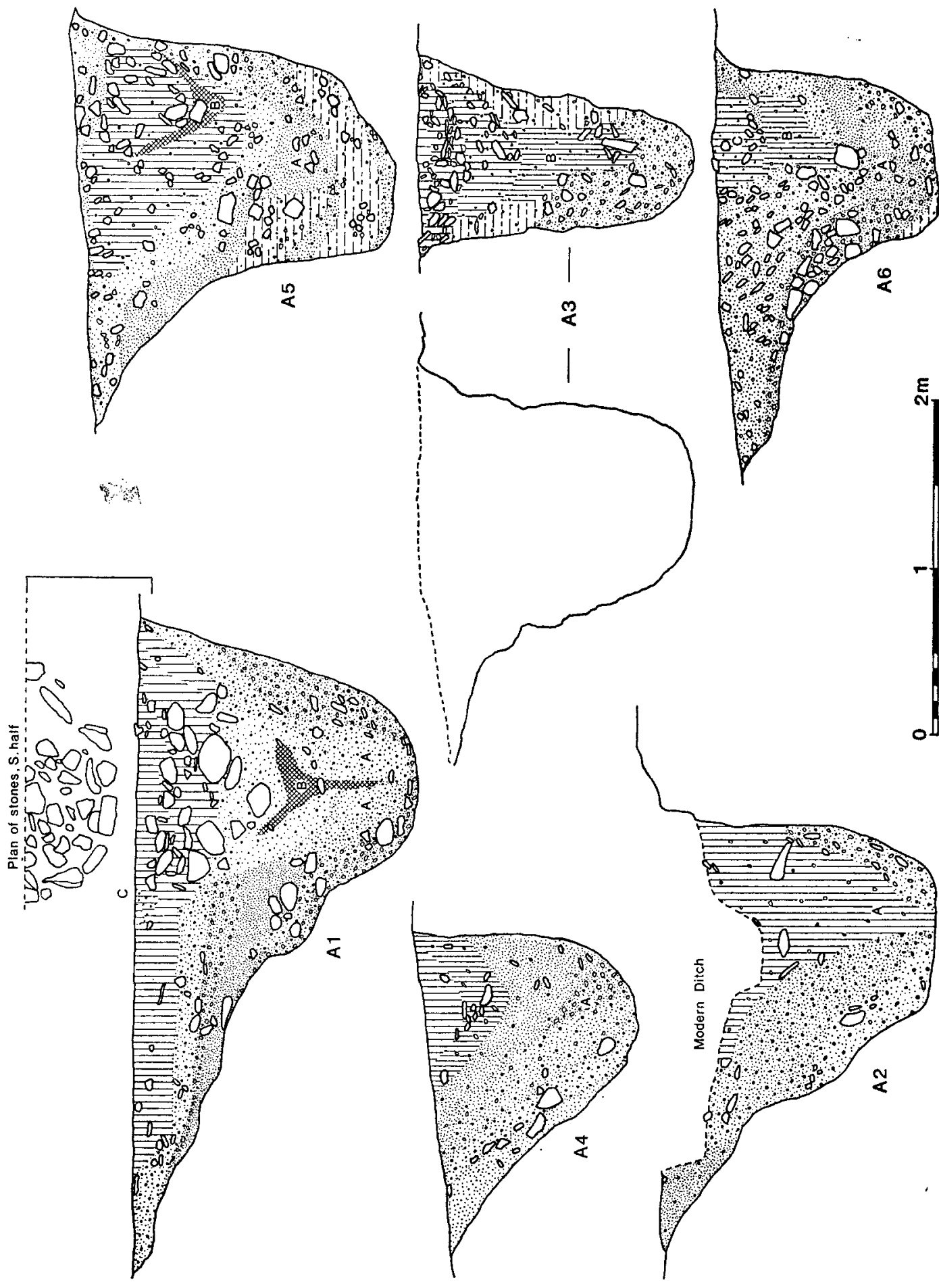

of

?망

8

6
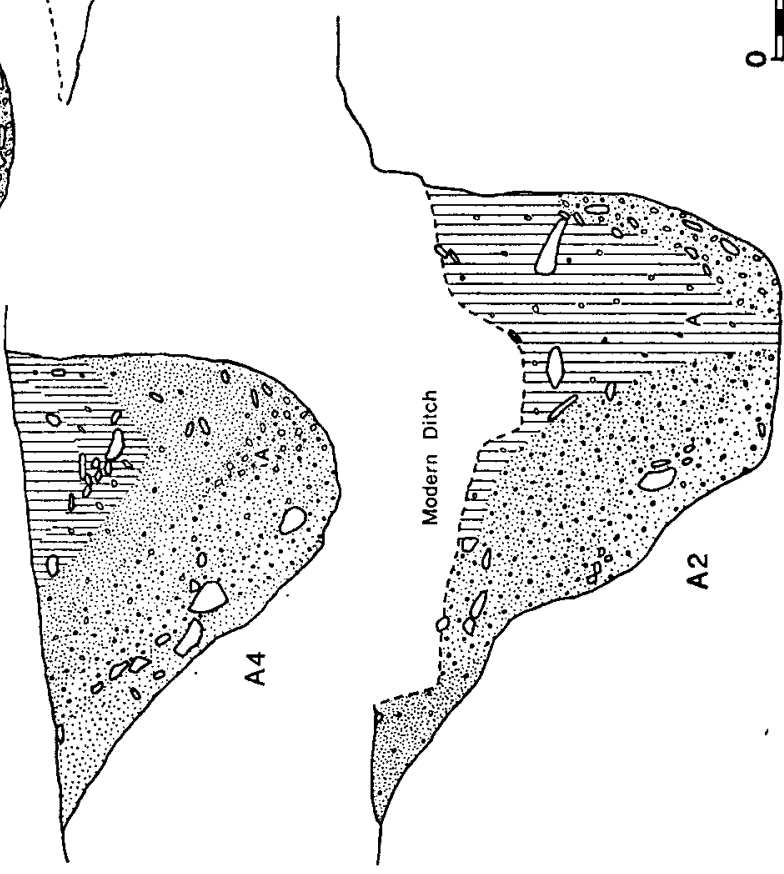

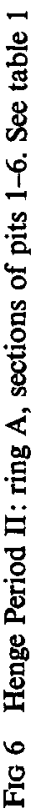




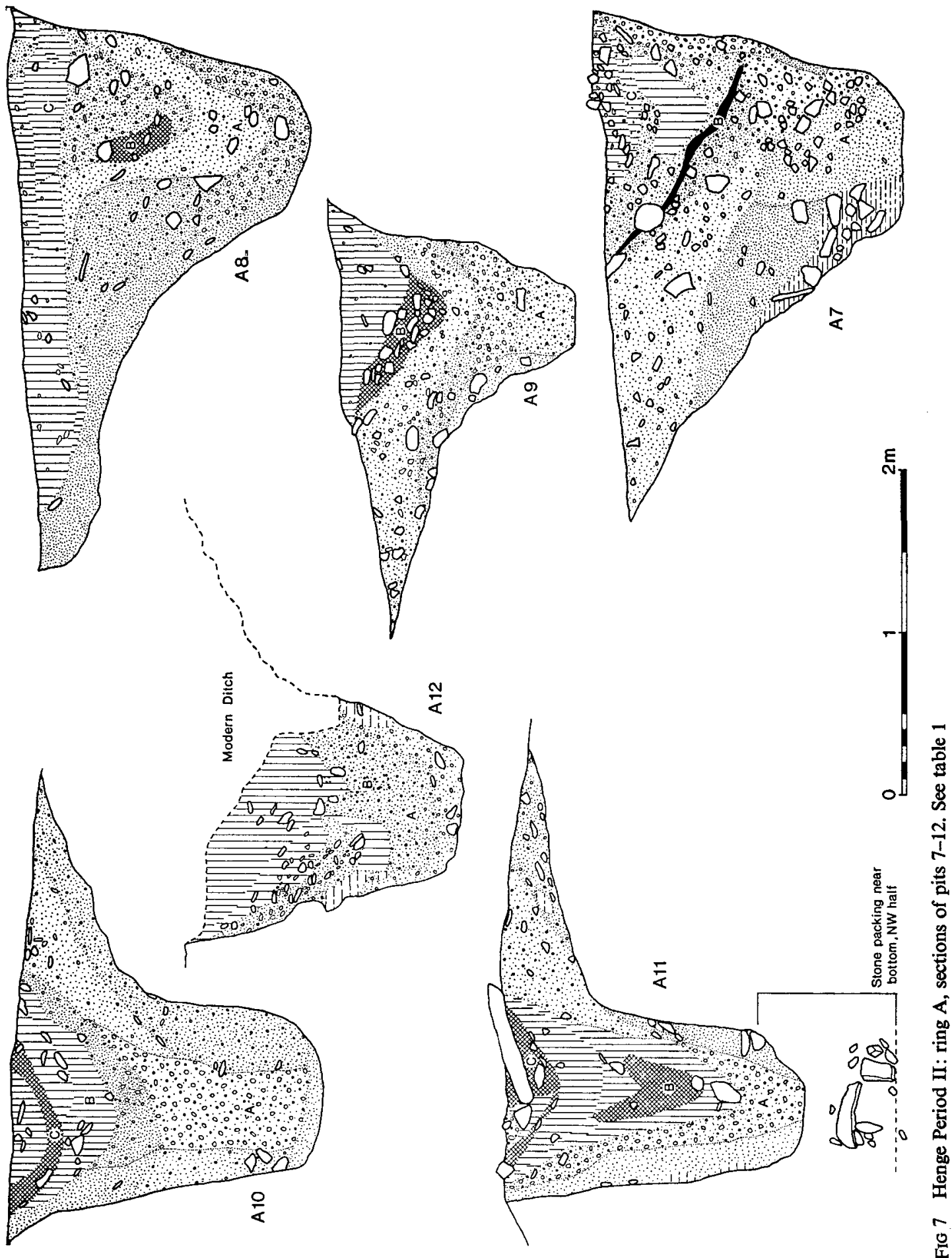


$130 \mid$ SOCIETY OF ANTIQUARIES OF SCOTLAND, 1983
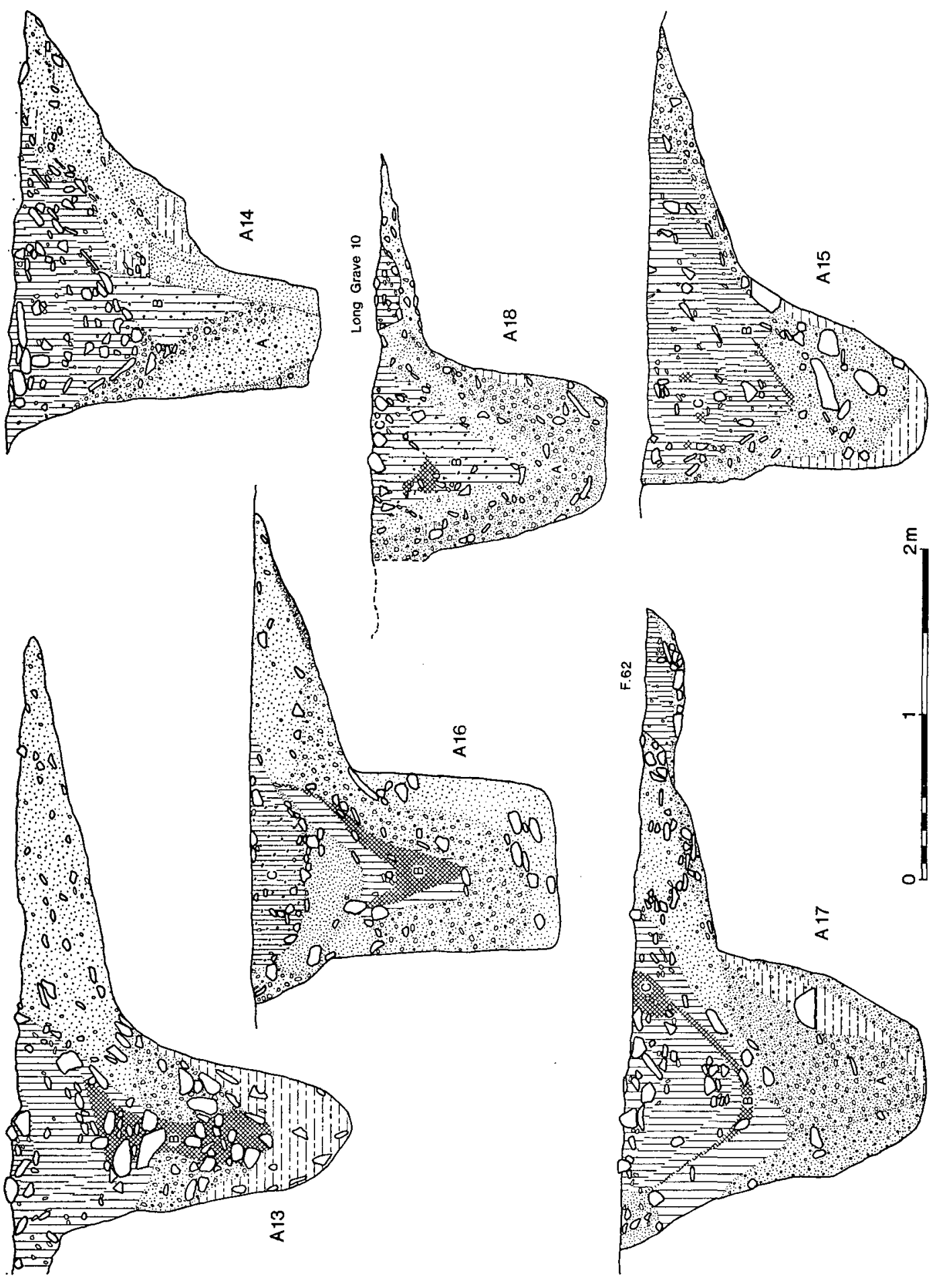

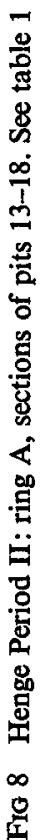



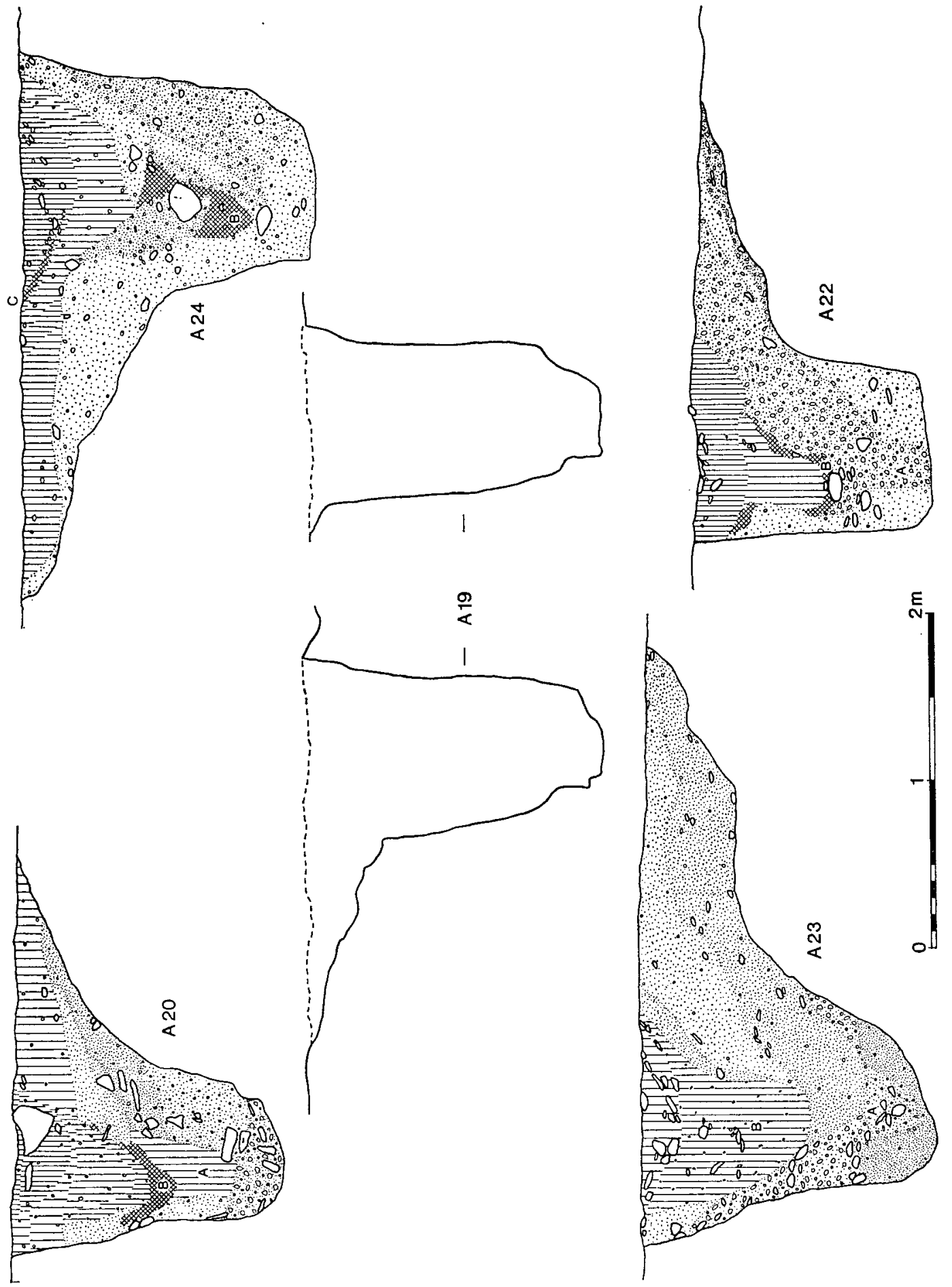

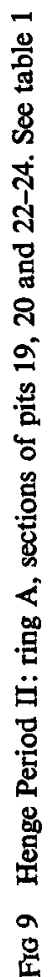


132 | SOCIETY OF ANTIQUARIES OF SCOTLAND, 1983

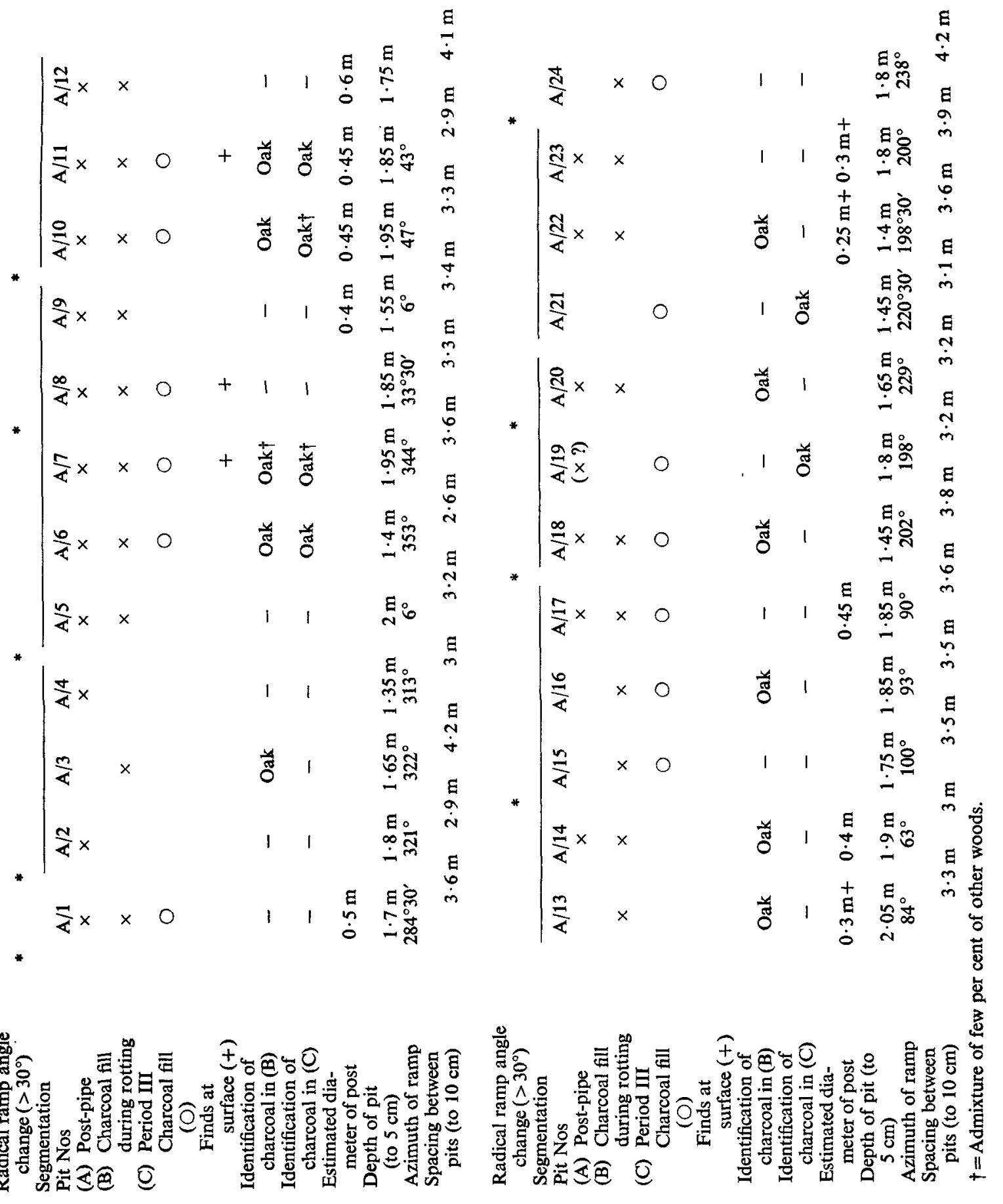


of the posts; the best examples of this are $\mathrm{A} / 6$ and $\mathrm{A} / 15$. Observations carried out after the excavation of the pits suggest that sufficient erosion to account for the material could take place in a matter of days or weeks. Some of the fill may have been dislodged from the sides of the pit during post insertion. Once the posts had been set up in the pits they were packed in position using redeposited gravel, presumably the spoil dug from the pits. Stone was used very infrequently (figs 6,$7 ;$ pits $A / 1, A / 11$ ). Charcoal of oak was found in the primary packing of some of the pits and samples from pits $A / 5$ and $A / 7$ were submitted for radiocarbon dating (2090bc \pm 70 (GU-1354); 2155bc \pm 60 (GU-1353)). The posts seem to have rotted in situ. The large movements of the fills caused by this process at first suggested that there might have been some deliberate disturbance of the pit fills; however, with the possible exception of pit $\mathrm{A} / 15$, this now seems unlikely. The pit fills distorted unevenly as the posts rotted; the extent of the distortion in each pit depended on the particle size of the material used for packing. Therefore, a post-pipe survived in some pits (eg A/10) but in others no trace of one was recorded. Identifiable post-pipes have been marked ' $A$ ' on the section drawings (figs 6-9) and in table 1. The distortion of the post-pipes has prevented an accurate assessment of the diameters of the posts; however, they were probably a maximum of $0.6 \mathrm{~m}$ in diameter.

In 20 of the pits charcoal and charcoal-stained soil were found in the central part of the pit fill, and in the upper part of pit A/7 a burnt plank of oak was found in an equivalent position. These deposits suggested that charcoal had found its way from the surface into the post-centres during the rotting of the heartwood of the timbers and that a substantial area of the henge surface may have been contaminated with charcoal at that time. The possible significance of the burning and the plank are considered below. A sample of the plank in $A / 7$ was submitted for radiocarbon dating (2330bc \pm 60 (GU-1352)). Charcoal samples from equivalent positions in $A / 1$ and $A / 13$ were also dated (respectively $2065 \mathrm{bc} \pm 65$ (GU-1435) and $2180 \mathrm{bc} \pm 60$ (GU-1436)). In the top of 21 of the pits small hollows had formed as a result of the subsidence and compaction of their fills and in 13 of these (fig 12) deposits of charcoal, charcoal-stained soil and burnt bone were found which reflected later activity on the site, described below (Period III). Only two finds of pottery were made in the pit fills not associated with the Period III activity. In A/2 near the surface a single sherd was found (SF4). In A/3 a similar context produced three sherds and a fragment from a single vessel (SF3).

\section{The ditch and bank}

The ditch was also dug and the bank erected during Period II. Near its terminals the ditch was broad and flat bottomed ( $\mathrm{pl} \mathrm{10}$ ) but at the $\mathrm{N}$ section it was almost ' $\mathrm{V}$ ' shaped. It was approximately $11 \mathrm{~m}$ broad at the NW terminal, $7 \mathrm{~m}$ at the NE, $7 \mathrm{~m}$ at the SE, $6 \mathrm{~m}$ at the SW and $7.5 \mathrm{~m}$ at the $\mathrm{N}$ section; the NW and SE terminals appeared to be more bulbous. It varied in depth between 2.2 and $3 \mathrm{~m}$. The excavated parts were smooth bottomed and showed no evidence of segmentation. Only a small part of the ditch was excavated and the calculations of volume are therefore approximations. The total volume has been estimated at $1250 \mathrm{~m}^{3}$.

The bank survived only in fragments (fig 3). Large areas of it had been destroyed by natural erosion, ploughing and possibly deliberate flattening in historic times. Segments of the bank measuring up to $0.3 \mathrm{~m}$ in height were excavated but most surviving fragments were less than $0.1 \mathrm{~m}$ high. It was impossible to estimate the original width of the bank, effectively preventing an estimate of its height, but it survived to a maximum width of $9 \mathrm{~m}$. The original dimensions of the bank ( $\mathrm{p} 181$ ) have been estimated assuming that the ditch was the only quarry and that all the material quarried was used to build the bank.

The ditch enclosed an area between 32 and $35 \mathrm{~m}$ in diameter and the area enclosed by the surviving outer edge of the bank measured in excess of $65 \mathrm{~m}$ in diameter. The bank in its denuded state was cut by only two features, burial E, described below, and F2. At the $\mathrm{N}$ side of the $\mathrm{W}$ entrance the complete destruction of the bank to the $S$ of the modern boundary ditch prevented the clarification of the relationship between the bank and a group of small pits (F9-F21). This roughly rectangular setting of 13 features, probably postholes, produced no dating material. Nothing similar was found elsewhere near the bank.

\section{PERIOD III}

Subsequent to the construction of the Period II monument and in some cases demonstrably later than its decay, a number of different activities took place in and around the enclosure. Generally they were of two types; burial, and the deposition of domestic or ritual debris. The 


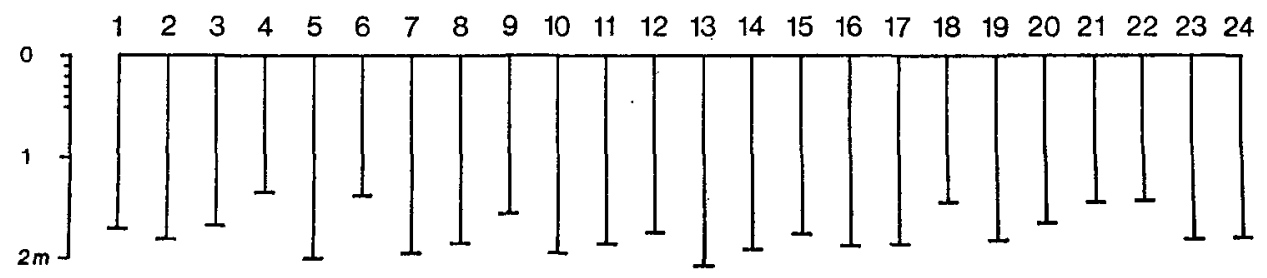

FIo 10, Part 2 Henge Period II: diagram of depths of ring A pits

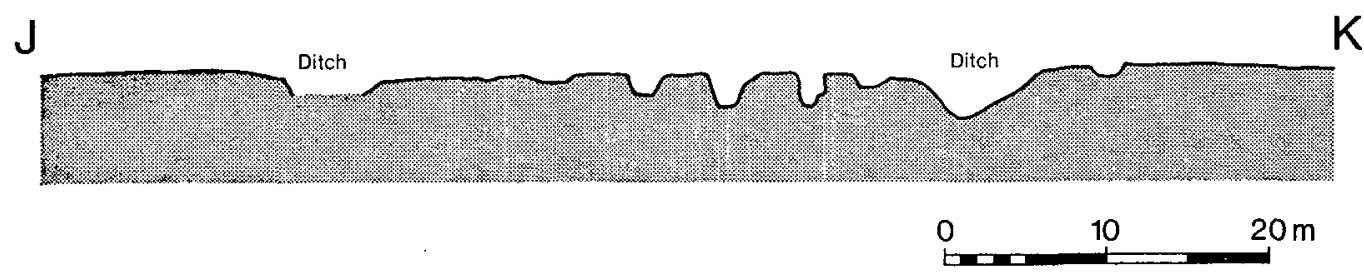

Frg 11 Henge: $\mathrm{N}-\mathrm{S}$ profile (J-K on fig 3)

latter was reflected in the fills of 13 of the hollows formed in the tops of the ring A pits upon the collapse of the post packing, and the fills of one pit found within the enclosure (F33; fig 13). The hollows could have taken a considerable length of time to form and once formed could have survived for many years. The characteristic fill consisted of charcoal, burnt bone or pottery, or a combination of two or all of these. A fragment of carbonized hazelnut shell was found in A/15. Decorated sherds identified as beaker, possibly from one vessel, were found in pits $A / 7$ (SF7) and $\mathrm{A} / 8$, (SF9, 10, 11, 12). Plain beaker sherds were recovered from A/7 (SF6, 8) and A/8 (SF13), while other sherds, from A/8 (SF15) and A/11 (SF5, 14) have tentatively been identified as part of a residual spread of earlier Neolithic pottery. Twelve fragments of baked clay (? daub) were also found in A/7 (SF16). Only one piece of struck stone was found (SF31), in A/8. In A/3 three smoothed stones may have originated in Period III or may, more probably, have been part of the original post packing. The hollows which produced the material are marked ' $\mathrm{C}$ ' in table 1 and on the sections of ring A pits illustrated on figs 6-9. The identifications of the charcoal found in the hollows are also listed in table 1. Many of the fragments of burnt bone recovered from the hollows were too small to allow the identification of the species to which they belonged. However, sufficient survived to allow Barnetson to suggest that large and small artiodactyla were present, possibly cattle, ovicaprids and pigs.

F33 may be associated with these events by the character of its fill, which included charcoal (alder, holly, birch, willow, poplar, hazel), a burnt hazelnut shell and burnt bone of indeterminate species. Flintwork, mostly burnt, (SF34-40) - including a burnt, broken barbed and tanged arrowhead found in the pit - was probably contemporary. The pit was $0.63 \mathrm{~m}$ long, $0.47 \mathrm{~m}$ wide and a maximum of $0.2 \mathrm{~m}$ deep. Its sides were covered by a layer of silt. In the central part was a heavily charcoal-stained soil. None of the hollows in the tops of the ring A pits produced sufficient charcoal to allow a radiocarbon date, but material from $F 33$ was dated to $1450 \mathrm{bc} \pm 60$ (GU-1438).

\section{Burials}

Within the excavated area 29 definite and presumed burials were recorded (figs 3, 4, 12, 21, 23, 24). Most could be related either stratigraphically, by radiocarbon dating, by generally accepted artefact chronologies or by their resemblance to those so dated, to some of the five periods of activity recognized 


\section{$A_{1}$
$n$}

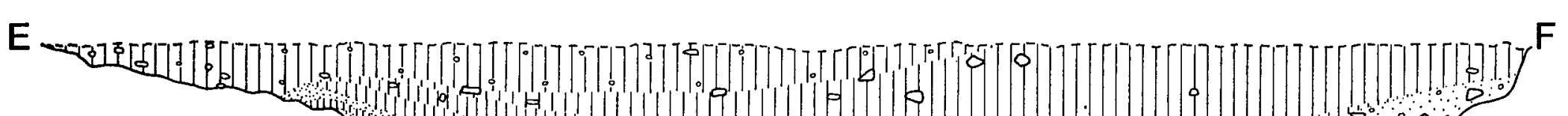
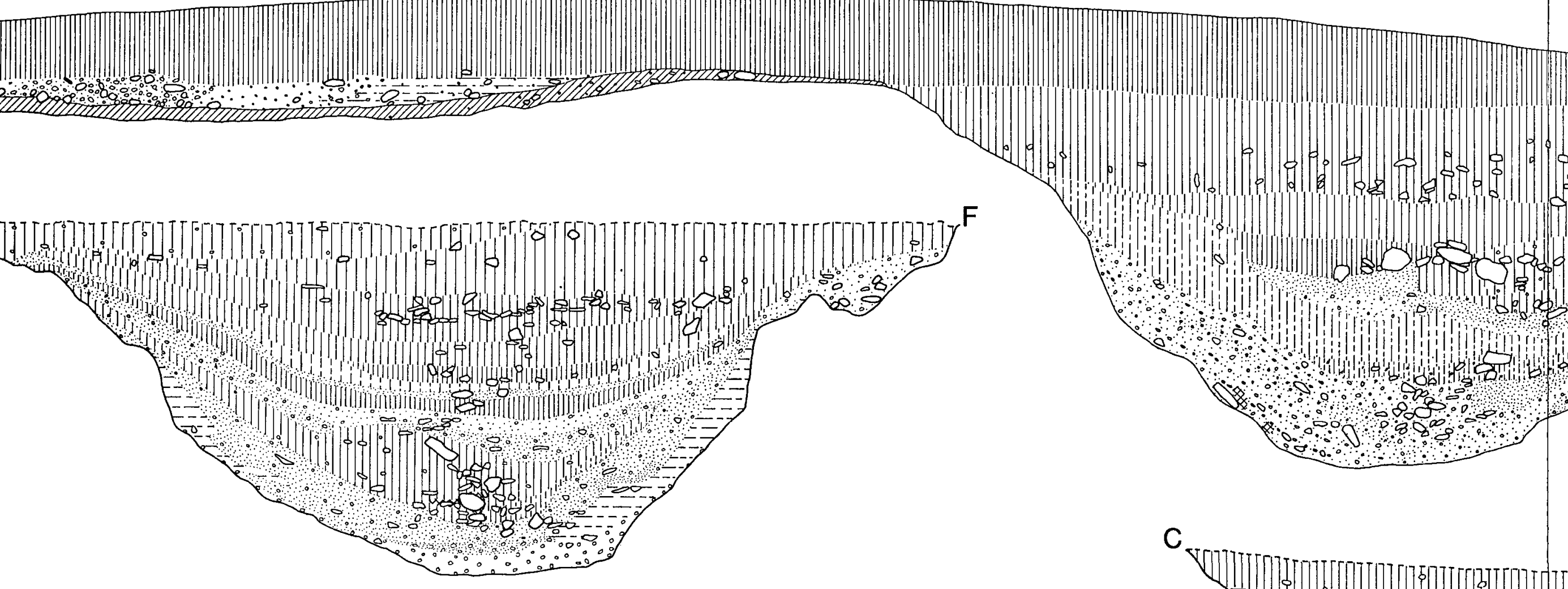

B

G

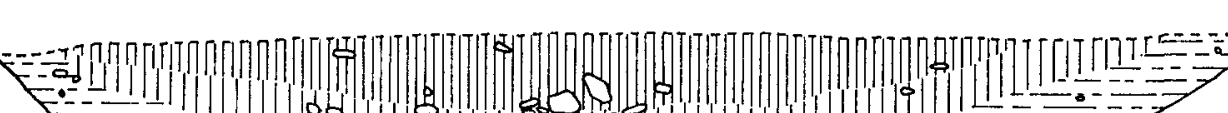

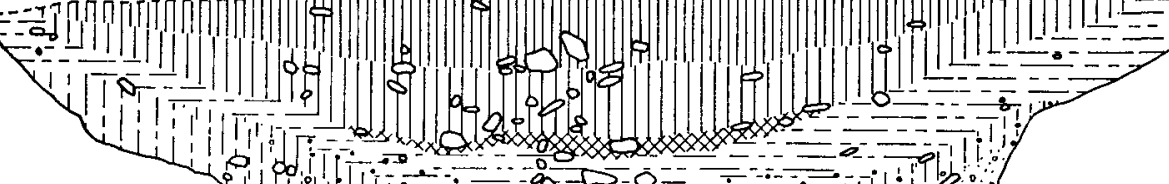

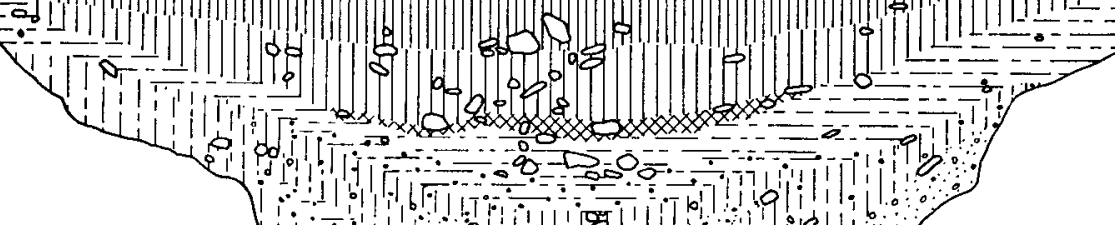

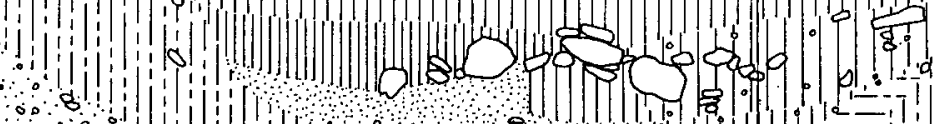

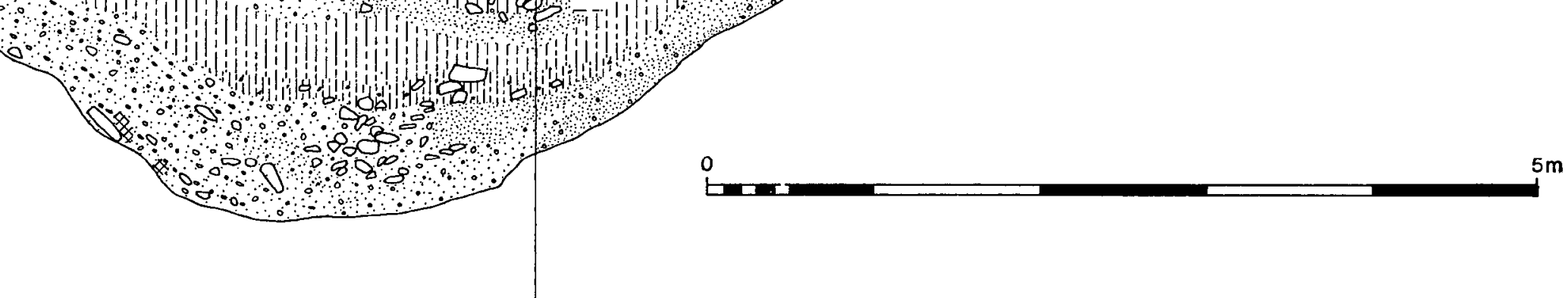

H

$\mathrm{C}_{\mathrm{Y}}$

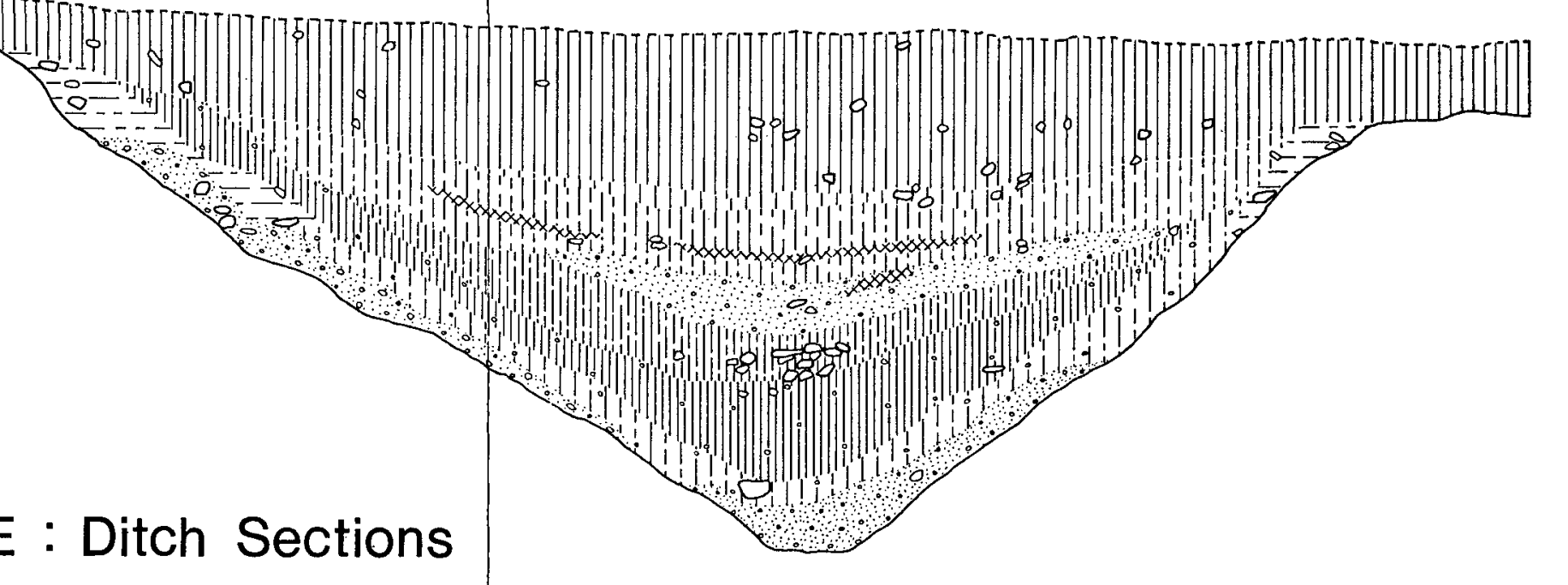




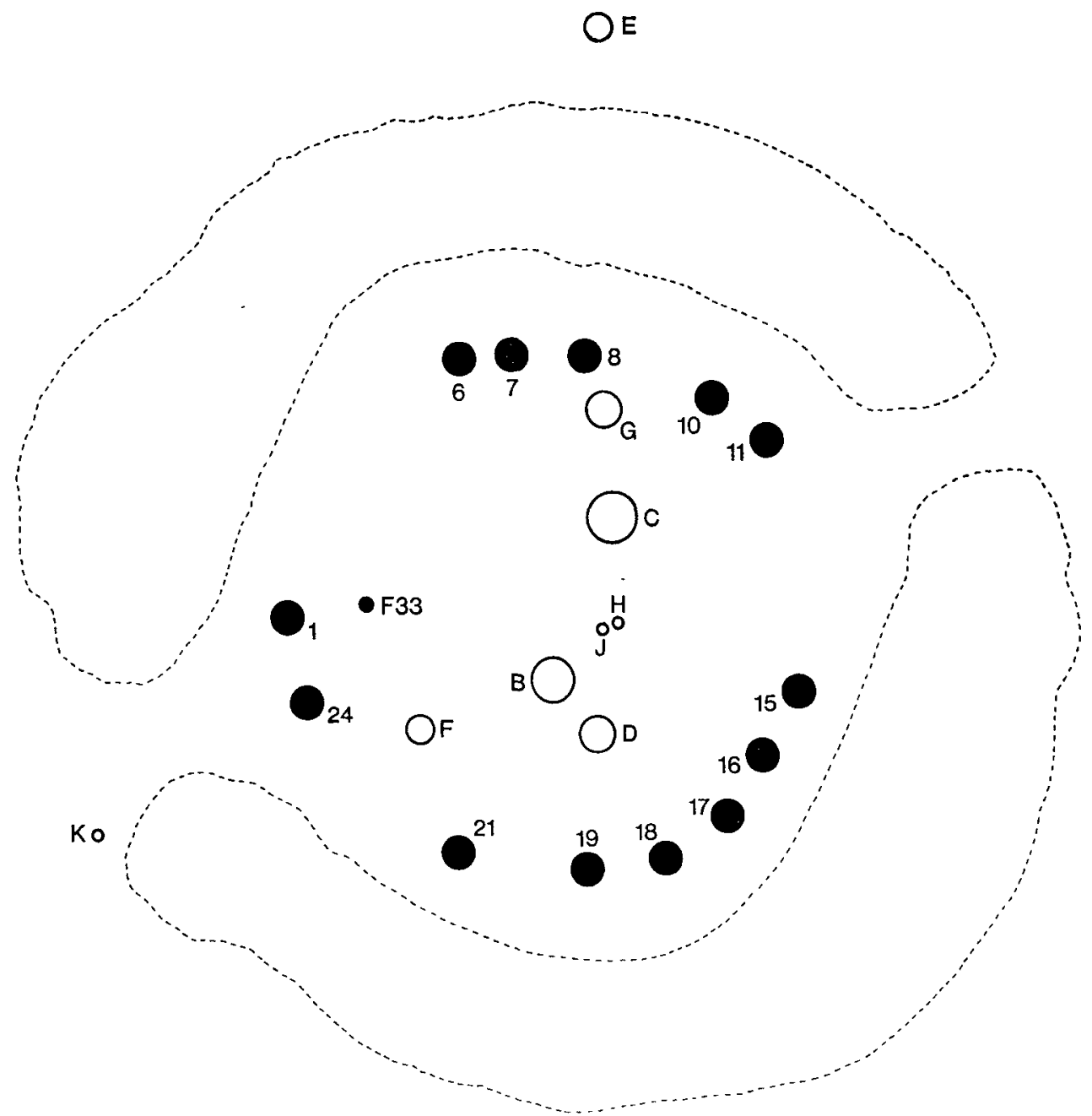

Fig 12 Henge Period III: diagram showing location of features: burials, F33 and deposits in the tops of ring A pits

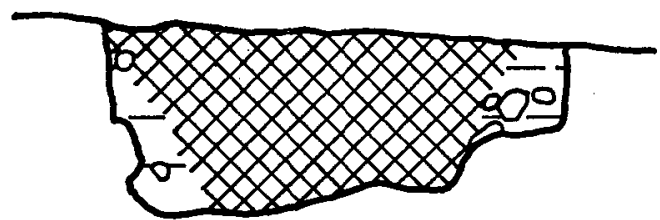

FIg 13 Henge Period III: section of F33 
on the site. Their period assignment, type and associations are summarized on table 2. Nine burials are described below, burials $\mathrm{O}, \mathrm{P}$ and $\mathrm{Q}$ are described in Period $\mathrm{IV}$, while burials $\mathrm{L}, \mathrm{M}$ and $\mathrm{N}$ are described with the Miscellaneous Features (p 145). All dimensions of cists are internal measurements. It should be noted that the burials have been rearranged since the submission of the Balfarg report (Mercer 1981, 164), where they were referred to. The affected burials are as follows: burial A is now B, B is now C and $\mathrm{E}$ is now $F$.

TABLE 2

Burials in and around the henge

\begin{tabular}{|c|c|c|c|c|c|c|c|}
\hline Burial & Inhum/crem & Sex & Age & Interment type & Association & Period & Fig \\
\hline $\begin{array}{l}\text { A } \\
\text { B }\end{array}$ & $\begin{array}{l}\text { Cremation } \\
\text { Inhumation }\end{array}$ & $\begin{array}{l}\text { Male } \\
\text { Female }\end{array}$ & $\begin{array}{l}\text { c20 } \\
20-25\end{array}$ & $\begin{array}{l}\text { Shallow pit } \\
\text { Deep cist }\end{array}$ & $\begin{array}{l}\text { Food vessel } \\
\text { (SF17) }\end{array}$ & III & $\begin{array}{c}5 \\
14,15\end{array}$ \\
\hline $\mathrm{C}$ & ?Inhumation & - & - & Deep cist & & III & 16 \\
\hline D & $\begin{array}{l}\text { ?Crem/ } \\
\text { ?Inhum }\end{array}$ & ?Human + ? & & Shallow cist & $\begin{array}{l}\text { Food vessel } \\
\text { (SF18) }\end{array}$ & III & 17 \\
\hline $\mathbf{E}$ & ?Inhumation & - & - & Shallow pit & $\begin{array}{l}\text { Food vessel } \\
\text { (SF19) } \\
\text { Struck stone } \\
\text { (SF41) }\end{array}$ & III & 18 \\
\hline $\mathbf{F}$ & Cremation & Male & Adult & Shallow cist & $\begin{array}{l}\text { Beaker (SF20) } \\
\text { Struck stone } \\
\text { (SF42-44) }\end{array}$ & III & 17 \\
\hline G & Cremation & Male & ?Under 35 & Deep pit & - & III & 19 \\
\hline $\mathbf{H}$ & Cremation & Male & Adult & Urne & $\begin{array}{l}\text { Collared urn } \\
\text { (SF21) }\end{array}$ & III & pl 1 \\
\hline $\mathbf{J}$ & Cremation & $\begin{array}{l}\text { Male+ }+ \\
\text { child }\end{array}$ & ?Under $35+4-6$ & Urned & $\begin{array}{l}\text { Urn (SF22) } \\
\text { Struck stone } \\
\text { (SF45-48) }\end{array}$ & III & pl 1 \\
\hline $\mathbf{K}$ & Cremation & ?Female & Young adult & Urned & $\begin{array}{l}\text { Enlarged } f \text { v urn } \\
\text { (SF23) }\end{array}$ & III & pl 1 \\
\hline L & Cremation & ?Female & Adult & Shall & - & ? & 24 \\
\hline M & Crem & ?Female & ?c30 & Shallow pit & - & $?$ & \\
\hline$N$ & Cremation & ?Human & ?Adult & Shallow pit & - & $?$ & 24 \\
\hline $\mathbf{O}$ & Cremation & Human & Over 30 & in situ cremation & - & IV & 21 \\
\hline $\begin{array}{l}P \\
O\end{array}$ & Cremation & ?Human & & in situ cremation & - & IV & 一 \\
\hline Q & Cremation & ?Human & & in situ cremation & - & IV & \\
\hline
\end{tabular}

\section{Feature descriptions}

Burial B (figs 14, 15; pl 10) (Bone p 176; Pottery p 155; Palaeobotany p 178)

A large sub-circular pit, between $2.2 \mathrm{~m}$ and $2.6 \mathrm{~m}$ in diameter at the surface, stepped at $0.75 \mathrm{~m}$ below the surface, 1.2 by $0.95 \mathrm{~m}$ at its bottom and $1.6 \mathrm{~m}$ deep, had been dug to receive a cist $\mathrm{c} 1.05 \mathrm{~m}$ long, between $0.45 \mathrm{~m}$ and $0.5 \mathrm{~m}$ wide and $0.65 \mathrm{~m}$ deep, built, using unevenly sized blocks and slabs, on a layer of backfilled gravel. The space behind the cist slabs was backfilled with gravel to the height of their tops and flagstones were placed over the slabs and gravel, providing an even platform for the massive cover slab. The cover was not placed well enough over the cist to avoid leaving gaps (especially on the NE side) and a ring of flags was laid against its edge throughout its circumference (fig 14b). The pit was then completely backfilled with gravel and a layer of flagstones was placed at the level of the modern subsoil surface (fig 14a). No artefacts or trace of any activity were found on this rough paved surface. The cist, containing the relatively well preserved skeleton of a female aged between 20 and 25 years, had remained almost completely free of soil; where soil had penetrated and covered the body no bone had survived (fig $15 \mathrm{a}$ ). The skeleton lay on its right side facing $\mathrm{E}$. The head had apparently been deliberately placed against the $S$ end of the cist so that if faced upwards, across the body's left shoulder. Bone from the skeleton was radiocarbon dated to $1540 \mathrm{bc} \pm 65$ (GU-1381). The burial was accompanied by a food vessel (SF17), which lay on its side in the SE corner of the cist beside the skull (fig 15a). A deposit of organic material found in the pot has been subjected to pollen analysis which revealed that it had probably contained a cereal-based drink or gruel flavoured with meadowsweet. A single fragment of unidentifiable burnt bone was found in the pit above the cover slab, presumably an accidental inclusion in the fill. 


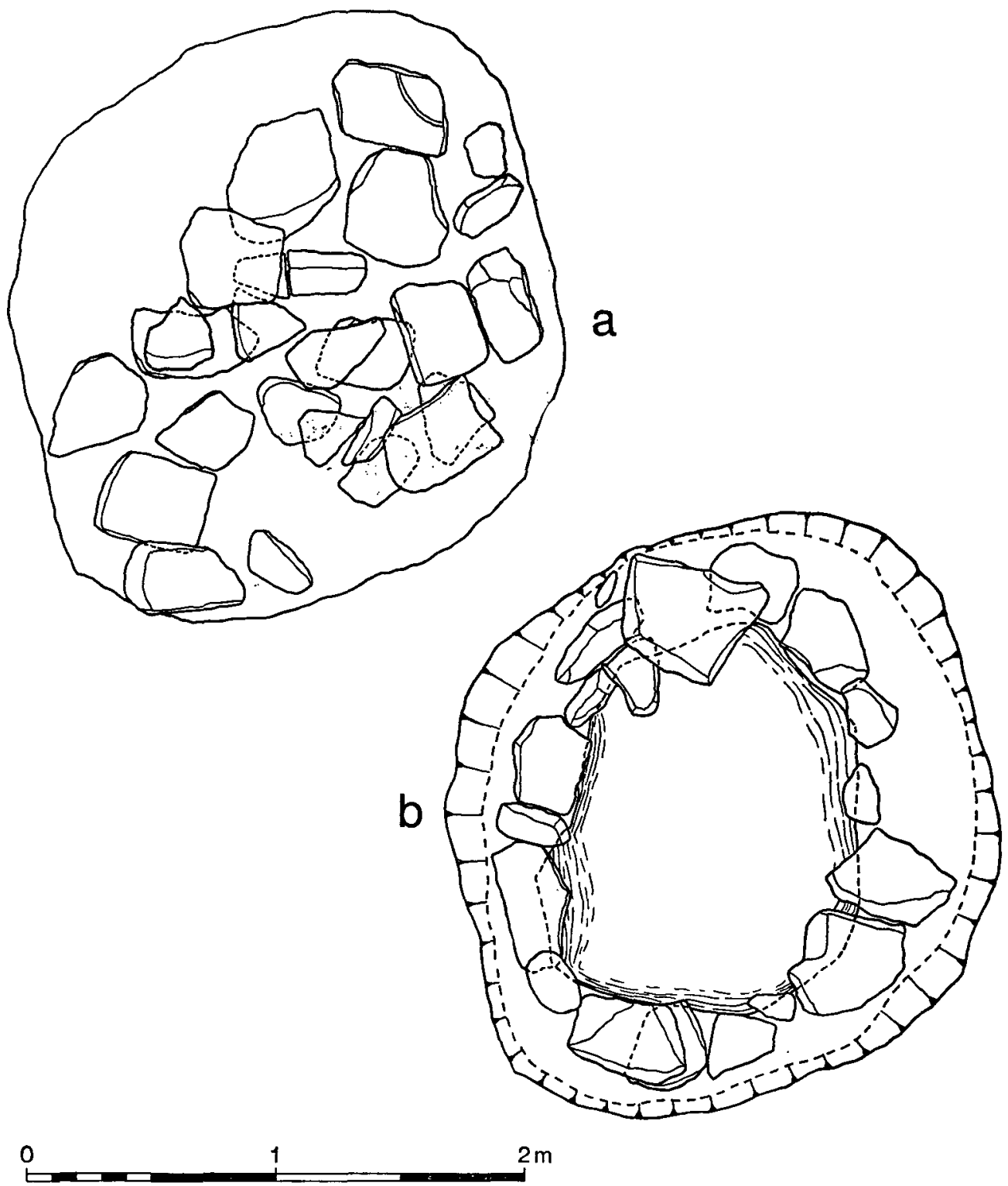

FIg 14 Henge Period III: Burial B: $a$ - paving at surface; $b$ - cover slab and flagging

\section{Burial C (fig 16)}

An almost circular pit c $2.8 \mathrm{~m}$ in diameter at the surface, stepped at $1.2 \mathrm{~m}$ below the surface, $\mathrm{c} 1.35 \mathrm{~m}$ by $1.2 \mathrm{~m}$ at its bottom and $1.9 \mathrm{~m}$ deep, had been dug to receive a cist (c $1 \mathrm{~m}$ long, $0.65 \mathrm{~m}$ wide and 0.55 $0.75 \mathrm{~m}$ deep) built of unevenly sized slabs and blocks. The pit and cist appeared to be more carefully formed than in the similar burial B. As in B the pit was backfilled to the top of the side slabs and flagging was laid on the N, W and S sides. The top of the $\mathrm{E}$ side was higher than the other three. The gravel fills of the cist and of the rest of the pit were indistinguishable from each other and the massive capstone rested entirely on gravel, not touching the side slabs or the flagging at any point (fig 16b). Therefore it seems certain that the pit and cist were backfilled to $c 0 \cdot 15-0.2 \mathrm{~m}$ above the side slabs and flagstones before 

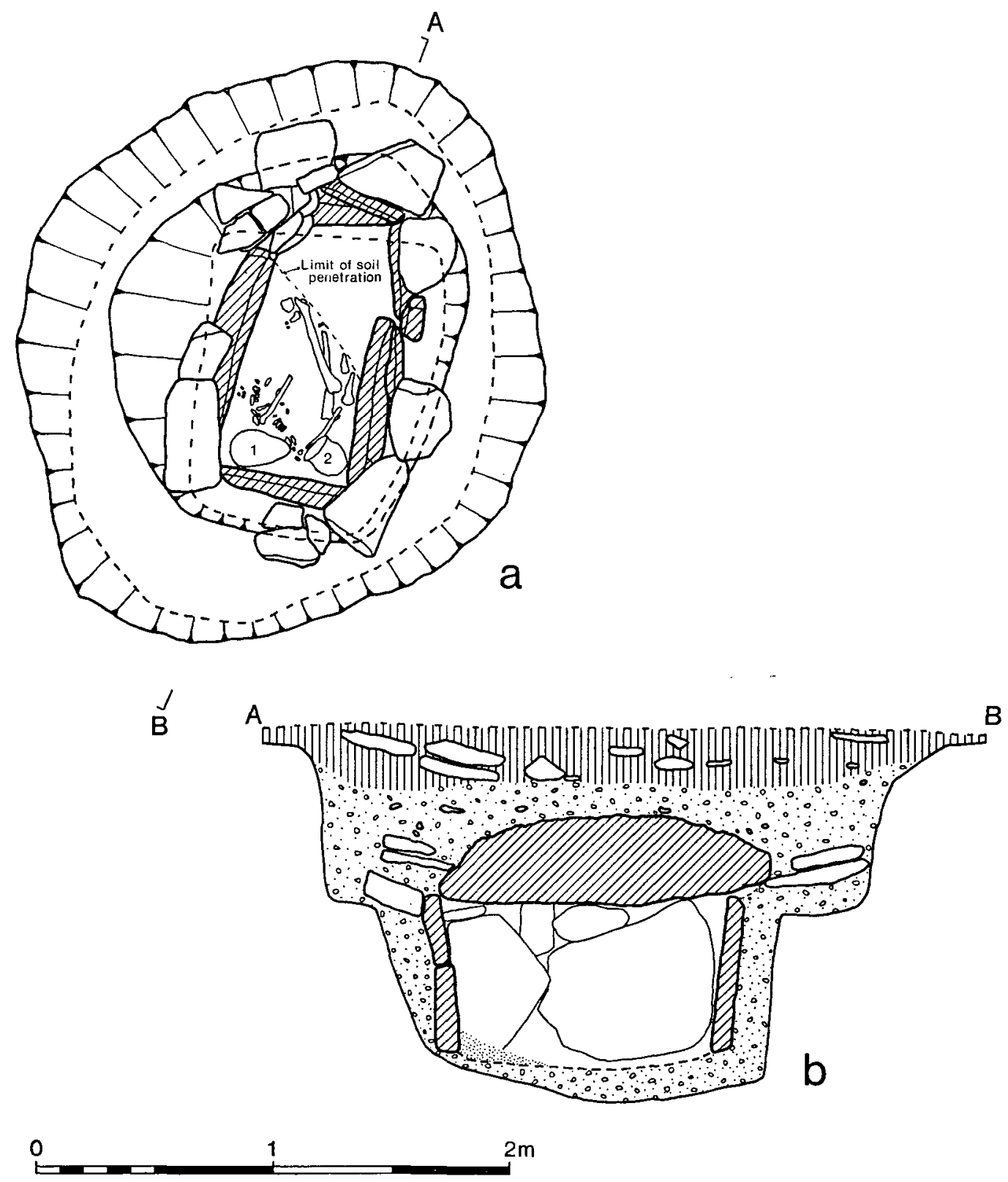

FIg 15 Henge Period III: Burial B: a - plan of burial (1 - skull, 2 - food vessel SF 17); b - section

the cover slab was laid. In the fill of the cist only tiny fragments of bone were found. No grave goods had survived. The modern ditch running across the site had cut the $\mathrm{N}$ edge of the pit (fig 4) to a depth of c $0.55 \mathrm{~m}$ but the disturbance did not extend to the cover slab or cist.

Burial D (fig 17a, b) (Cremation catalogue no 2; pottery p 157)

A roughly rectangular pit c $1.9 \mathrm{~m}$ long, $1.6 \mathrm{~m}$ wide at the surface and $0.55 \mathrm{~m}$ deep had been dug to 

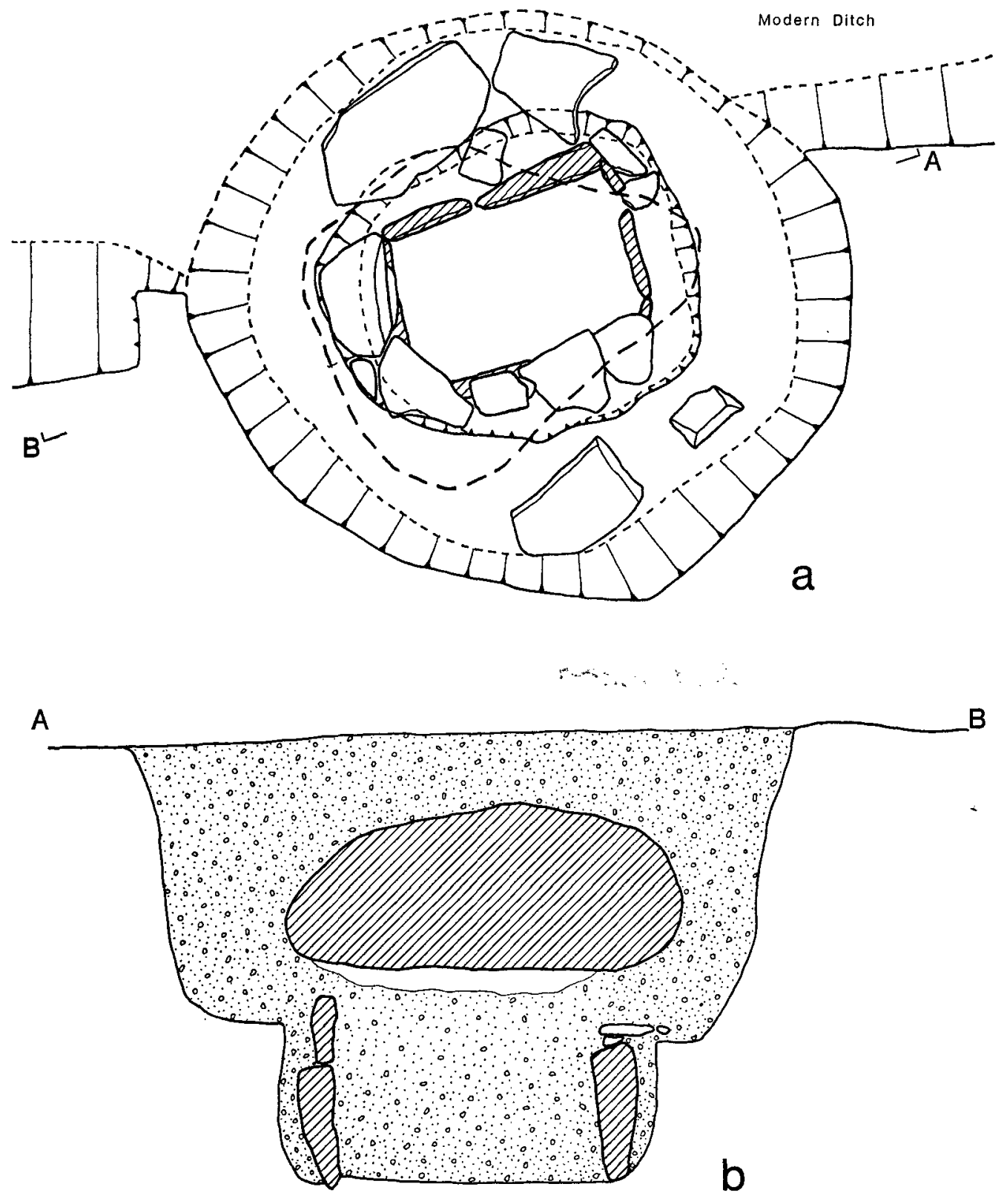

0 1 $2 m$

FIG 16 Henge Period III: Burial C: a - plan, position of cover slab indicated by heavy dashed line; $b$ - section 
receive a cist $\mathrm{c} 1 \mathrm{~m}$ long, $0.7-0.75 \mathrm{~m}$ wide and approximately $0.35 \mathrm{~m}$ deep. Relatively modern disturbances associated with the digging of long grave (LG7) and the breaking of a plough share in the cist had resulted in the removal of the SW end, most of the SE side and, presumably, the cover slab. The two surviving complete sides were constructed using a single slab for each, partly dug or pressed into the bottom of the pit. It is likely, however, that the SE side had been built of more than one slab. A vaguely defined dark stain (marked 1 on fig 17a) on the floor of the cist may have been the remnants of an inhumation. To the $\mathrm{N}$ of the stain lay a small deposit of burnt bone, possibly not human. Its neatly defined edges suggest that it may have been held in an organic container (? a bag) which has since disintegrated (marked 2 on fig 17a). A maggot-decorated food vessel (SF18), was found in the NW corner of the cist (marked 3 on fig 17). One part of the rim had been broken and dislodged from its position but otherwise the vessel was intact.

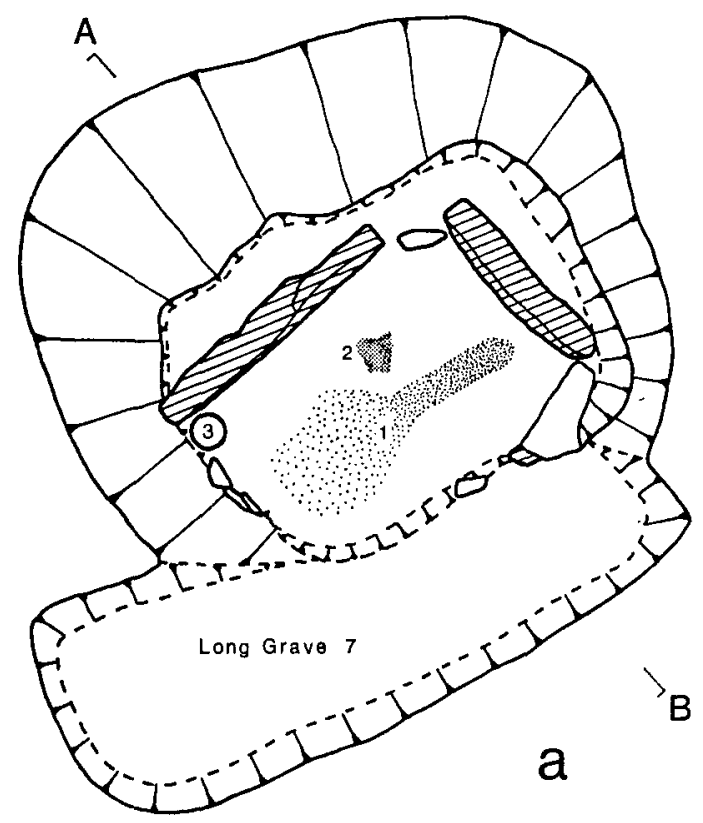

A

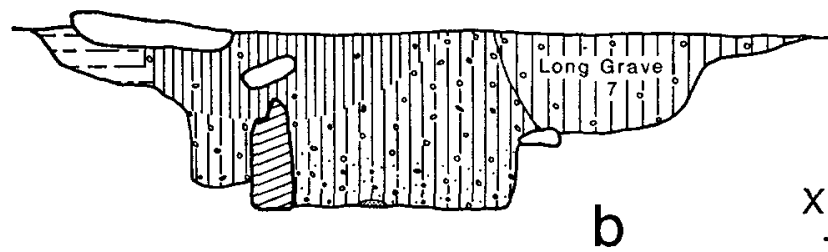

B
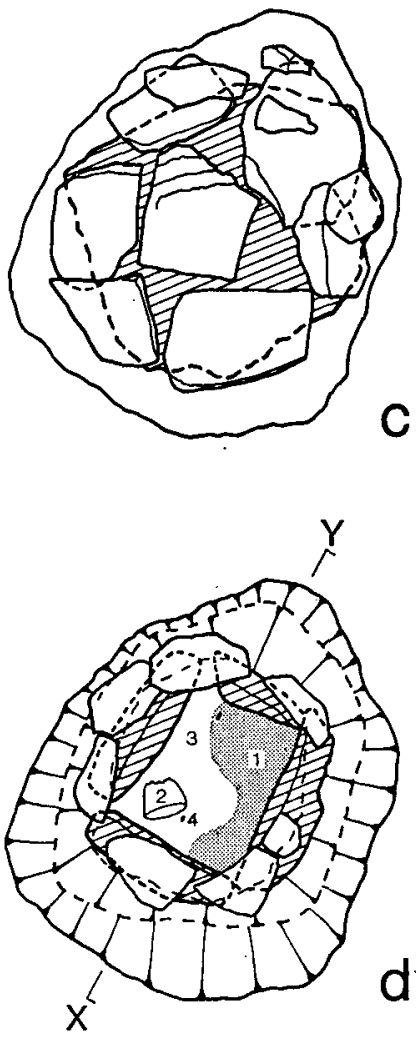

Burial F

Burial D

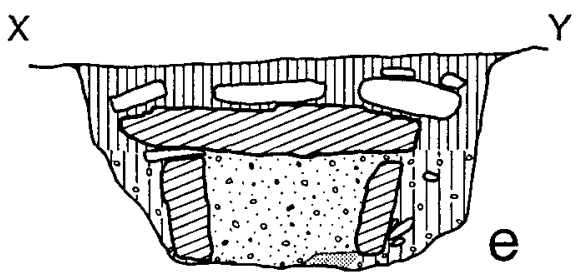

Fig 17 Henge Period III: Burial D: a - plan ( 1 - dark stain, 2 -cremation deposit, 3 - food vessel SF 18); $b$ section; Burial F: $c$ - plan, cover slab shown under additional slabbing; $d$-plan of burial (1 - cremation deposit, 2 - beaker SF 20, 3 - flint SF 43, 4 - flint SF 44); e - section 


\section{Burial E (fig 18) (Pottery p 157; Stone p 168)}

A vertically sided oval pit $1.75 \mathrm{~m}$ long, $1.3 \mathrm{~m}$ wide and $0.7 \mathrm{~m}$ deep had been dug through the inner edge of the henge bank on the $\mathrm{N}$ side of the enclosure. It contained tiny fragments of very soft unburnt bone concentrated at 1 on fig 18 (projected on section), sherds of a broken food vessel (SF19) and a naturally struck chert flake (SF41). The lower part of the fill was darker than the upper, perhaps as a result of staining by the decomposition of the body. The pottery was concentrated at 2 on the plan (position projected on section). A single sherd was found high in the pit (3 on fig 18).

\section{Burial F (fig 17) (Cremation catalogue no 3; Pottery p 157; Stone 168)}

An oval pit $1.3 \mathrm{~m}$ long, $1.1 \mathrm{~m}$ wide and $0.65 \mathrm{~m}$ deep had been dug to receive a cist $0.5 \mathrm{~m}$ long, $0.4 \mathrm{~m}$ wide and $0.35 \mathrm{~m}$ deep. The pit was backfilled around the cist to the top of the side slabs and flags were laid on top of the slabs apparently to provide an even platform for the cover. A number of flags were laid directly on top of the cover slab (fig 17, burial F, c) and the pit was completely backfilled. The cist contained the cremated remains of an adult male human concentrated in the $E$ half (marked 1 on fig 17d), accompanied by a beaker (SF20; marked 2 on fig 17d). Two burnt flakes (SF42; SF44) were found with the cremation deposit and an unburnt flake (SF43) was found beside the beaker. SF43 and SF44 (marked 4 and 3 respectively on fig $17 \mathrm{~d}$ ) are retouched pieces.

\section{Burial G (fig 19) (Cremation catalogue no 4)}

This oval pit was approximately $2.35 \mathrm{~m}$ long, $1.65 \mathrm{~m}$ wide and $1.9 \mathrm{~m}$ deep, stepped at $1.3-1.45 \mathrm{~m}$

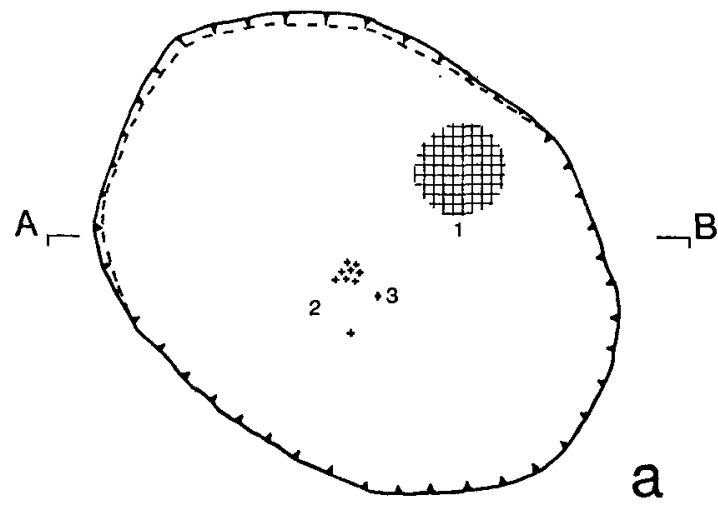

A
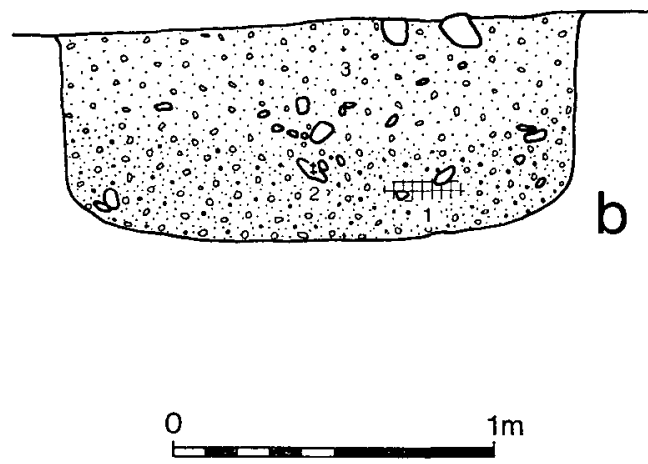

FIg 18 Henge Period III: Burial E: a - plan (1 - bone scatter, 2 and 3 -food vessel sherds SF 19); $b$-section 
below the subsoil surface. The unaccompanied cremated remains of an adult human male, probably under 35 years of age, covered the bottom of the pit, an area approximately 0.6 by $0.45 \mathrm{~m}$. Lumps of chalk were mixed with the cremated material as were two burnt bones, both possibly animal. The pit was backfilled with gravel and massive boulders.

Burials $\mathrm{H}$ and $\mathrm{J}(\mathrm{pl} \mathrm{10,11)}$ (Cremation catalogue nos 5 and 6; Pottery p 160; Stone p 168)

Two pits approximately $0.6 \mathrm{~m}$ in diameter and $0.45 \mathrm{~m}(\mathrm{H})$ and $0.3 \mathrm{~m}(\mathrm{~J})$ deep had been dug into the topmost fill of feature F51, which may itself have been a fourth example of the deep pit burials (fig 27; p 154). Each held an inverted urn $(H=S F 21 ; J=S F 22)$ containing cremated bone. Burial $H$, an adult
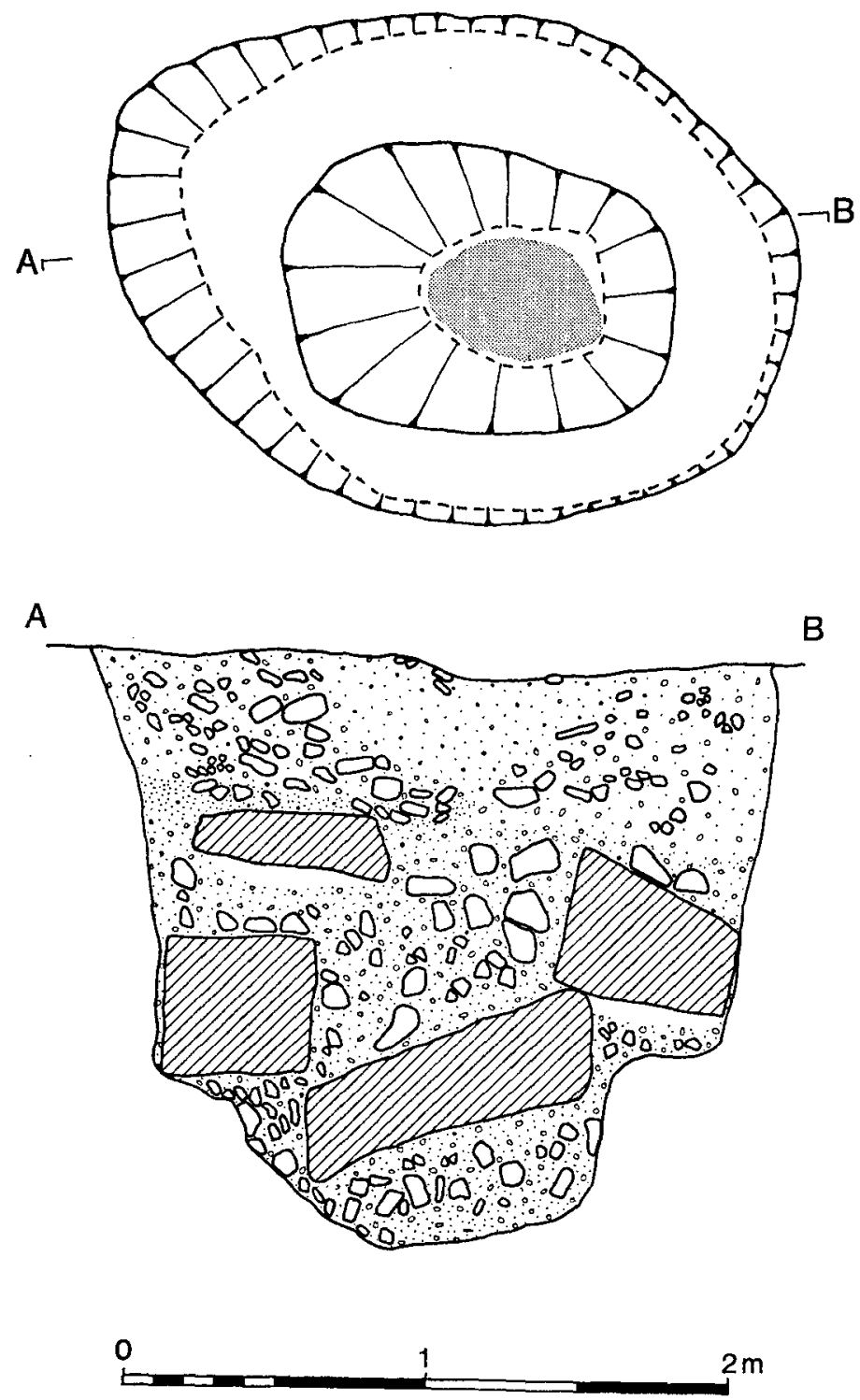

FIG 19 Henge Period III: Burial G 
human male (catalogue no 5), was unaccompanied. Burial $\mathbf{J}$ was a human adult male (catalogue no 6), possibly under 35 , and a child between four and six years old, accompanied by four burnt flints: two burnt white inner flakes (SF46; SF47) and a broken end scraper (SF48). Both urns rested on flat slabs. The urn of burial $\mathrm{H}$ was covered by a second slab.

\section{Burial K (pl 11) (Cremation catalogue no 7; Pottery p 161)}

A pit $0.55 \mathrm{~m}$ in diameter and $0.3 \mathrm{~m}$ deep had been dug near the $S$ terminal of the bank at the henge's W entrance. It contained an inverted enlarged food vessel urn (SF23) which held the unaccompanied cremated remains of a young adult human, perhaps female. The mouth of the urn did not rest on a flat slab as in $\mathbf{H}$ and $\mathbf{J}$ and the base of the urn had been damaged, possibly by recent ploughing. The close proximity of posthole F25, which may represent a marker of some sort, should be noted. burials.

In addition to burials B-K a pit (F51) found in the interior may have been a fourth of the deep pit

\section{PERIOD IV}

Four pits noted as dark marks on the aerial photographs and recorded as resistivity anomalies were found due $\mathrm{N}$ of the enclosure (F3, F4, F5 (fig 20), F6 (figs 21, 22)). Differential drying suggested the existence of a fifth pit between F4 and F5; it was not excavated. To the NE of the henge a similar anomaly was investigated but no feature was found. The four pits were substantially similar to each other in shape, size and alignment. The long axis of each lay N-S. The depths varied between 1 and $1.3 \mathrm{~m}$, the lengths between 2.5 and $3 \mathrm{~m}$. In two of the pits two phases of activity could be discerned; in the others (F4 and F6) three phases were noted. The first phase was the digging of the pits and their partial or complete backfilling, while the second, recorded in F4 and F6, was the cutting of a smaller pit into the backfill. The last activity in all of the pits, although preserved better in some than in others (see below), was the deposition of burnt bone in slight hollows left in the top of the fills, presumably as a result of their compaction and settling.

In F6, in the compaction-formed hollow, human cremated remains were found in a rough stone setting (burial $\mathrm{O}$ ) apparently having been drawn together there after cremation in situ. In F3 the evidence was more disturbed but the remains of a similar setting were noted, however, with bone of indeterminate species. Likewise F5 showed similar signs of the burning of indeterminate remains in the hollow. No signs of burning in situ were found in F4. No artefacts or dating materials were recovered from the primary phases of the pits, or the secondary recuts in F4 and F6. Nor was there any evidence for their function. However, the activity reflected in the last phases of F3, F5 and F6, the deposition of burnt material or in situ cremation, was relatively rich in both. Two dates are available for the burning in F6, (905bc \pm 85 (GU-1350); 1085bc \pm 70 (GU-1351), and one for $F 5$ ( $895 b c \pm 60$ (GU-1437)). Finds were restricted to a burnt quartz flake (SF49) from F3, two flint flakes from F4 (SF50; SF51) and two sherds from a single vessel in F6 (SF24).

\section{Feature descriptions}

F3 (fig 20) At the modern subsoil surface this pit measured approximately $2.5 \mathrm{~m}$ long and $1.9 \mathrm{~m}$ wide. It was $1 \mathrm{~m}$ deep and narrowed at the bottom to $2 \mathrm{~m}$ long and $1 \mathrm{~m}$ wide. It was filled to a depth of up to $0.35 \mathrm{~m}$ with a mixed layer of gravel, silt and sand (layer 1). This material was covered by a light loam (layer 2) which filled most of the remainder of the pit. At the surface a shallow depression had been filled with soil (layer 3) incorporating charcoal and burnt bone, possibly human (cremation catalogue no 28). Within the layer were the remnants of a stone setting similar to that in F6. A quartz flake (SF49) was recovered from the burnt fill (layer 3 ). The primary fills of the pit appeared to have been deliberately deposited. The surface on which layer 3 rested showed signs of burning. Burial P.

F4 (fig 20) This pit measured approximately 2.7 by $1.95 \mathrm{~m}$ at the surface, 1.9 by $1.2 \mathrm{~m}$ at the bottom 
144 | SOCIETY OF ANTIQUARIES OF SCOTLAND, 1983
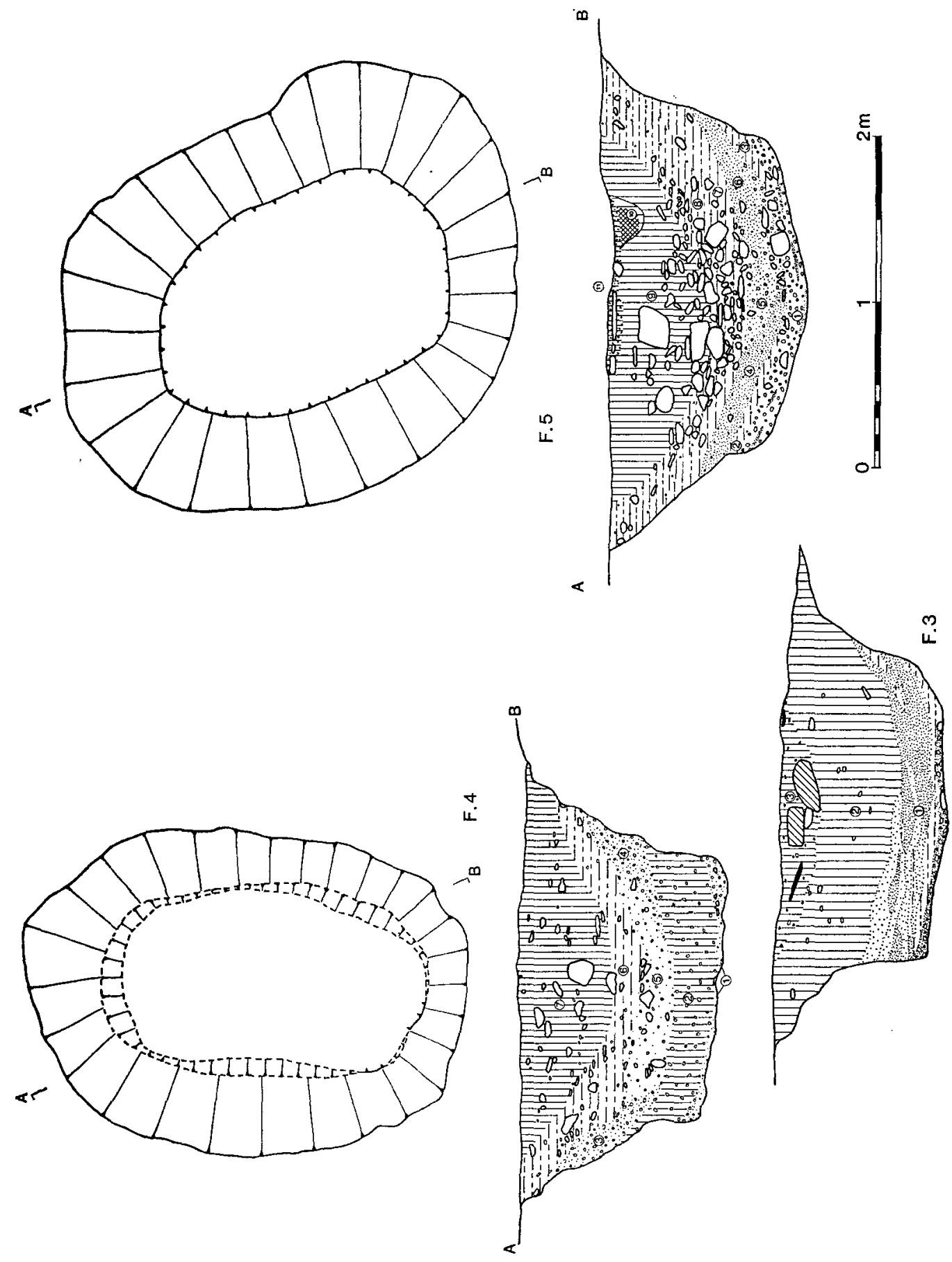

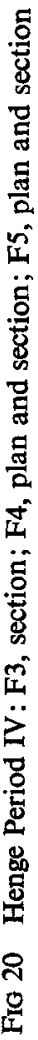


and a maximum of $1.25 \mathrm{~m}$ deep. A thin layer of sand and gravel (layer 1 ) covered most of the bottom, perhaps indicating that the pit had lain open for a time before it was backfilled. This layer was covered to a depth of about $0.25 \mathrm{~m}$ by a stony loam (layer 2). Above this the primary fills of the pit had been disturbed by a later cut. Undisturbed parts of the fills (layers 3 and 4) survived at the edges of the feature. The secondary cut was filled with two much stonier materials (layers 5 and 6 ) and a loam (layer 7). Isolated above the top of the pit as it survived were patches of charcoal and burnt bone of unidentifiable species (cremation catalogue no 29). Also within this severely disturbed layer were found two flint inner flakes (SF50; SF51). There was no conclusive proof of burning in situ.

F5 (fig 20) This pit was approximately $3 \mathrm{~m}$ long and $2.3 \mathrm{~m}$ broad at the surface, 1.9 by $1.25 \mathrm{~m}$ at the bottom, and $1.25 \mathrm{~m}$ deep. It was filled with gravel, sand and silt in the lower part (layers 1-6), and silt-loam and stones in the upper (layers 7-9). The stone element was concentrated near the centre of the pit, apparently as the result of gravitational sorting. Near the surface a small roughly circular pit had been dug from the bottom of the hollow formed in the top of the primary fills. It was backfilled with a heavily charcoal-stained soil containing fragments of burnt bone (layer 10; cremation catalogue no 30). The hollow contained a similar fill and its bottom showed signs of burning (layer 11). Burial Q.

F6 (fig 21, 22; pl 11) including burial $\mathbf{O}$ (cremation catalogue no 10). The pit measured approximately 2.65 by $1.7 \mathrm{~m}$ at the bottom and was $1.3 \mathrm{~m}$ deep. Because of misleading surface indications the longitudinal cross-section was not well placed to record the feature. The transverse section published here was not at $90^{\circ}$ to the longitudinal section. The first recorded event after the digging of the pit was its backfilling to a depth of approximately $0.9 \mathrm{~m}$ (layer 1 ). The pit was subsequently recut (layer 2; fig 22a) and filled with stones and gravel. Apart from two sherds in the primary fills (SF24) nothing was found in either the primary or secondary pits. The feature was backfilled to near the modern subsoil surface (layers 3 and 4). A hollow in the top of the pit, probably formed naturally by the compaction of the lower fills, was subsequently used for the cremation of a human adult (burial $O$; cremation catalogue no 10). The hollow was fire reddened and filled with charcoal (almost entirely of hazel) and cremated bone (layers 5 and 6). Most of the bone had been gathered together in a roughly rectangular stone setting (pl 11; fig 21, layer 8) constructed after the burning. The remains of an outer setting of stones (9) surrounded the feature. Any covering of the setting would have been destroyed by subsequent erosion and agricultural activity. Two charcoal samples were submitted for radiocarbon dating and produced the following assays: $905 \mathrm{bc} \pm$ 85 (GU-1350); 1085bc \pm 70 (GU-1351).

\section{PERIOD V}

Thirteen roughly rectangular pits varying in length from 1.8 to $2.5 \mathrm{~m}$ and in depth from 0.12 to $0.65 \mathrm{~m}$ were found in two groups in the $\mathrm{E}$ half of the henge (figs 4,23 ; table 3 ). The northern group was made up of five pits (LG1-5). In LG3 the extended skeleton of a female aged between 14 and 17 years was found ( $p$ 176); the head of the body lay at the $\mathrm{W}$ end of the grave. To the $S$ two straggling lines of similar pits (LG6-10; LG11-13) were excavated. Two (LG7; LG13) produced crumbs of bone. Fragments of burnt bone were found in three of the graves (LG2; LG7; LG10) but the extremely small quantities involved suggest that they were accidental inclusions. A smoothed stone slab (SF103) was found in LG1, at its W end. All 13 of the pits were aligned approximately E-W though some of the southern ones were nearer NE-SW. A further possible grave also aligned E-W may have been all but destroyed during the digging of the modern boundary ditch immediately to the $S$ of the $N$ group of graves.

Four of the pits had cut features of earlier periods. LG2 cut pit B/6, LG4 cut the pit of burial $G, L G 10$ cut the ramp of pit A/18, LG12 cut pit A/16, and LG7 cut the pit containing burial $\mathrm{D}$, causing considerable damage to the cist. Part of the skeleton found in LG3 was submitted for radiocarbon dating: $760 \mathrm{ad} \pm 60$ (GU-1382).

\section{MISCELLANEOUS BURIALS AND FEATURES}

Within and outwith the enclosed area a further 66 features, which could not be securely assigned to any of the foregoing periods, were investigated. Of these, 15 were deemed worthy of 


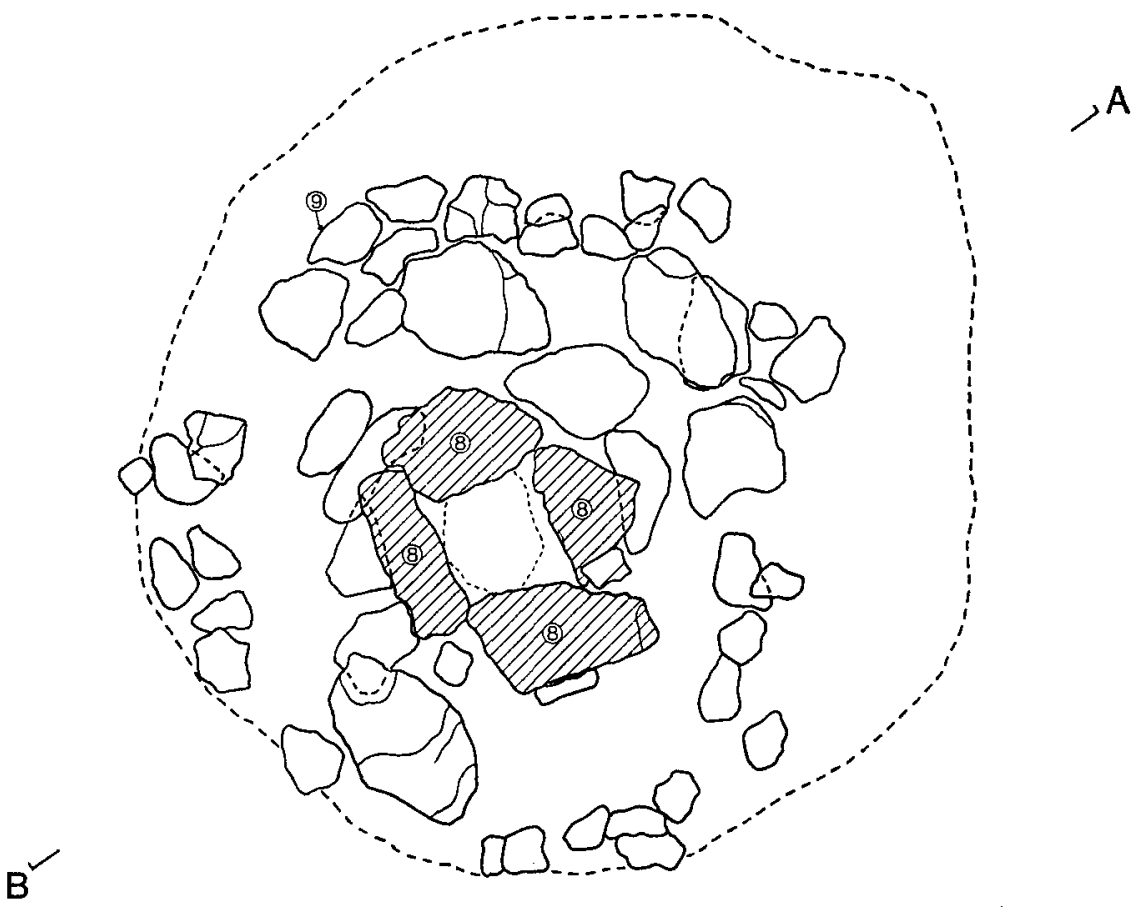

A
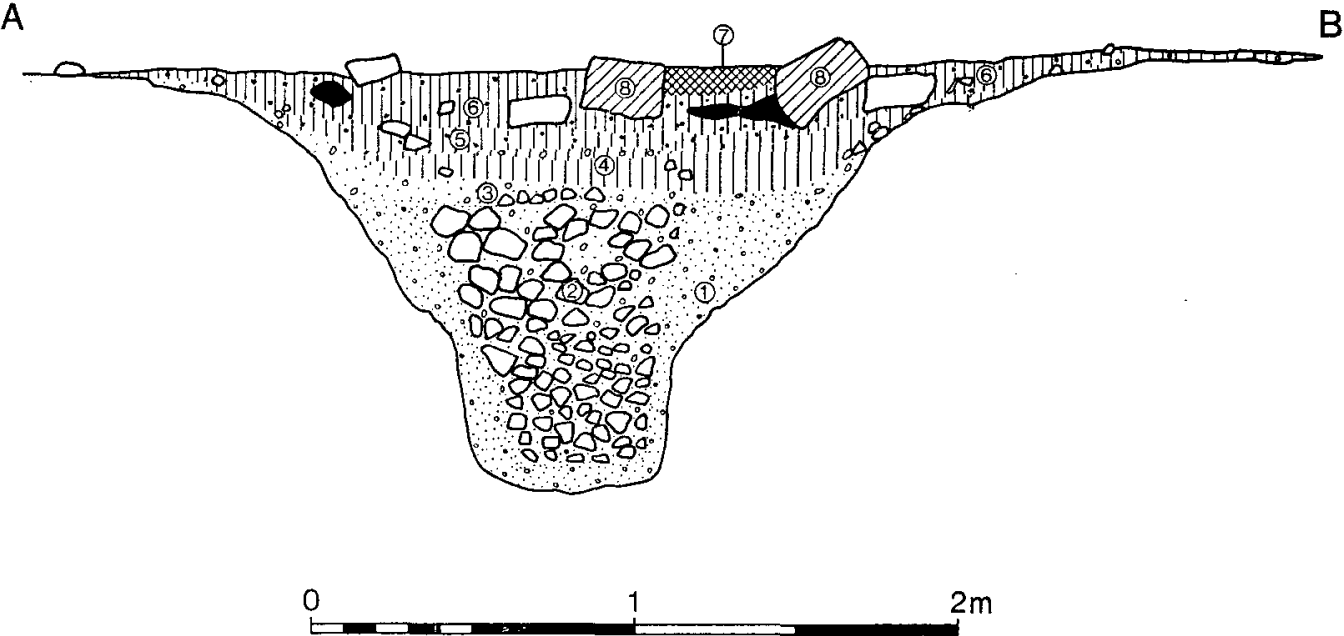

FIG 21 Henge Period IV : upper part of F6, Burial O

publication, in addition to timber ring B and three burials, the remainder contributing nothing to the understanding of the site as they produced no artefacts, and had no stratigraphic relationships or fills which provided a clue to their date and function. The 15 miscellaneous features published either have stratigraphic relationships, though not very useful ones, or have been provided by excavation with some clue to their functions. The presence of charcoal-stained soil in the fills of 

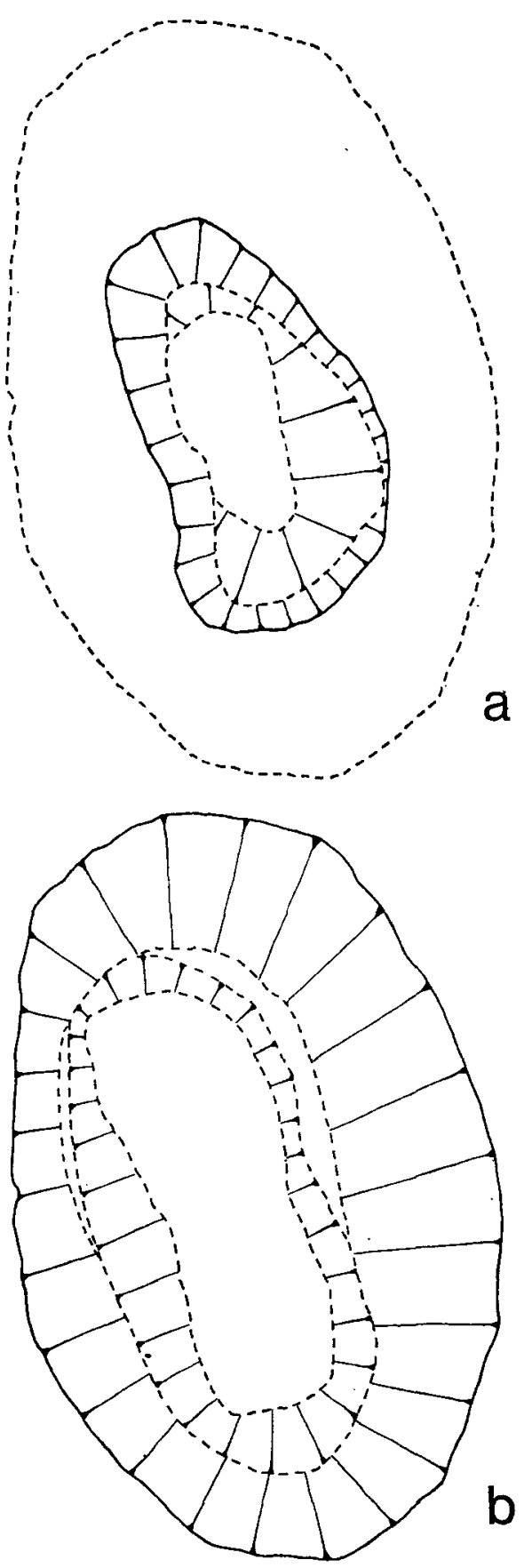

0 1

Fig 22 Henge Period IV: F6: a - after recut; b - empty 


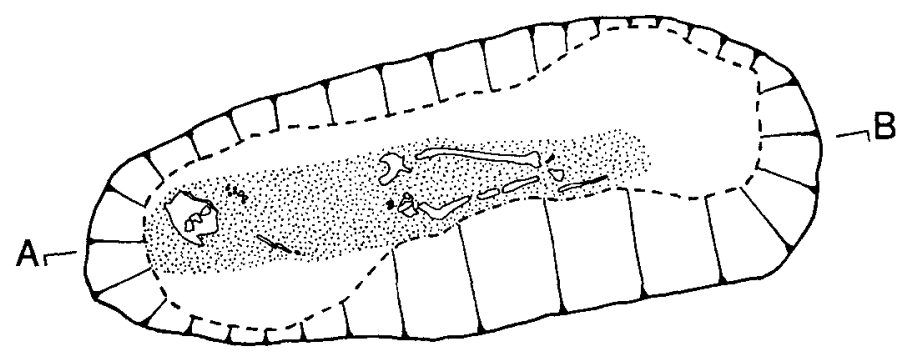

A

LG 3

B
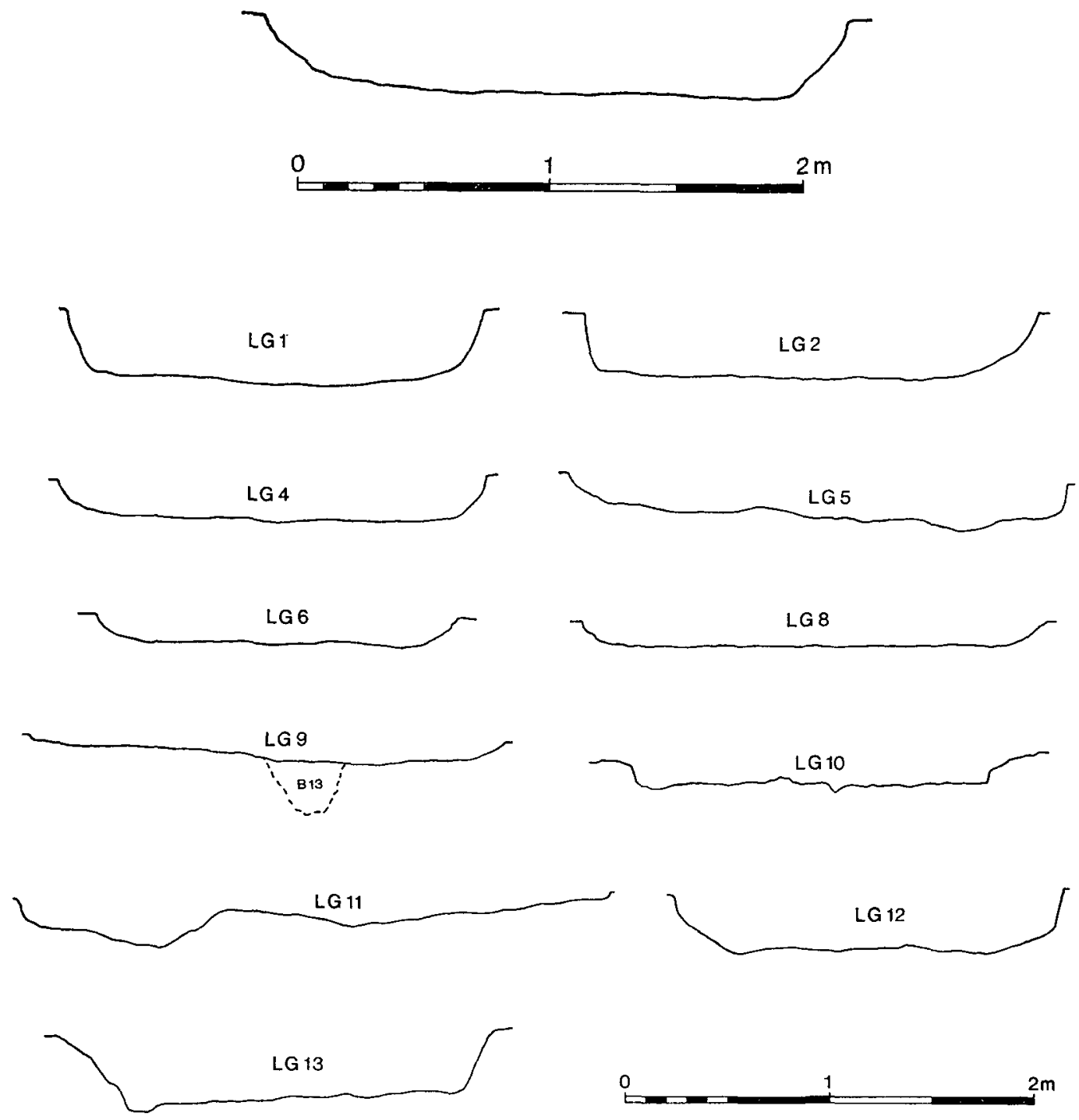

FIG 23 Henge Period V: Long Graves; shaded area represents stain 
TABLE 3

Dimensions of long graves

\begin{tabular}{|c|c|c|c|c|c|}
\hline Grave No & $\begin{array}{l}\text { Approximate } \\
\text { length } \\
\text { (m) }\end{array}$ & $\begin{array}{l}\text { Maximum } \\
\text { width } \\
\text { (m) }\end{array}$ & $\begin{array}{l}\text { Maximum } \\
\text { depth } \\
\text { (m) }\end{array}$ & $\begin{array}{l}\text { Azimuth } \\
\left({ }^{\circ} \mathrm{E}\right)\end{array}$ & Comments \\
\hline LG1 & $1 \cdot 95$ & 0.8 & 0.35 & $80 \cdot 5$ & $\begin{array}{l}\text { A smoothed slab (SF103) was found at the } \\
\text { W end of the pit }\end{array}$ \\
\hline LG2 & $2 \cdot 2$ & 0.8 & 0.34 & $89 \cdot 5$ & $\begin{array}{l}\text { Fragments of burnt bone (cremation cat no } \\
\text { 23) were found in the fill of the pit }\end{array}$ \\
\hline LG3 & $2 \cdot 3$ & 0.9 & $0 \cdot 3$ & $82 \cdot 5$ & $\begin{array}{l}\text { The skeleton of a female aged between } 14 \\
\text { and } 17 \text { years was found in the grave. The } \\
\text { head of the body was at the } W \text { end of the } \\
\text { pit (fig 23). }\end{array}$ \\
\hline $\begin{array}{l}\text { LG4 } \\
\text { LG5 } \\
\text { LG6 }\end{array}$ & $\begin{array}{l}2 \cdot 15 \\
2 \cdot 5 \\
1 \cdot 8\end{array}$ & $\begin{array}{l}0.85 \\
0.6 \\
0.45\end{array}$ & $\begin{array}{l}0 \cdot 25 \\
0 \cdot 7 \\
0 \cdot 7\end{array}$ & $\begin{array}{l}84 \cdot 5 \\
81 \cdot 5 \\
79 \cdot 5\end{array}$ & \\
\hline LG7 & 2 & 0.8 & 0.3 & $68 \cdot 5$ & $\begin{array}{l}\text { This grave cut prehistoric burial } D \text {. Frag- } \\
\text { ments of burnt and unburnt bone were } \\
\text { found }\end{array}$ \\
\hline LG8 & $2 \cdot 3$ & 0.8 & $0 \cdot 15$ & $63 \cdot 5$ & This grave cut F55 \\
\hline LG9 & $2 \cdot 4$ & 0.75 & 0.12 & $60 \cdot 5$ & This grave cut pit $B / 14$ \\
\hline LG10 & $1 \cdot 8$ & $0 \cdot 75$ & $0 \cdot 55$ & $83 \cdot 5$ & $\begin{array}{l}\text { Fragments of burnt bone (cremation cat no } \\
25 \text { ) were found in the fill }\end{array}$ \\
\hline LG11 & $2 \cdot 7$ & $1 \cdot 1$ & 0.25 & $91 \cdot 5$ & $\begin{array}{l}\text { This feature had suffered severe erosion } \\
\text { The average depth was below } 0.15 \mathrm{~m}\end{array}$ \\
\hline $\begin{array}{l}\text { LG12 } \\
\text { LG13 }\end{array}$ & $\begin{array}{l}2 \cdot 1 \\
2 \cdot 2\end{array}$ & $\begin{array}{l}0.8 \\
1.1\end{array}$ & $\begin{array}{l}0.65 \\
0.35\end{array}$ & $\begin{array}{l}89 \cdot 5 \\
86 \cdot 5\end{array}$ & \\
\hline
\end{tabular}

some might suggest contemporaneity with one of the phases of burning on the site (late Period II; Period III), but could of course relate to much more recent episodes.

\section{Burials}

Burials $\mathrm{L}, \mathrm{M}$ and $\mathrm{N}$ have been included in this section because they cannot with any certainty be assigned to a particular period. $L$ and $M$ resembled burial $A$ of Period $I$ in style but the wide occurrence of this sort of burial throughout the second millennium makes such simple comparisons misleading.

\section{Burial L (fig 24) (Cremation catalogue no 8)}

A pit $1.05 \mathrm{~m}$ long, $0.65 \mathrm{~m}$ wide and $0.15 \mathrm{~m}$ deep had been dug to the $\mathrm{SW}$ of burial $\mathrm{B}$. A small unaccompanied cremation burial, that of an adult human, perhaps female, had been heaped in the centre of the pit, which had then been backfilled. A carbonized hazelnut shell was found in the fill.

\section{Burial M (fig 24) (Cremation catalogue no 9)}

A pit approximately $1.1 \mathrm{~m}$ long, $0.8 \mathrm{~m}$ wide and $0.2 \mathrm{~m}$ deep had been dug between the bank and ditch. The relationship between the bank and the pit could not be established because of the erosion of the bank at that point. The fill of the pit had been badly disturbed by ploughing and animal burrowing but part of an apparently unaccompanied cremation burial, that of an adult, perhaps a female aged about 30 , was recovered. A single ovicaprid bone was recovered.

\section{Burial N (fig 24) (Cremation catalogue no 22)}

A pit, largely destroyed by the digging of the modern ditch across the site, survived to a depth of $0.2 \mathrm{~m}$, though its base lay $0.4 \mathrm{~m}$ below the subsoil surface. It measured 0.5 by $0.4 \mathrm{~m}$ at the base. It contained a uniform loam fill with $\mathrm{c} 30 \mathrm{~g}$ of burnt bone, identified as human or bovid. Mixed in with the bone was a burnt fragment of shell. D Heppell of the Department of Natural History, Royal Scottish Museum has contributed the following note:

The fragment is identified as a portion of the periostracum of Arctica islandica (Linnaeus 1767) to which the outer prismatic shell layer is still attached in places. The identification is based 


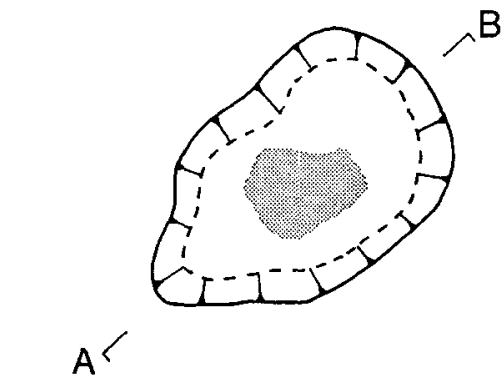

A

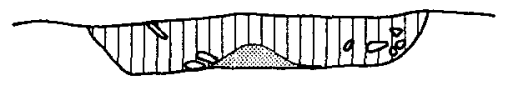

Burial L

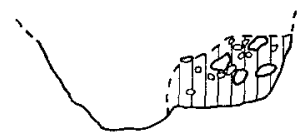

Burial N

B

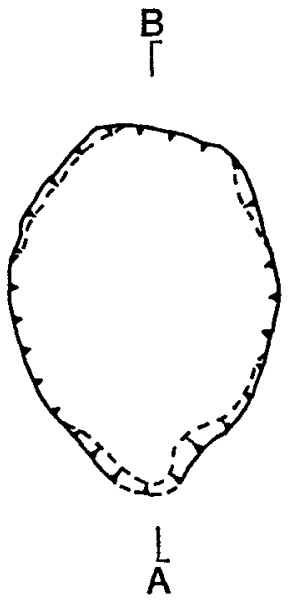

A

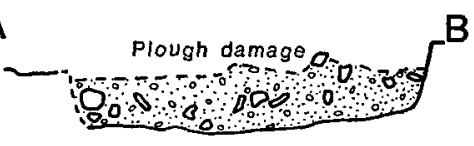

Burial M

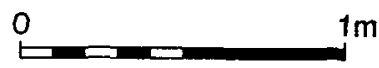

Fig 24 Henge miscellaneous features: Burials $L, M$ and $N$

on the thickness, dark colour, fibrous structure and size of the specimen. From its incremental lines it appears to be from the postero-ventral area.

Arctica islandica is an edible species, usually occurring sublittorally but occasionally found washed up. Dead shells are common on shore.

\section{Ring $B$ (figs 4, 25; table 4)}

Arranged in an ellipse approximately 22.5 by $18.5 \mathrm{~m}$ were 17 pits forming ring B. A possible eighteenth pit $(B / 18)$ was found on the surface near the $W$ entrance during the fifth trowelling of the area between pits $B / 1$ and $B / 17$ at the end of the third season, but insufficient time was available for its excavation. The setting of pits was not concentric with ring A or the bank and ditch (Period II), rather it lay very much to the $\mathrm{S}$ and $\mathrm{W}$ although still contained within the circumference of ring $\mathrm{A}$.

The excavation of the pits provided little evidence of their function. Six pits had been so severely damaged by later activity or erosion that little comment could be made on their fills $(B / 2 ; B / 3 ; B / 6 ; B / 10$; $\mathrm{B} / 13 ; \mathrm{B} / 14)$. Four showed fill patterns which might be interpreted as post-pipes or their remnants $(\mathrm{B} / 1$; $\mathrm{B} / 4 ; \mathrm{B} / 5 ; \mathrm{B} / 16$ ) but seven produced no evidence for their use, although it is conceivable that the fill patterns reflected the removal of posts $(\mathrm{B} / 7 ; \mathrm{B} / 8 ; \mathrm{B} / 9 ; \mathrm{B} / 11 ; \mathrm{B} / 12 ; \mathrm{B} / 15 ; \mathrm{B} / 17)$. No artefacts were found in any of the features nor was any charcoal recovered. Pit $\mathrm{B} / 5$ produced a very small quantity (under $5 \mathrm{~g}$ ) of burnt bone of unidentifiable species from its upper fill but it seems unlikely that such a small scatter was anything but an accidental inclusion, the result of root or animal intrusion. All the cross-sections of the excavated pits are illustrated in fig 25 . Table 4 summarizes the dimensions of the pits and their spacing (based on confirmed features). If the eighteenth pit (B/18) existed at the $\mathrm{W}$ entrance, between $\mathrm{B} / 1$ and $B / 17$, then the distance $B / 1$ to $B / 18$ would have been $3.7 \mathrm{~m}$ and from $B / 17$ to $B / 18,3.1 \mathrm{~m}$ rather than $6.8 \mathrm{~m}$ between $B / 1$ and $B / 17$. 
TABLE 4

Dimensions of postholes of timber ring B

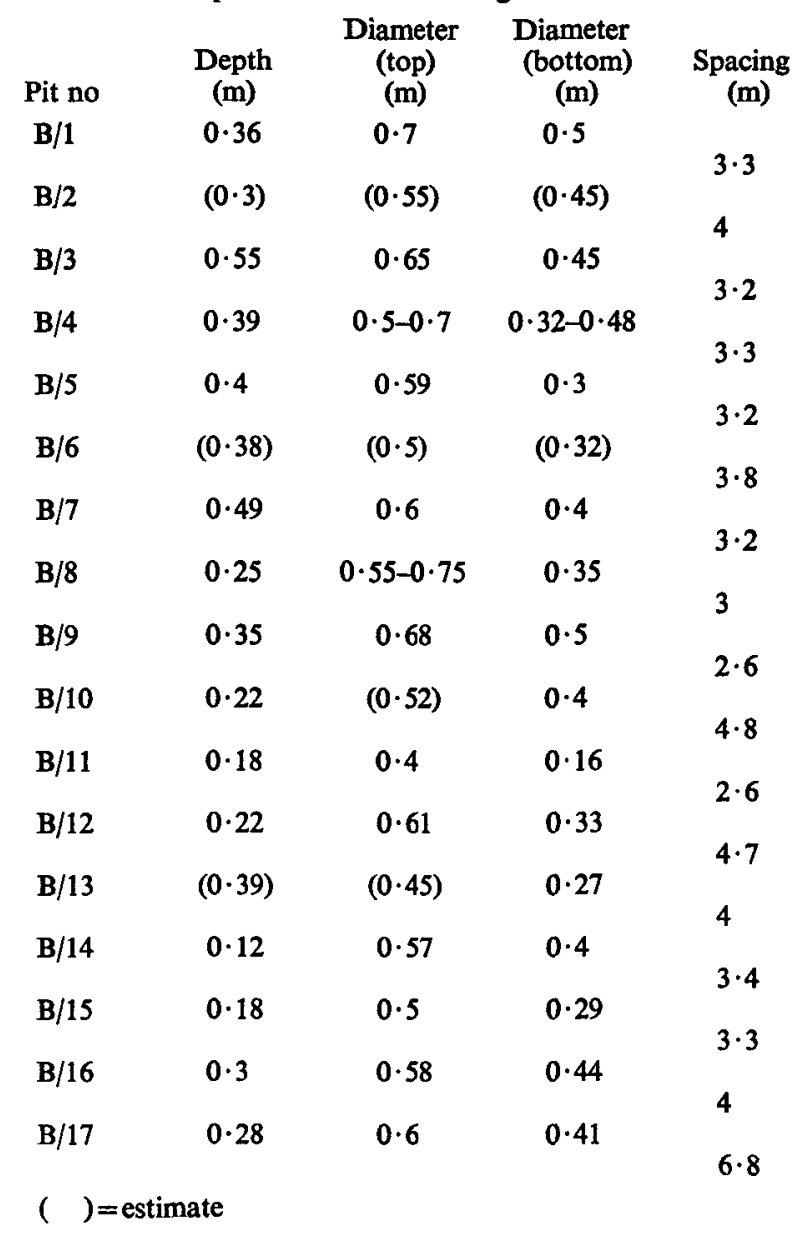

\section{Feature descriptions}

F2 (fig 26) This pit, cut through the Period II bank at the $\mathrm{N}$ side of the site, measured $3.25 \mathrm{~m}$ long, $1.4 \mathrm{~m}$ wide and $1.3 \mathrm{~m}$ deep. The fills were arranged in neat horizontal bands, suggesting some care in the filling of the pit. A massive boulder had been placed at the level of the modern surface. No dating material or artefacts were found. It is possible that the pit may have had a funerary function.

F23 (fig 26) This pit measured approximately 1.5 by 1.1 by $0.6 \mathrm{~m}$ deep. The silt fills appear to have been deliberately deposited. Charcoal flecks in the upper loam fill may indicate some relationship to the Period II or Period III burning episodes, though no burnt bone was found.

F25 (fig 26) This pit measured 0.3 by 0.35 by $0.3 \mathrm{~m}$ deep narrowing to $0.1 \mathrm{~m}$ at a depth $0.2 \mathrm{~m}$ below the surface. The lower gravel fill was covered by a loam. The pit may have been dug to hold a small post. The proximity of burial $\mathrm{K}$ should be noted.

F29 (fig 26) This irregularly shaped pit measured approximately 1.9 by $1.7 \mathrm{~m}$ and was up to $0.25 \mathrm{~m}$ deep. Within the loam fill were traces of charcoal, fragments of burnt bone (catalogue no 31 ) and sherds of pottery (SF27).

F37 (fig 26) This vertically sided pit was $0.35 \mathrm{~m}$ in diameter and a maximum of $0.4 \mathrm{~m}$ deep. The lowest fill, of fine gravel, covered the bottom to a depth of $0.12 \mathrm{~m}$ and extended up the sides. Above it was a loam containing some stones, covered in turn by a charcoal flecked loam. It is likely that this feature was a posthole, perhaps related to the late Period II burning. 


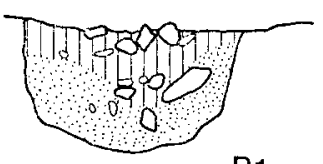

B1
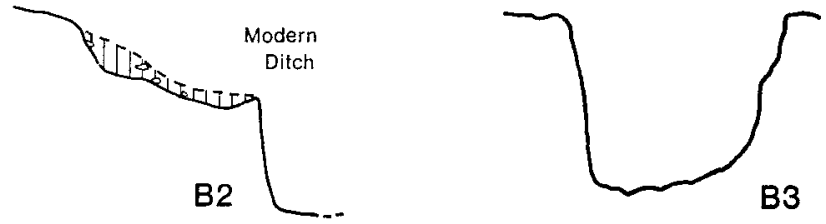

B3

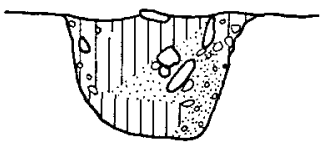

B4

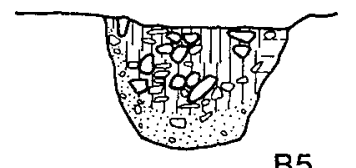

B5

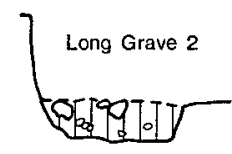

B6

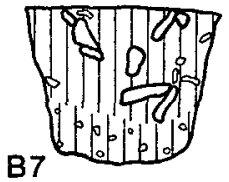

B7

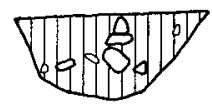

B8

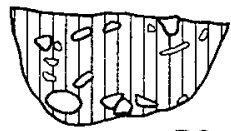

B9

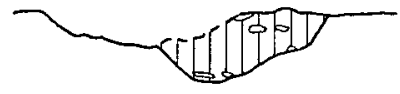

B10

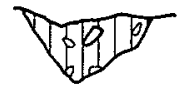

B11

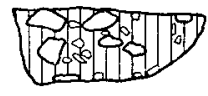

B12

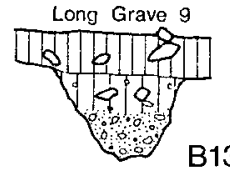

B13

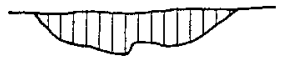

B14

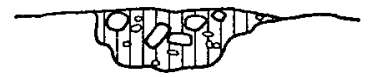

B15
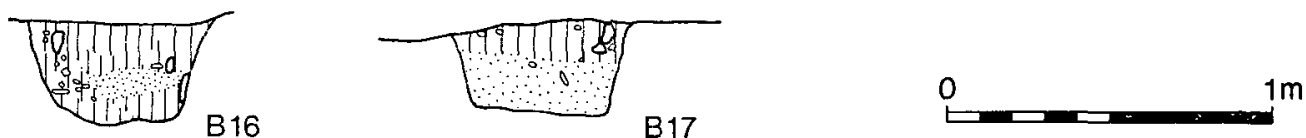

Fig 25 Henge miscellaneous features: Ring B, sections of all excavated pits

F38 (fig 26) This small pit, located $1.5 \mathrm{~m}$ to the $\mathrm{E}$ of $\mathrm{F} 37$ measured between 0.35 and $0.4 \mathrm{~m}$ in diameter and was a maximum of $0.28 \mathrm{~m}$ deep. The lowest fill, a fine gravel, covered the bottom to a depth of about $0.1 \mathrm{~m}$ and extended up the sides. Above this was a sand layer covered in turn by a loam. This feature may have been a posthole, perhaps forming a pair with F37.

F40 (fig 26) This feature, which had cut F39 (a small amorphous pit) measured between 0.8 and $0.9 \mathrm{~m}$ in diameter and was $0.4-0.5 \mathrm{~m}$ deep. Its lowest fill, a sand, completely covered the bottom. Above it to one side was a sand covered by silt, these two materials filling about one-third of the pit. The remainder was filled with gravel containing a high proportion of stones. Above it was a layer of charcoal-flecked and -stained soil. This feature may have been a posthole.

F41 (fig 26) This feature was approximately $0.9 \mathrm{~m}$ long and between 0.65 and $0.8 \mathrm{~m}$ wide. It was a maximum of $0.55 \mathrm{~m}$ deep and had steep sides. There were four fills. The lowest, sand, covered the bottom to a depth of up to $0.04 \mathrm{~m}$. Above it against the $\mathrm{N}$ side and standing half way across the pit was a layer of fine gravel. It was covered with two layers of loam which filled the pit. There was no evidence for the function of the feature. 

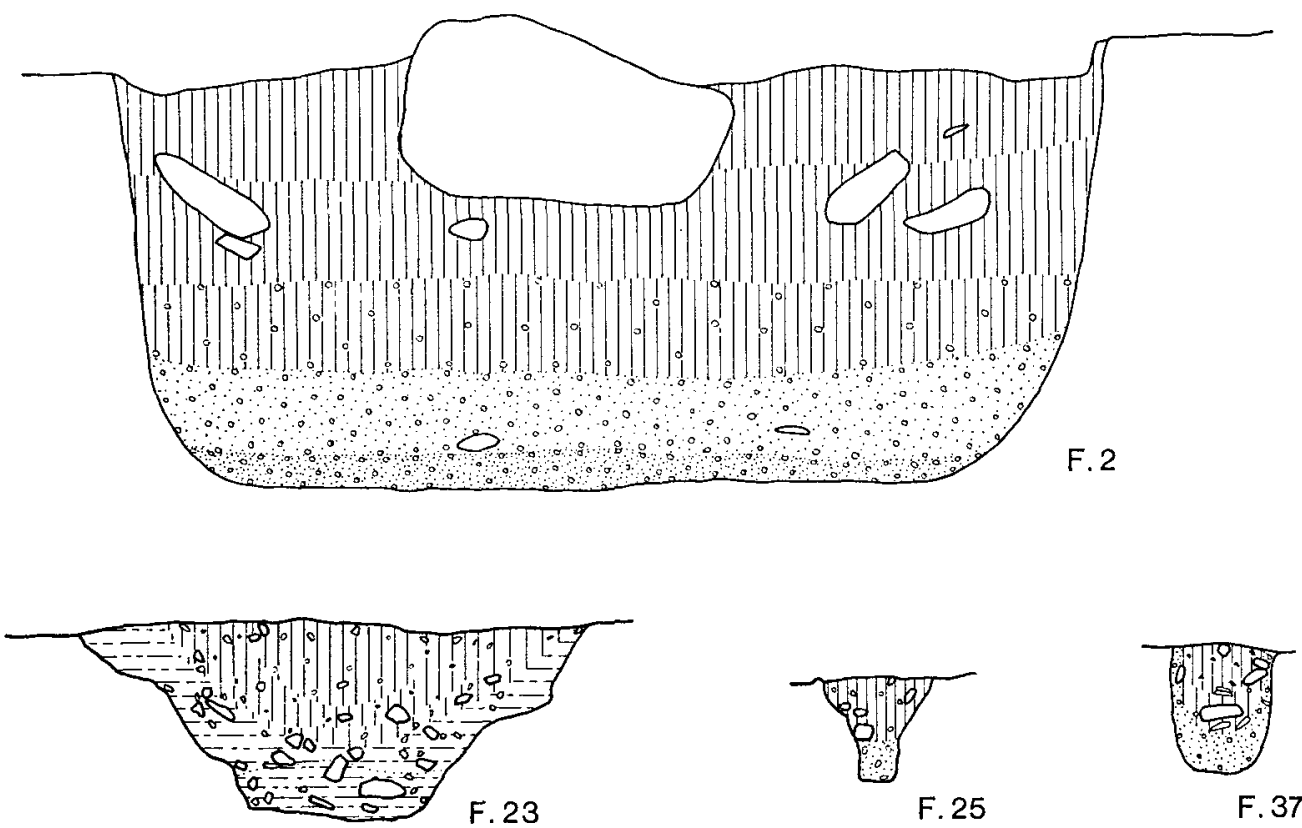

F. 25

F. 37

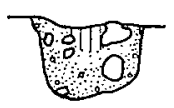

F. 38

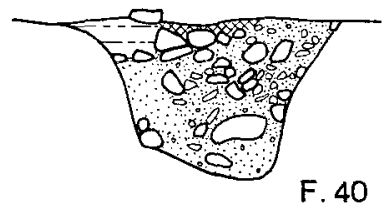

F. 40

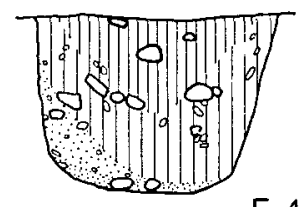

F. 41

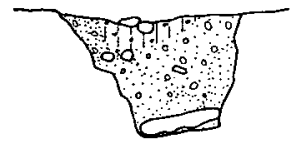

F. 42

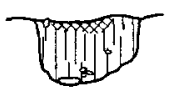

F. 43

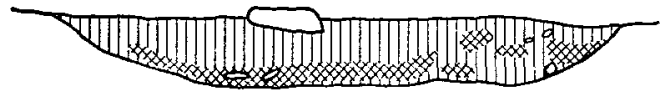

F. 29

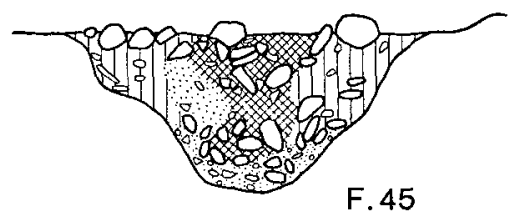

0 $1 \mathrm{~m}$

FIg 26 Henge miscellaneous: sections of pits 
F42 (fig 26) This pit measured between 0.6 and $0.65 \mathrm{~m}$ in diameter and a maximum of $0.39 \mathrm{~m}$ deep. A flat stone (SF104) lay at its base, covered by gravel which filled most of the feature. The gravels were vertically and laterally divided. At the top a small hollow was filled with charcoal flecked loam. It is likely that the feature was a posthole.

F43 (fig 26) This feature was between 0.3 and $0.38 \mathrm{~m}$ in diameter and a maximum of $0.19 \mathrm{~m}$ deep. It had three fills. The lowest was a light loam covering the bottom of the pit and extending up the sides, to the top on the NE. Above it was a darker loam, itself partly covered by a small layer of charcoalstained soil on the surface.

F45 (fig 26) The pit was $0.98 \mathrm{~m}$ long, $0.9 \mathrm{~m}$ wide and a maximum of $0.48 \mathrm{~m}$ deep. The lowest fill was a gravel covering the bottom of the pit. At the SE and NW there was packing of silt (with sand packing/slump also on the NW). Between the packing on either side was a vertical concentration of charcoal-stained soil (from the top of the lowest fill to the top of the feature). It seems.likely that this pit held a post whose pipe was preserved and filled with charcoal-stained soil and that this feature was associated with the burning at the end of Period II or during Period III. The charcoal was of willow.

F50 (fig 27) This pit had cut F51. It was $\mathrm{c} 1.9 \mathrm{~m}$ long, $1.4 \mathrm{~m}$ wide and was a maximum of $0.35 \mathrm{~m}$ deep. It was filled with gravel covered in the $S$ half by a charcoal-flecked loam. No charcoal pieces could be recovered. The charcoal may have originated in the Period III burning.

F51 (fig 27) This pit was $2.4 \mathrm{~m}$ long, $1.75 \mathrm{~m}$ wide and $1.2 \mathrm{~m}$ deep. It had cut an earlier pit (F44) and had been cut by another (F50). Two urned cremations had been deposited in pits cut in the top of the fill (burials $\mathrm{H}$ and $\mathrm{J}$ ). The pit had been deliberately backfilled. The bottom fill, c $0.4 \mathrm{~m}$ deep, was coarse gravel containing a high proportion of stones. Above this were lenses of gravel, sand and silt. A loam and charcoal-flecked loam filled a pit cut through these fills in the upper half of the feature. Charcoal was recovered from the flecked loam and the loam above it. No bone was found but it is possible that the pit once held an inhumation. The pit may be identified as a deep pit burial similar to burial $\mathrm{G}$.

F54 (fig 27) This pit had been cut by LG6. It was $0.4 \mathrm{~m}$ long, $0.23 \mathrm{~m}$ wide and would have been $0.37 \mathrm{~m}$ deep below the modern subsoil surface. It was filled uniformly with a stone-free loam.

F55 (fig 27) This feature had been cut by LG8. It was 0.8-1 $\mathrm{m}$ long (possibly overcut), about $0.8 \mathrm{~m}$ wide and a maximum of $0.3 \mathrm{~m}$ deep. The $S$ half of the bottom was covered with fine gravel. This was covered by a layer of loam containing few stones.

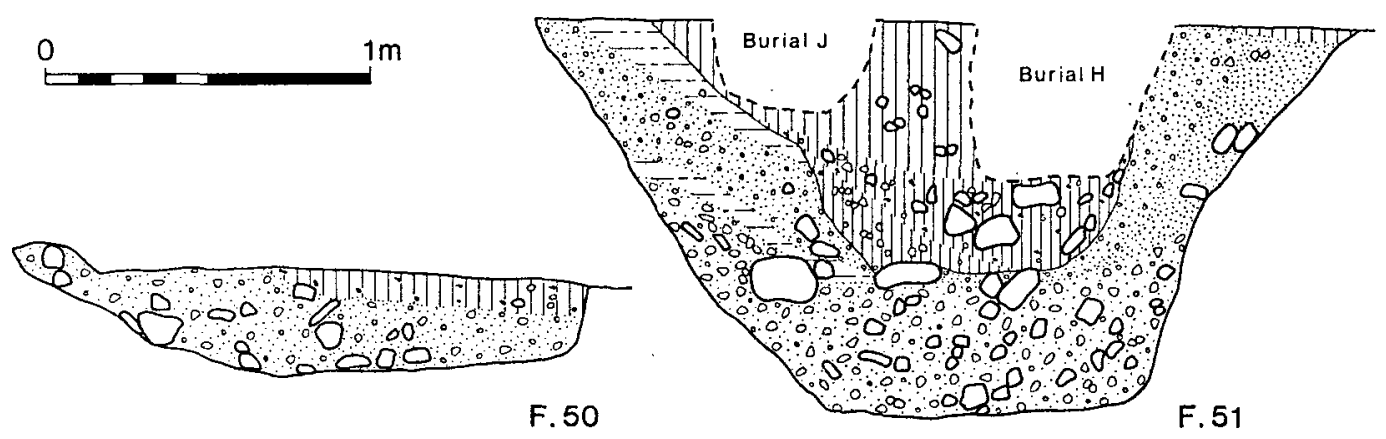

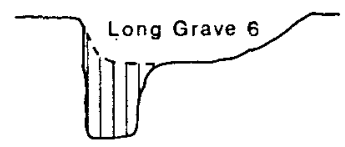

F. 54

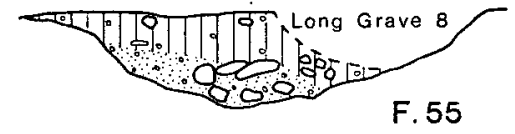

FIG 27 Henge miscellaneous: sections of pits 


\section{THE POTTERY FROM THE HENGE MONUMENT AT NORTH MAINS \\ $T$ G Cowie, National Museum of Antiquities of Scotland}

\section{INTRODUCTION}

The pottery from the henge consists of six substantially complete vessels recovered from funerary contexts (SF17, 18, 20-23), some 36 sherds, and over 50 fragments and crumbs many of which are featureless. Compared with the pottery from the mound, however, a greater proportion of the sherds and fragments can be interpreted as representing parts of the same vessels, and in one case, SF19, also from a funerary context, enough of the vessel is represented by the eight sherds and single fragment surviving to permit a reconstruction of the complete profile. Not including the seven more or less complete vessels thus associated with burials, the fragmentary material in the assemblage may represent parts of about 13 pots, but in the absence of rimsherds this must be taken as a minimum and cautious estimate only.

\section{CATALOGUE}

\section{Notes to the Catalogues}

1 Descriptions: only the most significant features of the assemblage have been recorded. The position and orientation of a piece is uncertain unless otherwise stated. Sherds are defined as having the internal and external surfaces surviving; fragments have only one of these surfaces surviving, and crumbs are arbitrarily $10 \mathrm{~mm}^{2}$ or less and retain no formal features.

2 The fabric groups are identified and discussed below on the basis of analysis by $\mathbf{M} \mathbf{J}$ Kenworthy p 257).

3 Relatively unabraded pieces are so noted.

4 Unless otherwise stated each catalogue entry represents a cautious separation into distinct vessels.

\section{Period I}

SF1 (fig 28) Rimsherd from vessel of uncertain form; the rim is almost rounded in profile but at the very top consists of a pinched, slighly angular junction; diameter uncertain; internal surface black to very dark grey, exterior very dark grey/black to dark brown; fabric $\mathrm{H}$. Also one body sherd, probably from near rim of same vessel.

SF2 Body sherd, fragment and three crumbs, possibly from the same vessel as SF1; possible fingertip impression at an angle on the external surface of the sherd, but rather abraded and unclear.

\section{Period II}

SF3 Three tiny body sherds and one fragment; fabric $\mathbf{H}$.

SF4 Body sherd; fabric J.

\section{Period III}

SF5 Six joining body sherds, one fragment and a crumb all from the same vessel; fabric $\mathrm{H}$; all relatively unabraded.

SF6 (fig 28) Six sherds, and fragments and crumbs in varying condition probably all from the same vessel; these include the two rimsherds illustrated, three body sherds, one base sherd and a fragment of the basal angle; form of vessel uncertain but rim appears to be of simple rounded type; two of the body sherds have slight shoulders on their external surface; diameter of vessel uncertain. Generally light or very pale brown to reddish yellow; fabric I. Burnt and abraded; surfaces very worn. Possibly parts of same vessel as SF9, 10.

SF7 Body sherd from vessel similar in fabric to, but thinner walled than, SF6; traces of incisions on external surface; burnt, abraded.

SF8 Body sherd from vessel similar in fabric to, but thinner walled than, SF6; bone-end impression on external surface; burnt, abraded.

SF9 (fig 28) Body sherd, possibly part of same vessel as SF6; generally very pale brown/buff colour, fabric I; decoration: on external surface, four incised parallel lines probably part of a filled triangle (cf SF10); burnt after fracture, very abraded and friable.

SF10 (fig 28) Three body sherds certainly all from the same vessel and possibly part of same vessel as SF6; position and orientation of sherds, and form of vessel uncertain (but see discussion below, p 162); internal surface and core reddish to light brown, core and exterior pale to very pale brown; 

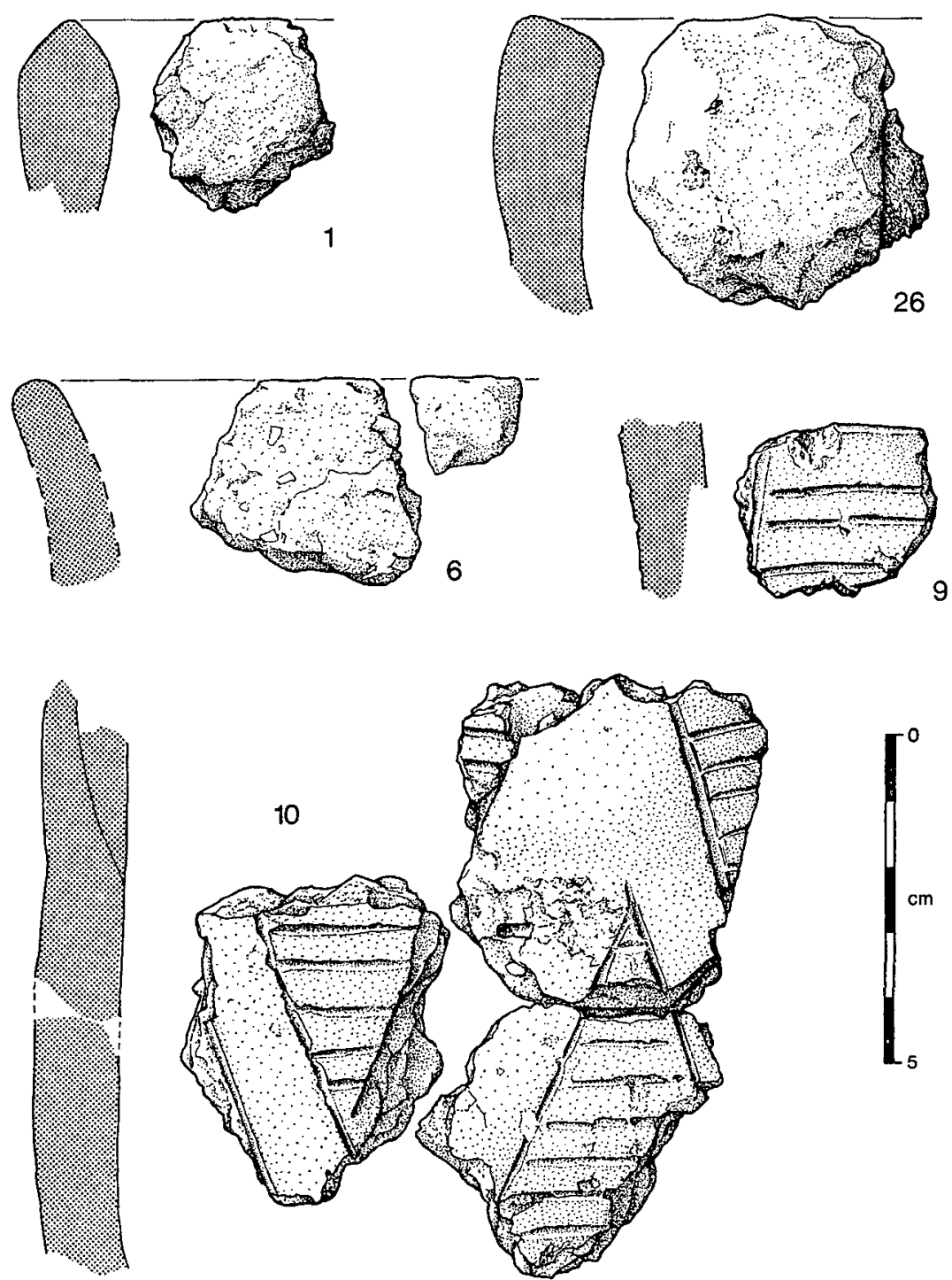

Fig 28 Henge pottery: miscellaneous sherds

fabric I; decoration: on external surface only; overall pattern unclear but the sherds contain parts of a design of filled triangles composed of incised lines and fairly deep slashes. The composition probably consisted of staggered, opposed, filled triangles, with horizontal incisions as the infill, and a reserved zigzag between the triangles; this decoration may have constituted the main zone of decoration on the body of a slightly waisted vessel of uncertain overall form - possibly a squat beaker, similar to but larger than SF20.

SF11 Fragment probably from a flattened base; possibly from same vessel as SF6, 9, 10; fabric I; possible bone or stick impression on external surface.

SF12 Fragment, possibly a very abraded base portion, (or part of a pottery disc); possibly part of same vessel as SF6, 9, 10; fabric $I$.

SF13 Body fragment, internal surface wanting; fabric I; partly burnt after fracture and abraded.

SF14 Body fragment, external surface wanting; fabric J. 
SF15 Body fragment, internal surface wanting; possibly from near base; fabric K; one light groove on external surface, possibly an accidentally incorporated plant impression; burnt and very abraded.

SF16 Twelve fragments and crumbs of baked clay (daub?); largest c 34 by $27 \mathrm{~mm}$; generally very pale or light brown; fabric L; burnt, soft and abraded.

SF17 Burial B (fig 29) Complete food vessel, wanting only two tiny fragments from the circumference of the rim; the vessel consists of a rather squat bipartite vase, with internally bevelled rim, the width of the bevel being $13 \mathrm{~mm}$. Owing to the way in which the lip of the rim has been moulded, the bevel is slightly 'stepped'. Externally the rim is slightly convex, giving way to a cylindrical neck expanding at the shoulder to a pronounced moulding c $50 \mathrm{~mm}$ from the rim. The convex lower body tapers quite sharply to an upright base, 7-8 mm high. Height $-125-32 \mathrm{~mm}$, rim diameter $-128-30 \mathrm{~mm}$, wall thickness $-10 \mathrm{~mm}$. Interior dark grey, with some lighter patches, exterior light brown; fabric $C$ (?). Decoration: on the internal bevel, jabbed impressions c $6 \mathrm{~mm}$ long have been applied transversely c $4 \mathrm{~mm}$ apart; a groove has been drawn with a spatula or similar tool around their outer edge to emphasize the rim and bevel, resulting in the 'stepped' effect already mentioned; on the exterior of the rim, vertical jabbed impressions c $5 \mathrm{~mm}$ long, at intervals of c $5 \mathrm{~mm}$ or less; on the neck, three lines of horizontal grooves, formed by dragging a spatula with a rather discontinuous action, and, below these, a row of opposed oval jabbed impressions forming a rough false-relief band, followed by a further three horizontal grooved lines; around the shoulder close-set jabs, c $3 \mathrm{~mm}$ apart; on the lower body, two grooved lines, a false-relief band, a further five grooved lines, another false-relief band, and a final two to three grooved lines extend the decoration to the foot, which is surrounded by close-set vertical jabs. The base is plain. Excellent condition - intact apart from some abrasion on one side.

SF18 Burial D (fig 29) Complete food vessel, undamaged apart from cracks in structure, and an abraded area on part of rim; the rim is simple with a narrow, fairly steep internal bevel, formed without the addition of clay; the exterior of the vessel swells to a widest point c $55 \mathrm{~mm}$ from the rim, but the overall shape is that of a squat bowl, with six moulded horizontal ridges around the circumference. The ridge around the shoulder and the one immediately above are c $23 \mathrm{~mm}$ apart, the remainder c $15 \mathrm{~mm}$ apart. Height $-119-22 \mathrm{~mm}$, rim diameter $-113-18 \mathrm{~mm}$, wall thickness 9-10 mm. Generally light brown and very dark grey; fabric I. Decoration: on internal bevel, transverse maggot impressions c $6 \mathrm{~mm}$ long at c $6 \mathrm{~mm}$ intervals; on the exterior, in each of the slight cavetto zones between the moulded ridges are vertical or slightly obliquely set maggot impressions, all $9 \mathrm{~mm}$ long, except in the broad zone above the shoulder where these are joined and up to $19 \mathrm{~mm}$ long. The base and the foot are undecorated.

SF19 Burial E (fig 30) Six sherds and three fragments representing parts of an incomplete food vessel; partly as a result of joins and partly as a result of overlaps in the ornamentation, the complete form and decorative scheme can be reconstructed with certainty. The vessel is a small bipartite vase; the rim is bevelled internally and owing to the way in which the pottery has flaked the method of construction is very clear: a 'false' bevelled rim has been capped by an applied strip of clay only just pressed over the edges - as a result the 'false' rim could well be mistaken for a rather abraded undecorated rim; the bevel is shallow but proportionately broad; below the well-defined shoulder, the lower body tapers to a substantial base c $20 \mathrm{~mm}$ thick. Height $-130-135 \mathrm{~mm}$, rim diameter uncertain, wall thickness - c $10 \mathrm{~mm}$. Internal surface light brown to light reddish brown, core very dark grey, exterior light reddish brown; fabric J. Decoration: on the internal bevel, four rows of $Z$-twist cord impressions, with a row of bone-end impressions c $4 \mathrm{~mm}$ apart on the inner edge; on the external edge of the rim, bone-end impressions, while the whole of the external surface is profusely decorated with triangular jabbed impressions, twisted cord impressions, and slashed lines. From the rim, the ornament is as follows: a single row of triangular jabs; a row of bone-end impressions; 6-7 rows of Z-twist cord, rather abraded in places; staggered, opposed triangular jabs forming a false-relief band around and emphasizing the shoulder; 9 horizontal rows of Z-twist cord, varying in the depth and firmness of the impressions; a further row of staggered jabbed impressions forming a false-relief band, haphazard in places; a zone of haphazard horizontal or oblique slashes and incisions arranged in about twelve lines; and, finally around the foot, another false-relief band composed of opposed triangular jabs. Around the edge of the base, there is a further row of jabs. Incomplete; individual sherds in relatively fresh condition.

SF20 Burial F (fig 30) Substantially complete beaker, wanting only parts of the lower body; the vessel has a simple, slightly inward sloping rim form, and a rather squat, shapeless profile, featureless 

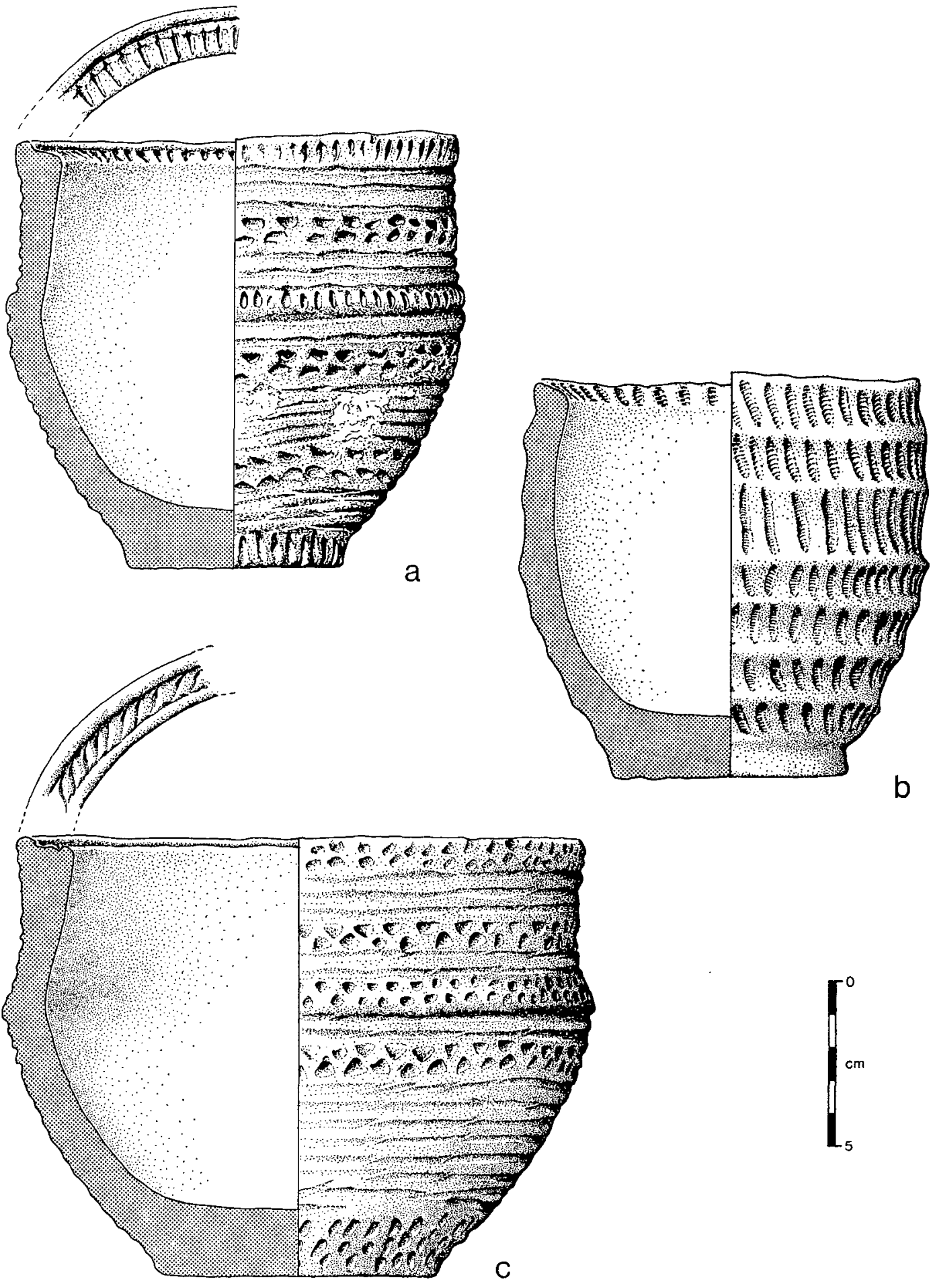

FIG 29 Henge pottery: food vessels, a - SF 17; b - SF 18; c - vessel from Cowdenhill, W Lothian (NMAS EE114) included for comparison with SF 17 

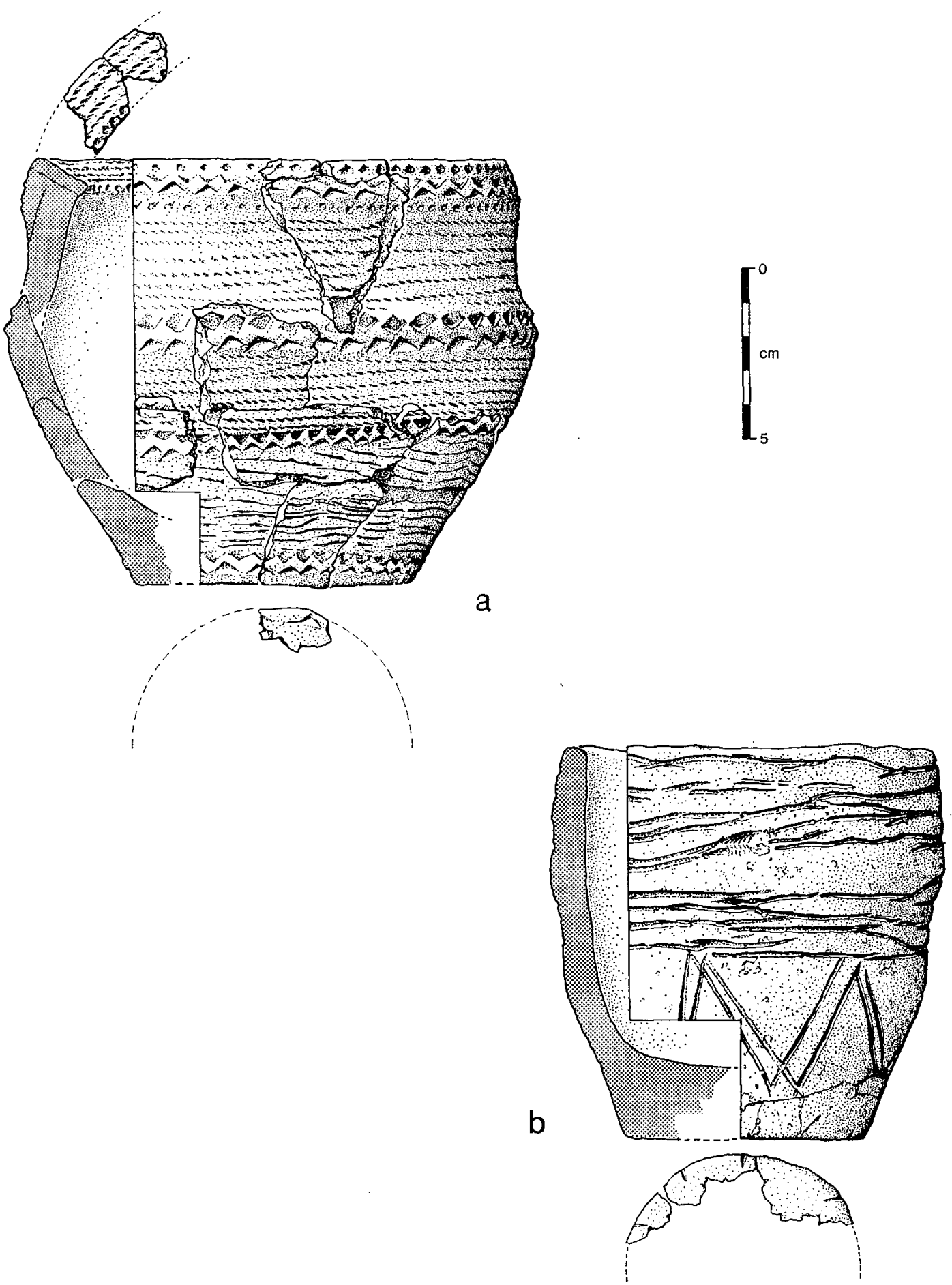

FIG 30 Henge pottery: a - food vessel SF 19; b - beaker SF 20 
apart from a slight waist, c $50 \mathrm{~mm}$ from the rim. Height $-114 \mathrm{~mm}$, rim diameter $-107-10 \mathrm{~mm}$, wall thickness $-8-9 \mathrm{~mm}$. Surfaces light brown, core very dark grey; fabric I. Decoration: on exterior, four or five roughly incised horizontal or oblique lines around the upper circumference, separated, by a gap of $\mathrm{c} 10 \mathrm{~mm}$, from a further four or five lines which fill the waist zone. Double incised lines form a zigzag or chevron around the lower body, stopping short of the foot. Around the edge of the base, there are some irregular jabs. The fabric of the base is very poorly fired, with a noticeably unconsolidated loose structure.

SF21 Burial H (fig 31) Large collared urn, restored from fragments; virtually complete apart from parts of lower body and much of base; on the interior, a deep concave moulding extends from the rounded rim to a depth of $\mathrm{c} 70 \mathrm{~mm}$; the collar of the urn is broad and deep expanding in diameter to $\mathrm{c} 320 \mathrm{~mm}$ at the junction with the neck; below the well-defined shoulder the lower body tapers to a proportionately small base; one small perforation is present on the collar, where it appears to have been made prior to firing. Height - c $330 \mathrm{~mm}$, rim diameter $-270-90 \mathrm{~mm}$, wall thickness c $15 \mathrm{~mm}$. Internal surface and core pale brown to dark grey, exterior light brown; fabric J. Decoration: on internal moulding, almost vertical to markedly oblique Z-twist cord impressions; exterior - around the collar, basic pattern consists of a running chevron composed of obliquely applied Ztwist cord lines; the triangles formed by these cord lines are bisected, approximately, by vertical cord lines, but in places the scheme is more irregular and incised lines, fingernail marks, and comb impressions also occur in rather random arrangements; on the neck, short cord impressions have been applied horizontally around the circumference in seven rather irregular rows; lower body plain. Thick encrustation obscured the interior of the collar prior to cleaning.

SF22 Burial J (fig 32) Bipartite urn, restored from fragments, largely complete but wanting parts of base and lower body; the upper part of the vessel presents a sinuous external profile: from the incurving rounded rim the body swells out and closes in again to a pronounced rounded shoulder, corresponding to a major oblique building line. The interior of the pot possesses a deep concave moulding

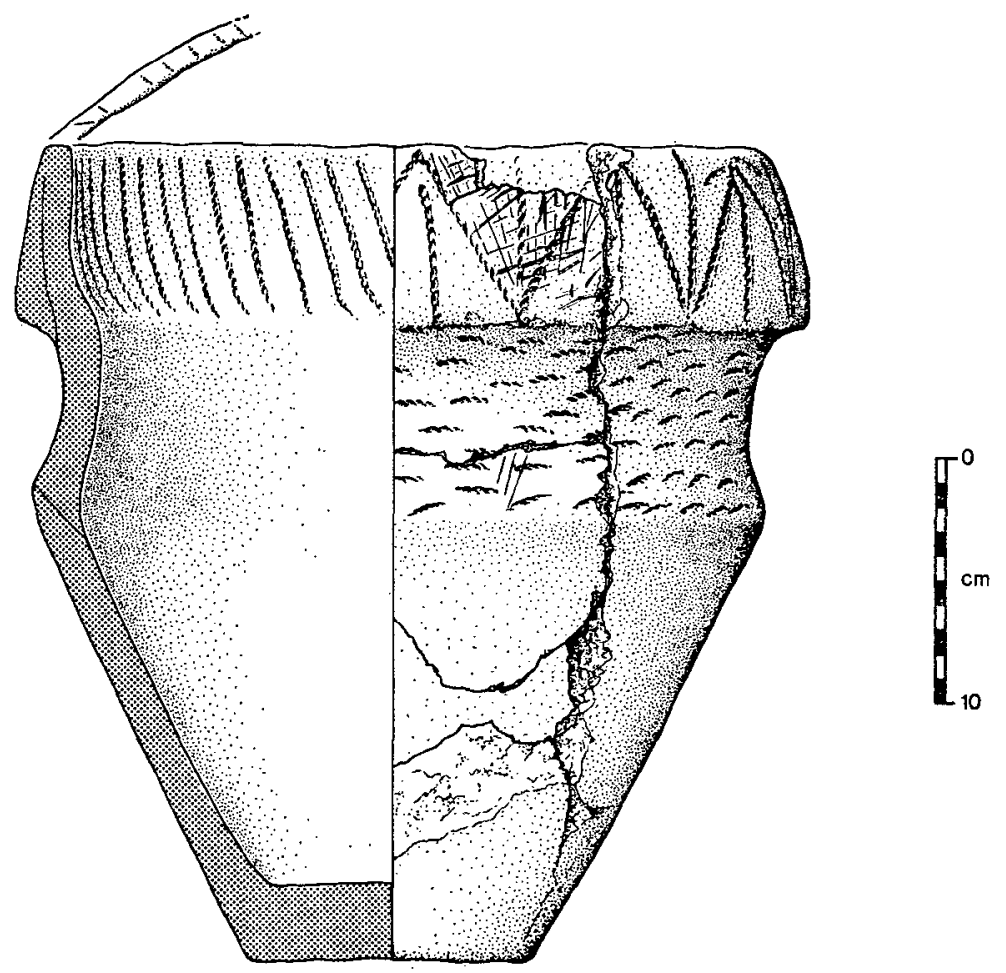

FIG 31 Henge pottery: collared urn SF 21 


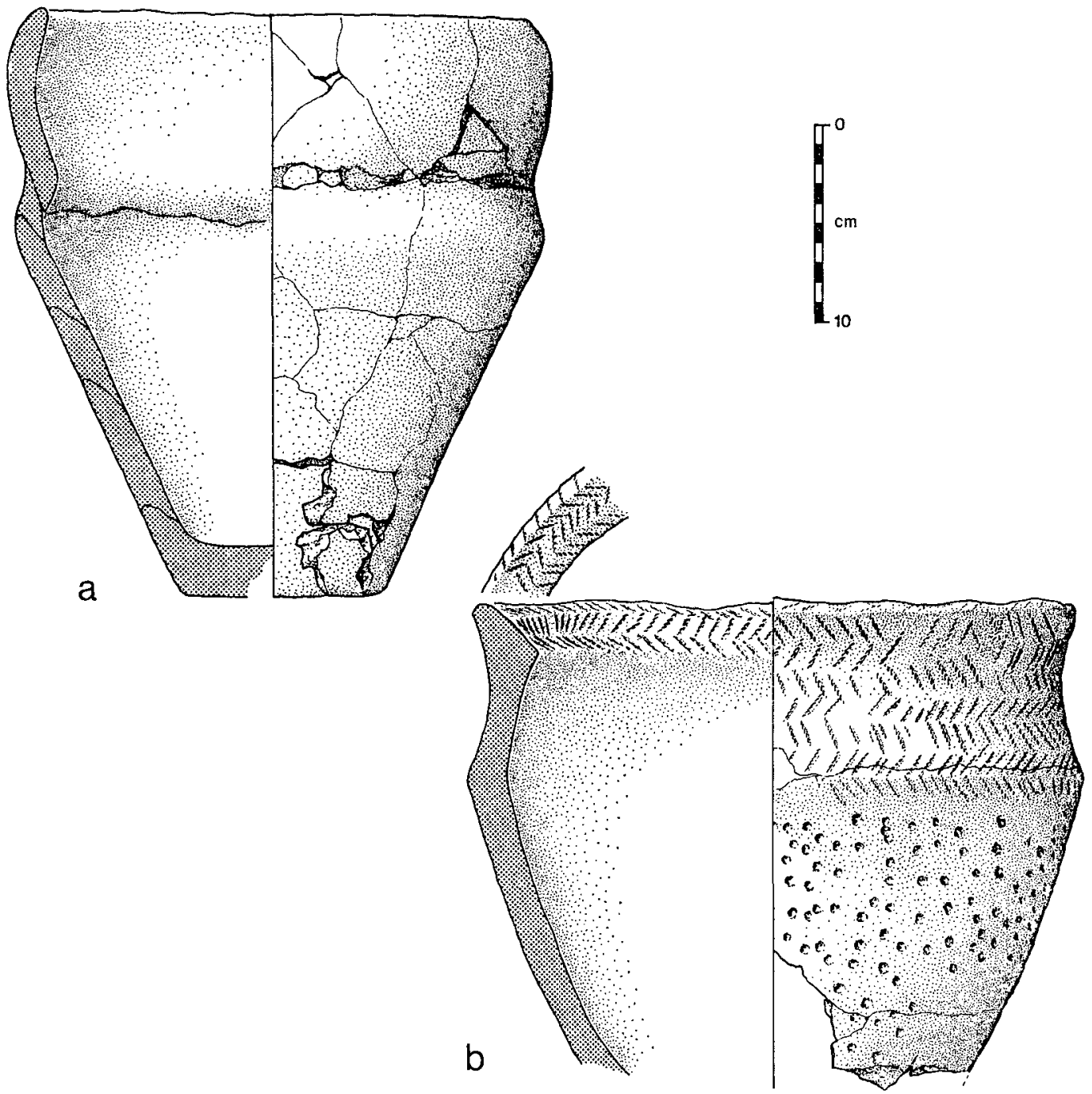

Fig 32 Henge pottery: a - bipartite urn SF 22; b-food vessel urn SF 23

extending to a depth of $\mathrm{c} 80 \mathrm{~mm}$ from the rim. From the shoulder, the vessel tapers to a proportionately narrow base. Height $-295 \mathrm{~mm}$, rim diameter - c $240 \mathrm{~mm}$, wall thickness $-13-15 \mathrm{~mm}$. Interior and core very dark grey; exterior light to very pale, pinkish brown; fabric $\mathrm{J}$.

SF23 Burial K (fig 32) Enlarged food vessel urn; restored; substantially complete apart from lower body and base; the rim has a broad internal bevel, c $35 \mathrm{~mm}$ wide with a slight external lip; the neck consists of a broad concave zone c $80 \mathrm{~mm}$ deep ending in a pronounced shoulder below which the surviving portion of the body tapers towards the now missing foot. Height (surviving) - c $250 \mathrm{~mm}$, rim diameter - c $300 \mathrm{~mm}$, wall thickness - 16-18 mm. Internal surfaces and core dark grey; exterior pale to light yellowish brown; fabric J. Decoration: on the internal bevel, short lengths of Z-twist cord arranged obliquely to form a chevron pattern; on the exterior, similar obliquely set short lengths of cord have been arranged in rows to form chevron patterns around the upper body; a further row of cord impressions has been applied around the circumference immediately below the shoulder, but the greater part of the extant lower body is decorated with an irregular all-over pattern of bone-end impressions. 


\title{
Period IV
}

SF24 Two body sherds probably from same vessel; fabric G; relatively unabraded.

\begin{abstract}
Miscellaneous
SF25 Featureless fragment and crumb; fabric $\mathrm{H}$.

SF26 (fig 28) Rimsherd and joining fragment; simple, rather flattened and slightly inturned rim from a vessel of uncertain form, but possibly a large bowl; rim diameter uncertain - c $200 \mathrm{~mm}$ ?; internal surface brown, core very dark grey; exterior reddish brown; fabric J; relatively unabraded. Also one body sherd possibly from same vessel.
\end{abstract}

SF27 Body sherd, possibly from shoulder area of vessel; fabric J.

\section{DISCUSSION}

Apart from the seven more or less complete vessels from funerary contexts, the pottery assemblage from the henge is differentiable largely on the basis of fabric rather than formal or decorative features. Much of the material can nonetheless be assigned, with varying degrees of confidence, to recognized ceramic traditions, and although these will be referred to in this discussion, the wider issues of the pottery assemblage and the relevant comparative material will be discussed below (p 248) in the light of the results of the excavations of all three North Mains sites.

\section{Form}

Most of the fragmentary material not found in association with burials consists of featureless body sherds or fragments and the position and orientation of these pieces on the vessels concerned is usually in doubt. The form of pot represented by the single rimsherd SF1 is uncertain although the wall thickness of $16 \mathrm{~mm}$ suggests a fairly sizeable vessel. The rimsherd, SF26, of simple, rather flattened type, presents the same problem: the wall thickness, $12-14 \mathrm{~mm}$, again suggests a substantial vessel - possibly a large bowl if the tentative Neolithic attribution on the basis of fabric is correct.

Over a dozen sherds and fragments, catalogued as SF6 and SF9-12, with shared characteristics in terms of fabric, general condition and context of discovery, appear to be elements of one or more vessels the shape of which must remain uncertain. However, on the basis of the two pieces of slightly everted rounded rim among the sherds SF6, the slight waisting of the body sherds SF10, and the hints of a flattened base, the group of sherds may cautiously be interpreted as being parts of a vessel (or vessels) similar to, though larger than the squat beaker SF20. This interpretation is reinforced by the distinctive decoration of the sherds SF9 and, more especially, SF10.

With one exception, the vessels recovered from the funerary contexts can be securely assigned to the traditionally accepted 'generic' categories of beaker (SF20), food vessel (SF17-19), collared urn (SF21) and enlarged food vessel urn (SF23). The remaining urn, SF22, is of an unusual form, lacking close local analogies while the total absence of decoration also tends to mark the vessel out as a relative rarity among northern urns, though the comparative material will be discussed in due course ( $\mathrm{p} 256$ ).

\section{Decoration}

Very little of the non-funerary pottery was decorated. In several instances, including SF2, SF11, and SF15, possible bone-end, fingertip or 'stick' impressions have been noted in the catalogue, but as such impressions provide no indication of the original design, if, indeed, any was intended, and may at times have been purely accidentally incorporated in the soft clay, they are not discussed further. As a result, the only significant group of decorated sherds is that represented by sherds SF7-11, some of which have already been referred to above as being possibly related pieces. SF7 and 8 are too fragmentary to merit more than a passing reference but the remaining sherds are of some interest. It is likely that the three sherds SF10 represent part of the slightly waisted body of a vessel cautiously interpreted above as a coarse beaker. On the basis of similarity of fabric, it is perhaps permissible to invoke the beaker SF20 as a possible analogue, and if the sherds SF6 and 9 are correctly interpreted as parts of the same vessel then a very tentative reconstruction of the decoration might be suggested as shown on fig 28 . SF20 is itself hardly a typical beaker, with its squat slack profile and carelessly applied incised decoration suggesting a late stage in the typological sequence.

With the exception of the plain bipartite urn, SF22, all the remaining funerary pottery recovered was ornamented using the techniques, if not the decorative patterns, commonly found in northern 
examples of the types in question. On the complete food vessel SF17, a simple but effective all-over arrangement of ornament has been produced by alternate use of grooves and jabbed impressions, possibly all made with the same implement - most likely some form of spatula with a rounded point. The incompletely represented vessel SF19 also bears profuse decoration incorporating cord impressions, incised or slashed lines, and bone-end and jabbed impressions, the last made with an angular point so as to produce false-relief designs around the exterior of the vessel. The remaining, ridged, food vessel SF18 bears a more straightforward repetitive pattern of whipped cord maggot impressions.

Twisted cord impressions provided the principal technique of decoration applied to the urns SF21 and 23 , although employed in quite contrasting ways. On the enlarged food vessel, short lengths of cord have been applied in quite characteristic fashion to create a series of chevron designs on the upper part of the urn, complemented below the shoulder by profuse all-over ornament made with the epiphsyis of a small bone. The principal motif on the collared urn consists of a corded zigzag applied in conjunction with vertical lines to form a series of bisected triangles around the collar, while short lengths of twisted cord fill the neck zone of the urn. In places on the collar, lightly incised lines suggest that an arrangement of the ornament in a series of filled triangles may originally have been in the potter's mind.

\section{Condition and context}

The pottery recovered from the henge amounts to some hundred pieces, ranging from indeterminate crumbs to virtually complete vessels. Three pots recovered from funerary contexts, SF17, SF18 and SF20 were clearly deposited as complete or near intact accessory vessels, and at least in the case of the food vessel SF17, pollen analysis (see p 178) has shown the pot probably contained a cereal-based liquid. In a fourth case, that of burial E, the recovery of only portions of a food vessel (SF19) from the fill of the grave might suggest a token deposit of pottery, or reinterment of parts of a broken vessel following disturbance of an earlier burial.

In respect of their burial inverted over cremated remains, the three cinerary urns (SF21-23) conformed to what appears to have been virtually an orthodox funerary practice. The perforation through the collar of the urn SF21 is worth noting. Its function is unclear but a repair or some connection with the attachment of an organic cover to the vessel are possibilities. Owing to breaks in the opposite quadrant it is impossible to be certain whether or not this was the only such perforation.

Of the more fragmentary material, the most significant group is that recovered from the upper fills of the large ramped postholes (Period III): the sherds SF6-11, interpreted above as parts of at least two vessels, most probably beaker(s), are distinguished by the similarities in their context and condition. The sherds and fragments show signs of scorching or burning and are noticeably soft and abraded, probably as a direct result of the effects of heating. From the same context as SF6 were recovered about a dozen pieces of baked clay (possibly used as daub?) but the interpretation of this material is uncertain. The presence of a small group of beaker sherds from a relatively localized area of the site might suggest the possibility at least of a disturbed burial or unspecified 'ritual' activity.

The material from the henge otherwise consists of an assortment of sherds lacking particularly diagnostic features, but interpreted on the basis of comparison of fabrics, and the known range of material from the Strathallan sites, as scattered Neolithic pottery. Such an interpretation might account for the rim SF26 and the body sherds SF3-5, 13-15, 24, 25, 27, and lastly but with less certainty the vessels represented by rim and body sherds SF1-2 recovered from the pre-enclosure old ground surface. The near total absence of those fabrics present in the barrow assemblage is noticeable and will be discussed presently ( $p 250$ ). The Neolithic pottery may best be interpreted as a residual scatter; hence its appearance in features of Periods I to IV

\section{THE INDUSTRY OF FLAKED STONE FROM THE HENGE MONUMENT AT NORTH MAINS}

C R Wickham-Jones, National Museum of Antiquities of Scotland, Artefact Research Unit

DESCRIPTION

Materials

The assemblage comprises 74 pieces, 53 of which are flint. Ten pieces of pitchstone and two of agate are also present. Quartz flakes recovered may be natural. 
There are only two pieces of agate. One is an unretouched inner flake, the other an unflaked pebble. The latter (SF52) is interesting as a prominent ring crack in the centre demonstrates the failure of an attempt to split it open for flaking. The assemblage is dominated by flint but the knappers were quite prepared to procure and work other stones if necessary. Pitchstone flakes in a similar fashion to flint although it may be more brittle. The discussion that follows refers mainly to flint as this forms the principal part of the industry. Fresh black nodular flint was not used at North Mains. The range of colours present and general condition of the pieces are characteristic of nodules that were collected as weathered pebbles. Pebble sources of flint do exist in Scotland (Wickham-Jones \& Collins 1978), most abundantly in the Flint Ridge in Buchan, Aberdeenshire (Gemmell \& Kesel 1979); it is likely that a more local supply, alluvial gravels for example, was exploited. Some of the flakes are quite large, indicating that a few of the pebbles at least were of a reasonable size. The flakes also suggest that the pebbles were of quite good quality flint, with few really bad flaws and little evidence of frost shattering. Flint is naturally black; the variety of colours present is derived from mineral action in the soil after it has been removed from its original context in chalk. At North Mains the pieces have corticated, and in some cases started patinating, after knapping (Shepherd 1972, 144-8).

\section{Technology: I primary knapping}

There is little evidence that knapping was carried out on site. A basic by-product in the reduction of nodules to flakes is the core. However, the assemblage contains only one core and no other indications of their presence such as core rejuvenation flakes or core trimming flakes. In addition to the ordinary flakes in the collection a number of pieces lack platforms and well-defined ventral surfaces but demonstrate a combination of other attributes involving general irregularities of shape and size. These pieces may be knapping waste (débitage). As such they would indicate a little on-site manufacture but some at least may be the result of other processes such as the breakage, resharpening and alteration of artefacts on site. However, some evidence may be obtained from the flakes in the assemblage about the techniques used. Fifteen preserve their platforms, indicating that an artificial platform was produced upon a nodule by the removal of a flake to provide a flat surface to allow the removal of further flakes. On only one of the flakes has a natural surface been used as a platform. Further preparation of platforms may be indicated by the removal of small flakes to form facets. This gives better seating for the hammer and strengthens the edge, making it easier to remove more regular flakes. The edge could also be strengthened and a more controlled blow obtained by trimming below the platform. Evidence of this may be seen on both the core and several of the flakes.

The nodules were probably hand held or supported on the knee during fiaking. There is no evidence of the use of the bipolar technique in which the core is rested on an anvil to be flaked either directly or through a punch. This method is particularly suited to the working of small pebble nodules as it increases the control of the knapper. At North Mains, however, the nodules were larger and the flint of good quality and the knappers were quite capable of producing a series of regular flakes without it.

Only a few flakes preserve enough detail for the analysis of the techniques and types of hammers used. There is no evidence for the use of hard stone hammers; both medium and soft were preferred and there is some suggestion that medium hammers were used in conjunction with the preparation, by facetting, of the platforms.

\section{Technology: II secondary knapping}

Retouching was used in two ways, to create a particular edge upon a flake and to shape flakes. There is no evidence of other methods of alteration and many of the pieces must have made quite suitable tools with no alteration at all. Five of the 10 retouched pieces have been burnt and damaged but where it is possible to examine it closely the retouch is very irregular. The scars vary greatly in size and shape even on a single piece. There are no examples of regular, for example parallel, flaking. SF76, from within the henge, has a small area of long steep retouch at the distal end but it also has areas of small, wide retouch and on both this and the other pieces the scars are largely determined by the morphology of the individual flake. SF43 for example, also from within the henge, has only tiny nibbling retouch which has served to remove the cortex from the edge of the flake. Secondary work was mainly carried out upon inner flakes but this reflects their numerically greater presence within the assemblage as a whole and it is perhaps more surprising to find that two of the retouched pieces are upon secondary flakes and a further two upon primary flakes. No two of the retouched pieces are the same and there does not seem to have been any 
selection of blanks for retouch upon any basis other than their spontaneous suitability for a particular purpose.

\section{The morphology of the retouched pieces}

The surviving pieces in the assemblage do not represent consistently recurring shapes, nor, with four exceptions (three of which, SF61, SF33, and SF48, are very general), are they morphologically duplicated upon other sites. Two pieces, SF38 and SF44, have been so damaged by burning that no trace of their original morphology remains. Three, SF72, SF43 and SF62, bear only small areas of retouch. Each has a large amount of cortex, two are, in fact, primary flakes; all are good examples of the rapid use of a small amount of retouch to form a particular working edge. Five flakes, however, demonstrate the more complex use of retouching work to shape the piece as well as to create an edge. Two of these from F33, SF39 and SF40, join to form a single artefact and have been considered as such. The four morphological types concerned do exist on other sites but give only general, uninformative parallels.

SF48 was found, together with two burnt unflaked flints, in the urn associated with burial J (Period III). It has been burnt and is badly damaged but enough remains to ascertain the general shape. A number of names might traditionally be ascribed to this piece of which 'end scraper' or 'knife' would be most usual though the shape occurs in a wide variety of contexts and is potentially useful for a large number of tasks. All parallels are therefore very general and of morphology only.

This latter point is true for two of the other pieces. SF33, incorporated in the Period II bank, is a bilaterally edge-retouched flake. Although it is broken, only the distal tip is missing: the two straight sides, both irregularly retouched, converge from a broad proximal end towards this distal. The piece might traditionally be called a 'knife' but once again this is in the absence of functional analysis. SF61 was found within the henge. Irregular retouch has been used all around the flake to create a roughly circular plan and traditionally it would be called a 'discoidal scraper'. The final retouched piece, a widely recognizable morphological type, is the barbed and tanged point, SF39 and SF40 found with other burnt and unburnt flints in pit F33 (Period III). The barbs are small and almost vestigial with straight sides forming a triangular point below which a wide, round-bottomed tang protrudes. Once again wide morphological parallels may be drawn but little information is added to the site interpretation.

\section{The function of the assemblage}

The lack of evidence for on-site knapping may suggest that the assemblage represents a collection of both retouched and unretouched tools with the waste from alteration processes such as use, sharpening, breakage etc. Of note is the presence of macroscopic edge damage on both flakes and retouched pieces. Like breakage (only two of the retouched pieces are broken), this has many causes, for example both preand post-depositional wear and tear. However, it may also be an indication of use.

\section{Cultural and chronological affiliations of the assemblage}

In general the traditional practice of drawing cultural conclusions from lithic material cannot now be held valid. Cultural parallels must be constructed from careful study of complete assemblages, including 'waste', and even if cultural associations may be demonstrated chronological contemporaneity does not necessarily follow. In Scotland available raw materials have played a large part in determining local technologies and affecting the assemblages produced so that classical culturally determined assemblages rarely exist.

At North Mains, although the assemblage is small, constraints such as raw material and technology have been examined. However, such detail is available for comparison on few other sites and the parallels that the retouched pieces from North Mains do draw are very general and of morphology only. The barbed and tanged point might be considered to be an exception, commonly being held to be indicative of a Late Neolithic/Early Bronze Age date. The type runs through several cultural traditions within this period, however, and it would, in any case, be unsound to base conclusions for a whole site upon a single flint within the flaked stone assemblage.

\section{The distribution of pieces within the site}

The size of the assemblage is too small for patterns of distribution to emerge. However, there is a difference between the type of piece located within the fill of the features and those from the site surface. This difference is most apparent in the distribution of burnt pieces, of which there are a number. The lack of concentrations of burnt pieces upon the 'site surface' probably indicates that they are the result of 
166 | SOCIETY OF ANTIQUARIES OF SCOTLAND, 1983
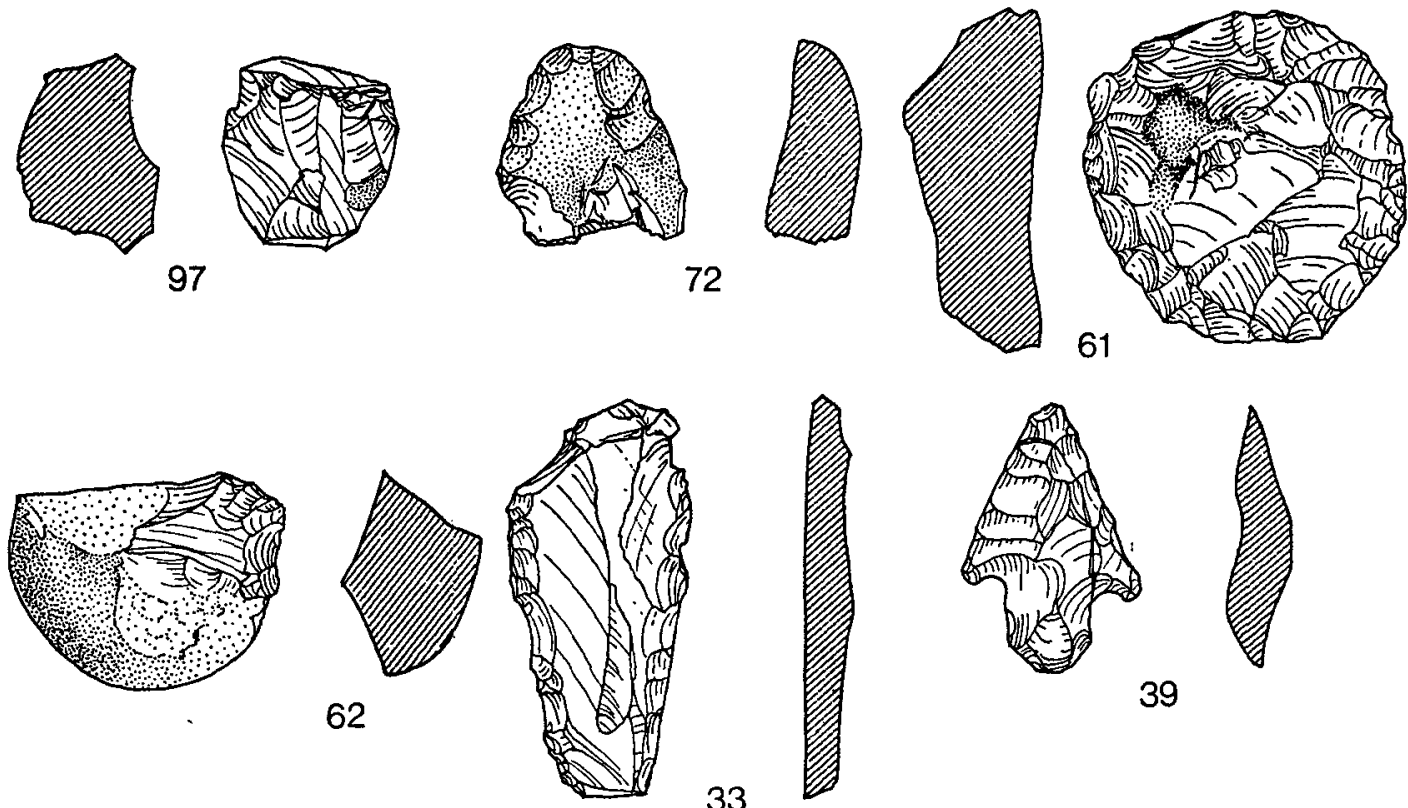

62
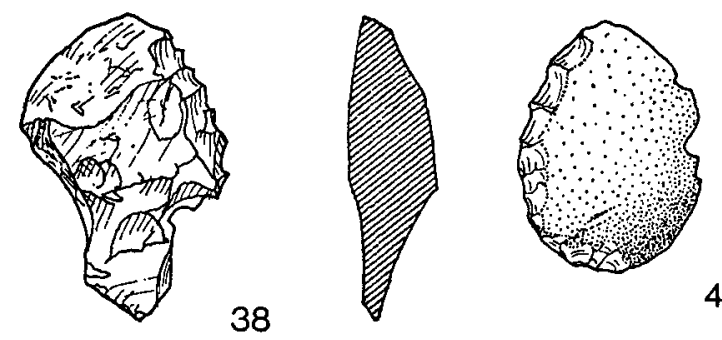

43
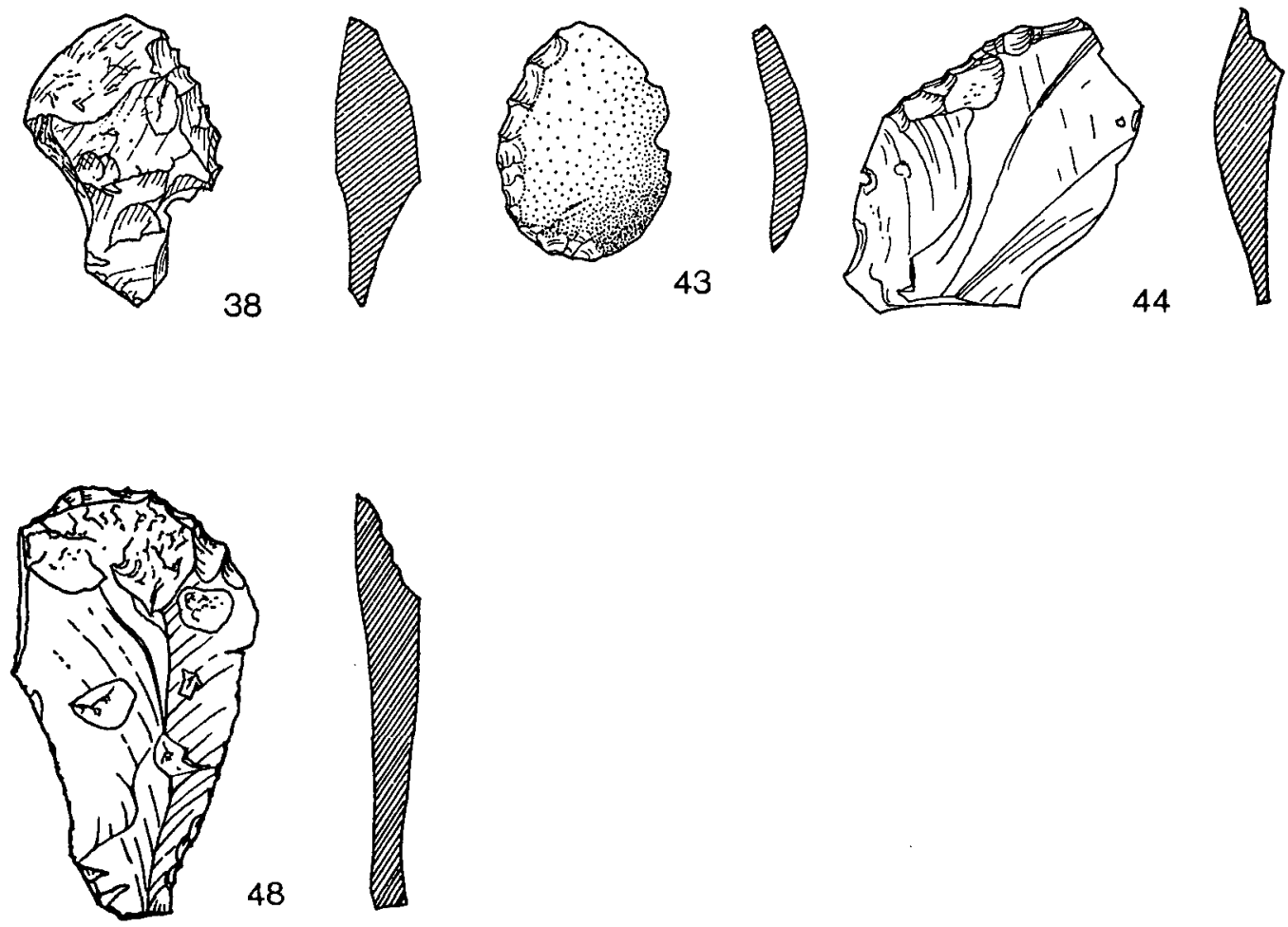

FIo 33 Henge flaked stone (scale 1:1) 
chance burnings and subsequent scatterings. In two cremation burials ( $E$ and $J$ ) and a pit (F33) of Period III, however, there is a much greater concentration of burnt flints though here some are unburnt.

In addition to the burning a higher percentage of retouched pieces is revealed in the Period III assemblage, probably as the result of a more purposeful deposition process in features than upon the 'site surface'. These features have less débitage than the 'site surface' and this would strengthen the argument for a more general scatter upon the 'site surface' as opposed to more ordered deposition within the features.

\section{Summary and conclusions}

A number of different stones are represented amongst the 74 pieces in the assemblage from North Mains. The manufacturers worked predominantly in flint which was probably collected as pebbles from local gravels but there is some evidence of the use of agate and pitchstone and possibly quartz. The pebbles were quite large and of good quality with few flaws, they were made into regularly shaped flakes by percussion with medium and soft hammers upon artificial, sometimes facetted, platforms. Some of the flakes were altered by retouch, occasionally just to create a good edge, sometimes the shape of the whole piece was altered. Many were probably used without secondary working. The retouch is irregular, blanks were chosen primarily upon a basis of size and quality and the individual morphology of the flake affected and shape of the scars. None of these pieces is duplicated within the assemblage and although a few provide parallels with other sites, such parallels are always of general morphology only and can offer no other information.

When the assemblage from North Mains is examined in the context of the site there is little indication of its exact nature. The collection is small and does not contain much evidence that knapping took place on site. The lack of direct flaking evidence, cores and débitage etc, together with the disproportionate amount of inner flakes, might suggest that the pieces were brought ready knapped on to the site as a collection of retouched and unretouched tools some of which were resharpened, altered or broken during use and subsequent deposition. In this way a small amount of débitage was created. No individual functional analysis has been completed in this study so that the actual activities in progress remain unclear. There is no evidence of patterning of any sort in the general distribution of the pieces, which is particularly thin, and a random scatter is most likely. The small number of pieces may suggest that flaked stone was only rarely used or that other factors had resulted in the paucity of such material.

\section{CATALOGUE}

\section{Notes to the catalogue}

i All pieces are flint unless otherwise stated.

ii When examining the pieces they are always held with the dorsal face uppermost and the proximal end towards the observer.

iii Dimensions are given in millimetres in the order; length: width: thickness.

iv Length is measured along a line at $90^{\circ}$ to the platform of the piece, width is in the same plane and at $90^{\circ}$ to the length along a line across the widest part of the flake, thickness is measured from the ventral surface to the highest point of the dorsal surface along a line perpendicular to both length and width. Each measurement gives a maximum reading.

v Chips and chunks have neither a platform nor a ventral surface. The largest dimension of a chunk is over $15 \mathrm{~mm}$, that of a chip is under $15 \mathrm{~mm}$.

vi When classifying the retouched pieces few items are readily paralleled by conventional morphological types and allocation of a name has been left to the end of an entry as this is largely a subjective matter. No functional information is implied by these terms. As conventional type-names infer a degree of function, in the few cases where they might be considered appropriate, they have been placed in parentheses following a morphological type-name.

vii Macroscopic edge damage has been noted where apparent. This generally consists of the removal of small flakes and may be due to use although this cannot be verified without the use of high powered microscope.

viii Cortication refers to the matt discoloration, usually white or cream, which may cover the surface of a flint with time. Patination is the lustrous sheen that may subsequently develop (Shepherd 1972, 114-18).

ix The following abbreviations have been used: 1 , left edge angle; $r$, right edge angle; $p$, proximal edge angle. 
$x$ Cores have been classified in the following manner:

i With a single platform

iia With two opposed platforms

iib With two angled platforms

(My thanks are due to $\mathrm{G}$ Wilson who drew my attention to this method of core classification.)

xi All illustrated pieces are in fig 33.

\section{TABLE 5}

Flaked stone from the henge

\section{PERIOD I}

Under the bank

SF28

SF29-30

Secondary flake

PERIOD II

Ring $A / 8$

SF31

Inner flakes

\section{Bank Material}

SF32

SF33

Inner chunk (quartz)

\section{Secondary flake}

Retouched piece. Inner flake; honey; lightly corticated; broken; proximal surviving; straight sides converging to distal; blunt proximal; irregular retouch on left and right edges; macroscopic edge

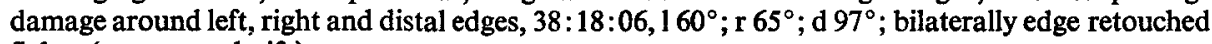
flake; (scraper or knife)

\section{PERIOD III}

F33

SF34-36

SF37

SF38

SF39

SF40

Burial $E$

SF41

Burial $F$

SF42

SF43

SF44

Burial $J$

SF45

SF46-47

SF48

PERIOD IV

F3

SF49

F4

SF50-51
Inner chips (All burnt)

Inner flake

Retouched piece. Inner flake; burnt; pale grey; corticated; irregular shape; remnant retouch on one edge; $30: 22: 08$; edge angle $48^{\circ}$; broken retouched flake

Retouched piece. Inner flake; burnt; pale grey/white; corticated; broken; left half surviving; irregular shape; remnant shallow invasive retouch on both surfaces of straight left side; 22:13:04; $142^{\circ}$; joins with SF40; barbed and tanged point

Retouched piece. Inner flake; burnt; white; corticated; broken; right half surviving; irregular shape; remnant shallow invasive retouch on both surfaces of straight right side; $23: 12: 03 ; \mathrm{r} 55^{\circ}$; joins with SF39; barbed and tanged point

Natural flake (chert)

Inner chip (burnt)

Retouched piece. Primary flake; pale grey; slightly corticated; oval plan; small irregular retouch on left edge; $25: 18: 04 ; 178^{\circ}$; unilaterally retouched flake

Retouched piece. Inner flake; burnt; pale grey/white; corticated; lightly patinated ; artificial platform; soft hammer; irregular shape; remnant retouch on distal end left edge; 30:26:08; $149^{\circ}$; broken retouched flake

Inner chip (burnt)

Inner flakes (both burnt)

Retouched piece. Inner flake; burnt; white; corticated; lightly patinated; prepared platform; straight narrow proximal; straight sides expanding to wide blunt distal; steep remnant retouch on distal end; macroscopic edge damage on left and right edges both ventral and dorsal surfaces;

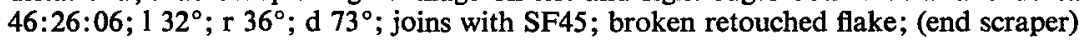

Inner flake (quartz)

Inner flakes (50 burnt) 
TABLE 5

Flaked stone from the henge (continued)

Topsoil and surface collection within henge

(including fill of modern ditch)
SF52 Natural pebble. Agate; red; prominent ring crack on central upper surface marks unsuccessful attempt at splitting; $31: 29: 20$
SF53 Secondary chunk
SF54 Inner chip (burnt)
SF55 Primary flake (quartz)
SF56 Secondary flake
SF57-60 Inner fiakes (59 burnt)
SF61 Retouched piece. Secondary flake; pale grey; partially corticated; circular plan; steep irregular retouch around all edges; macroscopic edge damage around all edges particularly distal; $32: 33: 11$; $160^{\circ}$; r $73^{\circ}$; d $78^{\circ}$; p $69^{\circ}$; discoidal scraper
SF62 Retouched piece. Secondary flake; honey; natural platform; convex proximal, left and right sides; straight distal with projecting beak to right; long shallow retouch on beak; 20:27:12; d $61^{\circ}$; miscellaneous retouched flake

Within excavated area outside the ditch

SF63 Secondary chunk (quartz)

SF64 Inner chunk (burnt)

SF65-66 Inner chips (both burnt)

SF67 Secondary flake

SF68-71 Inner flakes (68 pitchstone; 70 banded agate)

SF72 Retouched piece. Primary flake; pale grey; corticated; broken; distal surviving; straight left and right sides converge to blunt point at distal; shallow irregular retouch on left edge and around distal; $18: 18: 08 ; 170^{\circ} ; \mathrm{d} 82^{\circ}$; broken retouched flake

Ditch, secondary fills and topsoil

SF73-75 Natural flakes (all chert)

SF76 Primary chunk (quartz)

SF77 Secondary chunk

SF78 Secondary chip (burnt)

SF79-85 Inner chips (79-80 burnt; 81-85 pitchstone)

SF86-87 Primary flakes (86 pitchstone)

SF88-89 Secondary flakes (89 burnt)

SF90-96 Inner flakes (93 pitchstone; $92,95,96$ burnt)

Surface collection outwith site

SF97 Core (type iib)

SF98 Secondary chunk (pitchstone)

SF99 Secondary flake (pitchstone)

SF100-101 Inner flakes

\section{OTHER ARTEFACTS}

\section{G J Barclay}

SF102 (Ring A/3; fig 34) A D-shaped portion of a larger slab of diorite measuring between 54 and 61 $\mathrm{mm}$ thick, a maximum of $208 \mathrm{~mm}$ long and $95 \mathrm{~mm}$ broad. Both faces show signs of some smoothing. The stone may have been used as a grinding or rubbing surface.

SF103 (LG1; fig 34) A portion broken from a larger slab or red sandstone. The undamaged part measures a maximum of $235 \mathrm{~mm}$ broad, $360 \mathrm{~mm}$ long and $62 \mathrm{~mm}$ deep. The upper surface is dished and smoothed with one well defined (? sharpening) groove visible on it. The slab had been used for rubbing or grinding. After being broken it was deliberately deposited at the W end of LG1, where it probably acted as a rest for the head of the inhumation.

SF104 (F42) A part of a larger slab of red sandstone measuring a maximum of $250 \mathrm{~mm}$ long, $155 \mathrm{~mm}$ broad and $100 \mathrm{~mm}$ deep. The upper surface has been smoothed. The artefact was probably used for grinding prior to its insertion in F42, where it may have acted as a post support.

SF105 (Top ditch fill) An irregular piece of micaceous green sandstone. Approximately half of a smoothed surface survives on the only flat face; the remainder of the stone has not been worked. 

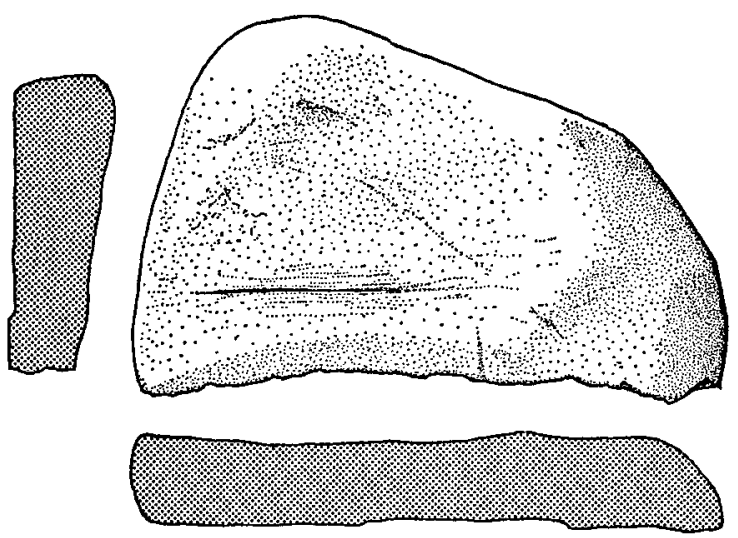
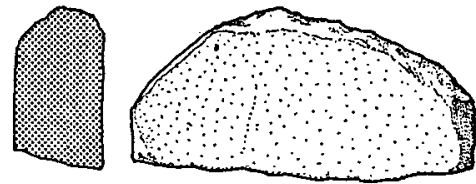

102
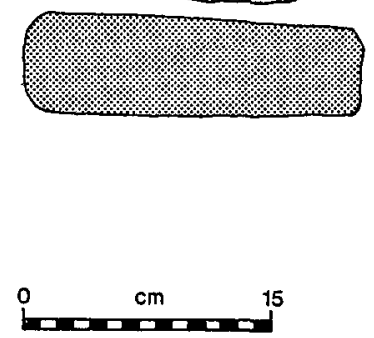

FIG 34 Henge stone

The flat surface measures a maximum of $108 \mathrm{~mm}$ long, $72 \mathrm{~mm}$ wide and $95 \mathrm{~mm}$ deep. Despite its irregular shape the stone fits conveniently into the hand to allow the flat surface to be used.

SF106 (Topsoil) A piece of siltstone of rectilinear section $16 \mathrm{~mm}$ long and between 41 and $51 \mathrm{~mm}$ across. All four sides are very smooth; both ends are slightly smoothed suggesting use as a whetstone or hone.

SF107 (Topsoil) A piece of micaceous red sandstone measuring $270 \mathrm{~mm}$ in length with a rhombic section. One edge is very rounded; the others are sharply defined. One end is relatively pointed and shows slight signs of wear. The other end is flat.

\section{CREMATED BONES FROM THE HENGE MONUMENT AT NORTH MAINS}

\section{R Powers, Department of Palaeontology, British Museum (Natural History)}

All the bones submitted were fully cremated. Much of the material had been so warped and fractured by the heat as to be virtually unrecognizable. Therefore only the identifiable parts have been plotted on the standard skeletal outlines illustrating this report (figs 35-37), though exact determination as to left and right is not always certain. The sex identification as 'male' may be reasonably certain because of the dense muscle-attachments which survive cremation well, but 'female' is mainly identifiable by default. A few were too fragmentary, or too well-burnt, even to guess at the sex.

Many of the smaller cremation deposits did not look human; though strictly speaking indeterminate, the bone texture and general appearance was wrong. Fortunately several of these deposits provided indubitable quadruped bones - usually from the extremities. (Extremity bones survive well in the human material too, probably because they fall off at an early stage and are protected in the ash.)

The animal bones found in the smaller cremation deposits were submitted to Barnetson for further identification. References to Barnetson's report are marked (LB), in the full entries, or are listed at the end of the report.

\section{CATALOGUE}

\section{Burial $A$ (fig 35) $741 \mathrm{~g}$}

Young adult. Probably male aged 20 years. Skull: About 20 vault fragments $\mathrm{c} 1 \mathrm{~cm}$ across, some showing open sutures, and one larger piece which contains the nuchal muscle ridge (inion) moderately well developed. The face is represented by a scrap of the left maxilla and some 10 tooth fragments, some of which are molar roots. Post-cranial skeleton: These are mainly long-bone fragments, including tibia anterior ridge and linea aspera of femur, (neither very robust), and a scrap of fibula shaft. The arms were also represented. A proximal epiphysis of the humerus shows that it was not united in the middle to the diaphysis. This suggests the age. Fragments of the distal articulation (representing both elbows) were 

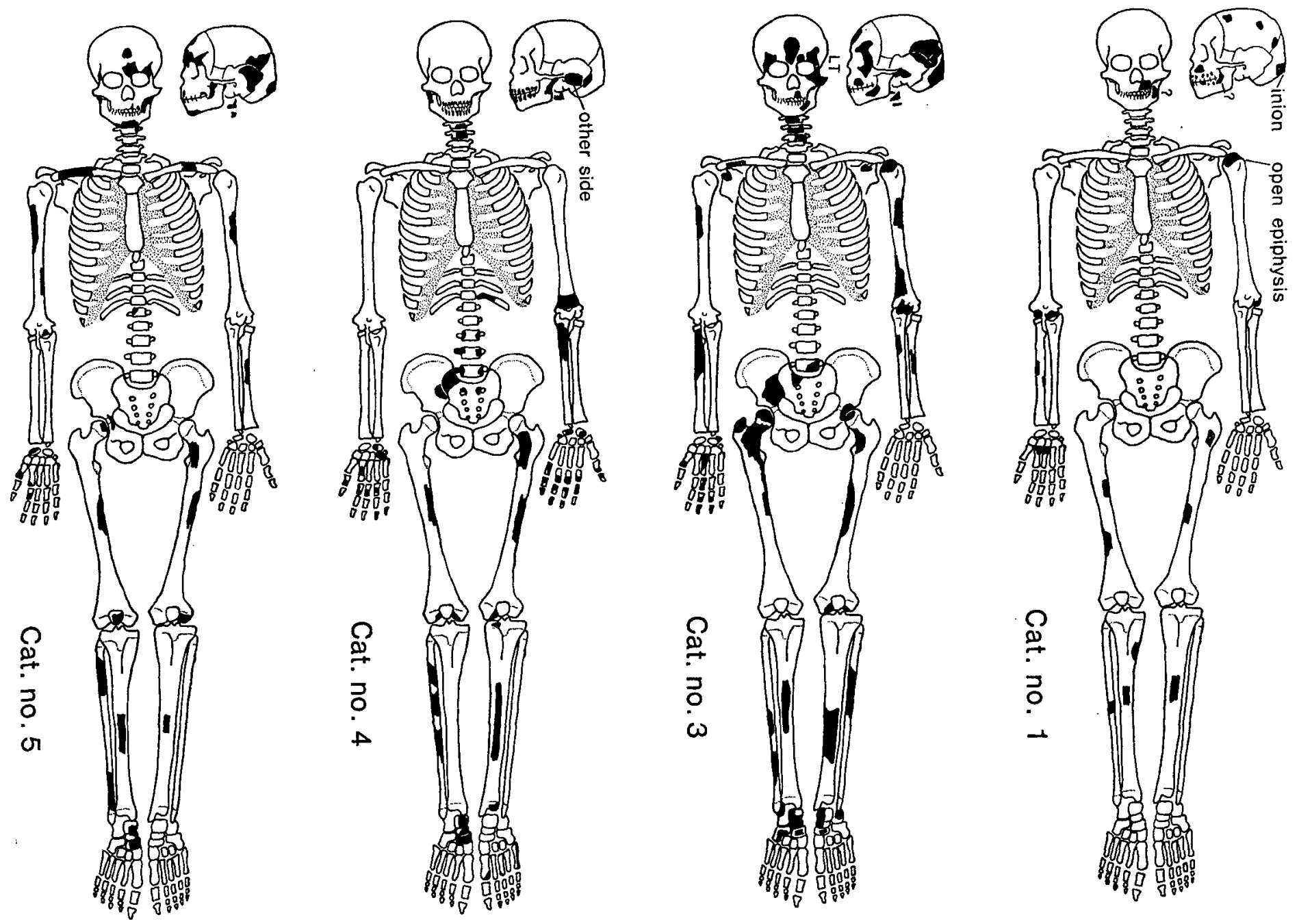

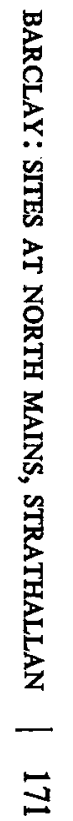


present. The bases of two metacarpals, probably one and two, represent the hands. Nothing recognizable remained of the trunk.

\section{Cremated portion of Burial D (LB) $55 \mathrm{~g}$}

Age juvenile, but species not necessarily human. Skull: Fragments of thin skull with remains of ossa petra, and one unerupted calcified 'tooth germ' which may be a canine. Post-cranial skeleton: Fragments of thin-walled long-bones and part of a cervical vertebra arch.

\section{Burial F (fig 35) $1415 \mathrm{~g}$}

Young Adult male. Skull: A few teeth and part of the left malar bone represent the face. A small piece of alveolar border with sockets for a canine and two premolars is probably left maxilla. The surviving part of the mandible shows a socket for the rear molar on the left (either M2 or M3). Both outer corners of the frontal bone and part of the inner table showing the midline ridge prove it was not metopic. Most of the left orbital border of the frontal exists, and supraorbital foramena (not notches) were present on both sides. The remaining portion of the frontal sinus suggests it was fairly extensive (male). The largest surviving area of the skull is the left occipito-parietal around asterion. Both jaw joints, sockets and bits to temporal bone show robust muscle attachments. The inion is a single triangular lump not extending in a bar across the occipital like catalogue nos 3 and 5 . The surviving sutures, mainly round asterion, were open. Teeth: a few teeth remain. Post-cranial skeleton: Many long-bone fragments including recognizable parts of all main long-bones, also scraps of the shoulder and pelvic girdles from protected areas. The right sciatic notch area is remarkably complete for a cremation and has no trace of a sulcus, which reaffirms the sex as male. Parts of atlas, axis and three cervicals, plus a few other vertebral fragments represent the spinal column. Some bones of the tarsus of both feet are present, certainly both astragali, but not part of the fore-foot. Parts of both hands are represented. The base of metacarpal three is duplicated.

\section{Burial G (fig 35) (LB) $2784 \mathrm{~g}$}

Adult male; probably under 35. Skull: Part of the zygomatic arch and a (right ?) mandibular condyle represent the face. Vault fragments $2-3 \mathrm{~cm}$ across were present, representing parietal, occipital and temporal bones at least; the temporo occipital, lambdoidal and sagittal sutures were open. The muscle ridges of the temporal and occipital were well marked. Teeth: So many tooth roots were found as to arouse suspicion that more than one individual was represented but this could not be proved by duplication of parts. Post-cranial bones: All major long-bones are represented with the possible exception of the radius. The axis body with odontoid and parts of two other cervicals are present. The right wing of the first sacral arch and the adjoining area of acetabulum (unfortunately not including the sciatic notch) were recognized. Hand and foot bones were more easily identifiable, they include the inner border of the right talus and navicular and the outer border of the left fifth metacarpal. Carpals including hamate and pisiform of both hands and many finger phalanges survive.

\section{Burial $H$ (fig 35) $1402 \mathrm{~g}$}

Adult male. Skull: Left coronoid process of mandible, both condyles, chin, two tooth roots all preserved as separate fragments. The cranium is represented best by the left orbital margin of the frontal bone (which is not metopic) and by the parts around the left mastoid area. Inion is also present: it and the mastoid area show male robusticity. Post-cranial bones: The outer parts of the clavicles are recognizable though calcined, the rest of the trunk only minute vertebral fragments. All major long-bones are represented by shaft splinters. The hand is represented by a scaphoid and distal articulations of two metacarpals and phalange. The tarsus is represented by parts of the talus and navicular on the inner side of the foot.

\section{Burial J (fig 36) $1656 \mathrm{~g}$}

Adult male, probably under 35, and child 4-6 years, well mixed. Adult Male: Skull: Two (?) fragments of maxilla and four of mandible, showing sockets for teeth (incisors, premolars and molars), also loose teeth, including at least two molars. The mental tubercles are present, duplicating this area in the child. Vault fragments represent all major skull areas, especially the occipital which is preserved for its full width and has a well marked nuchal muscle insertion. Robust muscle markings on temporal and frontal confirm the sex as male and part of a large frontal sinus remains. The vault sutures were open and all teeth probably present indicating youth. He was not metopic. Post-cranial bones: Shaft fragments repre- 

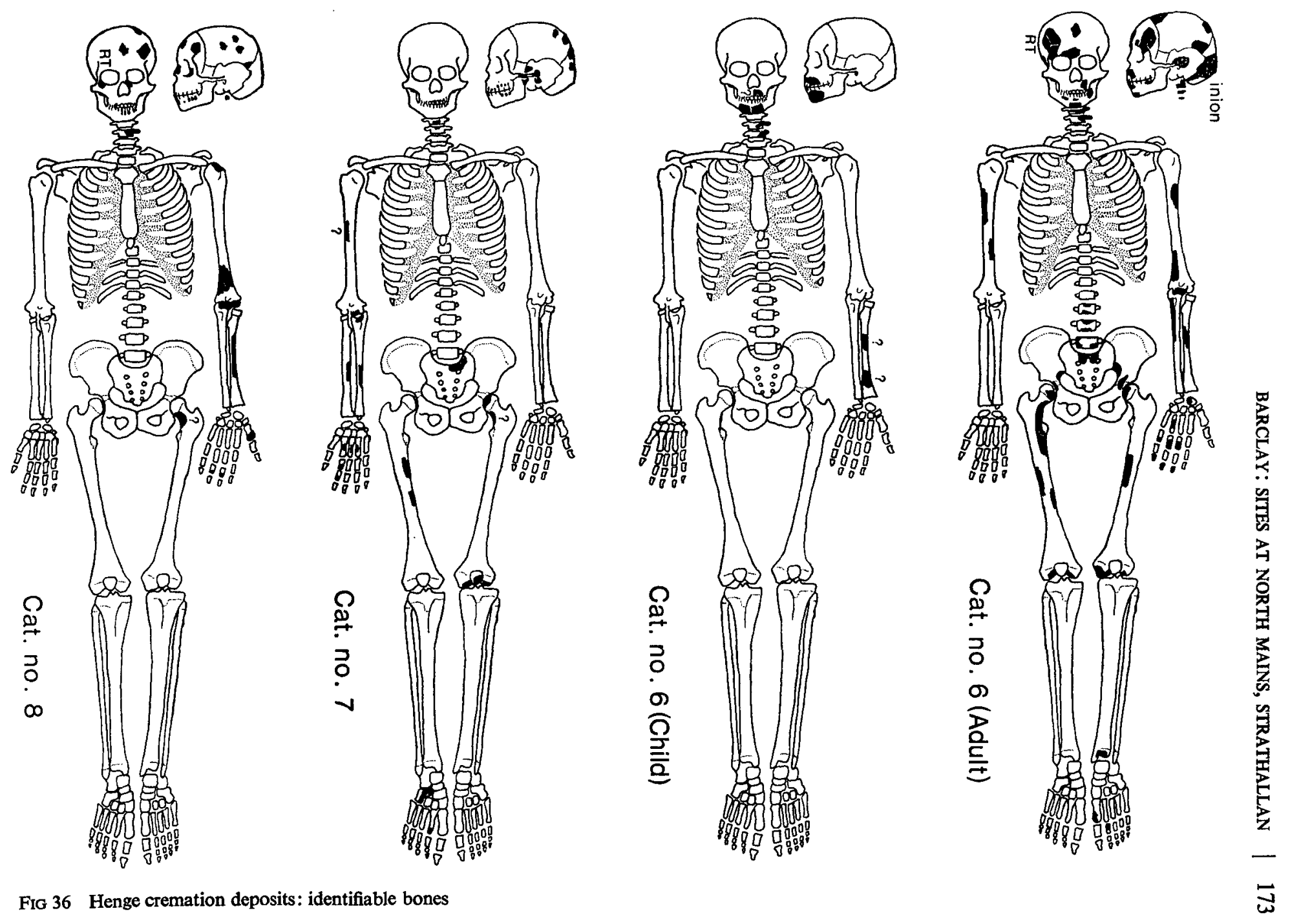

FIG 36 Henge cremation deposits: identifiable bones 
senting femur, humerus and ulna were robust and masculine; all major long-bones were represented. In addition portions of hip and knee articulations survived and similarly parts of vertebral body articulations. One vertebral fragment, or perhaps first sacral segment, shows roughening suggestive of early osteoarthritis. There are also recognizable fragments of the sciatic notch area. The foot is represented by two fragments; proximal end of basal phalange and distal end of great toe metatarsal. The hand is better represented, a left hamate-hook, part of a metacarpal, three terminal phalanges and three distal ends of phalanges, all of fingers.

Child: Identifiable by mandible. Sockets for deciduous incisors, canines and first (milk) molars are present, also the unerupted crowns of a canine (half-formed) and lateral incisor. The first permanent molar crowns were probably formed but unerupted (to judge by the remains of their sockets which were broken through). Part of a left maxilla may more probably belong to the adult. A number of thin skull vault fragments with open sutures survive. The post cranial-skeleton is represented only by three fragments of cervical vertebra arch and three more of very slender long-bone; but at that age it is frail and was probably completely burnt.

\section{Burial K (fig 36) $221 \mathrm{~g}$}

Fairly young adult, perhaps female. Preservation: The bone was pale cream in colour and fully cremated. One small group of fragments had a grey-blue tinge, especially inside the marrow-cavity and may represent one bone subsequently fragmented. The teeth also show a greyish tinge. Most of the bone was too fragmentary to identify but some areas, notably of the feet, are recognizable. Skull: A piece of zygomatic arch, some teeth and a probable mandibular condyle represent the face. The teeth are crown fragments of probably canine and premolar, and root fragments probably from molars. The dental canals are closed; growth was therefore completed. The skull vault fragments show only open sutures (lambdoid and sagittal) and a piece of the porion area of the temporal is recognizable. Post-cranial skeleton: The trunk is represented by the dorsal spine of a cervical vertebra and part of the anterior aspect of the first sacral segment. Part of the radius and adjoining articular areas of ulna and humerus are present. Of the hands part of the pisiform and distal ends of two phalanges and some metacarpal remnants survive. The usual fragments of the densest parts of the femur survive but are not particularly robust or muscular. More than half of one patella is present, also part of the distal articular surface of the femur and a scrap of its head. Medial half of left outer cuneiform and distal ends of three metatarsals and a terminal toe phalange represent the feet.

\section{Burial L (fig 36) $482 \mathrm{~g}$}

Adult, perhaps female. Skull: Vault fragments up to $2 \mathrm{~cm}$ across and rather thin with open sutures (arousing a suspicion that it was immature except that no open epiphyses were found). Frontal and parietal fragments were identified. Fragments of orbital rim of right malar bone and left nasal process of maxilla and some teeth represent the face. Both anterior and posterior teeth are present. Post-cranial skeleton: Odontoid process of axis and part of another cervical vertebra represent the neck. The humerus is represented by proximal and distal articulations and shaft-splinters; the radius by shaft-splinters only; the hand by distal articulations of phalanges and of a thumb metacarpal. Nothing certainly identifiable as from the lower half of the body is present. A fragment identified on the diagram as femur neck could be from the corresponding part of the humerus instead.

\section{Burial $M$ (fig 37) (LB) $811 \mathrm{~g}$}

Adult aged c 30 and perhaps female. Skull: All main vault bones are represented. Some of the surviving skull fragments show open sutures; the rear part of the sagittal is fusing but the rest appear to be open. This probably includes the metopic suture. The upper part of the left malar and adjoining corner of the frontal bone survive, but this malar fragment is duplicated, indicating two individuals. This is the only duplicated part in the find and may be an accidental inclusion. The anterior part of the left maxilla with sockets for lateral incisor and canine (and three fragments of tooth root probably representing them) are present. The dentine of half a crown shows that attrition had not worn through the enamel: Postcranial bones: Shaft fragments of all main long-bones except perhaps ulna and fibula are present, and protected areas inside the joints at elbow (ulna/humerus articulation) and hip (acetabulum and femur head). Part of a patella represents the knee. A scrap of scapula and, unusually, a vertebral body, probably thoracic, and bits of other vertebrae, including a cervical, represent the trunk. The pattern of survival suggests that the left side of the head lay on the ground and the thorax was perhaps protected by something 

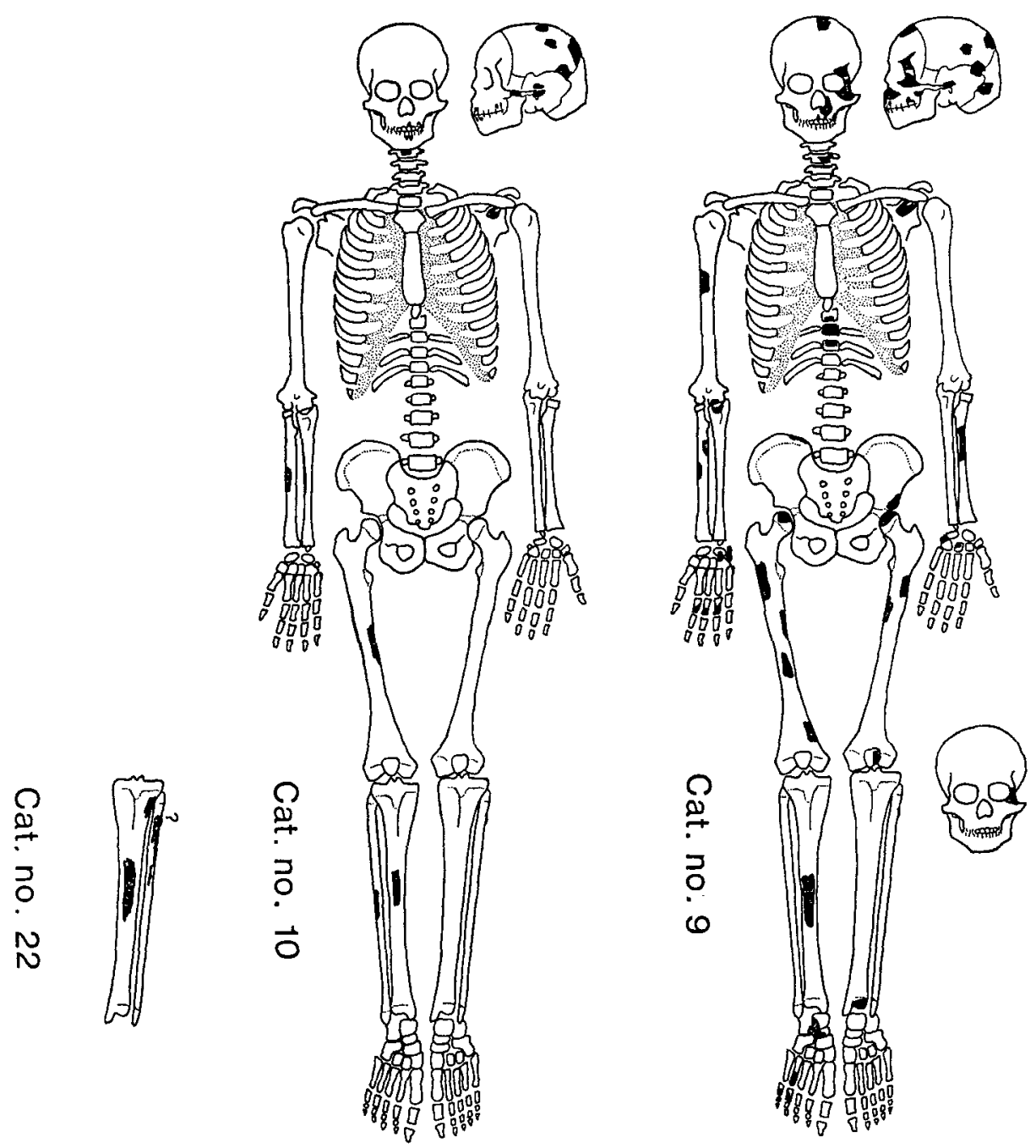

FIg 37 Henge cremation deposits: identifiable bones

during the burning. Two hamate-hooks, two pisiforms, and three distal articulations of phalanges represent the hands. Scraps of the contacting articular surfaces of calcaneum and astragalus, the distal end of a metatarsal, the upper aspect of one medial to phalange and the distal articulation of another, and the two fused phalanges of a 'little toe' represent the feet. The lack of robustness suggests the sex is female.

\section{Burial $O$ (fig 37) $308 \mathrm{~g}$}

Adult, over 30 years old. Skull: A number of cranial fragments including parts of right porion area, zygoma, and petrous of temporal, and vault including rear part of sagittal between parietal foramena and lambda. The lambdoid suture was therefore open but the sagittal was obliterated internally while still visible externally. Other vault fragments of parietal and occipital show only open sutures. Three fragments of tooth roots are also present. Post-cranial skeleton: These were mainly of long-bone shaft including radius, fibula and femur fragments. Part of the arch of a cervical vertebra and a piece probably from a scapula were also recognized. Miscellaneous Material: Although this was assumed to be human, the lack of thick cranial fragments, femora and other characteristic pieces of human cremations makes one suspect a small quadruped (deer, sheep or pig) as alternative source. 


\section{Ring $B / 5 \quad 10 \mathrm{~g}$}

Fragments of medium-sized long-bone shaft.

\section{Ring $A / 7$ (LB) $73 \mathrm{~g}$}

Fully cremated, white and somewhat rolled fragments. Long-bones of various diameters are represented. One tooth without crown is present, a single-rooted, stout, tooth which shows no trace of the dental canal at root tip. It could well be an artiodactyl tooth. A fragment of small triangular sectioned long-bone shaft shows a ridge which might be callus from a fracture-repair.

\section{Ring $A / 18 \quad 5 \mathrm{~g}$}

Dense, white, probably long-bone cortex.

\section{F33 $12 \mathrm{~g}$}

These are very well-burnt, and except that some five appear to be skull-vault fragments they are indeterminate. The colour is white with black superficial stain. A thin line of diploe is visible on the best preserved vault fragment.

\section{1. $549 \quad 7 \mathrm{~g}$}

Two pieces might be from the lower border of the chin of a mandible. It is possible that the smaller pieces are part of the same bone. But this interpretation is very dubious.

\section{Burial $N$ (fig 37) $29 \mathrm{~g}$}

The material is probably human, and probably adult. A fragment of burnt shell adhering to the bone was peeled off (see p 149). Two pieces contact and were united to make a sliver from the anterior ridge of a tibia, straight as in human. Several of the larger pieces could be from the upper, lateral parts of the same bone, where it thins and curves out to form the knee. Three very small skull fragments may be human. The alternative of a small bovid cannot be quite ruled out.

32,33 37-45. These small fragments of human bone were found on the surface in and around the henge and in the modern fills of the ditch. The total weight was $67.5 \mathrm{~g}$.

The following are non-human or indeterminate:

Catalogue numbers 13 (Ring A/8); 14 (Ring A/10); 16 (Ring A/15); 17 (Ring A/16); 18 (Ring A/17); 23 (LG2); 24 (LG7); 25 (LG10); 26 (Burial A; backfill); 27 (Burial B; backfill); 28 (F3; Burial P); 29 (F4); 30 (F5; Burial Q); 31 (F29); 34; 35; 36; $46 ; 47$.

They were submitted to Barnetson for further identification, successful only in the case of nos 13, 16, 17,18 and 29, in addition to the animal bone incorporated in burials $D, G$ and $L$, and in Cat No 12 .

\section{UNBURNT HUMAN REMAINS FROM THE HENGE MONUMENT AT NORTH MAINS}

\section{A Young and D A Lunt}

These two originally separate reports have been combined. Dr Lunt has reported on the jaws and dentition, Dr Young on the remainder of the skeletons.

\section{CATALOGUE}

Burial $B$ (fig 15, p 136)

The following skeletal elements were represented in varying degrees of completeness:

Skull: There were several loose pieces of calvarium. Calvarial sutures were not fused internally or externally. The frontal sinuses were well formed, but the supraorbital ridges were not prominent and the forehead region sloped back quite markedly. The surviving mastoid process was not large. Both the frontal and left parietal bones showed areas of erosion of the outer table. 
Jaw and dentition: The palate and part of the alveolar process of the maxilla have survived, and the body and left ascending ramus of the mandible are present. All 16 permanent teeth are in situ in the mandible. In the maxilla, eight teeth are still in place in the alveolus and the remaining eight teeth have been recovered but cannot be returned to their sockets. Thus a complete permanent dentition is present. All the teeth have erupted into functional positions, and are perfectly arranged without crowding in broad, well-formed dental arches.

$\mathrm{X}$-rays show that the root apices of the third molars have been completely formed, and these suggest that the individual was probably over 20 . The degree of molar attrition is relatively slight, indicating a young adult, perhaps between 20 and 25 years of age. The teeth are well-formed and there is no evidence of hypoplasia. Neither dental caries nor periodontal disease has affected the dentition.

Post-cranial skeleton: Vertebrae: Cervical - first, second and another. Thoracic - 10 in all. Age probably between 22 and 25 years: fusion of upper and lower epiphyseal rings to vertebral bodies was incomplete. Sacrum: Part of first segment. Ribs: Various pieces, including a left first rib. They are not of heavy build. Scapulae: Right and left. Clavicles: The outer halves of both right and left. The right one had probably been fractured at some time, and the left one showed a 'canal' for supra-clavicular nerve. Humerus: Most of a right with the epiphyses fused and therefore an age of 22 years or more. Radius: Proximal end of a right. Ulna: Upper part of a right. Metacarpal: Part of one. Phalanges: Three carpal phalanges. Innominate bones: Left ilium and two other pieces. Femora: Lower parts of right and left epiphyses are fully fused therefore age over 22 years. Tibiae: Upper parts of right and left. Patella: Fragment of left (?). There were also many unidentifiable bone fragments.

Sex, age and height: There were no suitable intact bones from which to estimate the height. On the whole this was probably a female of about 22-25 years.

\section{Long Grave 3}

Skull: Of this there remained a large piece of the left side, comprising the left parietal and left temporal bones and the left side of the frontal bone; part of a right temporal bone and the torcular portion of the occipital bone were separate. The left mastoid process is small, the calvarial elements are not thick and the sutures are not closed.

Jaw and dentition: The only recognizable fragments of the skull are the left condyle of the mandible and a portion of the left ascending ramus and body of the mandible with three permanent molars in situ. Another 13 permanent teeth are present, all but one in a fragmentary condition, having suffered severely from post mortem destruction of the dentine. Some further teeth are represented by unidentifiable fragments of enamel.

In the left molar region of the mandible is observed the unerupted third molar still lying deep in the bone behind the second molar. An X-ray shows that the third molar is only partially formed: this stage of development would normally be reached between 13 and 17 years. The root apices of the second molar are completely formed, and this generally occurs between 14 and 16 years. The most probably age at death would therefore be approximately 14-17 years. The slight degree of attrition of the first and second permanent molars supports this estimate. The teeth are well formed and do not show hypoplasia. There is no evidence of dental caries.

Post-cranial skeleton: Sacrum: Parts of the first two sacral segments showed early fusion of the lateral masses on the left side but the centra are still clearly separate. This would indicate an age around puberty, say 14-16 years. Humerus: Possibly the upper end of a humerus. Metacarpal: Part of a metacarpal shaft. Innominate bones: Portions of both right and left iliac bones were present. Femora: Represented by part of the shaft and neck of a right femur, the upper end and part of the shaft of a left femur and the lower ends of a right and a left femur. As all femoral epiphyses were fused the age was likely to be at least 20 years, though for female one might lower this estimate slightly. Tibia: Part of the upper end of a tibia (? right). Fibula: Part of the shaft of a fibula (?). Patellae: One almost complete, and part of another.

Sex and Age: The individual was probably a young female. If the femora do belong with the skull, teeth and sacrum, then one must take the upper end of Dr Lunt's estimate and suggest an age of 17 years at least. If one discounts the femoral eipihyseal evidence then the age would lie in the range of 14-16 years. 
The femora indicate an individual of small build but as no suitably complete long bones were present the height could not be computed.

\section{Long Grave 7}

The single piece of bone which remains may be from the basal part of an occipital bone.

\section{IDENTIFIABLE BURNT ANIMAL BONE FROM THE HENGE MONUMENT AT NORTH MAINS}

\section{P D Barnetson, Department of Archaeology, University of Edinburgh}

Positive identification to bone and species was possible in only a few cases and the majority of fragments were not identifiable. All that can be stated is that those bone fragments which were identified belong to artiodactyla, of varying size and age, and apart from one or two fragments, identification to Family (Cervidae, Bovidae or Suidae) could not be made. 'Small artiodactyl' could be sheep, goat, pig, roe deer and 'large artiodactyl' could be cattle or red deer. Fragments submitted which could not be identified have been omitted. 'Cremation catalogue number' refers to Powers's catalogue.

IDENTIFICATIONS

Cremation

Cat No

2 (Burial D)

4 (Burial G)

9 (Burial $M$ )

12 (Ring A/7)

13 (Ring $A / 8$ )

16 (Ring $A / 15)$

17 (Ring $A / 16$ )

18 (Ring $A / 17$ )

$29(F 4)$

\section{Description}

Four tiny fragments of pig teeth.

Two fragments: one almost complete ulnar carpal, ovicaprid. One fragment of trochlear notch of ulna, possibly pig.

One virtually intact, though slightly distorted, cuboid bone (centrotarsal) - ovicaprid.

One fragment of ischium, acetabulum fused (?) of small artiodactyl. One fragment of cranium, possibly small artiodactyl.

Many very tiny fragments of teeth including six larger fragments identifiable as pig molar. Also 27 fragments of cremated bone as follows: 13 long-bone, of which five pieces identified as small artiodactyl humerus, radius and metatarsal diaphyses. Four flat bones, of which one is cranium. One fragment of trochlear notch of ulna. Four rib fragments, small artiodactyl. One fragment of proximal first phalanx of large artiodactyl (probably cattle).

Mostly fragments of long bones, including a possible distal fragment of humerus, large artiodactyl. Four rib fragments, small artiodactyl. One proximal epiphysis of first phalanx of pig (immature). One fragment of distal end of a first phalanx, small artiodactyl. Two mandible fragments, small artiodactyl. Proximal epiphysis of a calcenium - pig (immature). One fragment of proximal epiphysis of a first phalanx - small artiodactyl (immature). Pieces of long-bone of small artiodactyl present.

Pieces of long-bone recognizable, leg bones mostly, and also some fragments of cranium. Both large and small artiodactyla.

\section{THE POLLEN ANALYSIS OF DEPOSITS IN A FOOD VESSEL FROM THE HENGE MONUMENT AT NORTH MAINS}

\section{S Bohncke}

\section{INTRODUCTION}

Burial B was accompanied by a food vessel (SF17), discovered lying on its side by the skull. Within the pot a small deposit of black greasy material was found. As the analysis of material within vessels has proved valuable (Dickson 1978) samples of this deposit and scrapings from the pot wall were subjected to pollen analysis.

\section{MATERIAL AND METHODS}

Part of the black greasy material was used for the preparation of two pollen samples and another sample was scraped from the inner fabric of the pot. All samples were treated with hydrogen fluoride to 
remove the silaceous component and were subsequently subjected to Erdtman's acetolysis. Microscope slides were then prepared using glycerol jel as an embedding medium.

\section{RESULT AND DISCUSSION (table 5a)}

High percentages of Filipendula (meadowsweet) pollen (74.1\%,77.8\%,84\% (scraping)) and relatively high percentages of cerealia pollen were noted. There was also a large proportion of charcoal fragments. Within the Filipendula grains there was a wide range of variation in the states of preservation. The well preserved type is the tricolpate micro echinate pollen in which the pores are clearly pouting. The very corroded type lacks the characteristic pores but the wall structure is not affected. Immature pollen was also present. Relatively high proportions of Filipendula can be attributed in part to selective corrosion as Filipendula has a relatively thick wall but this could not explain the $74 \cdot 1-84 \%$ found in these samples.

TABLE 5a

Pollen analysis of deposit and scraping from food vessel SF17

\begin{tabular}{|c|c|c|c|}
\hline & $\begin{array}{c}\text { Sample } 1 \\
\text { Scraping } \\
\%\end{array}$ & $\begin{array}{c}\text { Sample } 2 \\
\text { Black greasy deposit } \\
\%\end{array}$ & \\
\hline TREES & $\cdot$ & & \\
\hline Pinus & $0 \cdot 2$ & - & Pine \\
\hline Betula & $0 \cdot 2$ & $0 \cdot 2$ & Birch \\
\hline Quercus & $0 \cdot \overline{4}$ & $0 \cdot \overline{2}$ & Oak \\
\hline Corylus & $3 \cdot 2$ & $1 \cdot 0$ & Hazel \\
\hline Alnus & $1 \cdot 1$ & $0 \cdot 2$ & Alder \\
\hline \multicolumn{4}{|l|}{ HERBS } \\
\hline Ericaceae & $0 \cdot 2$ & - & Heather \\
\hline Plantago lanceolata & $0 \cdot 7$ & $0 \cdot 3$ & Ribwort \\
\hline Cyperaceae & - & $1 \cdot 2$ & Sedges \\
\hline Gramineae & $8 \cdot 1$ & $4 \cdot 0$ & Grasses \\
\hline Rosaceae (Crataegus-type) & 0.2 & . & Hawthorn-type \\
\hline Linum usitatissimum & - & $0 \cdot 2$ & Flax \\
\hline Cruciferae & $0 \cdot 2$ & - & \\
\hline Ranunculaceae & $0 . \overline{4}$ & 0.9 & Buttercup \\
\hline Compositae (Tubuliflorae) & $2 \cdot 7$ & $3 \cdot 7$ & \\
\hline Filipendula & $66 \cdot 6$ & $74 \cdot 1$ & Meadowsweet \\
\hline cf Filipendula & $8 \cdot 1$ & $8 \cdot 0$ & \\
\hline Cerealea & $1 \cdot 4$ & $0 \cdot 3$ & Cereals \\
\hline \multicolumn{4}{|l|}{ FERNS } \\
\hline Polypodum & 0.4 & 0.5 & \\
\hline Monoleet psilate-type & $2 \cdot 9$ & $0 \cdot 7$ & \\
\hline \multicolumn{4}{|l|}{ MOSSES } \\
\hline Sphagnum & 0.4 & - & Bog moss \\
\hline INDETERMINATE & $2 \cdot 7$ & $4: 9$ & \\
\hline
\end{tabular}

Cereals are self pollinating, the result being that little pollen is dispersed into the air. The anthers remain within the spikelets so that on the fully grown cereal pollen grains could still be present, though most would be lost during the winnowing process. Compared with the other species the cereals are over represented in both samples. This is probably the result of human interference. The charcoal in the samples might have originated in the processing of the grain (eg roasting to remove chaff), however the cereal pollen were not burnt and this therefore seems unlikely. The charcoal may also have found its way into the pot during the burial ritual or have been in the pot already, perhaps as the result of firing a new vessel for the burial. The remaining pollen almost certainly came accidentally into the food vessel and represented a part of the contemporary pollen rain. The presence of pollen of plants flowering at different times of the year (eg Corylus March-April, Polypodium August-September) might be explained by the addition of pollen-bearing water to whatever was contained in the vessel or its incorporation in the manufacture of the contents.

Dickson (1978) in his analysis of the Ashgrove beaker scrapings discovered very high proportions of Tilia and Filipendula and concluded that the vessel had contained lime honey or mead flavoured with meadowsweet. The North Mains food vessel contained neither lime pollen nor sufficient pollen from other 
honey-producing plants. The $2.43 .7 \%$ of Compositae pollen were not sufficient for a honey based on Compositae alone. The high proportion of cereal pollen grains might suggest either a porridge of cereals (eg frumenty) or a fermented ale, flavoured with meadowsweet flowers or extract. The name 'meadowsweet' orginates from mead-sweet as it was used to flavour mead and other drinks. It is distinctly possible therefore that the North Mains food vessel contained a fermented drink.

\section{DISCUSSION}

\section{G J Barclay}

The reader is referred to the following works which mark major milestones in henge studies: Piggott 1948, Atkinson et al 1951, Burl 1969, Wainwright 1969 and Catherall 1974, 1976.

\section{PERIOD I}

Little can be said about the cultivation of the land prior to the erection of the Period II bank. The disturbance of the fossil soil to a depth of $0.12 \mathrm{~m}$ as demonstrated by clay translocation may be the result of ard cultivation (Romans \& Robertson infra p 267). It has been suggested that the gleyed fossil topsoil may have been formed by the activity of stock, as under the barrow, though probably less intensive. Pollen analysis of the buried topsoil has provided little information on the environment prior to bank construction. Hulme and Shirriffs ( $p 270$ ) have suggested that the Neolithic and Early Bronze Age landscape was not dissimilar to the present day's: an open landscape but with substantial wooded areas.

The features sealed under the Period II bank cannot be dated other than by that relationship. F1 and F7 preceded the formation of the mired old land surface; burial A may have. However, all these features could have predated the construction of the bank by either a long or relatively short time. It could not be determined for what purpose F1 or F7 was dug. It is possible that they were also burial pits, and that the three features represent a tradition of burial perhaps associated with the first ritual use of the area. Burial A may be a lone interment but perhaps burials $\mathrm{L}$ and $\mathrm{M}$ were broadly contemporary; however, the occurrence of the unaccompanied cremation rite throughout the later Neolithic and earlier Bronze Age makes these comparisons valueless. Burial activity on a site prior to the erection of the main structural complex is paralleled at the henge at Cairnpapple (Piggott, S 1948, 76).

\section{PERIOD II (pl 12)}

Ring $\mathbf{A}$ and the ditch and bank appear to be roughly contemporary. The ditch follows the shape of the timber ring quite closely. In common with other class II henges the axis of the two entrances lies away from the centre of the enclosure, resulting in the $\mathrm{N}$ arc of ditch having a flatter curve than the $S$. This imbalance is reflected in the shape of ring A. This would suggest that the ditch was dug while the timbers were upstanding or while some trace of them remained after rotting. The posts could not have been erected after the ditch was dug as the ramps of the pits pointed towards the ditch and terminated in most cases very close to its edge.

At the $\mathrm{N}$ edge of the enclosed area the subsoil surface was over $50 \mathrm{~cm}$ lower than the level of the subsoil under the bank opposite. Part of the difference must have been caused by the relatively protected position of the sub-bank soils but it is possible that some might have been accounted for by the levelling of the $\mathrm{N}$ part of the interior. It is clear that here the bank was built on a slightly higher piece of ground, as beyond it the subsoil surface fell away to the $N$. It is suggested therefore that this small hillock was cut away where it extended into the enclosed area. Nowhere else on site did the bank lie on such an obvious rise. 
Mercer $(1981,149)$ has suggested that the horizontal distance from the uppermost end of the ramp of a post-pit to just short of the opposite side of the pit $(a b)$ is likely to be approximately half the length of the timber to be inserted using the ramp, so that the timber will more or less balance at the end of the ramp. The length of timber would therefore be $2 \times a b$. To assist the determination of the lengths of the timbers used at Balfarg where no ramps survived he examined the ratio of $a b$ to $b y$ (the depth of the pit) at a number of sites. The average ratio proved to be approximately 1:3. At North Mains the average was 1:3.4 (extreme ratios 1:4.8 and 1:2.4).

If the approximate length of timber $=2 \times a b$ at North Mains then the ring was made up of timbers projecting uneven distances above ground; the calculated extremes are $6.52 \mathrm{~m}$ and $2.62 \mathrm{~m}$. As the appearance and function of the structure are still unknown the possibility that the ring was very uneven must be considered. Although the technique of inserting the post by overbalancing into the pit, as suggested by Mercer, is a reasonable method, perhaps the best for the task, there is no proof that it was used, and if used, then universally so. The uneven pit depths might be interpreted as the result of an attempt to raise the top of the timbers of the ring to an even height. Given a supply of timbers differing in length it may have been easier to dig a slightly deeper hole for a long timber than to trim excess length from a timber c $0.5 \mathrm{~m}$ in diameter using stone tools. Shorter timbers would likewise be placed in shallow pits. At North Mains the ramp would therefore have been a roughly constructed adjunct to the pit, designed to ease the erection of the post but not necessarily involving the more precise overbalancing technique suggested by Mercer.

The timber ring was not circular. The $\mathrm{N}$ part of the ring had a flatter curve than the $\mathrm{S}$. Additionally it was built in a series of straight segments (see table 1). The ends of the segments correspond broadly with the ends of groupings of roughly parallel pit ramps. It is likely that these segments represent the division of the circle for administrative purposes during its construction, to ease the laying out; either one team built all the segments one at a time or several teams built one or more each.

The ditch showed no evidence of recutting or segmentation, in the excavated areas. Startin (pers comm) has suggested that two methods of organizing labour for ditch digging can be reflected in the shape of a ditch. Segmentation may indicate the use of a number of different gangs, each digging a pit contiguous with the next. The other method, where a single team dug the whole ditch may be reflected in the arrangement of the ditches at the henge. The NW and SE terminals are larger and more bulbous than the other two; Startin has suggested that this may show that a single pit was dug at those terminals and the remainder of the ditch dug to a face along the remainder of its planned extent, by a single team in each case.

The bank has been so severely eroded that little can be said about its original dimensions. The recovery of post-medieval coins from the mid to upper fill of the ditch suggests that the bank may have survived until recent centuries; it may then have been deliberately slighted to ease tillage. The maximum surviving breadth was $9 \mathrm{~m}$. If this is taken as the original breadth of the bank and if it is assumed that all the material excavated to form the ditch was used in the bank and that no other sources were used (eg turf) then two possible bank dimensions can be suggested. Firstly, if the entrance was the same width as the gap between the ditch terminals, the bank could have been c $2.2 \mathrm{~m}$ high. Secondly, if the entrance was more flared, then a higher bank, c $2.55 \mathrm{~m}$ high, could be postulated. Houlder (1968) has suggested that at Llandegai much of the spoil from the ditch was not used in the bank and this must be considered a possibility at any site.

At the NW terminal a series of features (F9-F21) was originally interpreted as a possible revetment of the bank. However, this was not found elsewhere on the site and it was noted that the feature group stopped immediately to the $\mathbf{N}$ of the modern ditch. It is likely that this sub- 
rectangular setting of posts was a modern fenced enclosure, the fourth side being provided by the drystone dyke which originally ran along the $\mathrm{N}$ side of the ditch.

Few artefacts were found in primary positions in the pits of ring A; only one piece of waste struck stone (SF31) was recovered and the small collection of sherds and fragments of pottery lacked diagnostic feature. Cowie has suggested (supra $\mathrm{p}$ 163) that the pottery was part of a surface scatter associated with earlier use of the area, perhaps only as redeposited midden on agricultural land.

After its construction the Period II monument seems to have been allowed to fall gradually into disrepair. The posts rotted in situ leaving the fill patterns illustrated on figs $6-9$, and the ditch filled naturally, showing no signs of recutting. Some of the fills of the ring A pits were originally intrepreted as showing post removal or other disturbance. Within what would have been the rotted-heartwood areas of the post quantities of oak charcoal were recovered in 19 of the 24 pits. In pit $\mathrm{A} / 7$ a carbonized oak plank was found in a similar position. It is possible that a light wooden structure (or merely a quantity of wood) was burned late in the life of ring A or after it had collapsed. The charcoal from this fire then found its way into the heartwood rot areas of the timbers. Mercer (1981) has suggested some form of fencing between the uprights of the timber rings at Balfarg. It is possible that the burnt remains of a similar arrangement are represented by the charcoal deposits in the ring A pits, especially the oak plank. The relatively small quantities of charcoal in the excavated parts of the ditch fill would not allow a great amount of wood to have been burnt near its edge. The suggested fencing need be no more than a single line of horizontal planks secured to the uprights, though at an unknown height.

\section{Summary of labour organization}

On the henge the limited evidence available might suggest different methods of labour organization in the erection of timber ring A and the construction of the bank and ditch. According to Startin the bulbous nature of the ditch terminals at NW and SE would indicate that the ditch was dug and the bank erected by a single gang, rather than by a number of groups, each responsible for a small segment. In contrast, the erection of the timber ring, which must have taken place before the digging of the ditch, seems to have been organized in sections; the segmentation of the circle, as indicated by ramp angles and straight lines of posts, has been discussed above. It should be borne in mind that the erection of each post need precede the excavation of only the adjacent piece of ditch and that the two parts of the work could have proceeded virtually simultaneously. The segmentation can be interpreted in two ways: firstly that the circle was built by a number of gangs, each responsible for one or more segments; secondly that one gang, perhaps the same group who dug the ditch, built the circle, organizing the digging of the postholes and the collection of wood in convenient units, three to five posts at a time.

The post-pipes preserved in the pits of ring A varied between $0.25 \mathrm{~m}$ and $0.6 \mathrm{~m}$ in diameter. The better preserved pipes tend towards the larger end of the range, suggesting perhaps posts approximately $0.5-0.6 \mathrm{~m}$ in diameter. If, as seems likely, the posts were of oak and given the favourable soils, then trees of age 100-250 years were used. Twenty-four trees of such an age would, in woodland conditions, be found in an area of c $0.5 \mathrm{ha}$; their removal would alter such woodland very little.

\section{Comparanda}

The two opposed entrances of the Period II monument place it in class II as defined by Piggott and Piggott (1939). As at Arbor Low, Cairnpapple, King Arthur's Round Table and Coupland the axis through the two entrances is offset from the centre of the monument, though 
the entrances of the four cited parallels lie at approximately the $\mathrm{N}$ and $\mathrm{S}$ ends of the enclosure, unlike North Mains. At North Mains an entrance to the $S$ would be too close to the steep drop to the Machany Water.

The single timber ring is paralleled at Moncrieffe (Stewart 1974) and Milfield North (Harding 1981) though both are much smaller. The Arminghall setting is horseshoe-shaped (Clark, J G D 1936) as is the small setting at Croft Moraig (Piggott, S \& Simpson 1971). The settings of timbers at Balfarg may have included a single ring (Mercer 1981), though the excavator has postulated a different arrangement of timber heights. In the same report (p 155) the suggestion was made that the stone ring postulated at Cairnpapple (Piggott 1948, 81) may have been a timber setting and therefore, as a 24 post circle, comparable with North Mains.

The replacement of wooden structures by stone has been noted in the vicinity of North Mains at Moncrieffe and Croft Moraig. Likewise it is accepted that stone and timber can be used interchangeably in similar monuments, as a function of the availability of the two materials. Mercer $(1981,154)$ considers that the main timber ring at Balfarg, Fife was a cousin in wood of the recumbent stone circles of the NE of Scotland. It can also be suggested that North Mains and the other henges containing single timber rings can be considered as equivalents to non-recumbent stone circles both free-standing and within henges.

If this is accepted then further parallels for ring A at North Mains can be found. The resemblance of the oval stone (or timber) setting at Cairnpapple has already been mentioned. The ring at Arbor Low, Derbyshire, egg-shaped or oval, bears a strong resemblance to the Cairnpapple arrangement. The class II henge at Brodgar, though much larger than North Mains has the substantial remains of a circle of stones set relatively close to the inner edge of the ditch. There are smaller stone circles within henges at Stenness (class I) (Ritchie, J N G 1976) and at Broomend of Crichie, (class II) (Ritchie, J 1920; Dalrymple 1884).

Burl $(1969,9)$ suggested that stone circles, found only within a relatively small number of henges, were foreign to the original idea of henges. The discovery of what may be timber equivalents to stone circles at Milfield N, Moncrieffe and North Mains, in the last case certainly contemporary with the bank and ditch, indicates that such features might be more common than surface examination and aerial photography would at present suggest. The occurrence of single rings of timber and stone is more common in class II than class I henges, perhaps confirming Burl's conclusion and suggesting that these features in general might appear late in class I sites, if class I sites can still be considered as substantially earlier than class II enclosures. Period II at North Mains can be dated to the later third millennium bc, which accords well with the limited evidence available for class II henges (recently summarized in Burgess 1980, 339). The earlier radiocarbon dates may be from relatively old wood.

The linking, in wood, of the uprights suggested at North Mains and Balfarg might be paralleled in the later linking walling and stones seen at Balbirnie (Ritchie, J N G 1974) and Moncrieffe (Stewart 1974).

If parallels are sought for ring $A$ in structures outside henge enclosures there are few, in timber at least. The embanked circle at Caerloggas in Cornwall (Miles 1973), though of similar size, appears to serve a different function. The timber circles at Bleasdale, dated to the early second millennium bc, also seem to be rather different (Yates \& Jackson 1900; Varley 1938). The frequently found cropmark pit rings noted in recent years during aerial survey in lowland areas may, in some cases, be proved broadly comparable.

Parallels in stone are easier to find, though the North Mains example, with 24 uprights, does not compare well with the body of British stone circles. Burl (1976) lists only 12 examples with more than 20 stones. Only Cairnpapple has 24 . However, circles with a diameter of c 25- 
$27 \mathrm{~m}$ are not rare. Ring $\mathrm{B}$ is compared to timber and stone circles and its position in the sequence is discussed on $\mathrm{p} 188$.

The matter of the possible astronomical significance of henges in general and North Mains in particular has been deliberately avoided. The final resolution of the problem is not far off and the exploration of this aspect of North Mains is best left to those more expert in such matters.

\section{PERIOD III}

\section{Burials}

The burials found within and outside the henge enclosure and in one case dug through the bank have been radiocarbon dated or are of a form, or have artefact associations, to place them in the second millennium bc, after the floruit of the Period II henge. The only exception is burial $\mathrm{F}$, the beaker-accompanied cremation which might have been deposited during the later third millennium, although given the late date for the other beaker-associated activity (infra $\mathrm{p}$ 186) and the relatively 'late' form of the beaker it can be better assigned to the mid-second millennium. They were almost certainly deposited because of the continued importance of the site of the henge enclosure in local life (or death) or because of the possibly more recent erection of ring B, if in fact it did post-date ring A (infra $\mathrm{p}$ 189). It is interesting to note the similarities and differences between the burials at the henge and those dug into the surface of the barrow. These relationships are discussed in the conclusions on $\mathrm{p} 275$.

Burials similar to B and C at the henge, deposited in deep pits, are rare in Scotland. At Broomend of Crichie, a class II henge in Aberdeenshire, a cisted interment (a mixed inhumation and cremation) in a deep stepped pit was found in the centre of the enclosed area (Dalrymple 1884; Ritchie, J 1920). During the excavation of the later prehistoric settlement at Dryburn Bridge two cists set in deep, though not stepped, pits containing double inhumations were investigated (Triscott 1982). At Nether Criggie in Kincardineshire (Kirk \& MacKenzie 1955) a cist was set in a deep pit narrowing towards the bottom. A similar arrangement at Bught Park, Inverness, was described by the same authors and was also noted at Cockburnspath, Berwickshire (a double inhumation) (Hardy 1889). At the henge at Balfarg the central burial was probably originally set in a deep pit (Mercer 1981). A minor structural feature of burial B, the flagging laid around the top edge of the cover slab, can be paralleled exactly on the cist under the Fairy Knowe of Pendreich near Bridge of Allan (Alexander 1868). A similar arrangement was noted at Wester Wooden, Roxburghshire (Winning 1886) and in one of the two cists at Dryburn Bridge. At North Mains the flagging served to complete the sealing of the cist in part of the capstone's circumference; there was no functional reason for the complete circuit of flagging. Pavements over burial features as at the top of the pit of burial B have been described in NW England (Burgess 1980, 317). Once again at Dryburn a pavement was laid immediately over the capstone of one of the deep cists, not, however, near the surface as at North Mains. At Dryburn the pavement had a beaker laid on it and therefore presumably served a function in the burial ritual; no evidence for such a role was found at North Mains though it was, perhaps, the likeliest reason for the construction of the paving.

Though resembling each other in some ways there are differences between burials B and C, apart from the contents of the cists. $C$ is set in a cist rather neater in execution than $B$. Whereas in $\mathrm{B}$ an attempt had been made to prevent the ingress of soil, with some success, in $\mathrm{C}$ it was clear that the pit had been backfilled to a level well above the top of the cist prior to the placing of the cover slab, an activity paralleled in some food-vessel-accompanied burials (McAdam 1974, 43).

Burial D was an example of the more normal type of food-vessel-associated cist, formed of rough slabbing in a shallow pit, although severely disturbed. The presumed inhumation was 
accompanied not only by a food vessel but by a small deposit of cremated bone, possibly not human. Similar mixed deposits are not uncommon and have been found, for example, in the central burial at Broomend of Crichie (Dalrymple 1884; Ritchie, J 1920) and at Dalgety Bay, Fife (Watkins 1982, 70). Cist 1 at Dalgety contained an inhumation and a cremated human accompanied by a food vessel. In Grave 1 the inhumation was accompanied by three cremations, one possibly not human, a food vessel, and a battle axe of Roe's type A, all within a wooden or leather coffin. Further examples of single inhumations accompanied by one or more cremated humans were found in two cists at Dunchraigaig, Argyll, one with a multiple cremation, the other a single cremation with a food vessel (Greenwell 1866); at Balnabraid, (cist g), Argyll accompanied by a food vessel (Galloway 1920); at Camelon, Stirlingshire, also with a food vessel (Buchanan \& Callander 1923); at Almondbank, Perthshire with a food vessel (Stewart 1973).

Burial $\mathrm{E}$, an inhumation, surviving only as fragments of bone, had apparently been placed in an uncisted grave with a food vessel of which only a few sherds were found. Whether more sherds or indeed a whole food vessel were originally deposited and have since been destroyed or scattered is not known. It is conceivable that the pit, dug through the bank of the henge, was not a burial pit but had been filled with bone and sherds from a disturbed grave. Interments deposited in pits without a cist, but possibly on occasion with wooden or leather coffins, are rare in Scotland, relative to cisted burials. As with deep set burials like $B$ and $C$, the difference may be the result of the ease with which shallow, cisted burials can be found by ploughing, quarrying and other developments (Stevenson, J B 1975; Barclay 1982). Excavations like Dalgety Bay and North Mains may show something of the true proportion of uncisted graves to cists (Watkins 1982).

Burial $\mathbf{F}$, as a beaker-accompanied cremation, is a definite rarity in Britain. D L Clarke $(1970,453)$ lists 34 examples. He has suggested that the antiquarian reports of many of the discoveries are unreliable and that only 14 can therefore be considered 'probable' examples. Only two are in Scotland; at Buckie, Banffshire (Young 1895) and at Stoneykirk, Wigtown with a beaker/food vessel hybrid (Mann 1902). He does not, however, include the certain example from Kilmarie, Skye (Callander 1928). Cowie (supra) has suggested a date for the North Mains vessel in the mid second millennium bc, which would be consistent with the radiocarbon assay for F33. The apparently unnecessary covering of the capstone with further smaller slabs cannot be satisfactorily explained; perhaps a role within the burial rite can be suggested for this feature, as in burial B. Mercer $(1981,164)$ has noted the rarity of beaker burials in henges in England and has listed the known British examples.

Burial G, a cremation, was similar in some ways to B and C; it was deposited in a deep, slightly stepped pit, though without a cist. The neatly defined edge and the shape of the deposit suggested that it may have been placed there in a bag. A possible parallel for the boulder-filled pit is to be found at the henge at Yeavering (Harding, A 1981).

Burials $\mathrm{H}, \mathrm{J}$ and $\mathrm{K}$ were unremarkable; the interment of cremated humans in urns is hardly an uncommon rite, and can be paralleled at henges; eg at Moncrieffe (Stewart 1974). However, burial $\mathbf{J}$ was multiple and the urn was of an unusual form, perhaps combining more than one urn tradition (Cowie's report infra $\mathrm{p} 256$ ). The proximity of burials $\mathrm{H}$ and $\mathrm{J}$ to each other might suggest that they were buried at approximately the same time; one may have preceded the other, attracting the second burial because its position was marked. F25, immediately by burial $\mathrm{K}$, may conceivably show the position of just such a grave marker.

Burials $\mathrm{L}$ and $\mathrm{M}$, as discussed above, may be contemporary with burial $\mathrm{A}$, or may reflect a further tradition present in Period III. F2 and F51 in the Miscellaneous Features section might both be interpreted as further burials of Period III; F51 resembles the deep pits used for burials $B, C$ and $\mathbf{G}$. 
The palaeopathological evidence was limited. Early signs of osteoarthritis were noted in the older individual in burial J. The lower part of the body was not represented in the identifiable fragments from burial M. Powers has commented that this is unusual. The low weight of the remains might suggest that only half of the body was burned. In burial $\mathrm{M}$ only one bone fragment was duplicated. However, Powers (p 174) has suggested that the deposit consisted of only one individual's remains and that the duplicated malar fragment was an accidental inclusion. This might indicate that the cremation was carried out on or near the site of an earlier pyre and that a fragment of another burial was mixed in by accident. The only surviving inhumed skeleton (burial B) showed no signs of disease and had well preserved teeth.

The pottery finds have been discussed by Cowie ( $p$ 248). The very close resemblance of SF17, the food vessel from burial B, to the example from Cowdenhill, Bo'ness (fig 29) has been noted. Cowie has mentioned the possibility that the two pots were the work of the same hand. It is hoped that the further work will test this suggestion; discussion of the implications will be reserved until it is completed. The vessel is also noteworthy for the results of the pollen analysis carried out by Bohncke on the deposits found within it. It is certain that the vessel had contained a cereal-based material flavoured with meadowsweet; a gruel of some sort or perhaps even a fermented drink. The results of the analysis of scrapings from the Ashgrove beaker (Dickson 1978), which had contained a lime-based honey or mead flavoured with Meadowsweet, are comparable. The analyses claimed to identify the presence of cereals in the Knappers and Mount Vernon vessels (Davidson 1935) are of doubtful value. The blackening of the cist cover slabs over the vessels at Kemnay, Dyce (Stuart 1857) was suggested by the excavator to be the result of burning the contents in the pots. That the beaker phenomenon is related to the possible alcoholic nature of the contents of the pots has been discussed most recently by Burgess $(1980,63)$ who has compared the spread of the pottery and the rest of the 'package', with the rapid expansion of the Peyote Cult of Central and N America. Given our lack of knowledge of the precise use of the pots, Burgess's objections to food vessels also being used to hold liquids because of the form of the rim cannot be sustained. Hawkes (1981) has on the contrary suggested that the slightly overhanging rim might hold back the sediment or porridge and allow the 'boozy juice' to be sucked off.

\section{Domestic or ritual activity}

Such activity was represented only in the debris found in one pit (F33) and in some of the hollows formed in the tops of the ring $\mathrm{A}$ pits. The beaker associations are clear; sherds were found in the hollows of $\mathrm{A} / 7$ and $\mathrm{A} / 8$ and a burnt, broken barbed and tanged point was recovered from the fill of F33. However, when the burnt animal bones are considered it is unclear whether the evidence is of cooking or of the cremation of the animals. The cooking itself might be related to a continued ritual use of the enclosure or to a short occupation by a group doing something more prosaic. However, given the use of the site for burial in the mid second millennium bc, and the occurrence of burial close by in the late second/early first millennium bc, any use of the site in approximately the 15 th century bc is likely to have been a continuation of the ceremonial function of the enclosure. It is suggested below ( $p$ 189) that ring B may have postdated ring A and may therefore have been a refurbishing of the monument. The active consideration of the area as a ritual site may thus have been prolonged. Barnetson's examination of the animal bones has suggested that pig was definitely present in the assemblage with the addition of other small and large artiodactyla (ovicaprids and bovids perhaps), though the nature of the remains has made precise identification very difficult. The lithic report (supra $\mathrm{p} 167$ ) suggests that no primary knapping took place on the site in this period but that secondary work, such as re-edging and sharpening, did. A variety of wood species was used in the cooking or burning. 
Cowie has noted the general similarities between the beaker sherds found in the ring A pits and the complete vessel found with burial F, and that all are likely to belong to the mid second millennium bc, a date consistent with the radiocarbon assay of charcoal associated with the barbed and tanged arrowhead found in F33.

It is clear that in this period substantial parts of the enclosed area were covered with charcoal (of mixed variety) and burnt animal bone, probably of domesticated species. It is possible that ring $B$, the smaller timber ring, was erected after Period II and therefore at some time within the portmanteau Period III. However, the lack of burnt material in the ring B pits suggests that they were not dug at a time when charcoal covered extensive areas of the enclosure (infra $\mathrm{p}$ 189).

Material that may be interpreted as occupation debris has been found in the ditches at the henge at Gorsey Bigbury (ApSimon 1949) and Durrington Walls (Wainwright \& Longworth 1971, 20). Beaker sherds in the secondary silts of a number of henge ditches (Catherall 1974, 40) may also show continued interest in the sites but very different activities might be represented by the scatters at individual sites.

\section{PERIOD IV}

The four pits to the $\mathrm{N}$ of the enclosure (F3-6) and the secondary activities within them seem to reflect some continuing tradition of the use of the area for burial, though the avoidance of the enclosure, which must still have been visible, may be significant. The date of the primary pits beneath remains obscure, apart from the terminus ante quem provided by the secondary activity. The cremation and burial in situ took place in hollows, apparently formed naturally, in the tops of the pits. However, a considerable time may have elapsed between the filling of the pits, the subsequent settling of the fills to form the hollows and the use of the hollows for cremation.

No artefacts or organic deposits were found in the primary pits or in the recuts which were found in F4 and F6. Both recuts had apparently been made before the primary pits were completely backfilled. The recutting might make the interpretation of the pits as burial features rather difficult to sustain unless the recuts showed multiple deposition. The later activity, as represented by cremated bone and charcoal, was noted in all four hollows though later disturbance had affected them unequally. In F3 charcoal-stained soil, burnt bone (possibly not human) and a rather disturbed stone setting similar to that found in F6, lay on a fire-reddened surface. F5 and F6 also produced clear evidence of burning. In the former the bone was of unidentifiable species, but in F6 a burial (burial O), certainly human, was contained in a well-formed stone setting. The evidence for burning in situ in F4 was not conclusive; the burnt bone and charcoal may therefore have originated in one of the other pits.

It is likely that the hollows on the top of the fills of F3, F5 and F6 are the sites of the cremation of human remains and their burial on the pyres, in F3 and F6 with the elaboration of a stone setting. The radiocarbon series places this period of activity firmly in the late second/early first millennium bc, comparable with the date of charcoal from the pyre burials at Claggan in Argyll (Ritchie et al 1975). Cairn 1 at the site was a $5 \mathrm{~m}$ diameter stone annulus surrounding an area of burning, interpreted by Ritchie as a pyre. Within this enclosed area was a stone setting c $2 \mathrm{~m}$ in external diameter (not $4 \mathrm{~m}$ as stated in the report) surrounding a space 1.25 by $1 \mathrm{~m}$ in which were deposited the cremated remains of a single human. This inner setting resembles those at North Mains in F3 and F6 in which the bulk of the remains were placed. At Claggan the pyre and stone setting were finally covered. At North Mains the depth of the modern ploughsoil represents the minimum loss to erosion and disturbance over the burials. It is possible that these features were originally covered by a low mound but it is almost certain that the hollows, which must have been at least c $0.4-0.5 \mathrm{~m}$ deep below the contemporary land surface, were filled up at 
least to the level of the modern surface after the depositions took place. To the $\mathrm{N}$ of the excavated area, crop-marks indicate that more pits similar to F3-6 may exist.

The discoveries at Claggan and North Mains show that the rite of pyre burial, found occasionally earlier in the second millennium bc, survived into the later part of the Bronze Age in Scotland. The rite has been found in northern England, dated to approximately the 1st century BC (Alnham; Jobey \& Tait 1966). It has also been found under Irish Iron Age barrows (Cush, Co Limerick; O'Riordain 1940).

Burl $(1972,44)$ has suggested that the sequence of development of some ring-cairns associated with recumbent stone circles is as follows; firstly the erection of the circle, secondly a cremation pyre and lastly the building of the ring-cairn round the scraped-together burnt debris. This may well have been an earlier reflection of the tradition found at the end of the millennium at North Mains. The relationships between recumbent stone circles and the Balfarg henge, and between ring-cairns and the North Mains barrow are discussed below (p 273).

The rite of cremation and in situ burial is known under the round and long barrows of the $\mathrm{N}$ English Neolithic but only rarely later. There is a little evidence in the Clava and NE Scottish ring-cairns that pyre burial may have been part of the rite in the late third and earlier second millennia (according to the present dating of the sites). Further excavation in the ring-cairns of the Clava group and the NE of Scotland may clarify this. Kilbride-Jones $(1935,219)$ suggested pyre burial under 'Ring Cairn 2' at Cullerlie, Aberdeenshire. He also noted the existence of a pyre site in the centre of the enclosed cemetery at Loanhead $(1936,282)$. Although this was interpreted as the pyre for the deposits interred on the site it is possible that the cremated bone found in the burnt area represented a single pyre burial, and not an accumulation of fragments from many cremations.

\section{PERIOD V}

The burial in LG3, the bone fragments in some of the similar pits and the common orientation suggest that all 13 of these features (LG1-13) are graves of a similar date. The change in orientation towards the $S$ of the group might hint that the criteria for the direction of burial changed; they may have been buried at a time of year when the sun rose further to the $\mathrm{N}$ or a landmark on which the graves were aligned may have lain further to the $\mathrm{N}$ if viewed from the $\mathrm{S}$ of the enclosure.

The radiocarbon date from the burial and the orientation of the graves make it most likely that the cemetery was a Christian one. The enclosure of such cemeteries by circular or oval boundaries (walls, ditches and banks) is common and the use of pre-existing enclosures is not unknown. Thomas $(1971,53)$ lists examples of Christian burial imposed on pre-Christian cemeteries in N Britain and elsewhere. The long graves at Cairnpapple may also be of the early Christian period though the excavator suggested an earlier date (Piggott, S 1948, 100). It is possible that, as in other cases, the cemetery was placed in the enclosure not merely to use the existing boundary but to 'Christianize' a pagan site. Examples of this are common; for example the inclusion of such monuments as the Midmar Kirk (Aberdeenshire) recumbent stone circle (Coles, F R 1900) and the Rudston monolith within churchyards and the building of a church in the centre of the henge at Knowlton Central Circle, Dorset.

MISCELLANEOUS FEATURES

\section{Ring $B$}

This elliptical setting of 17 , or possibly 18 pits, probably the remnants of a post ring, is not stratigraphically related to other features on the site. However, there is some evidence which may 
help locate it in the sequence at North Mains. No charcoal was found in any of the post-pits, but a few fragments of cremated bone were retrieved from one pit; however, Ring B was therefore probably constructed at a time when little charcoal lay on the ground. Therefore the pits were probably not dug or disturbed during, or in the aftermath of, one of the episodes of burning in late Period II or the ritual or domestic episode of Period III; a certain amount of charcoal would certainly have been incorporated in some of the fills. However, the ring could have been erected some time after the Period II burning episode, ie in Period III prior to the deposition of the later burnt material.

As with ring $A$, the best parallels may lie in the stone circles. If so then Burl's comments (Burl 1976, 46) on the sequence of shapes and sizes of stone circles may provide a further clue to the chronological sequence of the two rings. He notes the tendency of oval rings to post-date circular settings, and of larger rings to be replaced by smaller. Thus ring B is more likely to be the successor of the Period II monument than vice versa, despite the eccentric position of ring $\mathbf{B}$ within the enclosure formed by the ditch and bank. The location of ring B completely within the ditch of Period II suggests either that its site was obvious when ring A was erected or that B was placed within the pre-existing enclosure.

Ring B falls into the average size range of the less numerous sub-group of 'large oval stone circles' (ring B: 22.5 by $18.5 \mathrm{~m}$; average 21.9 by $20.7 \mathrm{~m}$ (Burl 1976, 50)). The circles at Easthill, Holm of Daltalloch and Loch Roan, all Wigtownshire, are the nearest in size to ring B at North Mains; local circles are much smaller.

The remaining features may in some cases be assigned tentatively to some of the identified periods. F2 may be a burial pit of Period III as may F51. F25 is almost certainly a posthole, possibly associated with burial $J$ as a marker. The charcoal-stained soil in F23, F29, F37, F38, F40, F42, F43, F45 and F50 may show a relationship to the two phases of charcoal spreading on the site (at the end of II; during III) though it does not prove one. F37 and F38 may form a pair of postholes at the $\mathrm{N}$ edge of the enclosure. F40-43, F45 and F54 may also be identified as postholes.

\section{The barrow}

\section{INTRODUCTION}

The North Mains mound lay on the same plateau as the henge and some $200 \mathrm{~m}$ to the WSW (pl 13). Despite its great size before excavation (approximately $40 \mathrm{~m}$ in diameter and $5.5 \mathrm{~m}$ high) it was not recorded by the Ordnance Survey until May 1967 and was not scheduled as an ancient monument.

\section{PREVIOUS WORK ON THE SITE}

There had been three episodes of disturbance prior to the excavation. The first, of which no more than the information gleaned from the current excavation is available, appeared to have taken place in relatively recent times. A deep, narrow shaft had been dug from the top of the mound to within $1 \mathrm{~m}$ of the old ground surface. Near its bottom was found a rusted iron shovel with part of its handle still intact. The nature of the boulder core of the mound made the differentiation of disturbed and undisturbed material very difficult. However, the edges of the central stone feature were not disturbed. The shaft was almost completely backfilled using the excavated boulders with an admixture of soil in the upper part. In 1957 a further excavation was undertaken by Sir William Murray and Mr William Haxton. The only record of this work 
survives in a site notebook of Mr J Harrison Maxwell (now deposited in the National Monuments Record of Scotland, manuscript no 26); my thanks are due to J B Stevenson who drew my attention to this reference. When excavating in the area in 1958 Harrison Maxwell visited the mound on the 27th August:

'6.30 pm-8 pm William, Charles, Nicholas and I to see Balgreenie Cairn, North Mains, Strathallan. William and Young Huckston [Haxton] had dug into [it] last summer. They used a tractor with caterpillar wheels and a wire hawser and so lifted out a huge cover slab 5 feet by 6 feet from centre of Cairn. Its under surface had 20-30 cup-markings.'

The cupmarked stone was left next to the $1.5 \mathrm{~m}$ deep crater formed by Murray and Haxton; it had certainly been moved from its original position during the first, undated disturbance. The third and last disturbance occurred between the excavator's preliminary visit to the site and the commencement of work in 1978. A pit was dug into the WNW flank of the mound deep enough to penetrate the old land surface. Mr Haxton, now of Inverurie, kindly confirmed the description of the 1957 excavation and made the excavator realize that the earlier, undated, excavation was indeed a separate episode.

The mound had also suffered severe disturbance from tree roots and the burrowing of rabbits. Many mature trees, mostly beech, planted in the mid to late 19th century, had grown on the mound until felled in 1976. The removal of the stumps caused great problems during the excavation as the largest weighed in excess of 3 tons. Root disturbance was concentrated in the upper $1.5 \mathrm{~m}$ but extended to and through the old land surface, even in the mid part of the mound. Animal disturbance was concentrated on the $\mathrm{E}$ side of the mound, the NE quadrant suffering worst of all; the layer below the stone capping was honeycombed with tunnels. The most serious effect of the root and rabbit disturbance was to destroy the precise contexts of the secondary burials near the surface of the mound.

Examination of the crater of the 1957 excavation suggested to the Ordnance Survey that the mound was built entirely of stone, an interpretation supported by the great number of stones showing through the turf. The 1978 disturbance in the NW quadrant revealed a profile which was stone-free except for an outer skin. After the turf cover was removed a stone capping was revealed (pl 15) covering a mound made of gravel, sand, silt and turf, though with substantial stone elements, largely restricted to lenses within the mound and to a central stone core into which the first, undated, and second (1957) disturbances had penetrated.

Around the mound there was no trace of a ditch except in the NW sector to the S of a modern drystone dyke, where a hollow, lined on its outer side by tree stumps, was visible. It seems likely that the tree line there protected the ditch from encroachment by modern agricultural activity.

The site was laid out in four quadrants, the dividing baulks aligned N, S, E and W. Extensions were made to the NW, NNE and $S$ to allow the examination of the putative ditch. The excavation lasted from June 1978 to July 1979, with a break of two and a half months ai the beginning of 1979. After the removal of the turf to reveal the stone capping, the investigation of the mound was commenced with the excavation of spits of material in the quadrants at its peak. When approximately $2 \mathrm{~m}$ had been removed by hand, the pattern of internal divisions had become clear. Due to the limited time available for the examination of the mound it was decided that much of the site would have to be excavated in level $0.25 \mathrm{~m}$ spits by machine, a plan of the visible internal divisions being made at every $0.5 \mathrm{~m}$. A similar form of recording was used in the SE quadrant where, apart from one $0.25 \mathrm{~m}$ spit near the bottom, all the work was done by hand. 
In the first season the site was excavated to the level of the old land surface; the central area was cleared, leaving only parts of the cross-baulks distant from the centre; the ditch at the $S$ and at the NW were excavated. In the second season the features cut through and covered by the old land surface were investigated, the NW ditch section was completed and a further section was cut to the NNE of the site.

\section{DESCRIPTION}

The following sequence was recovered:

i Period I The digging of three features, sealed by the old land surface.

ii Period II The use of the old land surface prior to the construction of mound-associated features.

iii Period III The construction of the mound; subdivided into six phases $(\mathrm{A}-\mathrm{F})$ :

(A) the wooden substructure (further subdivided in discussion, $\mathrm{p} 232$ )

(B) the construction of the ring-bank; quarry ditch begun

(C) the construction of most of the remainder of the mound; quarry ditch completed

(D) activity in a hollow left in the top of the mound at the end of (C)

(E) the filling of the hollow

(F) mound covered with stone capping

Ten burials were dug into the surface of the mound, some possibly during its construction, or in a slightly later period. The cupmarked slab may date from Period III/C or later.

\section{PERIOD I}

The earliest recorded activity on the site was represented by a series of features sealed by the old land surface (OLS). Any occupation surface associated with these pits had been destroyed by the subsequent agricultural use of the fossil topsoil (Period II). Thirty-three features were noted and investigated in the excavated area (fig 38). Sixteen were certainly of natural origin (root and animal activity), six were possibly of human origin and eight probably of human origin. The last eight are fully illustrated (fig 39) in the report (F4 to F11). A further three, F1, F2 and F3, were without doubt deliberately dug pits; F1 was a posthole, F2 was a shallow pit dug into the top of $F 1$, and F3 may have been a second posthole, less substantial than F1.

F1 (fig 39) measured $0.4 \mathrm{~m}$ in diameter and $0.7 \mathrm{~m}$ deep. It appeared to have held a post packed in position with stones. The section line was not ideally placed to record the post-pipe but it is likely that the post rotted in situ. A shallow pit (F2) was subsequently cut into the top of F1 and filled with charcoalstained soil and a clean loam. In the latter, two sherds of pottery, apparently from the same vessel, were recovered (SF13). F3 (fig 39) was a small pit with a uniform loam fill and measured $0.2 \mathrm{~m}$ in diameter and $0.2 \mathrm{~m}$ deep. It may have held a slight post.

The remainder of the features had largely uniform loam fills with random stone inclusions, with the exceptions of F4 and F5. Some fragments of charcoal were recovered from the fill of F11 but no material suitable for dating, or artefacts were found in these pits. As noted above, the contexts of material found in the fossil topsoil or in the worm activity zone below it are not secure. Sherds from vessels were found both in natural feature $A$ and the fossil topsoil above it (SF1, 2, 5-9, 11, 12).

\section{PERIOD II}

Subsequent to the digging and use of the Period I features the fossil topsoil was used for agriculture. No ard-marks or any other direct evidence for the use of such an implement was found on the site but most of the $\mathrm{N}$ part of the old land surface (OLS) covered by the mound had been dug into parallel ridges and furrows (pl 13b). The ridges were a maximum of $0.15 \mathrm{~m}$ high and measured 1.8-2 $\mathrm{m}$ from crest to crest. The ground surface buried under the ridges was found by the microscopic examination of a soil 


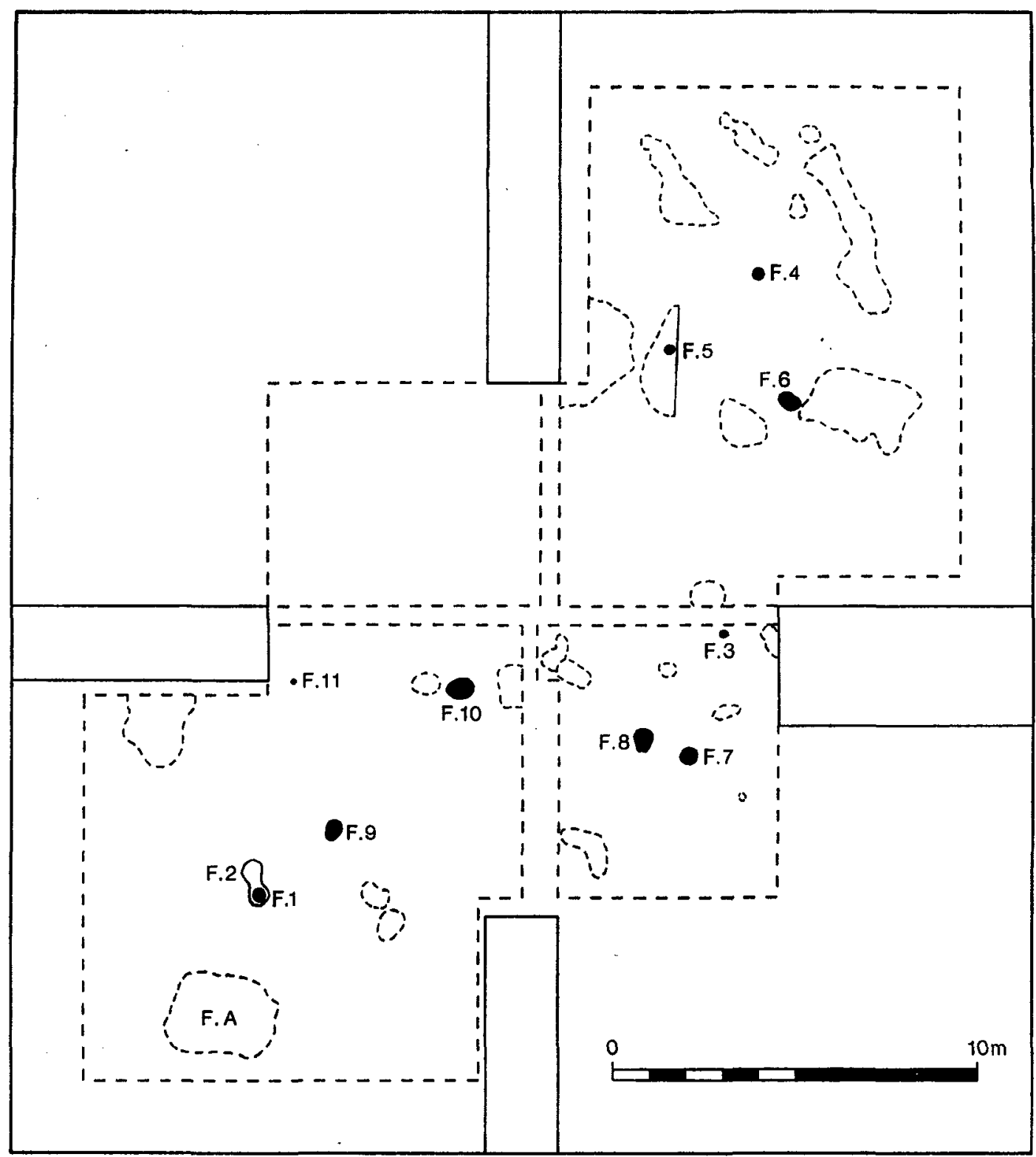

FIg 38 Barrow: natural and non-natural features sealed under the old land surface (OLS); the edges of natural features are shown by dashed lines

section. It was not visible on macroscopic examination on site. Within the fossil topsoil quantities of pottery and chipped stone implements and waste were found. The periods of their origin are unclear. They may have been deposited immediately prior to the erection of the mound or may have lain in the soil for some length of time. At the $\mathbf{N}$ edge of the mound a charcoal deposit was found at the interface of the OLS and the redeposited turf dumped on it. The charcoal may have been deposited in Periods I, II or the early part of III; it was dated to $1855 \mathrm{bc} \pm 100$ (GU-1134). The OLS near the centre of the barrow was a hard grey gleyed soil. Towards the edge, where root and animal disturbance had a greater effect, it became increasingly red/brown (see soil report p 260). 


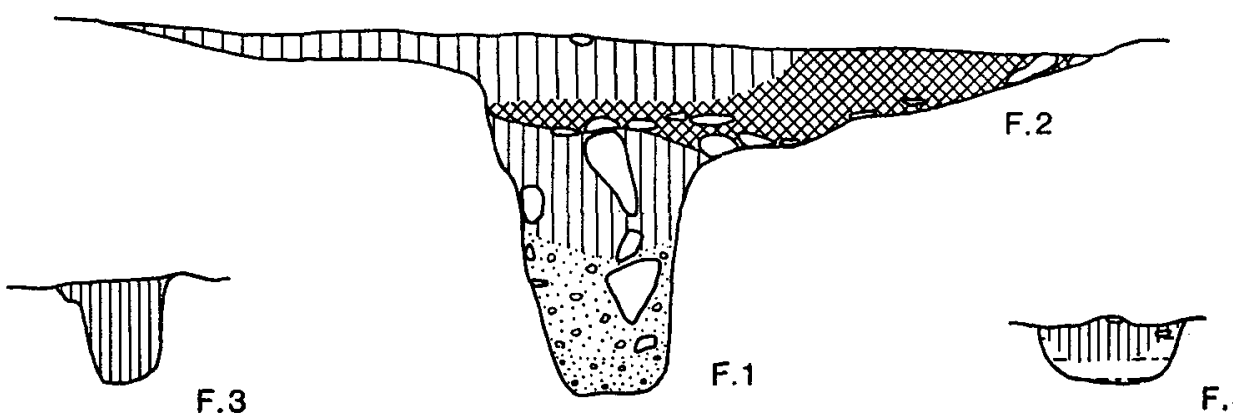

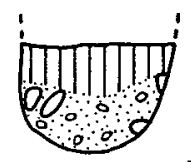

F.5

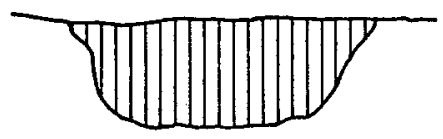

F. 6

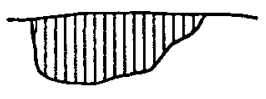

F. 8

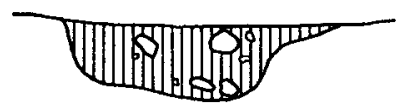

F.9

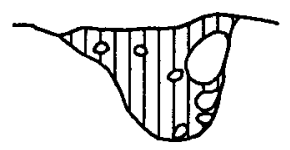

F.7
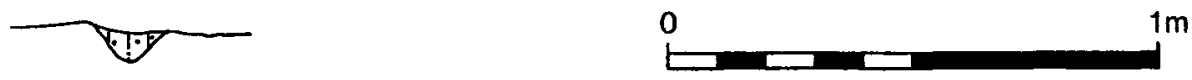

F. 11

Fig 39 Barrow: sections of non-natural features sealed under the OLS

\section{PERIOD III}

This period covers the construction and use of the sub-mound structures, the various episodes in the erection of the mound and the possibly later additions to the structure. Little comment is made on the interpretation of their likely sequence; a full discussion is to be found on $\mathbf{p} 232$.

\section{Period III/phase A: the sub-mound features}

Close to what was to become the centre of the mound a small posthole (F12) cut from the OLS was found. It measured $0.15 \mathrm{~m}$ in diameter and $0.57 \mathrm{~m}$ deep. $3.4 \mathrm{~m}$ to the $E$ a second posthole was found, which measured $0.15 \mathrm{~m}$ deep and $0.2 \mathrm{~m}$ in diameter (F13). From F12 three grooves, up to $0.05 \mathrm{~m}$ deep and $0.1 \mathrm{~m}$ broad, ran to the $\mathrm{W}(\mathrm{F} 17)$ for $5 \mathrm{~m}$, to the $\mathrm{S}$ (F16) to the edge of the excavated area and to the $E$ (F14) to just short of posthole F13. To the E of F13 the groove restarted (F15), and continued to the E to the edge of a disturbance associated with a later phase of construction where its line was lost.

Centred very roughly on F12 was a subcircular arrangement of post- and stakeholes (fig 41) between 6.8 and $7.6 \mathrm{~m}$ in diameter. At the NE was a complex arrangement of stakes and grooves. The evidence of impressions surviving in the mound material suggested that the posts of the $S$ half stood up to $2.4 \mathrm{~m}$ tall 


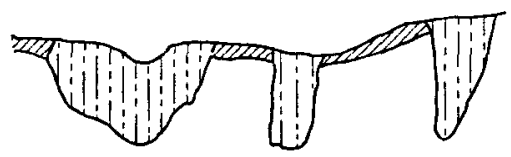

2000009
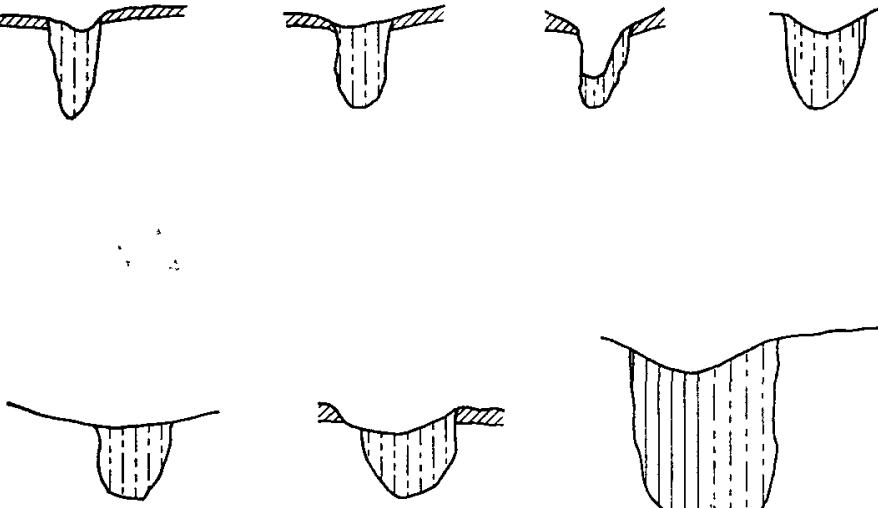

a

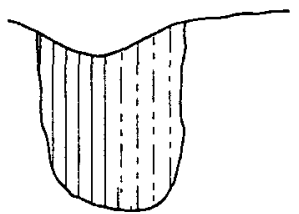

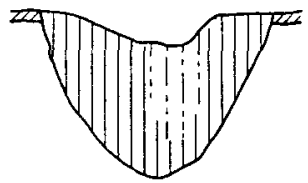
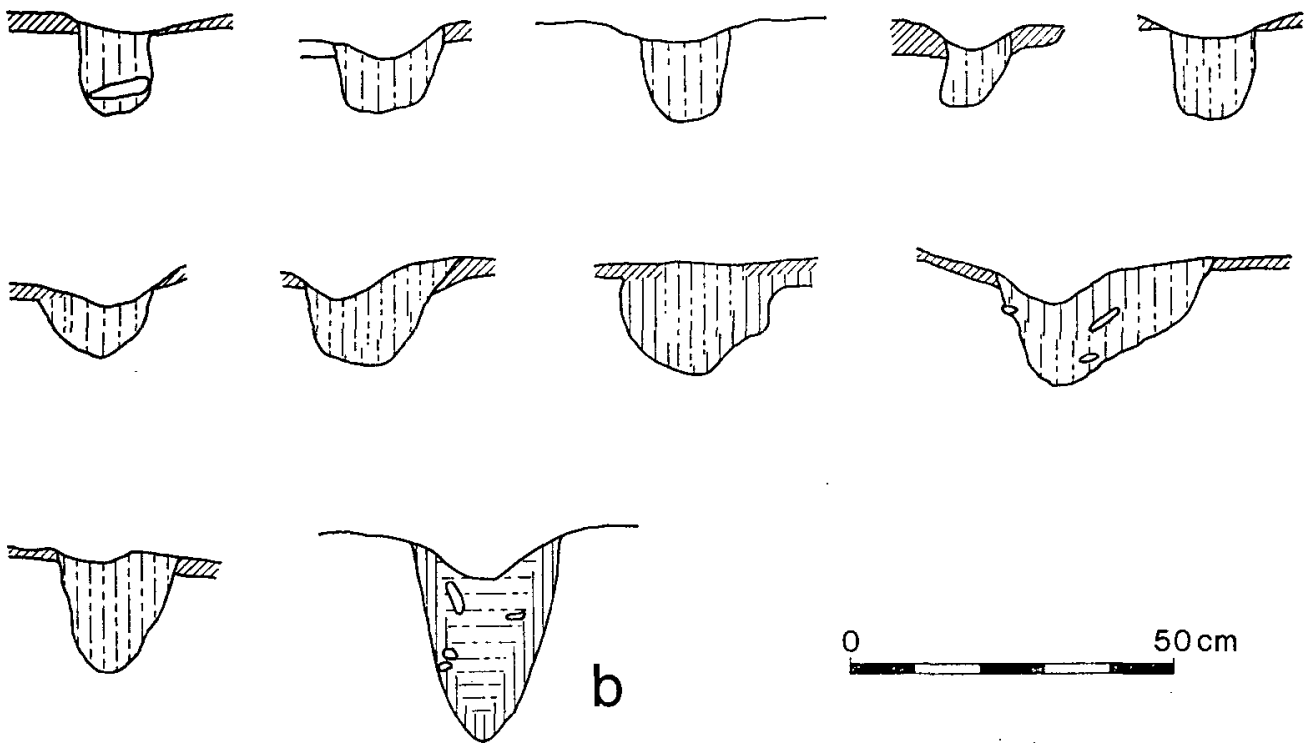

FIG 42 Barrow: sections of stake- and postholes of the central enclosure ( $\mathrm{a}-\mathrm{NW}$ quadrant; $b-\mathrm{SW}$ quadrant)

from the ground surface and were c $0.1-0.25 \mathrm{~m}$ in diameter. The $\mathrm{N}$ half of the enclosure was built using thin wands, in contrast to the substantial posts in the $S$ part. The post pits in the $S$ half were between 0.2 and $0.6 \mathrm{~m}$ in diameter and between 0.2 and $0.7 \mathrm{~m}$ in depth. In the $\mathrm{N}$ half the stakeholes varied between 0.1 and $c 0.25 \mathrm{~m}$ in diameter and between 0.1 and $0.3 \mathrm{~m}$ in depth (fig 42). No mixing occurred at the edge of the enclosure between soils dumped inside and outside it in Period III, suggesting that the uprights were linked by fencing.

On the old land surface in the $S$ half of the enclosure and to a limited extent outside it on the $S$ and 


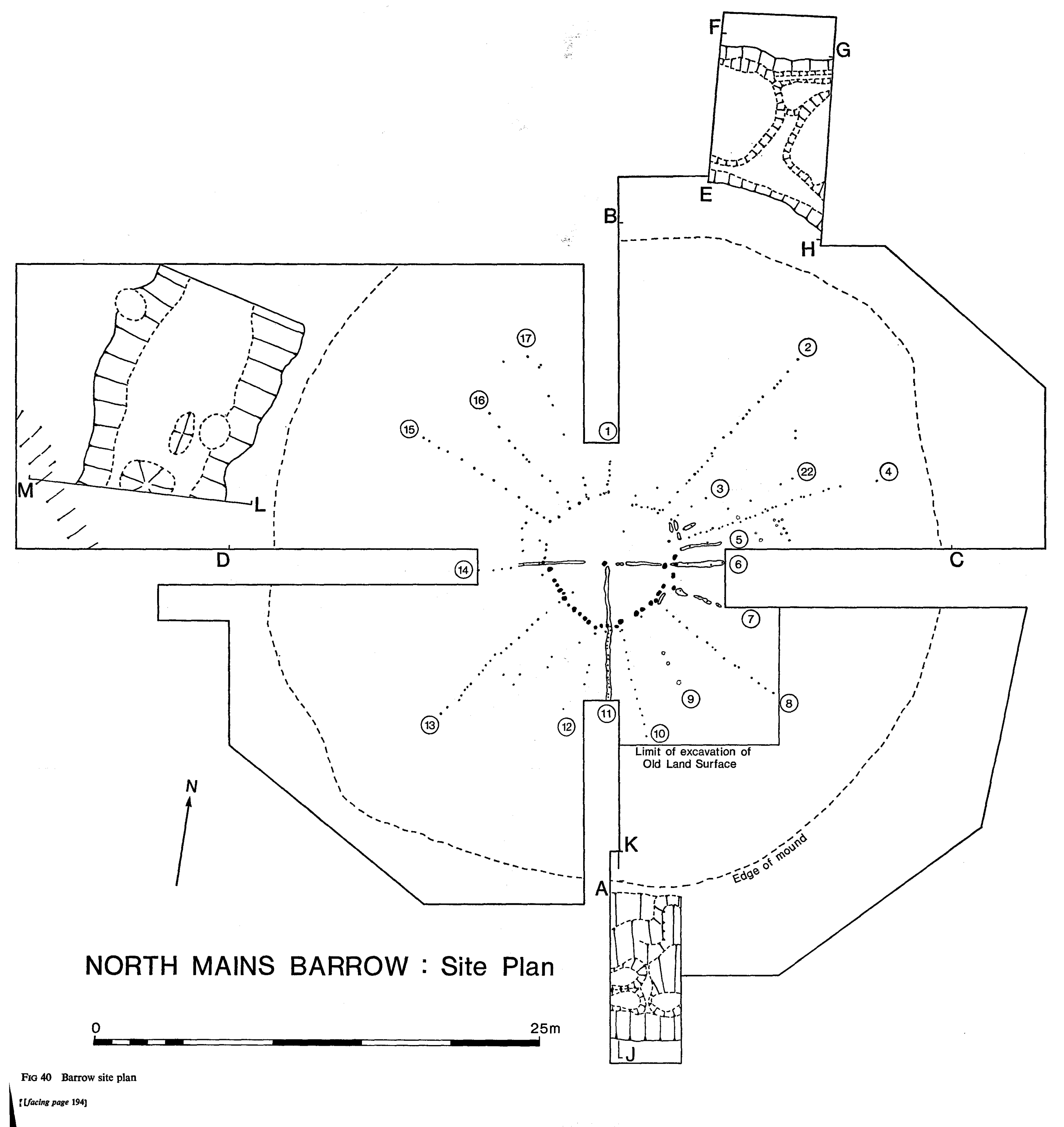




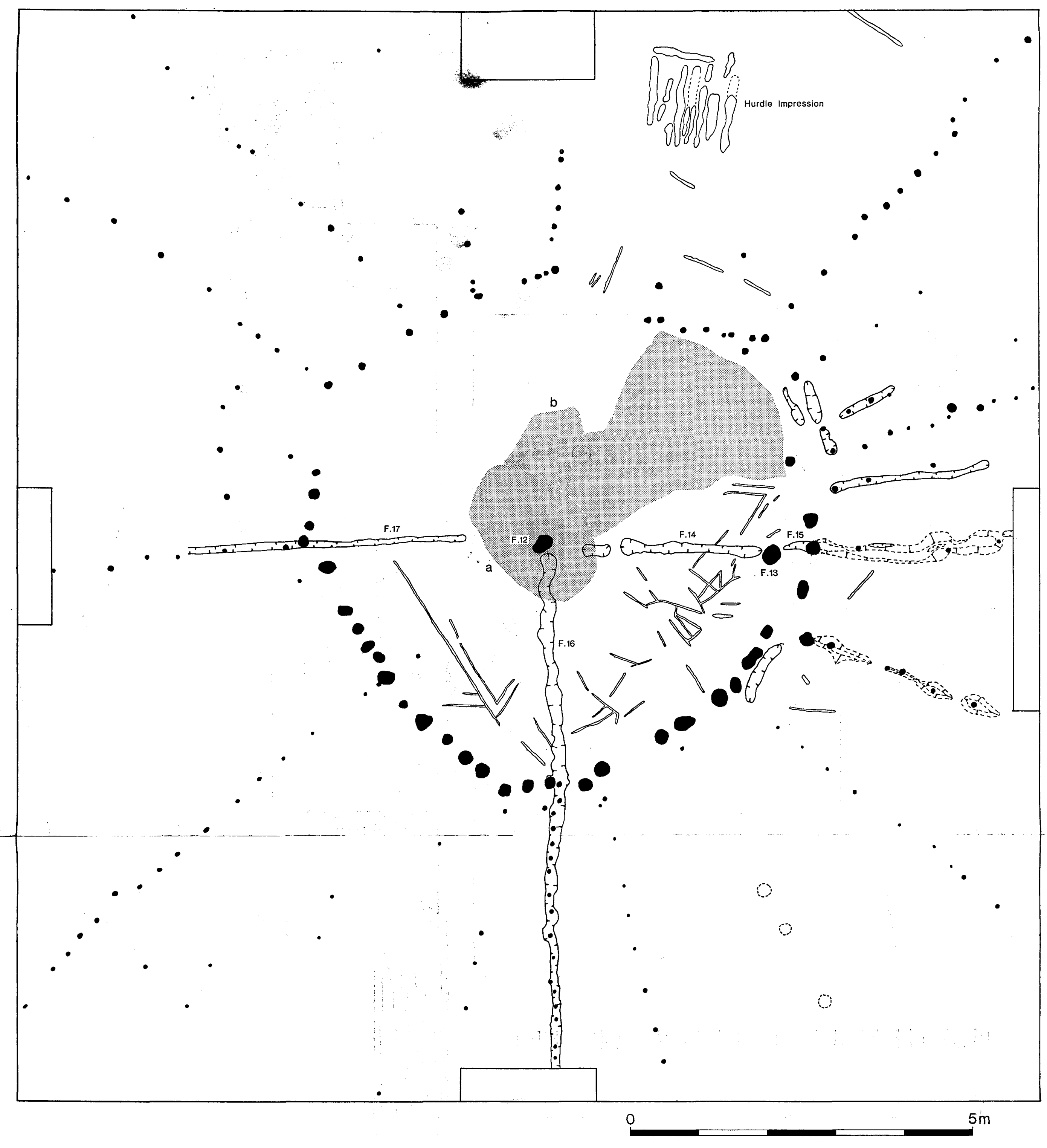

FIG 41 Barrow central area: $a$ - dark grey area; $b$ - soft red-brown area 
E sides were found thin ridges of varying length. Only the most substantial could be planned; the photograph ( $\mathrm{pl} \mathrm{14)}$ provides a better impression of their appearance and density. Within the enclosure three distinct soil types were noted (fig 41). Most of the enclosed area was similar in character to the rest of the OLS under the main mass of the barrow, a hard, silvery grey material. In the central part a distinctly darker area measuring a maximum of 2.2 by $1.4 \mathrm{~m}$ was noted (fig 41 ). Leading from this towards the NE was a broad band of red-brown soil much softer than the surrounding material.

Samples of soil for phosphate analysis were taken at $1 \mathrm{~m}$ intervals (see report on p 269 and fig 65). The results, briefly summarized, show a high concentration 1-2 $\mathrm{m}$ to the $\mathrm{E}$ of the central post; medium concentrations on the dark area at the centre and in the adjoining part of the broad red-brown band and in small parts of the central area. Most of the rest of the enclosure and the remainder of the sampled area show signs of low concentrations of phosphates.

Radiating out from the central enclosure were lines of stakes which coincided, in the mound material above, with clear divisions between different grades and colours of dumped spoil. The stakeholes varied from 2 to $8 \mathrm{~cm}$ in diameter; the great majority were between 4 and $6 \mathrm{~cm}$.

Eight fences were laid out before any major dumping for the construction of the mound took place and were therefore visible over several metres on the OLS. Short lengths of four fences were visible in the OLS near the central enclosure. The suggestion that the short fences represent slightly later structures is discussed on $\mathrm{p}$ 233. A further five fences were not excavated for their full length. Through most of the mound construction the original lines of these fences were observed by the builders as divisions in the mound. It appears that when mound material was dumped to a depth sufficient to bury or almost bury them further fencing was erected on roughly the same line to continue the boundary upwards. In places boundaries disappear at higher levels and new lines appear. In some cases boundaries do not appear on the plans because they could not be seen at a specific level although they were noted at levels above and below (figs 46, 47).

In the SE quadrant where a fine glacially deposited silt, dug from the ditch at that part of the site, was used for much of the later construction, small sections of post and wattle impressions were preserved in the upper part of the mound (Period III, phase C). The fragments of hurdle impressions were not large enough to provide much information about the lengths or heights of the fencing units, presuming that the fences were not built as continuous structures on site. The preserved wattle impressions in the Period III, phase C, part of the mound ( $\mathrm{pl} 14$ ) showed vertically set poles and posts between 2 and $7 \mathrm{~cm}$ in diameter linked by closely spaced wands varying between 1 and $5 \mathrm{~cm}$ in diameter, those between 2 and $3 \mathrm{~cm}$ being most common. The impressions provided no clue to the method of construction, whether the cross-pieces were tied or interwoven, nor were they clear enough to show breaks between any fencing units. In the lines of stakeholes on the old land surface small discontinuities in spacing or alignment may mark the end of individual hurdles. Such discontinuities can be seen on fig 40; they are discussed in more detail on p 237.

To the NE of the enclosure in the NE quandrant near the $\mathrm{N}$ baulk a series of parallel depressions bounded to the $\mathrm{N}$ by a perpendicular depression may have represented the impression of a hurdle laid flat on the ground and surviving until the construction of the mound. The hurdle lay in one of the 'furrows' of Period II. The impression measured $\mathrm{c} 1.4 \mathrm{~m}$ square. To the $\mathrm{E}$ a similar impression, $1.2 \mathrm{~m}$ long and $0.4 \mathrm{~m}$ broad was noted at the $\mathrm{N}$ side of fence number 2 .

The foregoing evidence has been presented with little comment on the sequence in which the various elements were built and used, but some clues exist. It is clear that the groove running E from F1 respected posthole F13. In each case the grooves were used to align a radial fence (nos 6, 11 and 14) but nowhere do the radial fences penetrate the enclosure (with the possible exception of a single stakehole in the SW part). The postholes of the enclosure cut the grooves. The $S$ half of the enclosure was built of more substantial posts than the $\mathrm{N}$, perhaps indicating a chronological difference. The possible building sequence of the sub-mound structures is discussed below ( $\mathrm{p} 232$ ). At the $\mathrm{N}$ edge of the site a charcoal sample was found on the OLS, buried under the turf cap of Period III, phase C. The radiocarbon assay was $1855 \mathrm{bc} \pm$ 100 (GU-1134).

\section{Period III/phase B: the ring-bank and the central enclosure (fig 43a, b)}

In this phase the construction of the mound began. Material was dumped in the bays formed by the radially arranged fences erected in Period III, phase A. Although fence impressions were only rarely preserved the lines of fences within the mound were revealed by vertically defined differences between dissimilar deposits in adjacent bays.

There were at least nine radial fences at the beginning of dumping (fig 40 ; fence nos: $2,4,8,10,13$, 


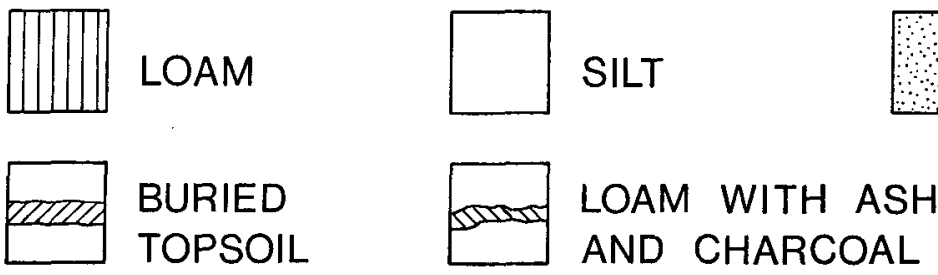

\section{GRAVEL}

\section{TOPSOIL} AND CHARCOAL

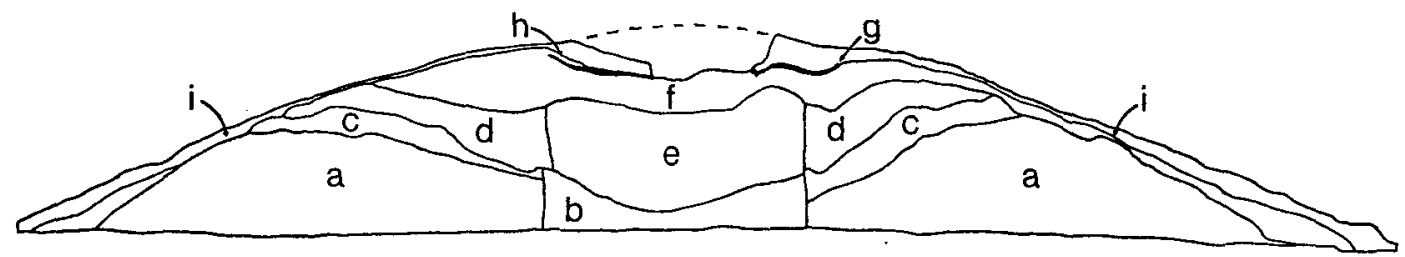

FIG 43 Barrow: interpretive diagram of the main cross-sections. Key: a - ring-bank, Period III/phase B; bprimary fill of central area, Period III/phase B; $\mathrm{c}$ - stone layer, Period III/B/C; d - upper mound material, Period III/phase C; e - stone fill of central area, Period III/C; $f$ - turf, Period III/phase C; $\mathrm{g}$ - activity surface, Period III/phase D; $h$ - fill above ' $\mathrm{g}$ ', Period III/phase E; $\mathrm{i}$-stone capping and topsoil, Period III/phase F.

$15,16,17$ and 22). The stakeholes of these fences were found between the central enclosure and the limit of severe root disturbance near the edge of the mound.

In most of the bays the first deposits were turves dumped at the edge of the mound area (possibly cut from the planned site of the quarry ditch (see below p 237), which provided most of the construction material in this phase). The dumping of gravel dug from the ditch also began at the edge of the mound so that from the first the central enclosure was surrounded by a ring-bank just within the circuit of the ditch. Once this bank had been built up, further material was dumped down its inner face towards the centre. This process continued with the result that the ring-bank grew in height and spread towards the central enclosure. During this process it is possible that four further radial fences were erected (see discussion on $\mathrm{p}$ 233). The line of fence 9 (clearly visible in the mound material) showed on the OLS only as a line of three dimples and one stakehole, suggesting that it had been built once some spoil had been dumped.

Four of the fences (nos 1, 6, 11 and 14) were excavated for limited lengths only and it was therefore impossible to determine whether they were original fences or were erected after the dumping had begun. Three fences (nos 18, 19 and 20) did not appear on the OLS (though 18 may have appeared as two stakeholes to one side of its later line) but were erected during this phase. A further fence in the NE quadrant (no 5) was imperfectly visible on the OLS and possibly reappeared in Period III, phase C. Near the $\mathbf{N}$ baulk a division between two deposits was noted at one level only (no 21).

By the end of this phase about 20 fences had been erected, nine before dumping began, four certainly during early dumping, four in one or other of these sub-phases and three slightly later. Thus the mound was built in a maximum of 20 bays during this phase. The largest bay was that between fences 1 and 2 but the possible presence of fence 21 , the evidence for which was slight, would reduce it to two bays of average size. The smallest were those in the NE quadrant delineated by fences 3,22 and 4 and in the $S$ part by 10,11 and 12 . It should be noted that fences 22,4 and 5 , though visible to a greater or lesser extent on the OLS were not recorded again until the later part of Period III, phase $C$ when they appeared as fences set on top of the filled lower bays of that phase. This, however, is no proof that they did not exist between these two layers, only that they could not be seen.

In the NE quadrant the bay formed by fences 2 and 3 appeared to have been treated differently from early in the construction sequence until late in Period III, phase C. Fence 2 was established before dumping began, fence 3 possibly after a certain amount of dumping had taken place (see above). In this bay the level of dumping may have been kept lower than in the bays to $\mathrm{N}$ and $\mathrm{S}$ (see discussion on $\mathrm{p} 236$ ). 
The ring-bank, constructed mainly using gravel dug from the quarry ditch, was, when completed, a rather irregular structure measuring approximately $20 \mathrm{~m}$ in diameter, peak to peak. It varied greatly in height. The highest portion of the crest was in the NW part of the mound (c $3.5 \mathrm{~m}$ above the OLS). To the SE the level dropped significantly towards the lowest point $(\mathrm{c} 1.8 \mathrm{~m})$ in the bay between fences 7 and 8 . Figure 48 illustrates the approximate shape of the ring-bank after the deposition of the stone layer which marks the boundary between the IIIB and IIIC phases of construction (infra p 234). The material of the ring-bank rose to a height of between 0.5 and $1.4 \mathrm{~m}$ against the fence of the central enclosure. The enclosure was partly filled with material dumped against the inner face of the timber wall. The relationship of this fill to the ring-bank is discussed below ( $p$ 233). The embanking material within the enclosure was of two kinds; in the NW half it was entirely of gravel; to the SE it included large quantities of the natural fine silt. The two materials met along a 'valley' in the deposit running SW-NE; at the NE end the level of the embanking against the timbers was at its lowest $(\mathrm{c} 0.8 \mathrm{~m})$ and next to the bay formed by fences 2 and 3 (and the arrangement of stakes and grooves at the NE of the enclosure on the OLS) the embanking ceased altogether, leaving a hollow in the material against the timbers. The dump was highest at the NW (c $2.4 \mathrm{~m}$ ), where the ring-bank was also highest, but it was higher than the stone layer which lay on the ring-bank surface, a layer which roughly coincided with the top of the embanking elsewhere. However, the embanking was of two types deposited in different ways and it is suggested that the lower part can be assigned to IIIB and the upper to IIIC (discussion on $p$ 231).

At the $\mathbf{S}$ side of the embanking material the original silt and gravel dump was covered by a thin layer of water-washed silt, apparently from the body of the ring-bank to the S. The N-S section (A-B) on fig 44 shows the average height, the E-W section (C-D) almost the extremes, although, as has been mentioned above, the upper 0.8-1.4 m of the dump at the $\mathrm{N}$ and $\mathrm{W}$ may be secondary. Any deposits left on the OLS within the enclosure were at this stage covered with soil for the first time. The volume of material in the ring-bank has been calculated as between 1200 and $1450 \mathrm{~m}^{3}$, that of the dump in the central enclosure approximately $35 \mathrm{~m}^{3}$. Apparently all of this was quarried from the ditch, including the small quantities of turf found at the base of the ring-bank. A sample of charcoal sealed in the IIIB mound material under the stone layer in the NW quadrant was submitted for radiocarbon dating. The assay was $1785 \mathrm{bc} \pm 85$ (GU-1103).

Finds Within the body of the ring-bank a number of chipped stone artefacts and waste pieces as well as sherds of pottery were found. The origin of the pieces, coming from such a mixed deposit, was not clear. Likewise the sherds of food vessel pottery (SF25) found, along with a quartz inner flake, in the internal embanking of the central enclosure, may have originated in the soil around the mound, rather than in the period of mound construction.

\section{Period III/phase B-C boundary: the stone layer (fig 43c)}

Though at the end of the ring-bank construction the levels of fill in the various bays were slightly different, a distinct and possibly uniform event was marked by the deposition, in every bay, of a layer of small boulders (see sections on fig 44). Some of these deposits were arranged relatively uniformly over the bays, others were dumped rather more to one side of the bay than to the other. The figurative plan (fig 48) cannot show sufficient detail but it was clear during the excavation that the fences were still visible and were largely respected by the stone dumps. Plate 15 a shows the stone layer at the peak of the ring-bank in the NW quadrant; the layers run downwards (not exposed in photograph) towards the centre of the mound (to the right).

This episode has been taken to mark the boundary between phases $\mathrm{B}$ and $\mathrm{C}$ of the construction. There is no proof of a break in the work, but different dumping techniques and, increasingly, different materials were used in the upper part of the mound, above the stone layer (see below p 235). The stone lenses in some cases mounted higher up the fence of the central enclosure than the internal embanking, and in others the top of the stone deposit coincided with the top of the embanking.

The layer was made of approximately $175 \mathrm{~m}^{3}$ of stone, which showed signs of sub-aerial erosion and may therefore have originated as field-stone.

\section{Period III/phase C: the mound above the stone layer (fig 43d, e, f)}

Subsequent to the deposition of the stone layer in all the bays, the dumping of material dug from the quarry ditch continued. While in Period III, phase B most had been gravel, a greater proportion of natural 
fine green silt was now used in the $S$ part of the mound, reflecting changes in the fluvio-glacial deposits excavated as the ditch deepened (see soil report p 260). In one bay, between fences 9 and 10 in the SE quadrant, stone was used exclusively for a short time at a higher level (pl 14d). The arrangement of bays formed by radial fences continued. Given the height of the ring-bank at the end of IIIB it seems certain that the original fences had had to be replaced as the mound grew. This process may be seen in the slight changes in the fence lines (between different fills) at different levels, though such relatively small movements could have resulted from the distortion of fences under load. Fence 11 was not seen above the stone layer; however, the difficulties of finding the boundaries between deposits were such that the absence of proof was not proof of the absence of a fence. In the silt in the SE quadrant, fragments of the vertical impressions of three of the fences were found (fence nos 7,8,9). An insufficient area was preserved to allow any conclusions about hurdle sizes to be drawn and the impressions themselves had been distorted and disturbed by later root activity. However, a general impression of the style of fencing can be had from the illustration (pl 14b).

Very different types of fill were deposited inside and outside the central area, which was defined by a clear vertical break between them. Outside the centre, silt and gravel were dumped (the former concentrated in the $S$, the latter found all round the mound). At the $S$ during this dumping some silt was washed over the embanking of the central enclosure. In the $\mathrm{N}$ and $\mathrm{W}$ parts of the enclosure, gravel was probably deposited over the primary dump of material against the timber wall. A clear difference between the upper and lower deposits was noted (see fig 44; C-D section). Subsequently the fill of the central area was exclusively stone, generally similar in size and appearance to the stone layer of the IIIB/C boundary. Within this material, near the top of the silt and gravel embanking of the central enclosure, a modern iron shovel was found; the relic of an, as yet undated, disturbance which took the form of a shaft dug from the top of the mound through the stone core. The upper part of the shaft was defined in section by the admixture of humus to the stone core. The precise extent of the disturbance could not be defined, but the vertical border between the materials inside and outside the central enclosure was undisturbed and the surviving extent of a layer (Period III, phase D) deposited near the top of the mound, suggested that the hole had been only 4-6 $\mathrm{m}$ in diameter and had narrowed rapidly as it deepened.

The material outside the central area was dumped both downhill from the crest of the ring-bank towards the centre (figs $43 \& 44$ ) and in horizontal layers. In the S, where silt predominated as the building material in the lower part of the Period III, phase C mound, ditch-quarried spoil was superseded in the latter part of the phase by turf, especially in the SE quadrant. It was clear that the fence system was still used; in the SW quadrant fence 19 separated two bays, one filled with turf, the other with silt. Eventually turf superseded quarried material over the whole mound, at first laid within the bays, later, as the working area on top of the mound shrunk, without a fencing system. It can be seen clearly on the mound crosssections that the neatly defined vertically sided core of stone ceased a maximum of $2 \mathrm{~m}$ below the top of the mound, though that part of the mound has been disturbed. The mound was completed to the end of IIIC using a mixture of turf and stone, apparently not dumped in bays. A slight irregular hollow was left in the top of the mound at the end of this phase; the next phase of activity (IIID) took place in this hollow.

A layer of turf was also placed over a large part of the mound's surface. The cross-sections show that in places the shoulder of the mound, at the time of excavation, was almost bare of turf and that the layer was broken into two parts, one on top of the barrow and the other round its skirts. It could not be determined if this was the original arrangement or whether a continuous turf layer had eroded at the vulnerable shoulder.

The material dumped in Period III, phase $\mathrm{C}$ can be split by origin into three parts. Firstly, material quarried from the ditch (approximately $250 \mathrm{~m}^{3}$ ); secondly turf, presumably cut from around the site (approximately $360 \mathrm{~m}^{3}$ ) and lastly the stone used to fill the central enclosure (approximately $100 \mathrm{~m}^{3}$ ).

Throughout Period III, phase C, at least until the abandonment of the system of bays, the bay between fences 2 and 3 seemed to be filled differently from the others. The level of the stone layer, which was placed in all the bays at the boundary of phases $B$ and $C$, was lower in this bay than elsewhere. Likewise when the use of turf superseded quarried material in the upper mound, this bay had been filled to a lower level than the others with quarried spoil. Figure 49 is a diagrammatic section, a chord of the barrow, seen from the NE, illustrating the relative levels of fill in this bay and its neighbours.

Finds Within the topmost material of this phase a spindle-whorl of siltstone was found (SF114), apparently in an undisturbed context under the Period III, phase D, layer. This, and the other material 


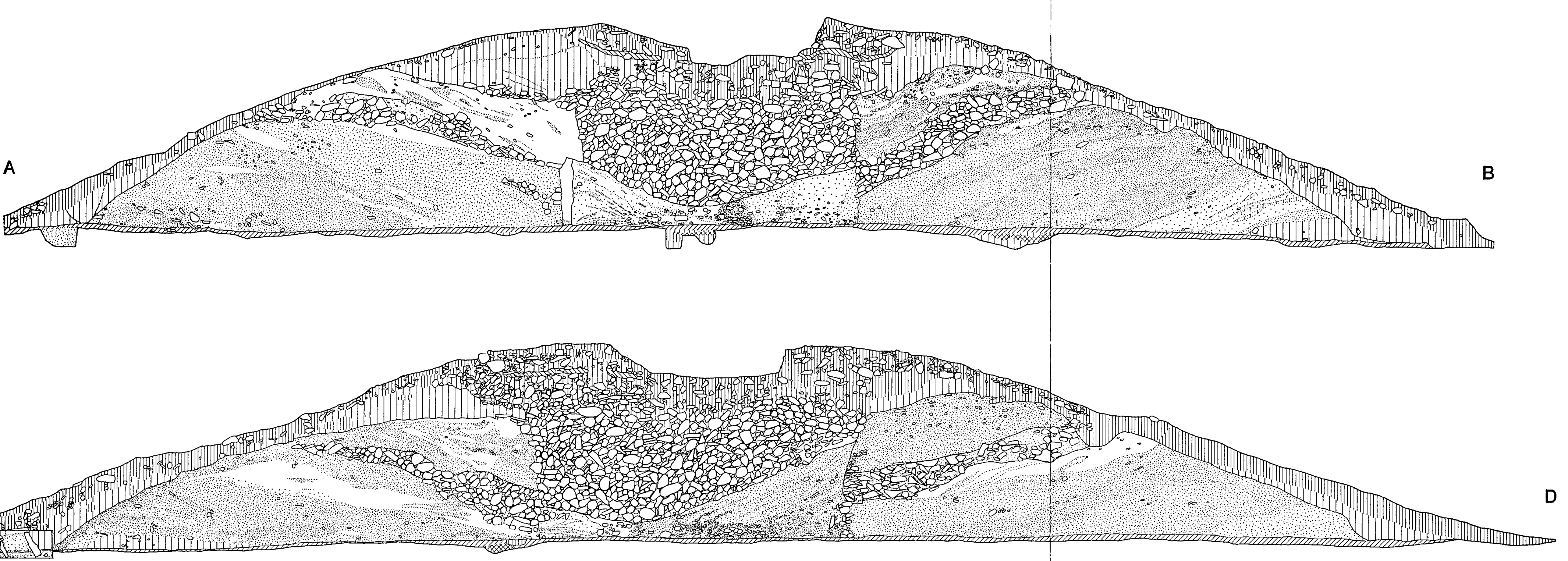

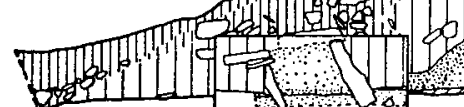$$
\text { 。 }
$$
Fro 44 Barrow main cross-sections (see fig 40 for their location)

NORTH MAINS BARROW : Main Sections 
E

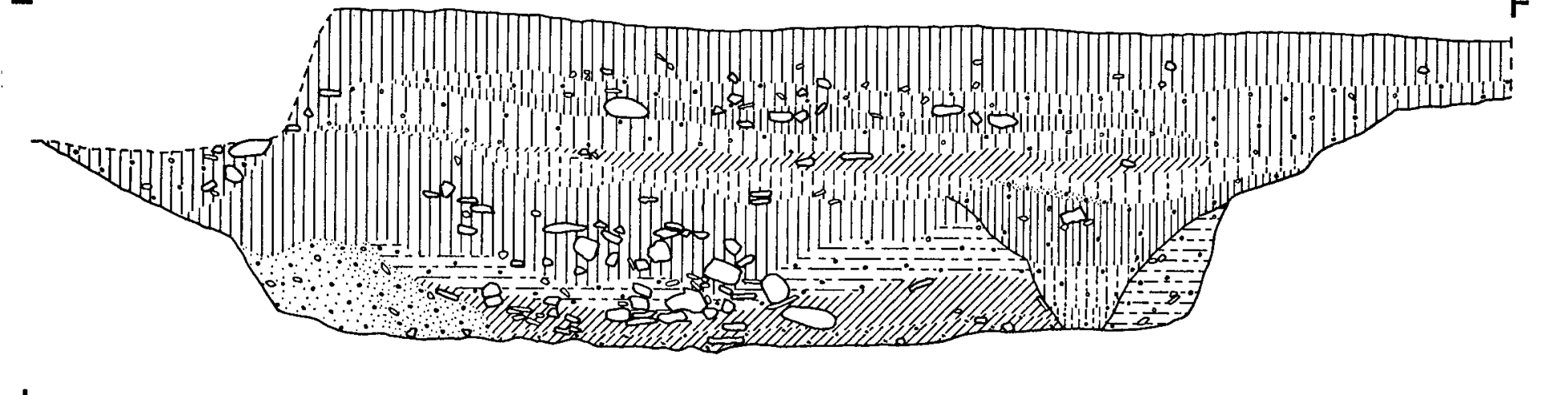

$J$

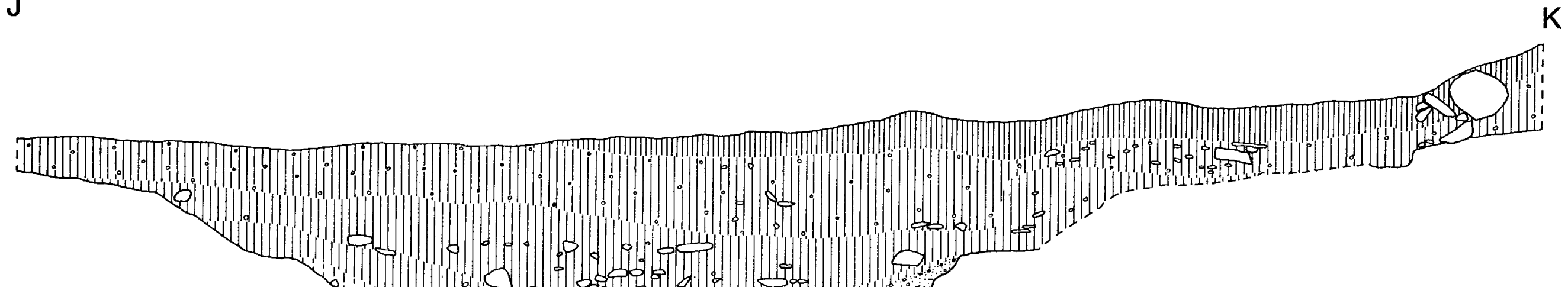

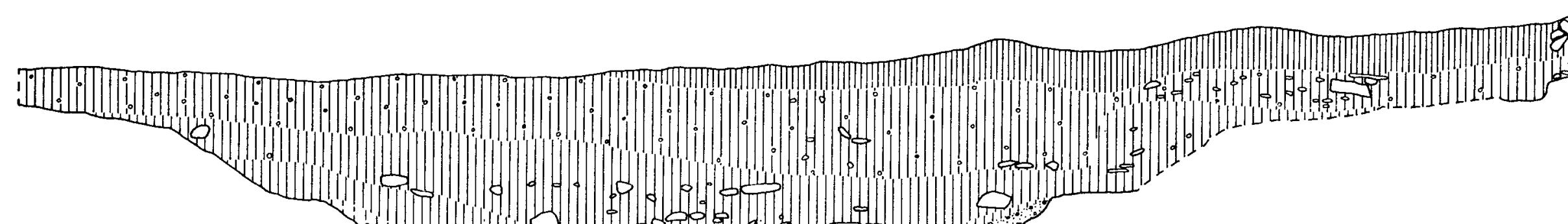

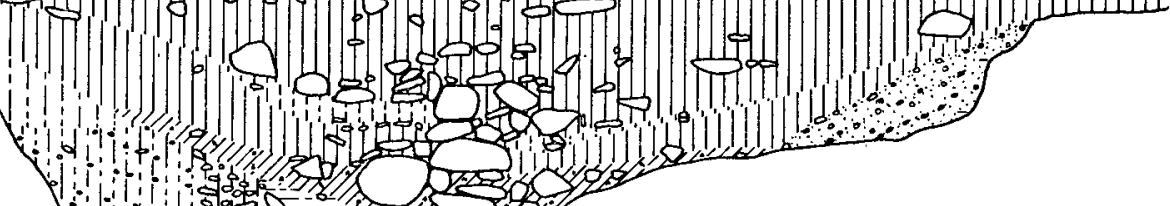

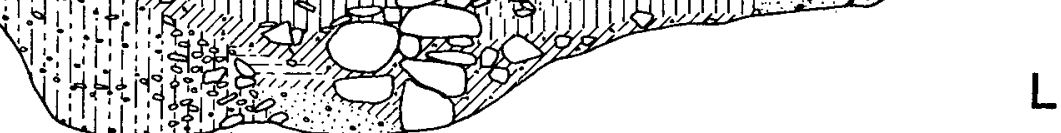

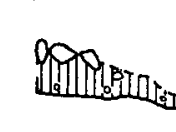

G

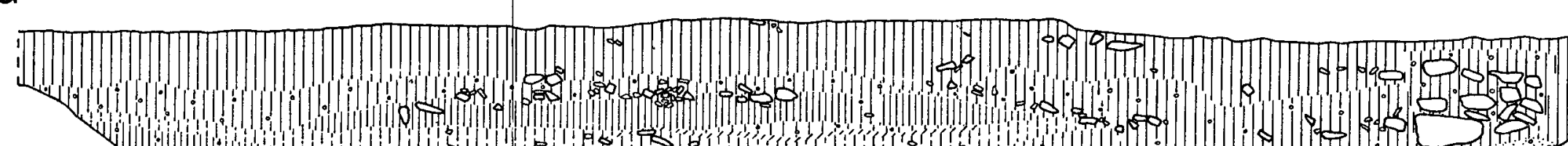

(1) -

So
K
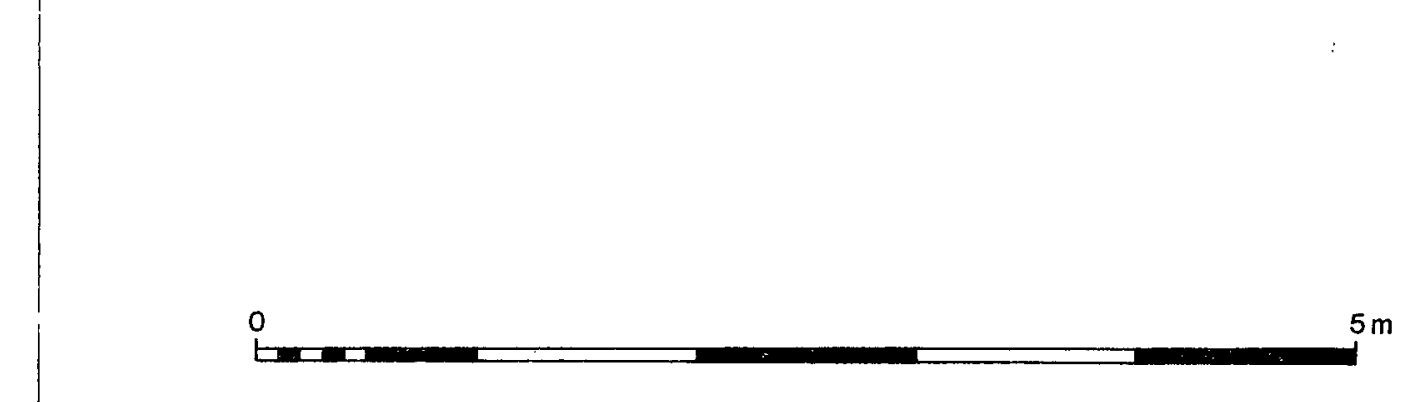
from the Period IIIC mound structure, was once again of uncertain origin because of the possibilities of inclusion of artefacts of substantially earlier periods. The artefacts are described in their separate catalogues.

\section{Period III/phase D: use of the hollow on top of the mound (fig 43g)}

At the end of Period III, phase C a hollow some $95 \mathrm{~m}^{2}$ in extent was left in the top of the material of the mound. Within the hollow there was a fine grey, heavily charcoal-stained layer, apparently an ashy deposit which only survived in patches because of recent disturbance. Mixed charcoal (ulmus and quercus) from immediately below the layer was submitted for radiocarbon dating. The assay was $1665 \mathrm{bc} \pm 85$ (GU-1102). No charcoal suitable for dating was found in the layer itself and no finds were recovered.

The activity which occurred on the surface, apart from the apparent setting of one or more fires, was not elucidated by excavation. It may be that the destruction of the central part of the hollow by the modern shaft removed the hub of the activity. The cover slab of burial $\mathrm{C}$ lay immediately below the projected surface of the hollow, though the precise context of the burial was destroyed by the growth of a tree immediately above it. It was therefore not possible to determine whether the cist was inserted from the surface of the hollow or from the top of the mound.

\section{Period III/phase E: completion of the mound (i) (fig 43h)}

Subsequent to the IIID activity the hollow was filled and the top of the mound was rounded off with a mixed deposit of turf and stones (approximately $195 \mathrm{~m}^{3}$ ). Much of this material was disturbed by the recent shaft cut into the mound. Only one artefact, a sherd of beaker pottery (SF40) was recovered.

\section{Period III/phase F: completion of the mound (ii); the stone capping (fig 43i)}

The last recognizable phase of activity on the mound saw the covering of the whole surface with a single layer of cobbles. As a result of the root and animal disturbance, especially in the NE quadrant, this cladding was preserved patchily. Plate 15a illustrates the NW quadrant where the capping was best preserved.

In the SE quadrant a single line of more substantial boulders was noted approximately $2 \cdot 7 \mathrm{~m}$ up from the bottom of the mound, within the capping, running around the mound from the $\mathrm{E}$ baulk for a distance of $6.5 \mathrm{~m}$.

On the top of the mound larger boulders were used for the capping. They were set densely in a circular area c 14-15 $\mathrm{m}$ in diameter which was preserved well only in the SW quadrant. Elsewhere natural and human disturbance had severely disturbed this feature. Burial $\mathrm{A}$ was constructed within the stone layer in the SE quadrant, downslope from this larger stone setting.

The nature of the layer and the later disturbance prevented any attempt to relate finds to this phase of activity or to determine its relationship to the burials dug into the surface of the mound. Approximately $330 \mathrm{~m}^{3}$ of stone was used to cover the $c 1320 \mathrm{~m}^{2}$ surface of the mound.

\section{THE CUPMARKED STONE (pl 15c)}

In the shallow pit dug in 1957 a large cupmarked slab was found lying decorated face down, apparently left in that position after the earlier undated disturbance. After the 1957 excavation the slab was left lying decorated face upwards at the edge of the pit. There were 30 complete or unfinished cupmarks on one surface. In addition there were a number of groups of peck-marks, perhaps the beginnings of cupmarks. The slab measured approximately $2.1 \mathrm{~m}$ long by $1.6 \mathrm{~m}$ broad (both maximum figures) and $0.2 \mathrm{~m}$ thick. The stone was severely damaged some months after the completion of the excavation and has since been removed by SDD (Ancient Monuments) to a place of safety. Any evidence of a relationship between the cupmarked slab, burial $\mathrm{K}$ and the stone capping of the mound was destroyed by the earlier excavations (see p 189).

\section{THE DITCH (figs 40, 45)}

Before excavation began no trace of a quarry ditch was visible except to the NW of the mound, where a hollow $17 \mathrm{~m}$ long $(\mathrm{N}-\mathrm{S}), 12 \mathrm{~m}$ broad $(\mathrm{E}-\mathrm{W})$ and up to $1.5 \mathrm{~m}$ deep was clearly visible. On the outer, W, edge of the hollow had been a line of trees, which survived only as stumps in 1978. It is likely that the hollow, which on excavation was proved to be part of the mound ditch, survived because the trees 

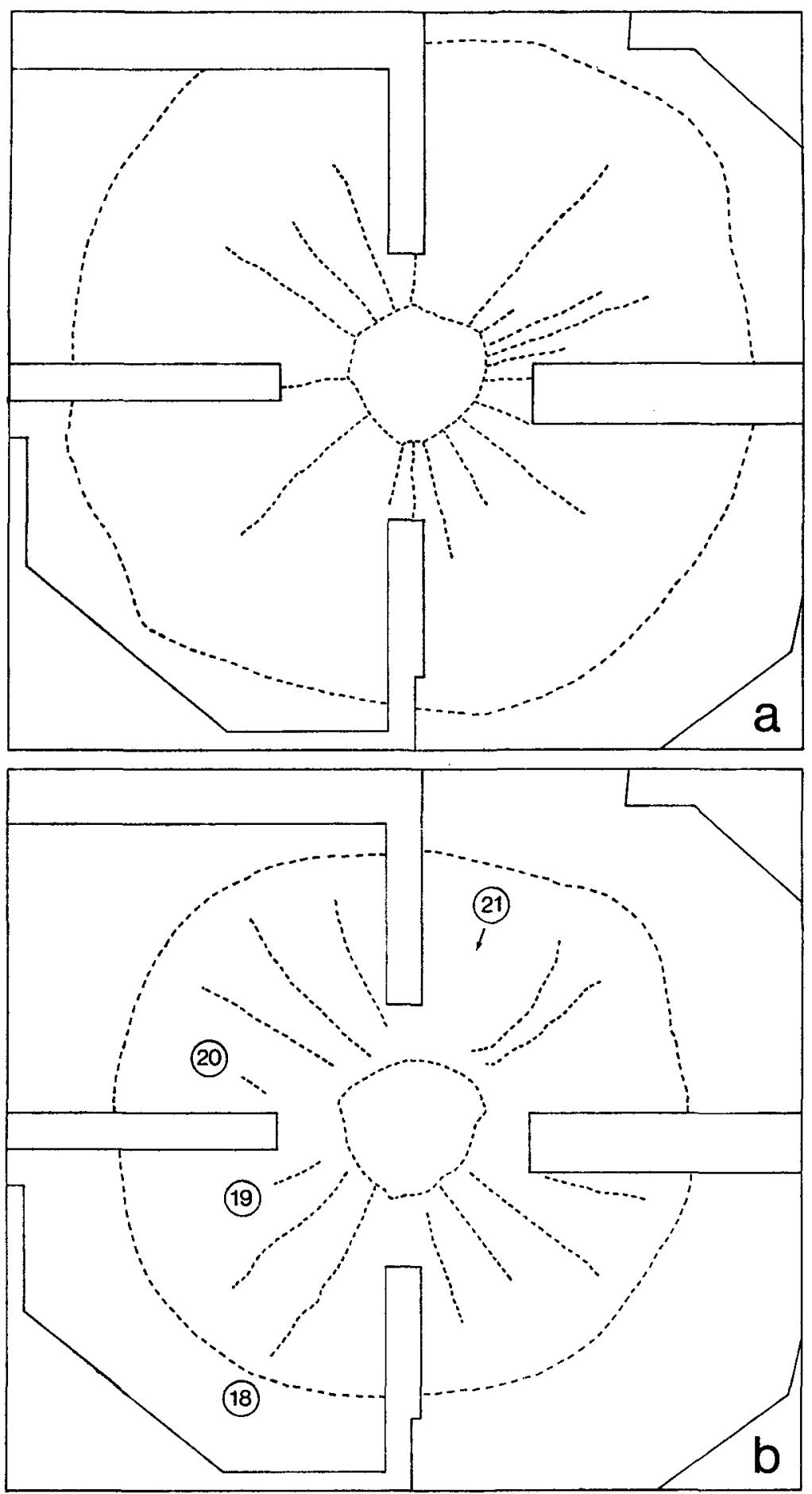

FIg 46 Barrow: diagrams of fence lines within the mound: $a$ - at the level of the old land surface; $b-1 \mathrm{~m}$ above the OLS (ringed numbers label fences not already labelled on fig 40) 

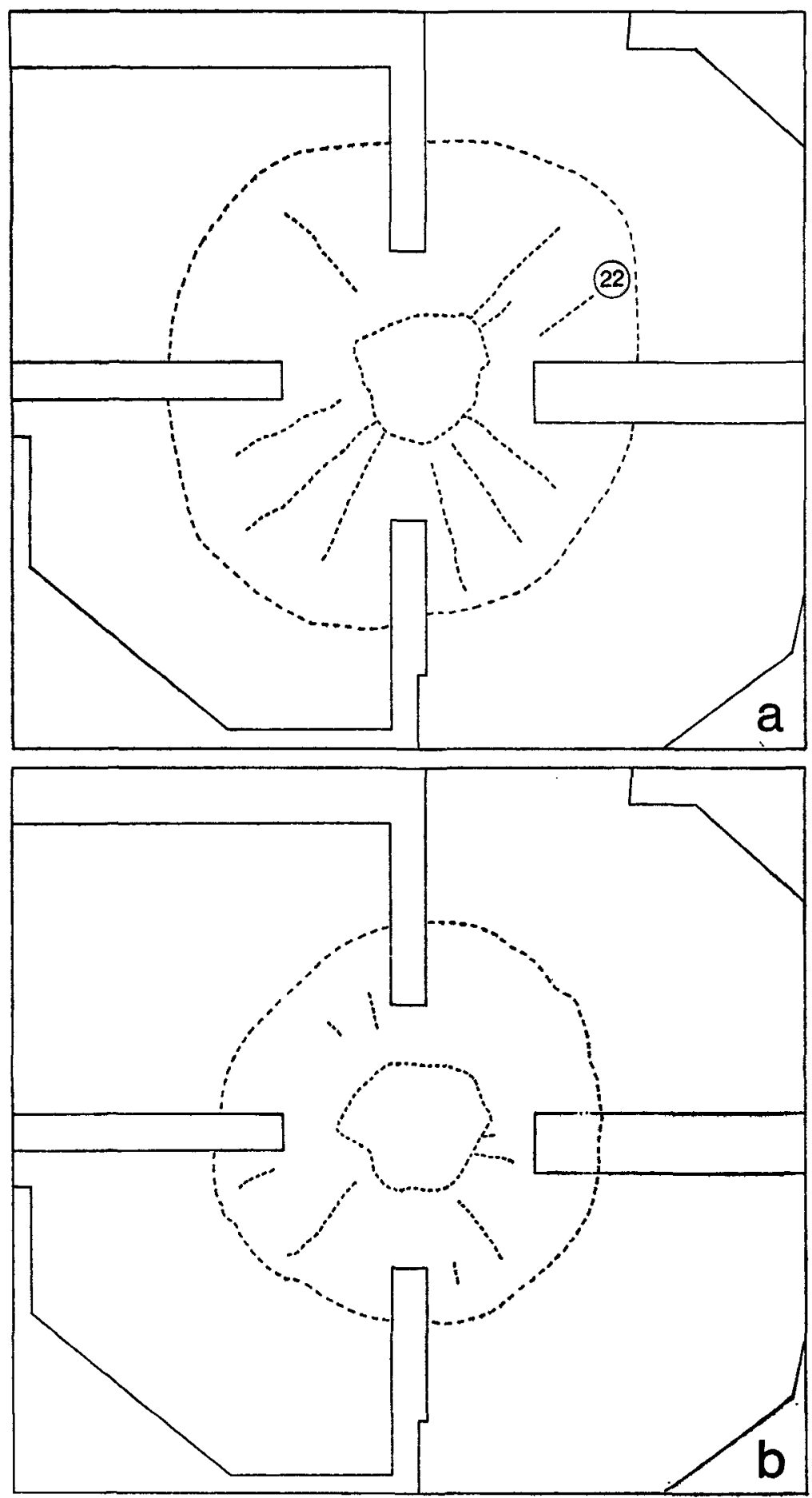

FIG 47 Barrow: diagram of fence lines within the mound: $\mathrm{a}-2 \mathrm{~m}$ above the OLS; $\mathrm{b}-3 \mathrm{~m}$ above the OLS (the ringed number labels a fence not already labelled on fig 40) 
prevented the encroachment of agricultural activity, which had effectively obliterated surface indications of the ditch elsewhere. The area of the hollow between the $W$ baulk and the modern wall was excavated as fully as the tree stumps allowed. Further sections were dug at the NNE and at the S; $22.5 \mathrm{~m}$ of ditch was excavated, about $15 \%$ of the total $(\mathrm{c} 150 \mathrm{~m})$.

In the NW cutting the ditch was between 8 and $10 \mathrm{~m}$ broad and for the most part sloped gently from the $\mathrm{W}$ to a depth of approximately $1.8 \mathrm{~m}$ below the old land surface under the barrow, only the lowest part of the profile being steeply sided. To the E, towards the mound, the side was steeper. Its shape was quite uniform throughout the $12 \mathrm{~m}$ length of the cutting and no segmentation was visible, even to the limited extent found in the S cutting. The ditch was cut entirely through gravel.

In the NNE cutting a substantial ridge rising some $0.6-0.7 \mathrm{~m}$ above the ditch floor and measuring c $0.8 \mathrm{~m}$ across bisected the excavated length of ditch $(6.5 \mathrm{~m})$ from $\mathrm{N}$ to $\mathrm{S}(\mathrm{pl} 15)$. The ditch was $\mathrm{c} 1.7 \mathrm{~m}$ deep below the old land surface and between 8 and $9 \mathrm{~m}$ broad and its sides were much steeper than in the NW cutting. A relatively modern ditch, probably the same feature which ran across the henge, had severely disturbed the $\mathrm{N}$ edge.

In the cutting at the $\mathrm{S}$ edge of the mound the ditch was steep on its outer edge and shallow on its inner. It was up to $2 \mathrm{~m}$ deep below the OLS, and approximately $8.5 \mathrm{~m}$ broad. The bottom of this $4 \mathrm{~m}$ wide section was divided by a low ridge, possibly a very much smaller equivalent of the division between ditch segments at the NNE. The lowest $0.3 \mathrm{~m}$ of the ditch had been dug through the fine glacial silt which was a major feature of the mound in the SE quadrant.

In all three cuttings the ditch appeared to have filled naturally. The gap between mound and ditch was small and in the $\mathbf{S}$ cutting especially some mound material had slipped into the ditch. The finds from the ditch fills were of insecure context, because of the mixed nature of the deposits in which they were found; they are listed separately in the finds catalogues. Briefly, the pottery assemblage consisted of sherds from possibly three vessels. From the first there were two undecorated sherds (SF43; SF44), from the second an abraded sherd and two fragments (SF46), and from the third an abraded, lugged sherd (SF45). A small cupmarked stone (SF113) was also recovered. Sherds SF43 and SF44 were found some distance apart and at different levels within the fills of the ditch (in the NW cutting), the latter being in a possible primary context, the former in a secondary fill. The remainder of the finds were recovered from secondary fills.

From the excavated portion of ditch it has been calculated that approximately $1540 \mathrm{~m}^{3}$ of gravel and silt were quarried from its entire length. The volume of the mound has been calculated as $3345 \mathrm{~m}^{3}$, the extra material being turf, stone and mixed soils. The $\mathbf{N}$ part of the mound was built predominantly of gravel, with the addition of the ubiquitous stone layer at the end of Period IIIB, though in the upper mound in the NE quadrant one bay was filled in part with the fine green silt. In the SE quadrant much silt was used after the deposition of the stone layer, giving way to turf above. In the SW quadrant some silt was used in the construction, also giving way to turf. It seems likely therefore that in the S part of the mound, and at one point in the NE, the ditch was dug deep enough to strike layers of the silt. On digging a test pit in the SE quadrant from the OLS Romans and Robertson found a natural silt layer at a depth of $1.7 \mathrm{~m}$ below the surface, and on the $\mathrm{E}$ side just outside the ditch the silt was found by auguring at less than a metre below the modern topsoil surface (p 260); it is likely, therefore, that some unexcavated sections of the ditch cut such shallow silt deposits (see p 235). Where most silt was used, in the SE quadrant, turf replaced it as a building material at a lower level than in the $W$, where gravel was used. It is suggested that this may have reflected the difficulties of quarrying the silt in wet weather. When fully excavated the silt-bottomed ditch cutting flooded while the gravel-bottomed ones did not.

\section{BURIALS NEAR THE MOUND SURFACE}

Evidence for 10 burial deposits was found within the material of the mound. Two were inhumations ( $E$ and $G$ ) the remainder cremations, showing considerable variety in their method of deposition (A, B, $\mathrm{C}, \mathrm{D}, \mathrm{F}, \mathrm{H}, \mathrm{J}$, and $\mathrm{K})$. Because of widespread disturbance by animal burrowing and tree roots the context of none of these burials was entirely clear. However, termini post quos could be established for the majority.

Burials $\mathrm{E}, \mathrm{G}, \mathrm{H}$ and $\mathrm{J}$ postdated the covering of large parts of the mound with turf at the end of Period III, phase C and therefore date from the end of or after that phase. Burials B and D may have been inserted during the turf deposition or, perhaps more likely, later. Burial A was contemporary with or later than the stone capping of the mound as it lay within that layer, and burial $F$ postdated it, as part of the stone layer had been used for packing. Burial $\mathrm{K}$ was represented only by a scatter of burnt bone in the disturbed part of the stone core of the mound. It could have dated from IIIC onward. 
$\mathrm{B}$

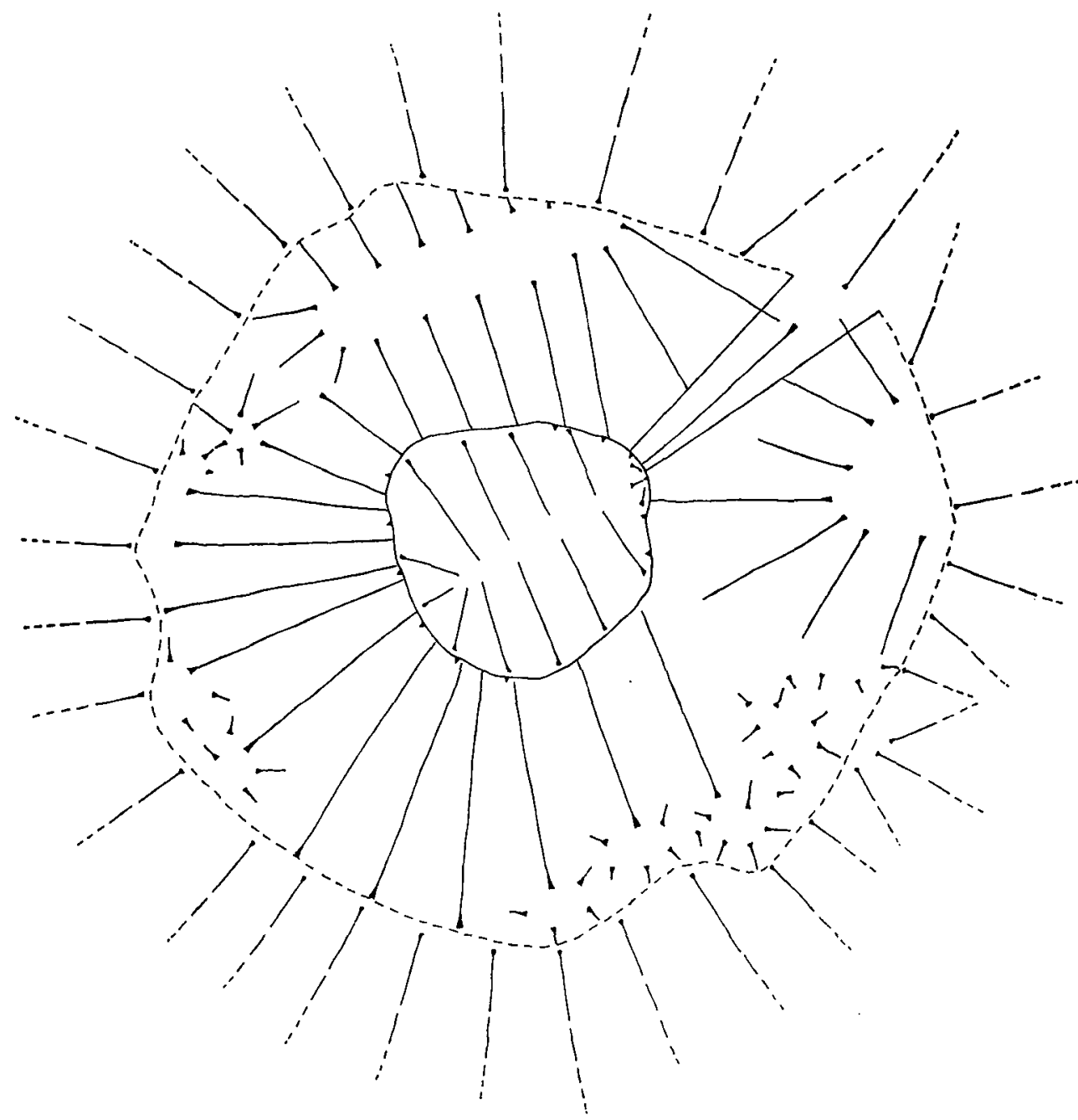

A

FIG 48 Barrow: diagrammatic plan of the barrow after the deposition of the stone layer which marks the boundary between phases B and C of Period III (A-B marks the location of the section illustrated in fig 49)

A

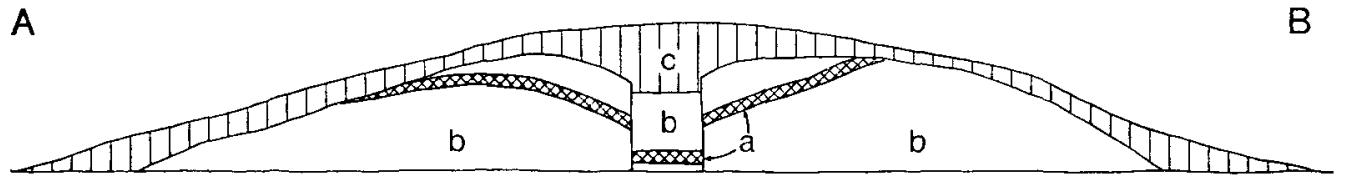

FIg 49 Barrow: diagrammatic section, constructed from plan information, through the 'access' bay between fences 2 and 3; the levels of stone (a), gravel (b) and turf (c) fills are lower in this bay than in the others. The line of the section is shown in fig 48 


\section{TABLe 6}

Barrow secondary burials

\begin{tabular}{|c|c|c|c|c|}
\hline Burial & Inhum/crem & Number & Sex & Age \\
\hline A & Cremation & $4-5$ & $\left.\begin{array}{r}\text { 2-3 Male } \\
1 \text { Female }\end{array}\right\}$ & $\begin{array}{l}\text { 3-4 Adults } \\
1 \text { Child }\end{array}$ \\
\hline B & Cremation & 4 & $\left.\begin{array}{l}1 \text { Male } \\
1 \text { Female }\end{array}\right\}$ & $\begin{array}{l}2 \text { Adults } \\
1 \text { Immature } \\
1 \text { Infant/neonate }\end{array}$ \\
\hline C & Cremation & 8 & $?$ & $\begin{array}{l}4 \text { Adults } \\
1 \text { Adolescent } \\
1 \text { Late childhood } \\
\text { I Early childhood } \\
1 \text { Foetal/birth }\end{array}$ \\
\hline D & Cremation & 7 & $\left.\begin{array}{l}1 \text { Male } \\
1 \text { Female }\end{array}\right\}$ & $\begin{array}{l}\text { Poss } 2 \text { or more adults } \\
\text { Remainder: foetal to adult }\end{array}$ \\
\hline $\mathrm{E}$ & Inhumation & 1 & Child & Child (4-6 or younger) \\
\hline $\mathbf{F}$ & Cremation & 1 & Female & Young adult \\
\hline G & Inhumation & $1 ?$ & $?$ & $?$ \\
\hline H & Cremation & 2 & $\left.\begin{array}{l}1 \text { Female } \\
1 \text { Male }\end{array}\right\}$ & Adult \\
\hline $\mathbf{J}$ & Cremation & 1 & ? Female & Adult \\
\hline $\mathbf{K}$ & Cremation & 1 & ? Female & Adult \\
\hline
\end{tabular}

Interment type
Small rectangular cist

Associations

Polygonal cist

Flint slug knife (SF108)

Flint knife (SF107)

Massive rectangular cist Flint flake (SF109)

Rectangular cist

Massive rectangular cist

Food vessel (SF41)

Flint scraper (SF110)

Rectangular cist

Pit

Food vessel (SF42)

Jet necklace (SF113)

Polygonal cist

Cupmark on base slab
Pit 
One of the inhumations (E) was contained in a substantial cist; the other (G) lay $4 \mathrm{~m}$ to the $\mathrm{N}$ in a shallow uncisted grave. They lay just to the $\mathrm{N}$ of the $\mathrm{E}$ baulk in the NE quadrant close to the edge of the mound.

The cremation burials were of two kinds; the burial of an individual and the burial of a number of bodies. Members of the first group were buried in a rectangular cist $(F)$ and in a small pit, on a flat stone stone $(\mathrm{J})$. The third example, burial $\mathrm{K}$, had been disturbed and scattered.

The members of the second group were contained in a variety of cist-types, ranging from the large rectangular (C and D) and very small rectangular $(\mathrm{A})$ to polygonal (B and $\mathrm{H})$. The extent to which the burials were multiple deposits is noted briefly below in the description of the features (see table 6); more detail is given in the cremated bone report by Denston (p 227).

No pottery was found with the cremations but both inhumations were accompanied by a food vessel (E: SF41; G: SF42); burial E also had a distal-end scraper (SF110), and burial G a disc-bead jet necklace (SF113). Two of the cremations had burnt flint pieces within the bone deposit. Two retouched pieces (SF107; SF108) were found with burial B and a broken retouched piece (SF109) with burial C. fig 50 .

A general guide to the location of the burials and their level above the old land surface is given in

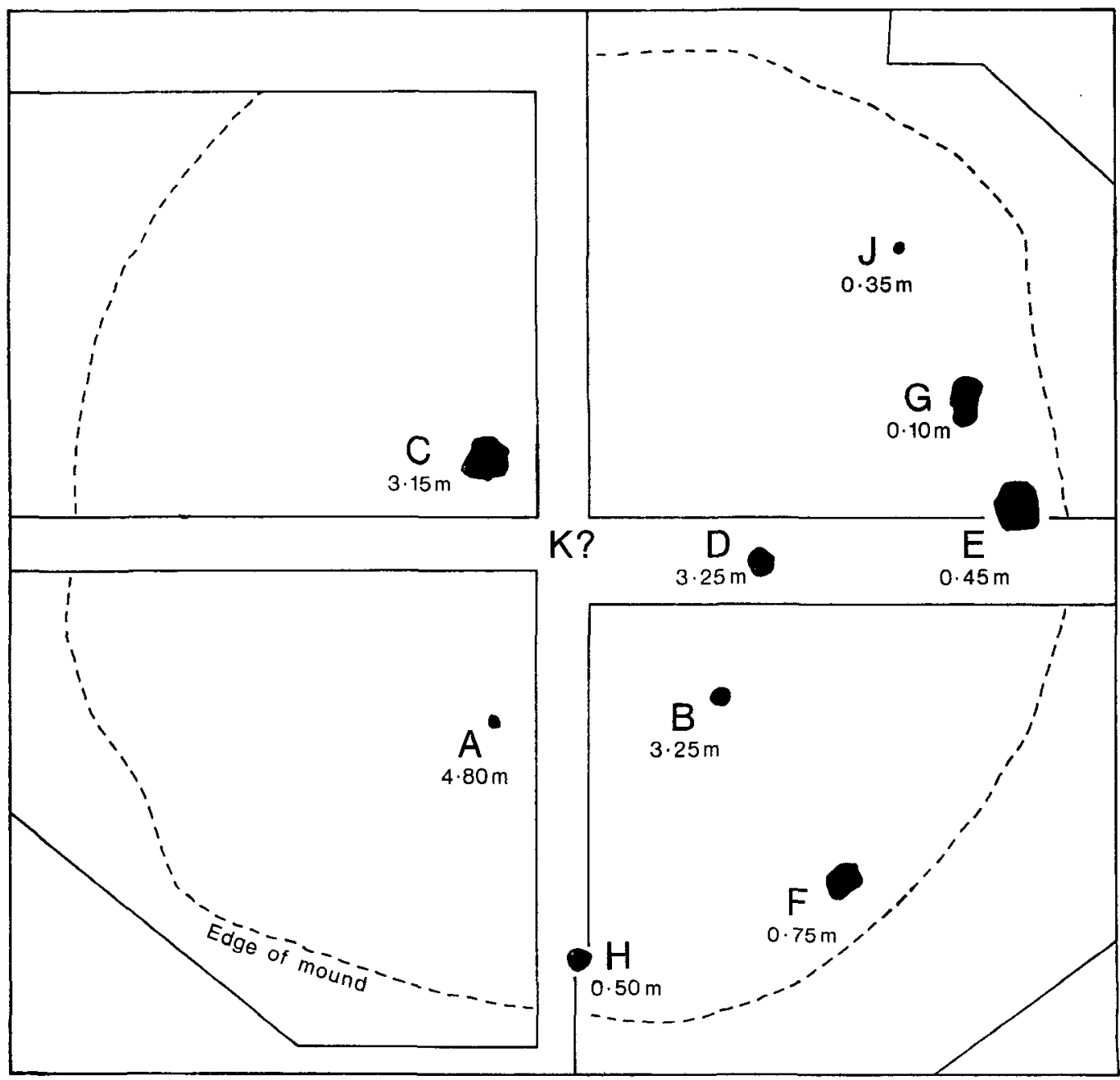

FIG 50 Barrow: plan showing position and height in mound of secondary burials (Heights are relative to OLS) 
Burial $A$ (fig 51) (Cremation p 227)

This cist was set in the stone covering of the mound (Period III, phase F) in the SW quadrant and was therefore contemporary with or later than this phase of activity. It measured approximately $0.25 \mathrm{~m}$ long and $0.2 \mathrm{~m}$ broad and was made up of four irregular slabs set on edge, without base or cover slabs. The cremated deposit, apparently incorporating several individuals, was unaccompanied. The bodies burned had been of a child of 4-5 years plus three adult males and an adult female, or two adult males and an adult female.

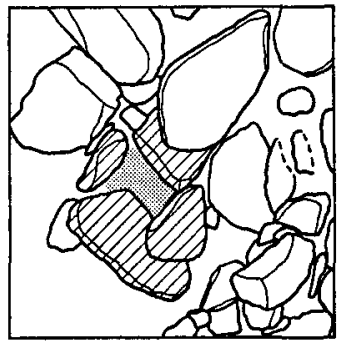

Burial A
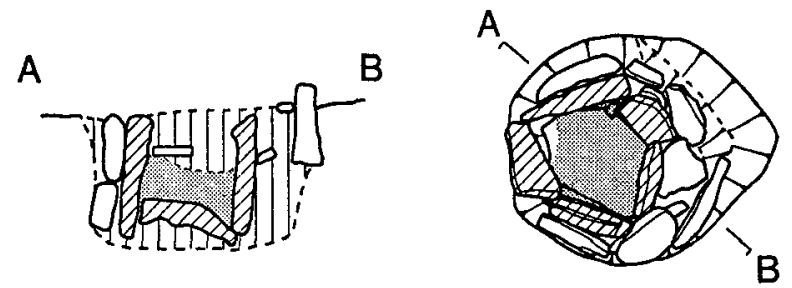

Burial B
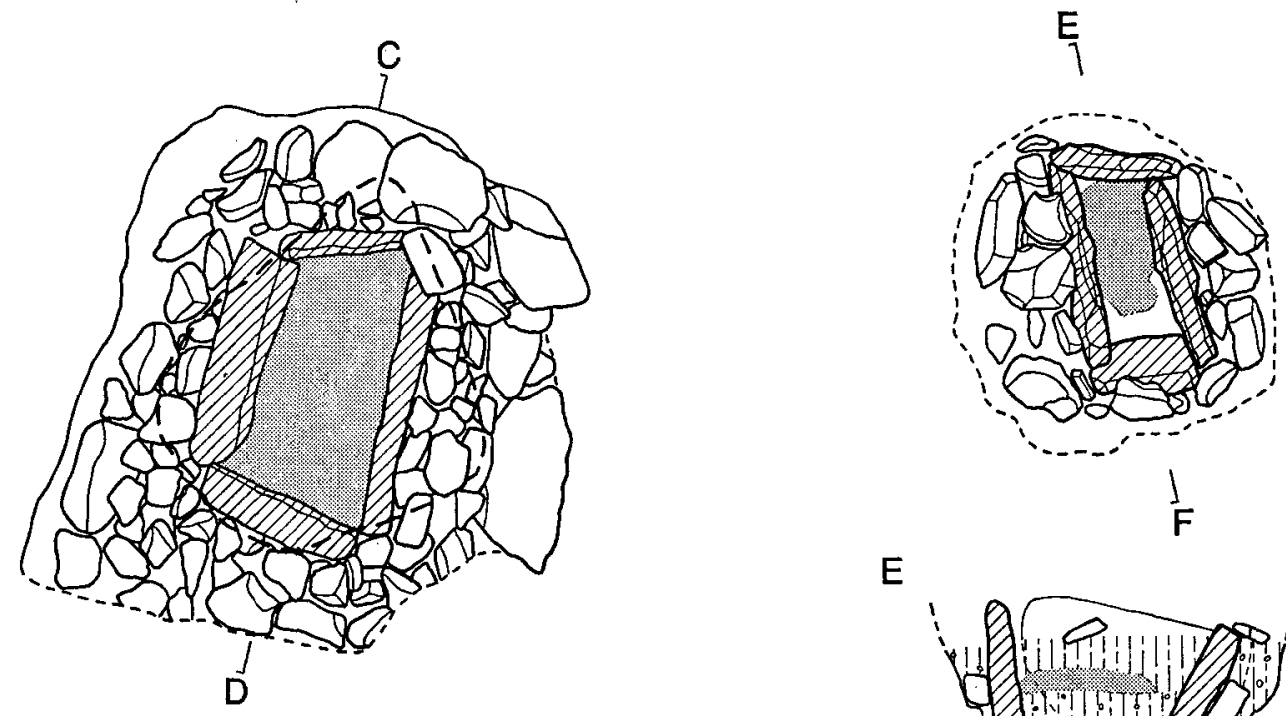

E

F

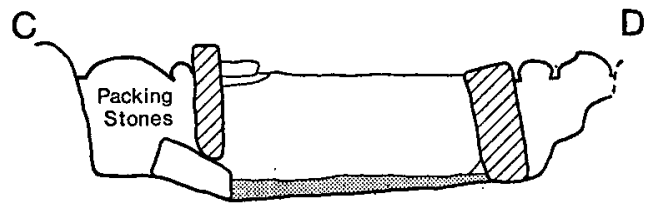

Burial D

Burial C

0 $1 \mathrm{~m}$

Fig 51 Barrow: Burials A-D 
Burial B (fig 51) (Cremation p 227; Stone p 219)

The polygonal cist containing the cremated deposit had been cut into the turf used to complete the mound in the SE quadrant. It was between 0.3 and $0.35 \mathrm{~m}$ in diameter and a minimum of $0.25 \mathrm{~m}$ deep. It was five-sided and each slab was packed in position by slabs set vertically behind it. There was a base slab but no single cover slab. Instead, a ring of flags was laid over the edge slab, partly covered by a second ring above it. The base slab had been inserted after one or possibly two of the side slabs had been positioned. The cremation deposit was arranged in a spiral through the lower fill of the cist, suggesting perhaps that it had been contained in a long bag deposited while the filling of the cist was taking place. The deposit included parts of the burnt remains of four individuals, probably an adult male, an adult female, an immature human and an infant (p 227). A burnt flint, a 'slug knife' (SF109) was found to one side of the deposit; a second, a 'knife' or 'spear point' (SF108) on the base slab.

\section{Burial C (fig 51) (Cremation p 228; Stone p 219)}

This cremated deposit was largely contained in a massively built rectangular cist which measured between 0.7 and $0.9 \mathrm{~m}$ long, $0.4 \mathrm{~m}$ broad and $0.35-0.5 \mathrm{~m}$ deep. The cist had been packed in position using small cobbles in a pit a minimum of $\mathrm{c} 1.7 \mathrm{~m}$ long and $1.5 \mathrm{~m}$ broad. The cist was well sealed by a substantial capstone and no soil had penetrated even though a large tree had grown immediately over it. The deposit was spread over most of the bottom of the cist and included a burnt, broken, retouched flint flake (SF110). Five separate deposits of cremated bone, one of them relatively large, were found under the stone packing of the cist. Unfortunately, these deposits and that inside the cist were accidentally mixed after the excavation. This mixed deposit represented parts of the cremated remains of at least eight humans, ranging in age from foetal/neonatal through early and late childhood and adolescence to adults of both sexes. If each of the external deposits represented part of one body then three might have been buried with the cist. However, some of the external deposits may have been multiple.

\section{Burial D (fig 51) (Cremation p 229)}

This cremation deposit lay in a rectangular cist which measured, at the level of the top of the deposit, c $0.5 \mathrm{~m}$ long and $0.2-0.25 \mathrm{~m}$ broad. The cist was set in a subcircular pit between 0.9 and $1 \mathrm{~m}$ in diameter. The lower part of the cist had been backfilled before deposition. The deposit represented parts of the cremated remains of at least seven individuals, apparently varying in age between foetal and adult (p 229). A large flat stone was found approximately $0.3 \mathrm{~m}$ above the top of the cist slabs. It is likely that it was related to the cist and that it was designed to be a capstone. Either the cist was deliberately back-filled and the slab laid on above ( $\mathrm{cf}$ Henge burial $\mathrm{C}$ ) or the slab may have jammed immovably in the pit during an attempt to lower it on to the cist.

\section{Burial E (fig 52) (Bone p 226; Pottery p 213; Stone p 219)}

One of only two inhumations found on the site (with the possible exception of any primary burials in the central enclosure), burial $E$ survived only as a cranial fragment at the $S$ end of a substantial soilfilled cist. The burial, apparently that of a child aged between four and six, or possible even younger, was accompanied by a food vessel (SF41). A distal-end flint scraper (SF110) was also found in the cist fill.

The cist measured a maximum of $0.95 \mathrm{~m}$ long, $0.7 \mathrm{~m}$ broad and $0.5 \mathrm{~m}$ deep. The section of the cist is projected on to the E-W section of the mound (fig $44 \mathrm{C}-\mathrm{D}$ ). There was no base slab but the cist had been sealed, albeit imperfectly, by a large capstone. Because of the massive disturbance of the surface layers of the mound the edge of the pit in which the cist had been set could not be clearly defined. There was clear evidence for the burrowing of small animals, and a root was found growing into the cist. The cist was of irregular construction. The $\mathrm{W}$ side was built using a single slab, as were the $\mathrm{N}$ and $\mathrm{S}$ ends. The $\mathrm{E}$ side, however, was built using a slab much shorter and less broad than that on the $W$. At its $S$ end, in the SE corner of the cist, a long stone with an almost square section had been inserted to fill the gap left by the deficiency of the E slab. The side slabs had been set in narrow slots dug through the old land surface. The inhumation lay on a thin layer of gravel over the OLS. Flags were laid to the E of the cist, over the side slab bringing its level up to that of the $\mathrm{W}$ side. A slab was also laid immediately to the $\mathrm{N}$ end of the cist. The paving may have served a purpose in the burial ritual.

Burial F (fig 52) (Cremation p 230)

This burial, the cremated remains of a young adult female, was contained in a cist cut into the mound 


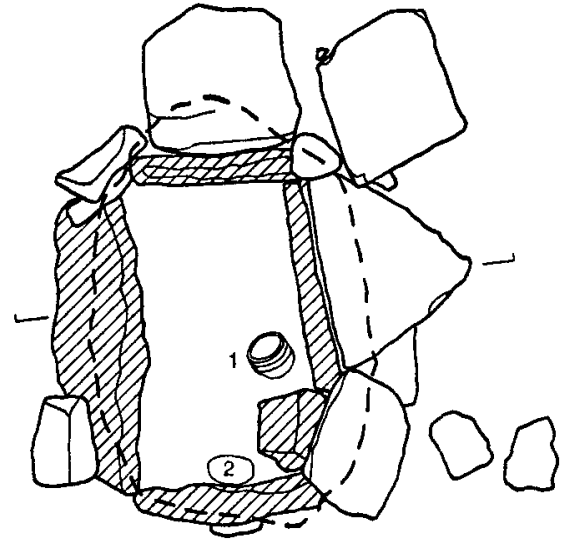

Burial E

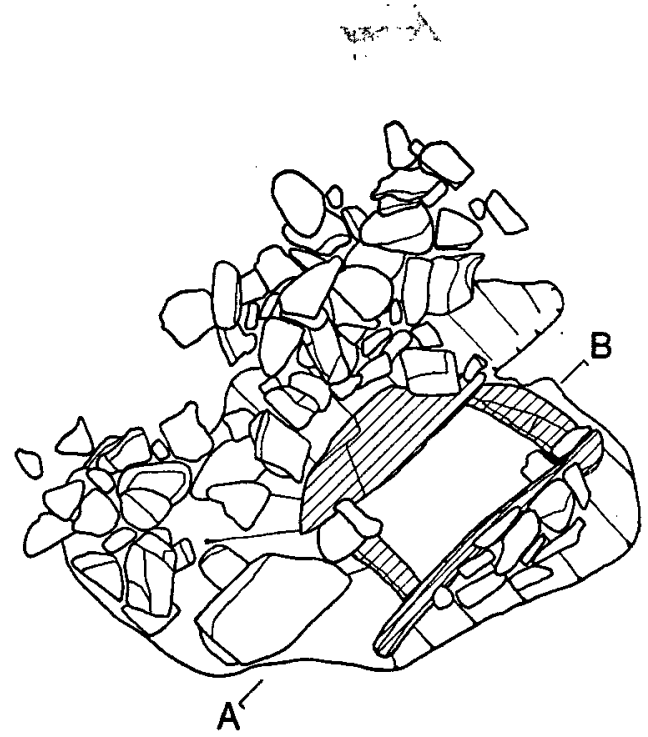

A

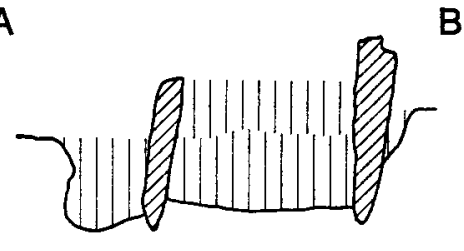

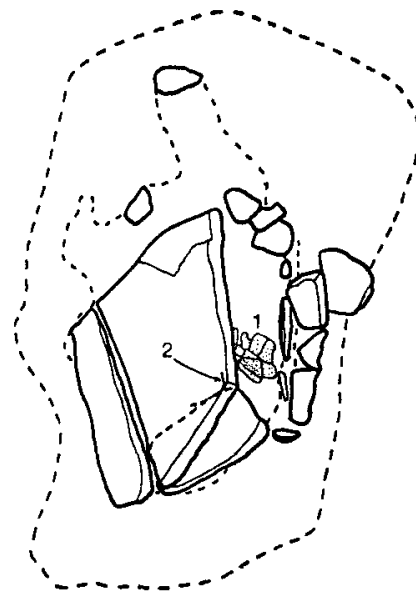

Burial G
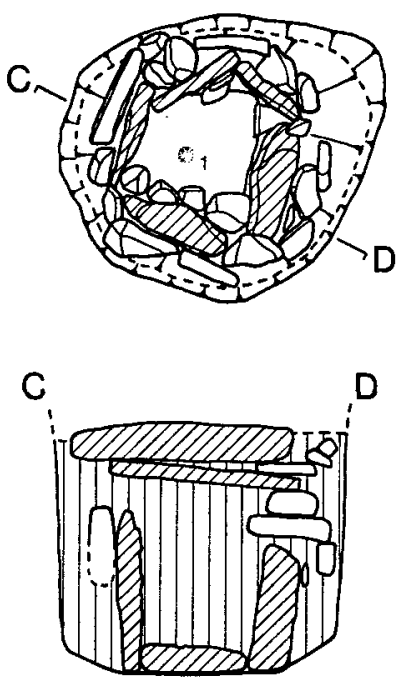

Burial H

\section{Burial $\mathrm{F}$}

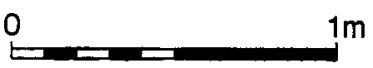

Fig 52 Barrow: Burial E (1 - food vessel SF 41; 2 - cranium); Burial F; Burial G (1 - food vessel SF 42, 2 - jet necklace SF 113); Burial H (1 - cupmark) 
in the SE quadrant. The deposit was mixed with the soil fill of the cist, though whether deliberately or the result of later disturbance was not clear. The cist was found during the machining of a barrow spit; little damage resulted, apart from dislodging the capstone and the further blurring of the edges of the pit or scoop in which the cist was set. The cist was rectangular, measuring a maximum of $0.55 \mathrm{~m}$ long, $0.3 \mathrm{~m}$ broad and $0.55 \mathrm{~m}$ deep. It had no base slab. The sides and ends were built using four slabs. The long sides at NW and SE were both packed in position using smaller stones, on the uphill side apparently using material from the stone capping of the mound (Period III, phase F). The slabs were set into slight grooves cut from the level of the pit bottom.

\section{Burial G (fig 52) (Pottery p 214; Jet necklace p 225)}

The second, presumed, inhumation lay $4 \mathrm{~m}$ to the $\mathrm{N}$ of burial $\mathrm{E}$. It had not been deposited in a cist but had, in part, been covered by a flat slab. The body survived as a scatter of bone crumbs within the severely disturbed outline of a pit measuring approximately $1.7 \mathrm{~m}$ long by $1 \mathrm{~m}$ broad and of uncertain depth. Under the slab remains of a disc-bead jet necklace (SF113, fig 52 Burial G2) were found. In places segments of the necklace had survived in position; in others, gaps had been formed by root and rodent activity, and the beads were scattered. Under the $E$ end of the slab and partly beyond it, parts of a crushed, lugged food vessel were found (fig 52, Burial G, 1; SF42). It has been possible to rebuild part of the vessel.

\section{Burial $H$ (fig 52) (Cremation p 230; Stone p 219)}

Burial $\mathrm{H}$ was set in a polygonal cist, similar in some ways to burial $\mathbf{B}$, and consisted of the cremated remains of at least two adult humans, one male, the other female. The deposit was mixed with the cist fill. The cist measured $0.35 \mathrm{~m}$ in diameter and c $0.4 \mathrm{~m}$ deep. It had a base slab on whose upper surface a clear cupmark was visible (pl 16). The context of the burial was once again unclear because of the later disturbance of the mound's surface. However, the dimensions of the pit in which the cist was set could be determined; it was c $0.6 \mathrm{~m}$ long and $0.45 \mathrm{~m}$ broad at its base and between 0.8 and $0.9 \mathrm{~m}$ in diameter at its highest surviving point, $\mathrm{c} 0.7 \mathrm{~m}$ from the bottom. The capstone was laid on two subsidiary layers of slabs, the upper of which completely sealed the cist; the lower was laid over the edges of the side slabs, leaving a central space.

\section{Burial J (Cremation p 230)}

This cremation deposit, the burial of an adult (female?), lay on a small slab in a shallow amorphous pit in the NE quadrant. The deposit was very severely disturbed.

\section{Burial K (Cremation p 230)}

A light scattering of cremated bone fragments was found within the disturbed part of the stone core of the mound. Denston (p 230) suggests that all the fragments are from one body. It seems likely, therefore, that burial $\mathrm{K}$ was a single cremated body buried in the upper part of the mound and destroyed by the earlier major excavation. The type of burial, whether cisted or not, and its possible association with the cupmarked stone can now never be known.

\section{FINDS}

The nature of the mound has made the assignment of the finds into periods impossible. Apart from those in the burials almost all of the finds were from derived contexts; either the fossil topsoil, the mound material or the fills of the ditch.

It has been possible to arrange the finds in Find Groups by the period in which they were probably deposited and the periods in which they may have originated.

Find Group 1 - Artefacts securely within the natural and man-made features under the old land surface (Period I).

2 - Artefacts within the old land surface, deposited in Periods I, II or early III.

3 - Artefacts on the old land surface and in mixed (root and disturbed) contexts near the edge of the barrow, deposited in Periods I, II, III (A, B) or in the turf element of the later part of $\mathrm{C}$.

4 - Artefacts from mound material deposited during Period IIIB. 
Burials

5 - Artefacts from mound material deposited during IIIC.

6 - Artefacts from material deposited during the completion of the mound (IIIE and F).

Ditch Fills

Miscellaneous

Medieval

\section{THE POTTERY FROM THE BARROW AT NORTH MAINS}

\section{T G Cowie}

\section{INTRODUCTION}

The pottery assemblage from the barrow consists of two substantially complete vessels SF41 and 42, recovered from graves, and some 90 sherds, about 40 fragments and over 100 crumbs most of which are featureless. Many of the sherds and fragments are unrelated pieces, but a sizeable proportion of the upper part of a single vessel (SF21) is represented by 13 of the sherds, 20 of the fragments, and some $20 \%$ of the crumbs.

The published catalogue lists all the identifiable pottery recovered in the course of the excavation, possibly representing over 40 vessels and is set out in the same format as that adopted for the pottery from the henge (see p 155 above).
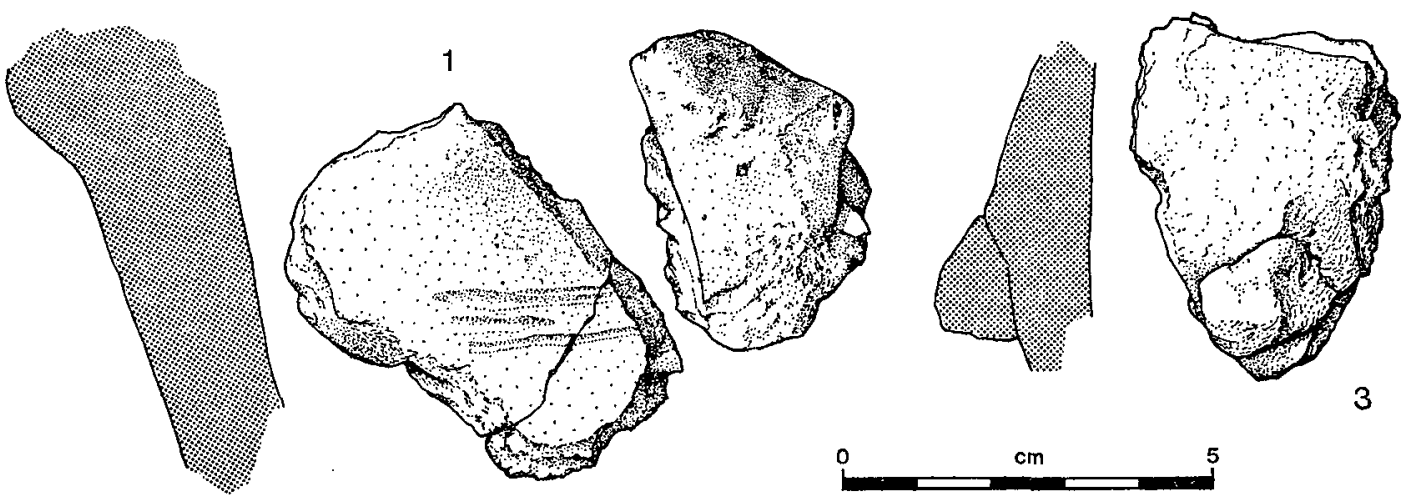

3

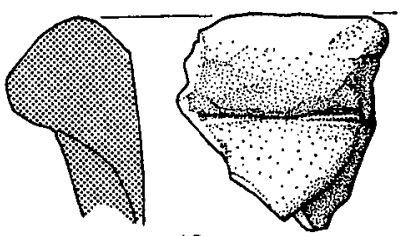

13

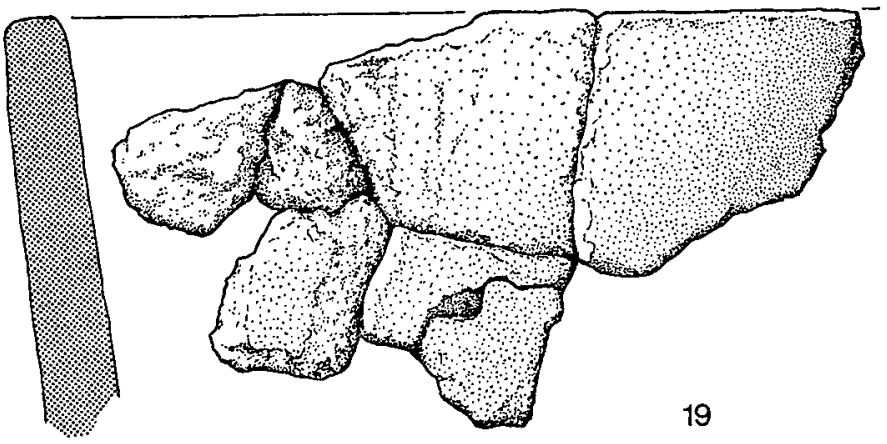

FIG 53 Barrow pottery: miscellaneous sherds, pt 1 


\section{CATALOgue}

\section{Find Group 1}

SF1 (fig 53) Nine body sherds, four fragments and crumbs almost certainly all from the same vessel; one of the sherds is from shoulder carination with a pinched-up rounded lug $\mathrm{c} 12 \mathrm{~mm}$ in diameter lying on a break edge; one of the other sherds may be from near the lug, but overall form of vessel uncertain; light reddish brown; fabric B; on external surface of sherd near lug, two faint grooves c $4 \mathrm{~mm}$ apart; burnt.

SF2 Body sherd, possibly part of same vessel as SF1.

SF3 (fig 53) Body sherd with rounded lug placed on edge of shoulder carination; internal surface very dark grey, exterior brown; fabric B; burnt.

SF4 Two body sherds, two fragments and a crumb probably from same vessel as SF3; doubtful boneend impression on external break edge of one sherd; outside of one sherd badly scorched.

SF5 Two body sherds, almost certainly from the same vessel; fabric B; relatively unabraded.

SF6 Body fragment; fabric C.

SF7 Two body sherds, three fragments and eight crumbs possibly all from shoulder area; fabric C; boneend impression on external surface of one sherd; organic encrustation on internal surface of one sherd; condition variable - fragments burnt, sherds relatively unabraded.

SF8 Two body sherds and a fragment, possibly all part of the same vessel; fabric C.

SF9 Body sherd; fabric C; organic encrustation on internal surface.

SF10 Two joining body sherds and two crumbs; fabric $D$.

SF11 Three body sherds and crumbs, almost certainly all from the same vessel; in two of the sherds, curvature suggests position near rounded shoulder; fabric E; relatively unabraded.

SF12 Body sherd, external surface almost wanting; possibly part of same vessel as SF11.

SF13 (fig 53) Rimsherd from vessel with externally expanding rounded rim; form of vessel and rim diameter indeterminate; reddish brown surfaces, dark grey core; fabric F; abraded. Also a body sherd almost certainly part of the same vessel; relatively unabraded.

\section{Find Group 2}

SF14 Two featureless body fragments and two crumbs; fabric B; doubtful bone or stick impression on external surface of one fragment.

SF15 (fig 53) Tiny abraded fragment of indeterminate position and orientation; internal surface wanting; dark grey and light brown; fabric B (?); very faint traces of parallel grooves or channels with transverse fingernail marks.

SF16 Featureless body sherd and fragment; fabric C; partly burnt after fracture; very abraded.

SF17 Body fragment; fabric D.

SF18 Body sherd; fabric D.

SF19 (fig 53) Six joining sherds, and another five tiny body sherds almost certainly all from the same vessel; form uncertain but possibly from upper part of a simple open bowl with slightly flaring flattened rim; diameter uncertain - c $200 \mathrm{~mm}$; very dark grey surfaces, very dark grey/black core; fabric $\mathrm{E}$; relatively unabraded, but fairly friable; organic encrustation on internal surfaces; (see overall discussion $\mathrm{p} 251$ ).

SF20 Three tiny body sherds possibly from the same vessel as SF19, although these lack encrustation on interior, and possess smoothed external surfaces; relatively unabraded.

\section{Find Group 3}

SF21 (fig 54) Thirteen sherds, 20 fragments and c 20 crumbs - probably all from the same vessel: only portions of the upper part of the vessel appear to be represented; these figures include three rimsherds: the rim is simple and internally bevelled but without any thickening through the addition of clay; approximately $60 \mathrm{~mm}$ from the rim there is a raised moulding corresponding to a major building line. The lower profile is uncertain but it seems reasonable to interpret this moulding as the shoulder of a bipartite (possibly biconical) vessel; rim diameter uncertain - c 260-300 mm. Colour range variable: internal surfaces light brown to dark greyish brown, core light brown, and exterior greyish brown to dark grey; fabric A; decoration: on internal bevel of rim, bone-end impressions applied deeply and at an angle, c 8-9 mm apart; on exterior, all-over finger-pinched 
212 | SOCIETY OF ANTIQUARIES OF SCOTLAND, 1983
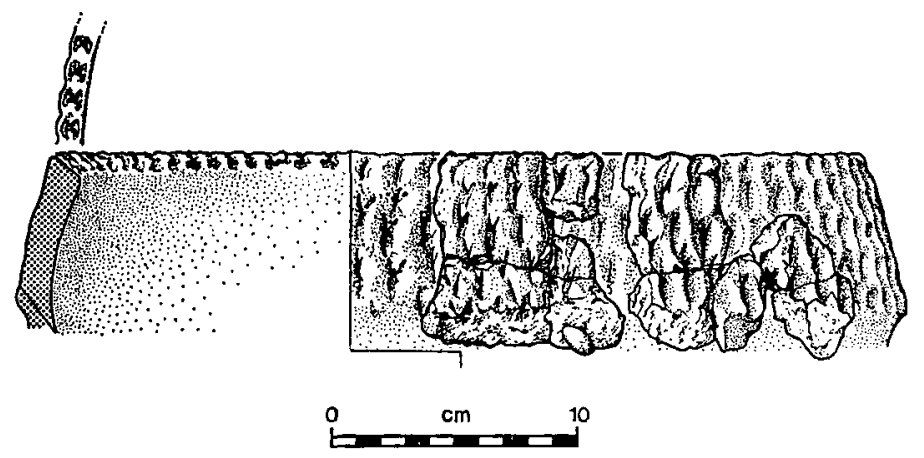

FIG 54 Barrow pottery: late[̨Neolithic vessel SF 21
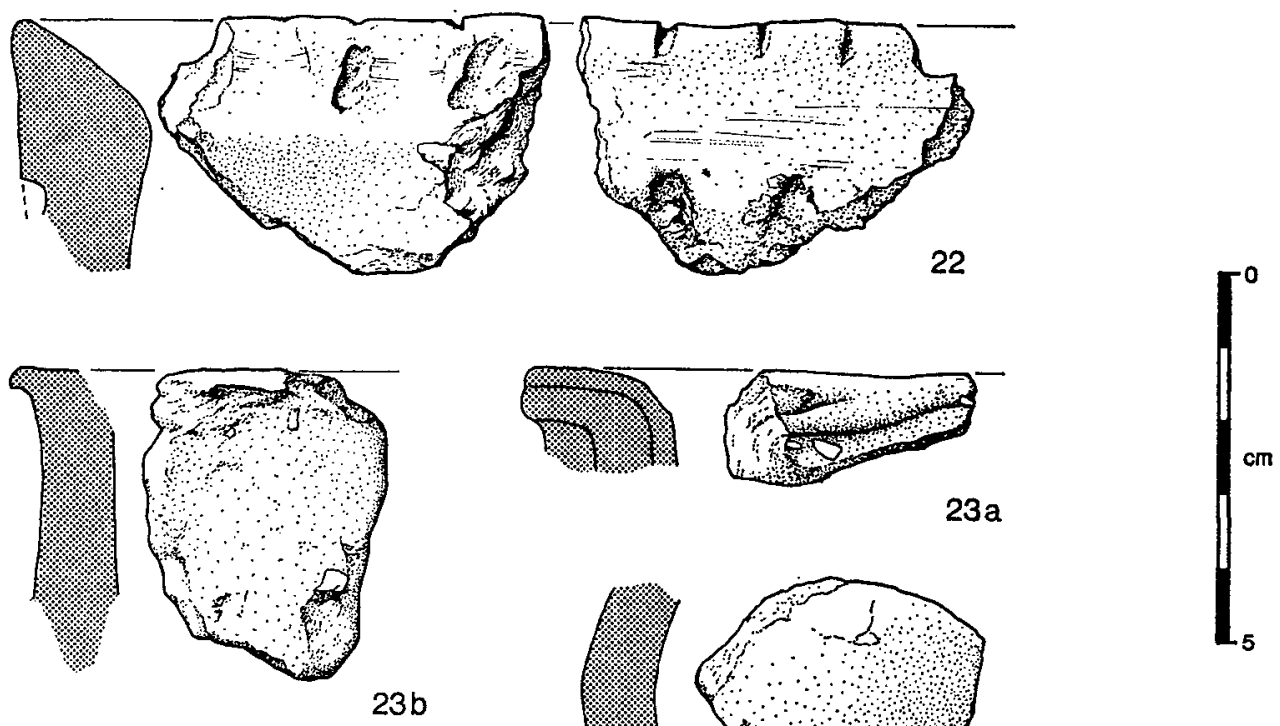

$23 b$
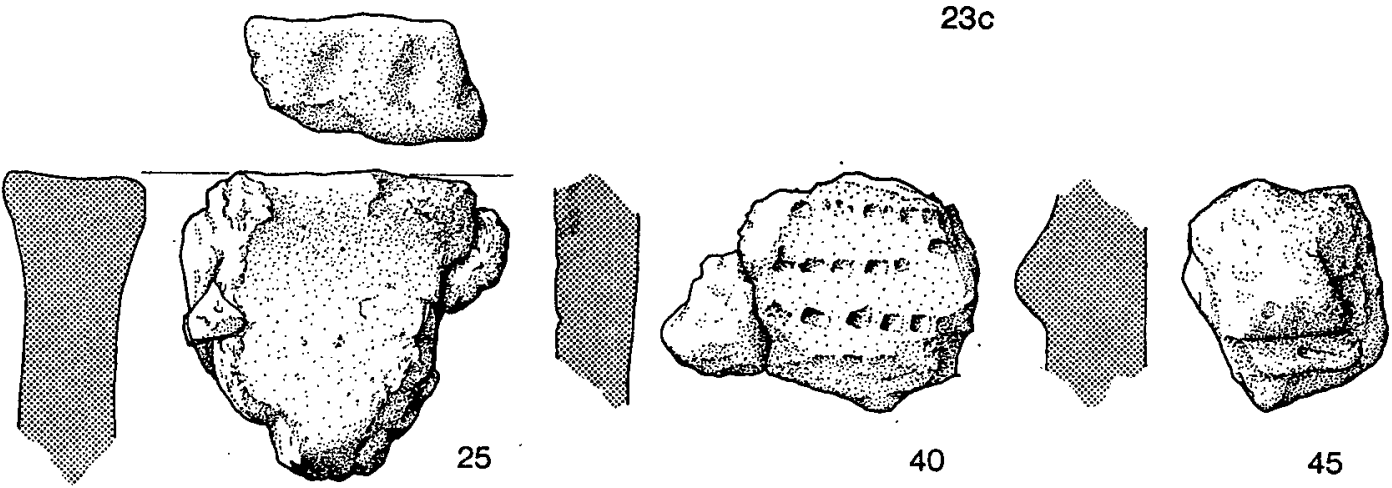

FIG 55 Barrow pottery: miscellaneous sherds, pt 2 
decoration in about four or five rows between rim and 'shoulder' moulding; the individual impressions have been vertically applied, c $9 \mathrm{~mm}$ apart. Sherds relatively unabraded.

SF22 (fig 55) Rimsherd of vessel with narrow fairly steep internal bevel, meeting top of rim at a very acute angle - overall form virtually pointed/triangular; broadly similar rim form to SF21 though more angular; rim diameter uncertain; internal surface pale brown to dark grey; core and exterior dark grey; fabric B; decoration: on internal bevel, traces of two bone-end impressions jabbed in at an angle c $12 \mathrm{~mm}$ apart; on external edge of rim traces of three nicks around lip c $8 \mathrm{~mm}$ apart; c $18 \mathrm{~mm}$ below rim, traces of two deep bone-end impressions but pattern uncertain.

SF23 (fig 55) Three sherds, two fragments and three crumbs; probably all from the same vessel; these include one rimsherd (a) of uncertain form owing to the small size but apparently rounded with a slight external expansion: pressure on the clay during construction of the vessel appears to have resulted in the formation of a slight squeezed-out 'fillet' or lip on the exterior of the rim; one of the other sherds (b) appears to be a 'false rim' from which clay has flaked off but probably from the same vessel. The remaining sherd (c) appears to be a body sherd from the area of the shoulder; rim diameter indeterminate; generally very dark grey; fabric $D$, relatively unabraded.

SF24 Two body sherds, possibly part of same vessel as SF23; relatively unabraded.

\section{Find Group 4}

SF25 (fig 55) Rimsherd of vessel with flattened rim, with slight internal and more pronounced external expansion; straight neck; rim diameter uncertain - c $120 \mathrm{~mm}$; surfaces very pale brown, core very dark grey; fabric C; decoration: on top of rim, shallow transversely set spatula or fingertip impressions; abraded. Also one featureless body sherd and crumbs almost certainly from the same vessel swelling of sherd suggests position just below rim.

SF26 Body sherd and crumb; fabric C.

SF27 Body sherd; fabric C.

SF28 Featureless body fragment; fabric D; very abraded; possibly re-fired after fracture.

SF29 Body sherd; fabric F; burnt after fracture?; relatively unabraded.

SF30 Body sherd; fabric F; relatively unabraded.

\section{Find Group 5}

SF31 Body sherd, fabric C; one possible shallow bone-end or stick impression on external surface; relatively unabraded; organic encrustation on internal surface.

SF32 Two body sherds. The larger of them composed of several joining pieces, almost certainly all from the same vessel; position uncertain but possibly from a flattened or sagging base; diameter indeterminate; fabric $C$.

SF33 Body sherd, apparently re-fired after fracture; possibly from shoulder area; fabric C; abraded prior to re-firing.

SF34 Body sherd; fabric C.

SF35 Base sherd ?, composed of seven joined fragments and crumbs; part of flattened base of indeterminate diameter; fabric $F$.

SF36 Body sherd, fabric F; external surface burnt.

SF37 Body sherd; fabric F; very light fingernail marks on external surface - probably accidental; relatively unabraded.

SF38 Two joining body fragments, wanting internal surface; fabric F; very faint parallel incisions on external surface.

SF39 Two body sherds, possibly from the same vessel; fabric G; relatively unabraded.

\section{Find Group 6}

SF40 (fig 55) Body sherd and joining fragment; brown internal surface, dark grey core, light brown exterior; fabric $\mathrm{C}$; coarse comb impressions on external surface probably applied horizontally remains of four rows and traces of a fifth on break edge.

\section{Burials}

SF41 Burial E (fig 56) Virtually complete food vessel wanting only exterior of base and part of lower body: these areas have flaked or broken off in the past; the vessel is a rather squat ridged vase; the rim is simple and rounded at the lip with a strongly moulded but relatively shallow and narrow 


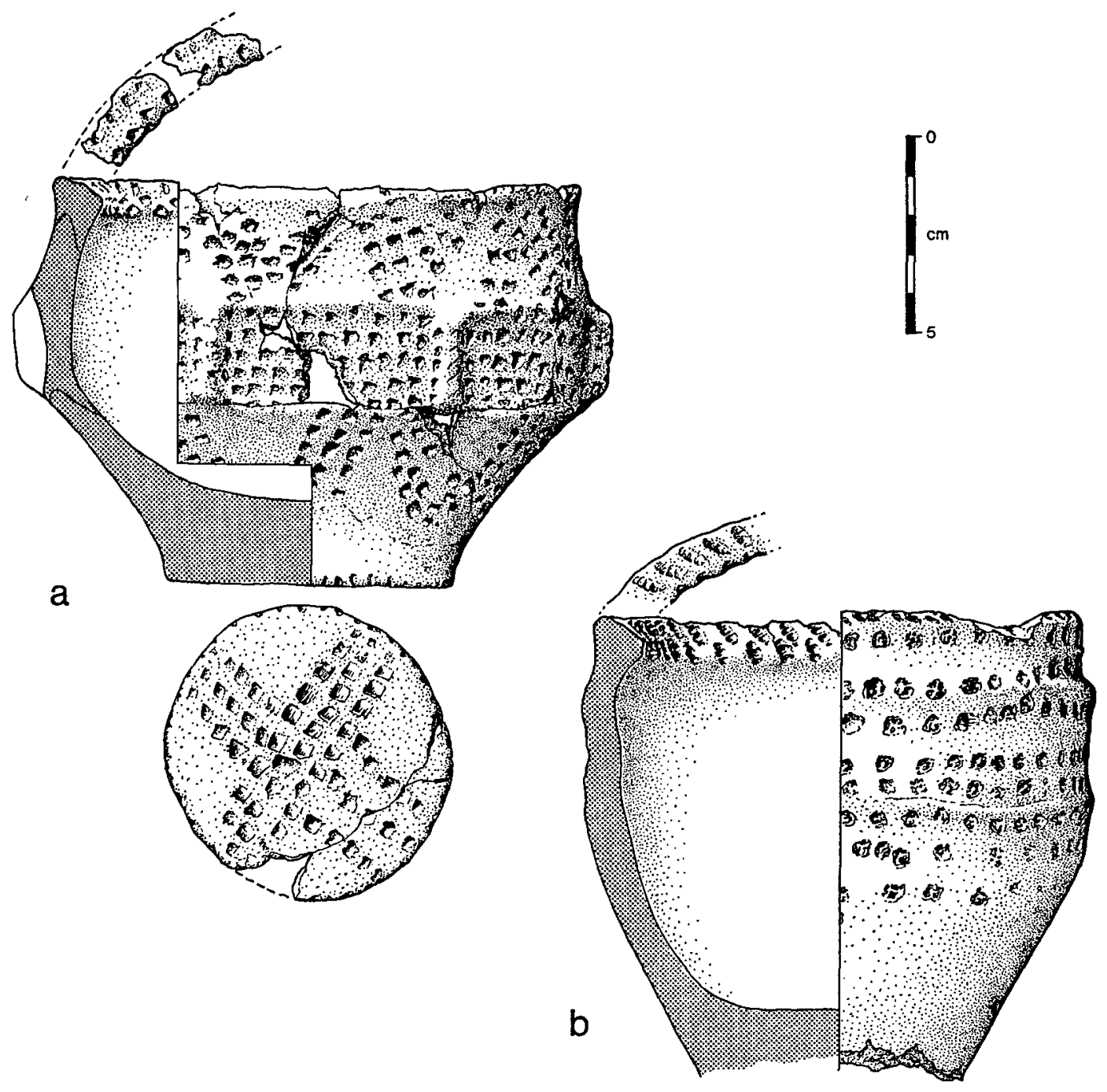

Fig 56 Barrow pottery: food vessels, a - SF 42 ; b - SF 41

internal bevel; externally, there are two raised mouldings on the upper body defining a shallow cavetto zone c $25 \mathrm{~mm}$ wide; below the lower shoulder moulding, the lower body tapers away to what may have been a proportionately substantial base. Surviving height $-120 \mathrm{~mm}$; rim diameter 115-25 mm; wall thickness c 9-10 mm. Interior surface pale brown to dark grey, core dark grey external buff-brownish yellow. Fabric C. Decoration: on internal bevel, vertical and slightly obliquely set whipped cord maggot impressions; on exterior of rim, poorly defined marks, probably bone-end impressions; bone-end impressions have also been applied spaced at 5-10 $\mathrm{mm}$ intervals, or more haphazardly in places, to the neck, cavetto zone and on to the lower body. Virtually complete but surfaces abraded.

SF42 Burial G (fig 56) The greater part of a bipartite vase food vessel, wanting approximately half of the rim and a similar fraction of the body, but with base largely intact; the rim has a proportionately narrow, shallow internal bevel, and a slight external expansion or lip; the neck zone is straight giving way to the upper of two raised mouldings spanned by vertical imperforate stops of which three survive intact c $65 \mathrm{~mm}$ apart, while traces of a fourth stop are present at one break edge; below the 
lower, and quite pronounced, shoulder moulding the body tapers to a substantial upright base $21 \mathrm{~mm}$ thick. The lower moulding corresponds to a well-defined construction line c $47 \mathrm{~mm}$ above the base. Height $-105-10 \mathrm{~mm}$; rim diameter - 130-40 mm; wall thickness $-7 \mathrm{~mm}$. Surfaces pale brown, core dark grey. Fabric C. Decoration: on the internal bevel of the rim, poorly defined boneend impressions probably arranged in two rows around the upper and lower edges of the bevel; on the external lip of the rim, there is a row of bone-end impressions; on the neck the decoration is unclear in places, but appears to consist of roughly triangular areas composed of haphazard boneend impressions with reserved areas in between; the cavetto zone or shoulder groove, and the stops which span it, are completely filled with five rows of carefully applied bone-end impressions; the body is also decorated with bone-end impressions, again arranged in a roughly set out chevron made up of filled and reserved areas; the decoration stops short of the foot of the vessel. The base is ornamented with a saltire pattern composed of bone-end impressions in two bands of three rows, while spaced impressions run around the circumference of the design on the basal angle. Relatively unabraded.

\section{Ditch fills}

SF43 Featureless sherd composed of four joining fragments and crumbs; fabric D; relatively unabraded.

SF44 Tiny abraded sherd, possible part of same vessel as SF43.

SF45 (fig 55) Body sherd with rounded external lug: the lug consists of a small pinched-up boss $\mathrm{c} 10 \mathrm{~mm}$ in diameter protruding from the surface; internal surface and core very dark grey, exterior light brown; fabric D; external surface abraded.

SF46 Body sherd and two fragments almost certainly all from the same vessel; fabric E.

\section{Unstratified}

SF47 Body sherd; fabric C.

SF48 Body sherd; fabric C; possible bone-end impression on external surface.

\section{Medieval}

SF49 One rim, four body and two basal angle sherds of wheel-thrown pottery; gritty cream fabric; two basal angle and one body sherds blackened externally; basal angle sherds turned.

\section{DISCUSSION}

The pottery assemblage from the North Mains barrow for the most part consists of largely featureless sherds differentiable on the basis of their fabric rather than form or decoration, characteristics which are, with only a few exceptions, available for only the small numbers of more complete or better represented vessels. Despite its featurelessness, much of the pottery is nevertheless identifiable, with varying degrees of confidence, as the products of recognizable ceramic traditions. Full discussion of the comparative material will, however, be postponed to facilitate consideration of the pottery from all three North Mains sites as a group, and for the moment only the main features of the assemblage from the mound will be outlined.

\section{Form}

The preceding catalogue lists all the pottery from the barrow and with only a few exceptions the assemblage can be seen to consist of featureless body sherds. For the most part, the position of the sherds on the vessel, and frequently their orientations are uncertain or quite indeterminate. Such featureless material accounts for over $75 \%$ of the whole, but as the sherd assemblage is arguably an almost wholly derived group (see Condition and Context, below), the high proportion of formless body sherds is perhaps not surprising. Discounting the more complete vessels, SF41 and 42, found in graves, pieces possessing recognizable formal features only include the following: rimsherds - SF13, possibly SF19, 21-23, 25; body sherds with lugs - SF1, 3, 45, and base sherds SF32 and 35.

Inadequate information is available for the reconstruction, with any certainty, of the profiles of the vessels represented by SF13 and 23, but it seems likely that both are variations on Neolithic bowl forms. SF23 may have been a shouldered bowl, but no accurate reconstruction is possible for the other vessel. SF19 consists of an ambiguous group of consolidated sherds discussed more fully presently ( $\mathrm{p} 251$ ). The three lugged sherds would not be out of place in the same 'Western Neolithic' bowl potting tradition. 
The pottery grouped as SF21 represents portions of the upper part of what appears to have been a substantial bipartite vessel, with good parallels in later Neolithic decorated pottery (see discussion p 253 below). It seems likely that the single rimsherd, SF22, derives from a similar vessel, but this cannot be proven. The overall form of the vessel represented by the single rimsherd SF25 is unfortunately indeterminate but the rim form again finds closest parallels among late Neolithic and early Bronze Age pottery, specifically among the northern food vessels, which are, in any case, represented in the pottery assemblage from the mound by the more complete vessels SF41 and SF42 (see discussion p 255 below).

In a number of instances it is possible to suggest, with varying degrees of certainty, that some of the otherwise featureless sherds may be from the same vessel but in hardly any case does this assist in the reconstruction of the form and the suggested minimum number of some 40 prehistoric vessels in the assemblage must therefore be taken as no more that an extremely cautious estimate. The fabric analysis summary is presented on $\mathrm{p} 257$.

\section{Decoration}

Few of the sherds are decorated - not unexpectedly perhaps in view of the interpretation of much of the assemblage as products of the 'Western Neolithic' potting tradition - and the following list covers all the instances of decoration or possible decoration noted in the assemblage: bone-end impression - SF4 (dubious), 7, 14 (dubious), 21, 22, 31 (dubious), 41, 42; comb impressions - SF40; spatula/fingertip SF25; finger-pinching - SF21; fingernail impressions - SF15 (tiny fragment) and 37 (dubious); incisions SF38 (dubious); and maggot impressions - SF41.

In several instances, noted above, the identification is dubious and the sherds concerned have been discounted from further consideration. Although indistinguishable in fabric from the finer pottery in the group, the single body sherd SF40 is of interest because of the presence of coarse comb decoration. If not a typically beaker fabric, the sherd may at least indicate the influence of beaker pottery (but the possible significance of this will be discussed presently). Some interest attaches also to the surviving fragments of the bipartite vessel represented by SF21, with all-over (?) rusticated decoration produced by finger-pinching the surface of the vessel, a distinctive technique matched closely in vessels of broadly similar form from Brackmont Mill, Fife (Longworth 1967) (see discussion p 253 below). The external decoration of the vessel represented by the single rimsherd SF22 is uncertain. The decoration of the internal bevel has been applied with the epiphysis of a small bone, and such bone-end impressions form part of the repertoire of impressed decorative techniques introduced by later Neolithic potters and subsequently maintained in the derivative potting traditions of the earlier second millennium.

The food vessels, SF41 and 42, both possess decorative schemes made up of bone epiphysis impressions, applied in a variety of ways and with varying degrees of care to produce quite distinctive patterns. On one of these, 'maggot' impressions have been applied to the internal rim bevel, while a more spatulate implement may have been used to decorate the rim of the vessel also interpreted as a possible food vessel but unfortunately represented by only the single rimsherd SF25, and a further, uninformative, fragment.

\section{Condition, size and context}

The ceramic assemblage recovered from the mound totalled some 230 pieces, ranging from indeterminate crumbs to complete vessels, and some consideration of the surviving condition and context of discovery of the pottery is relevant to the present discussion.

Only three vessels are represented in sufficient quantity to suggest that their context represents the site of their deliberate deposition as more or less complete pots. These are the food vessels SF41 and 42, the latter incomplete, recovered from graves, and the fragmentary vessel SF21. Although lacking much of the upper part and all of the lower body, SF21 was recovered from an ambiguous context near the very edge of the mound, where loss of portions through post-deposition damage cannot be ruled out.

With these exceptions the remainder of the assemblage mostly consists of small sherds, fragments and crumbs often recovered from discrete, though not necessarily widely removed, locations, and rarely interpretable with any certainty as parts of the same vessel. The following catalogue numbers include pieces from different findspots thought, with varying degrees of certainty to represent parts of the same vessel: SF1 $+2 ; 3+4 ; 5 ; 7 ; 8 ; 11+12 ; 13 ; 23+24 ; 25 ; 32 ; 39$. In considering this list of possible related sherds, two points must be emphasized. First, the groups of material catalogued together in this way are largely composed of featureless body sherds and fragments, and therefore have to be treated with due caution; However, they were used in assessing the minimum number of vessels in the assemblage. Second, it must be stressed that although some of these groups are composed of a number of pieces, they would 
hardly constitute significant portions of the vessels represented, even if they could be proved to derive from the same vessel. The majority of the sherds in the assemblage are less than 30 by $30 \mathrm{~mm}$ in size and are frequently more or less abraded. Together, these factors favour interpretation of the bulk of the assemblage as derived fragmentary ceramic material lying scattered on the old ground surface or in the fossil subsoil and subsequently sealed by or incorporated into the mound at the various stages of its construction. Such an interpretation could account for the presence of all the 'Western Neolithic' pottery in the assemblage, although it leaves unanswered the question of the original context of this material. In view of the pre-barrow stratigraphy, however, it might be reasonable to invoke an explanation in terms of dispersal of household pottery with other domestic debris into a cultivated area.

The two sherds, SF25, interpreted as parts of a single food vessel, were recovered from a Period III, phase $\mathrm{A}$ context and are significant in that they may provide an approximate terminus post quem (in ceramic terms) for this stage of construction of the mound, in so far as they indicate that such pottery may have been in currency by then. In view of the relatively small size of the whole assemblage, however, the absence of such pottery in the stratigraphically earlier deposits could just be fortuitous. In this context, a tiny fragment - really no more than a sliver - of decorated pottery, SF15, retrieved from a Period I deposit, is worth mention as a possible fragment of beaker, but its tiny size makes this interpretation tentative (to say the least). Beaker pottery may also be represented by a solitary sherd (SF40) recovered from a stratigraphically late context (Period III, phase E), possibly best interpreted as a further example of accidental incorporation into the mound of pottery in an already fragmentary condition. Finally, the group of medieval material (SF49) is worth mention: possibly representing the remains of only one vessel (see below) the sherds in question were recovered from the upper ditch fill, from the topsoil and stone core of the mound - all contexts where their presence can be readily accounted for in terms of medieval or later deposition, or disturbance.

\section{Medieval pottery from the barrow}

Mr William Lindsay, of the Scottish Urban Archaeology Unit, has kindly contributed the following note on the seven small sherds of wheel-thrown medieval pottery recovered from the barrow:

All are in a cream fabric with sub-angular quartz grits up to $0.5 \mathrm{~mm}$ in length. Black, white and orange to brown inclusions are also present. The fabric of six of the pieces is remarkably soft but it may be a variant of the white gritty fabrics so common on the east coast of Scotland, the softness perhaps being caused by the nature of the deposits at Strathallan. One rim and two body sherds are probably from an unglazed jug or jugs, while one body and two basal angle sherds are smokeblackened externally and may be parts of a cooking pot. However, the seven sherds could equally be parts of one unglazed jug the base of which has been smoke-blackened. The sherds are too fragmentary to give any indication of form, and no accurate date can be given for their manufacture.

\section{THE FLAKED STONE ASSEMBLAGE FROM THE BARROW AT NORTH MAINS}

\section{R Wickham-Jones}

\section{DESCRIPTION}

With the exception of four flints, all of the 62 pieces in the collection were recovered from the general matrix of the mound. Every piece is the result, if only the unintentional by-product, of flaking stone and 20 of these flakes, SF53 and 54, 72-74 and 96-110, show additional flaking to alter the shape or consolidate an edge for use. As well as flaking, alteration by polishing is represented amongst the collection, on SF106, but cannot be demonstrated to have taken place on site.

\section{Materials}

Forty-four of the pieces are flint, nine are pitchstone, seven quartz and two mudstone. Though the pitchstone is only present in small quantities, the débitage, eg SF50, 65, 69 and 71, argues for some knapping on site. The regular aspect of the small assemblage of quartz on this site would suggest that quartz also was used to supplement flint when necessary. The presence of both a retouched flake of mudstone and an unaltered one indicate some use on site. The polishing over the dorsal surface of the retouched flake, 106 , suggests that it was originally part of a polished stone axe in which form it was probably first intro- 
duced into the assemblage from elsewhere. When the axe broke it is possible that the fragments were flaked into smaller, useful tools, as the stone, when hard enough, may be knapped like flint. However, it is also possible that the two flakes were products of the breakage itself and are not man-made but were recovered and later re-used. There is no other evidence for the use of polished stone axes upon the site and, as most of this axe is missing, other such tools cannot be inferred with certainty. This use of mudstone, whether naturally or artificially flaked, adds to the picture already noted, of an assemblage composed predominantly of flint to which other stones could be added when suitable.

Apart from the flint (see $\mathrm{p}$ 258) the other stones may have been collected from the same gravel source although the mudstone may have been brought, as an axe, over a greater distance. The pitchstone, however was derived from Arran (Thorpe \& Thorpe, infra p 259).

TABLE 7

Cat No

Period Groups 1-3

SF50 Secondary chunk (pitchstone)

SF51-2 Inner flakes (52 quartz)

SF53 (fig 57) Retouched piece. Inner flake; grey; corticated; broken; segment surviving; triangular plan; straight left side forming pointed proximal with slightly convex right side; broad slightly concave distal; irregular bifacial edge retouch on right side and in distal concavity where it emphasizes the tip at the distal end of the right side; $25: 22: 02 ; 127^{\circ} ; \mathrm{r} 54^{\circ} ; \mathrm{d} 32^{\circ}$; single edge retouched point; (lop sided, oblique arrowhead)

SF54 (fig 57) Retouched piece. Inner flake; dark grey; lightly corticated; lightly patinated; artificial platform; medium hammer; irregularities of shape caused by flaws and mis-hits; long steep retouch on curved distal end; $53: 34: 14 ; \mathrm{d} 59^{\circ}$; scraper flake

\section{Period Group 4}

SF55-6 Inner flakes (both quartz)

SF57 (fig 57) Irregular blade

Period Group 5

$\begin{array}{ll}\text { SF58 } & \\ \text { SF59 } & \\ \text { SF60 } & \\ \text { SF61-3 } & \\ \text { SF64-71 } & \\ \text { SF72 } & \end{array}$

Inner chunk (quartz)

Secondary chunk (quartz)

Inner chip

Primary flakes

Inner flakes $(65,69,71$ pitchstone; 66 burnt)

(fig 57) Retouched pieces. Inner flake; dark translucent grey; lightly patinated; very irregular shape; large irregular retouch on left edge; $38: 35: 07 ; 160^{\circ}$; single edge retouched piece

SF73

(fig 57) Retouched piece. Inner flake; cream-yellow; corticated; lightly patinated, narrow blunt proximal; convex sides and distal; long thin invasive retouch around distal and right side; small edge retouch on left edge; $33: 27: 06 ; 148^{\circ} ; \mathrm{r} 52^{\circ} ; \mathrm{d} 68^{\circ}$; distal and side scraper

SF74 (fig 57) Retouched piece. Inner flake; burnt; dark grey; lightly corticated; patinated; broken; segment surviving; irregular plan; remnant retouch on distal end; macroscopic edge damage on distal end; $12: 13: 05 ; \mathrm{d} 79^{\circ}$; broken end retouched piece

Period Group 6

SF75 (fig 57) Core trimming flake

SF76 Secondary chunk (pitchstone)

SF77 Secondary chip

SF78-79 Inner chips

SF80-84 Secondary flakes (84 pitchstone)

SF85-95 Inner flakes (86 mudstone; 92, 93, 95 pitchstone; 89 quartz; 91 burnt)

SF96 (fig 58) Retouched piece. Secondary flake; translucent grey; slightly corticated; lightly patinated; artificial platform; hard hammer; straight proximal; convex left and right sides curving to blunt distal; large steep retouch on distal end of left side; macroscopic edge damage on dorsal face under retouch and on ventral and dorsal faces of right edge; $60: 45: 15 ; 185^{\circ} ; \mathrm{r} 30^{\circ} ;$ scraper flake (fig 58) Retouched piece. Secondary flake; grey corticated; lightly patinated; artificial platform; hard hammer; small straight proximal; convex left and right sides curving to blunt convex distal; large steep irregular retouch on distal end of left side and around distal; macroscopic edge damage on dorsal face under retouch and on ventral and dorsal faces of right edge; 67:46:16; $161^{\circ} ; \mathrm{r} 38^{\circ} ; \mathrm{d} 67^{\circ}$; scraper flake 
TABLE 7 (continued)

Cat No

SF98

SF99

SF100

SF101

SF102

SF103

SF104

SF105

SF106 (fig 59) Polished retouched piece. Mudstone; inner flake; green/grey; artificial platform; straight

(fig 58) Retouched piece. Secondary flake; grey; partially corticated; patinated; artificial platform; soft hammer; straight proximal; straight sides; blunt curved distal; large invasive retouch over left side and distal; macroscopic edge damage on dorsal face under retouch and on ventral and dorsal faces of right edge; $52: 36: 15 ; 159^{\circ} ; \mathrm{r} 45^{\circ} ; \mathrm{d} 65^{\circ}$; scraper flake

(fig 58) Retouched piece. Inner flake; grey; corticated; artificial platform; hard hammer; straight proximal and right sides; convex left side and distal; steep irregular retouch around distal; $20: 23: 10 ; 156^{\circ} ; \mathrm{r} 104^{\circ} ; \mathrm{d} 71^{\circ}$; distal end scraper

(fig 58) Retouched piece. Secondary flake; burnt; grey; corticated; lightly patinated; broken; left side surviving; natural platform; irregular plan; steep remnant retouch on straight distal; 18:13:07; $\mathrm{d} 71^{\circ}$; broken distal end scraper

(fig 58) Retouched piece. Inner flake; cream; corticated; broken; segment surviving; irregular plan; small irregular edge retouch on straight left side; macroscopic edge damage on dorsal face under retouch and around proximal and right edges and on the ventral face of the distal end right side; $17: 16: 05 ; 166^{\circ} ; \mathrm{r} 64^{\circ} ; \mathrm{d} 79^{\circ} ; \mathrm{p} 70^{\circ}$; broken single edge retouched piece

(fig 58) Retouched piece. Inner flake; dark grey; slightly corticated; patinated; broken; distal segment surviving; hinge ended; straight sides and ends; small irregular invasive bifacial retouch on right side; macroscopic edge damage under retouch and on proximal edge; $11: 14: 05 ; \mathrm{r} \mathrm{48}{ }^{\circ}$; p $75^{\circ}$; broken bifacially edge retouched piece

(fig 59) Retouched piece. Inner flake; grey; corticated; lightly patinated; broken; segment surviving; straight proximal left and distal; slightly concave right side; small irregular bifacial edge retouch on right side; shallow invasive irregular retouch on distal end of left side; 12:12:04; $191^{\circ} ; \mathrm{r} 51^{\circ}$; broken retouched piece

SF104

(fig 59) Retouched piece. Inner flake; pink; corticated; patinated; broken; segment surviving; irregular plan; pointed proximal; convex left side; concave right side; straight distal; small irregular edge retouch around proximal left and right edges; $14: 05: 03 ; 171^{\circ} ; \mathrm{r} 67^{\circ} ; \mathrm{p} 50^{\circ}$; broken miscellaneous retouched piece

SF105

(fig 59) Retouched piece. Inner flake; dark grey; partially corticated; patinated; broken; proximal surviving; straight sides; trapezoidal plan, small irregular edge retouch on right edge and distal emphasizing point between distal and right sides; macroscopic edge damage on left side; $10: 13: 04 ; 170^{\circ} ; \mathrm{r} 67^{\circ} ; \mathrm{d} 65^{\circ}$; miscellaneous retouched piece; (piercer) proximal; blunt convex distal; straight left and right sides; dorsal face polished into three longitudinal facets by groups of short parallel striations running across the fiake; small irregular edge

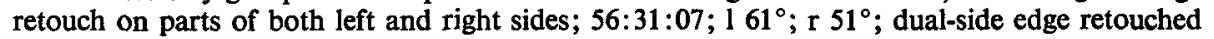
piece; (re-used flake from polished axe)

BURIALS

Burial B

SF107

(fig 59) Retouched piece. Inner flake; burnt; white; corticated; artificial platform; blunt proximal; slightly convex sides curving to pointed distal; large invasive retouch over all of dorsal surface and most of ventral; $75: 33: 10 ; 152^{\circ} ; \mathrm{r} 60^{\circ} ; \mathrm{d} 66^{\circ} ; \mathrm{p} \mathrm{50}$; bifacially retouched leaf shape; (knife, spear point)

SF108

(fig 59) Retouched piece. Inner flake; burnt; white; corticated; pointed proximal and distal; convex right; straight left; small invasive retouch over all dorsal surface; $43: 17: 08 ; 160^{\circ} ;$ r $55^{\circ}$; unifacially retouched leaf shape; (slug knife)

Burial $C$

SF109

(fig 59) Retouched piece. Inner flake; burnt; white; corticated; broken; segment surviving;

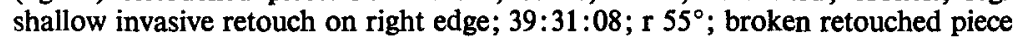

Burial E

SF110

(fig 59) Retouched piece. Secondary flake; dark grey; slightly corticated; lightly patinated; blunt proximal; straight sides; convex distal; steep invasive retouch around distal; irregular invasive retouch on ventral surface at proximal end to thin bulb of force; $20: 16: 07 ; 164^{\circ} ; \mathrm{r} 66^{\circ} ; \mathrm{d} 53^{\circ}$ p $4^{\circ}$; distal end scraper 

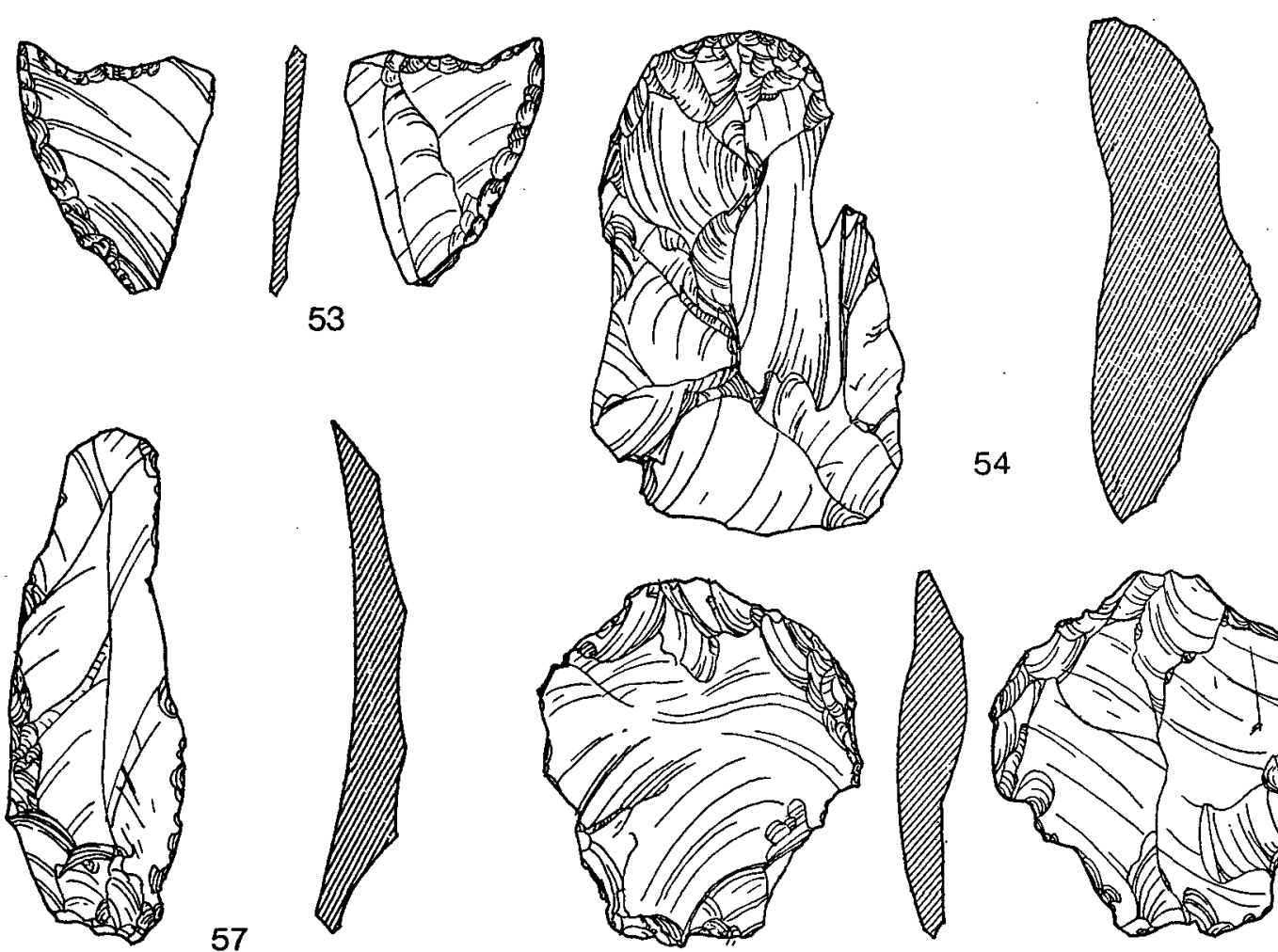

57
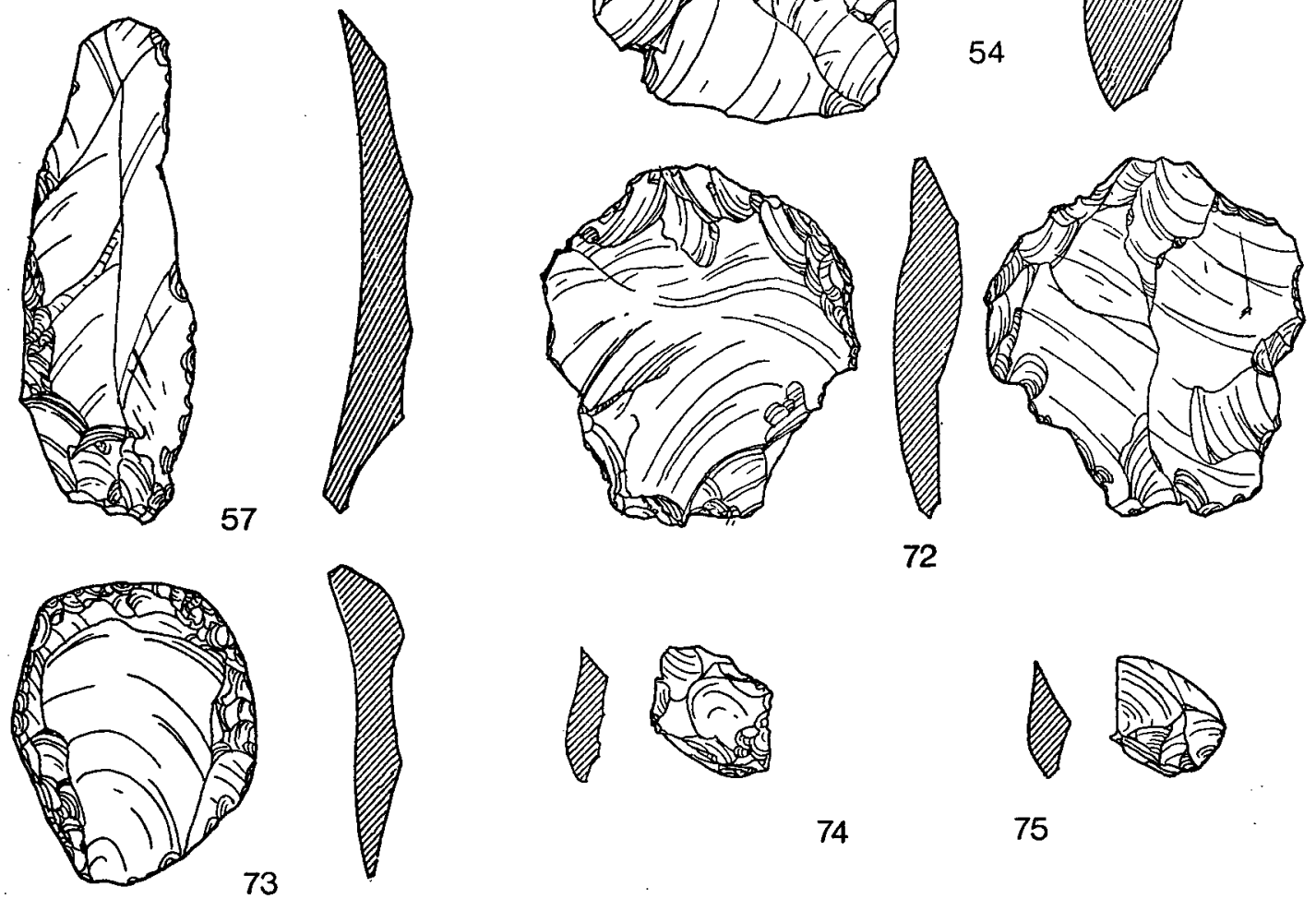

72
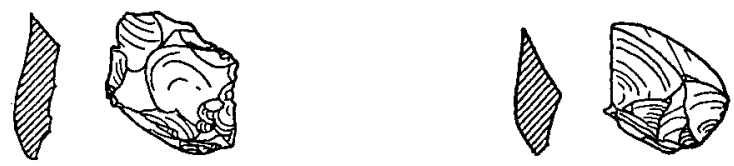

74

75

Fio 57 Barrow flaked stone, pt 1 (scale 1:1)

No unflaked pebbles of flint survive to allow an examination of the size and quality of the raw material in use. Some of the flakes are large, suggesting larger pebbles and an overall examination of the assemblage indicates that the flint was of good quality. There are a few instances where the knapping has been affected by isolated flaws, SF54 for example, but in general an homogeneous material is indicated. catalogue.

The states of both post-deposition cortication and patination have been noted throughout the

\section{Technology: I primary knapping}

The small size of the collection makes it very unlikely that knapping took place on site. However, examination of the pieces reveals some details of the methods used in the creation of the assemblage. Subsequent alteration and breakage has reduced the number of flakes from which such information may be inferred but, for example, a preference for the use of artificial platforms upon which to strike may be seen. Of the 16 flakes preserving their platforms only on one had a natural, slightly rough, cortex surface 
been used. On 15 the removal of a flake had created an artificially flat surface which on two was further refined by the formation of small facets to strengthen the edge and aid the production of regular flakes.

From the 16 flakes preserving detail of detachment characteristics (see $p$ 164) a preference for the use of medium hammers such as bone or antler was noted, although both hard and soft had also been used. On one of those struck by a softer hammer there were indications of indirect percussion through a punch but the rest were most probably struck with direct percussion.
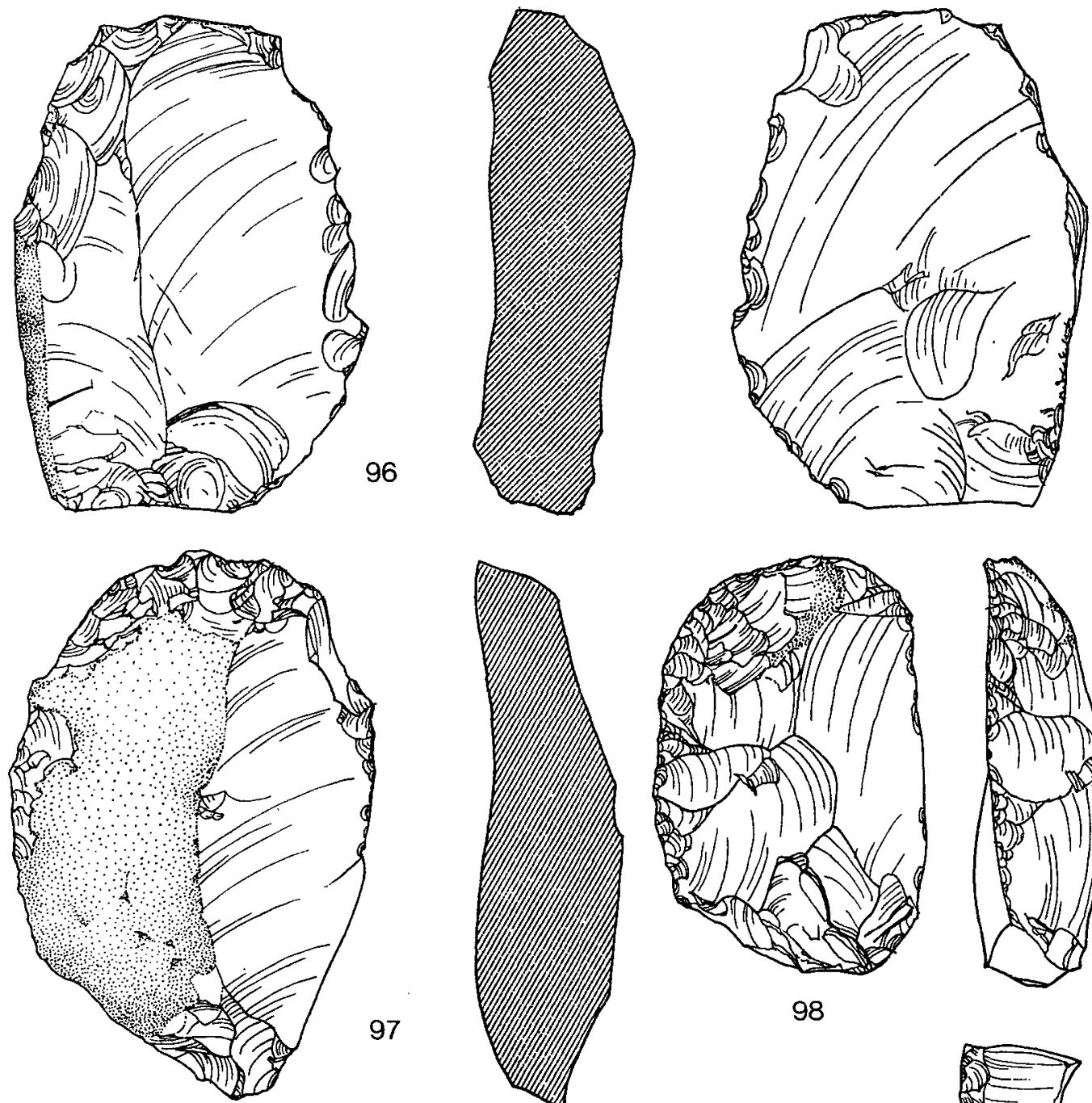

97
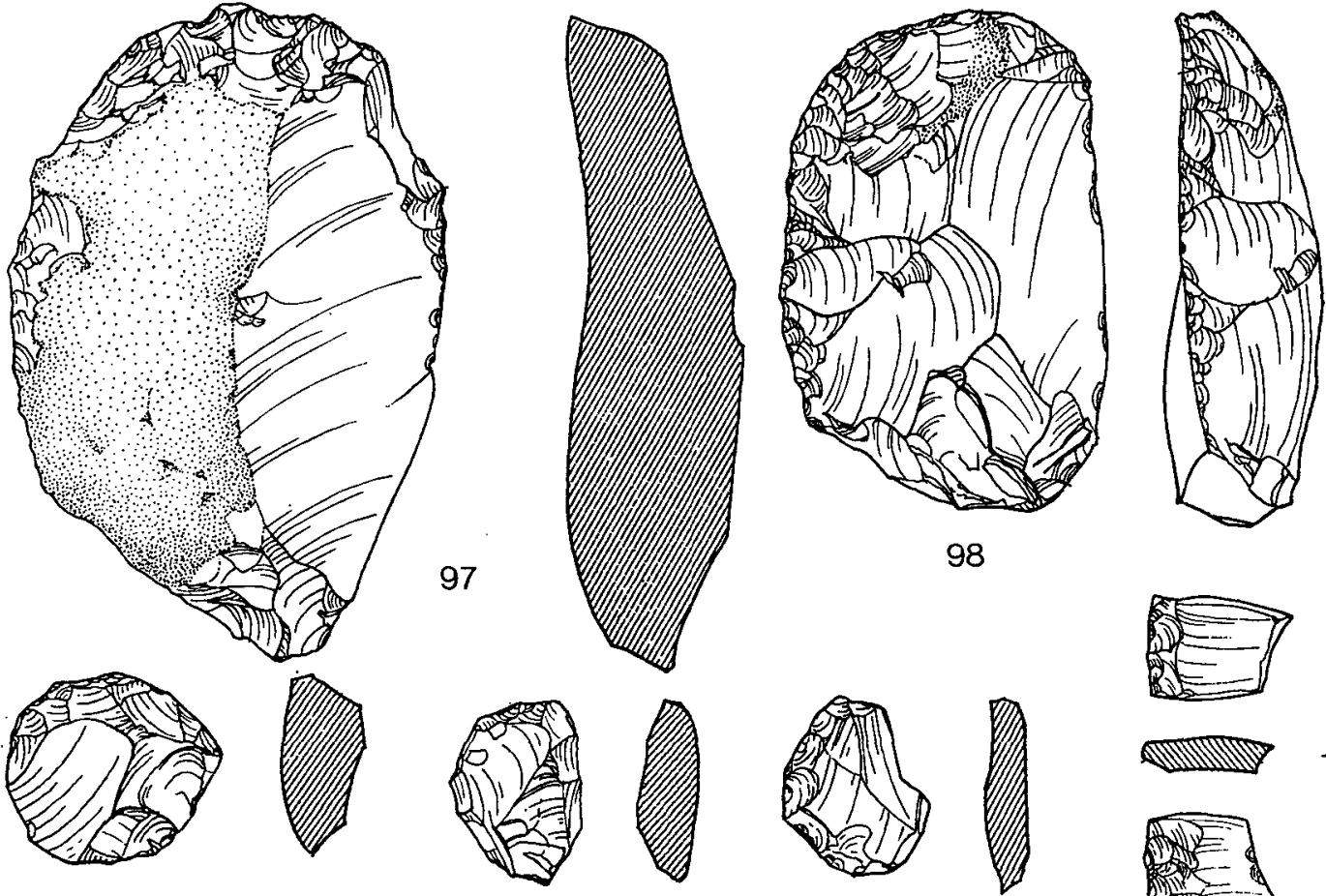

99

100

101

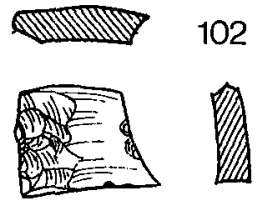

Fig 58 Barrow flaked stone, pt 2 (scale 1:1) 
222 | SOCIETY OF ANTIQUARIES OF SCOTLAND, 1983
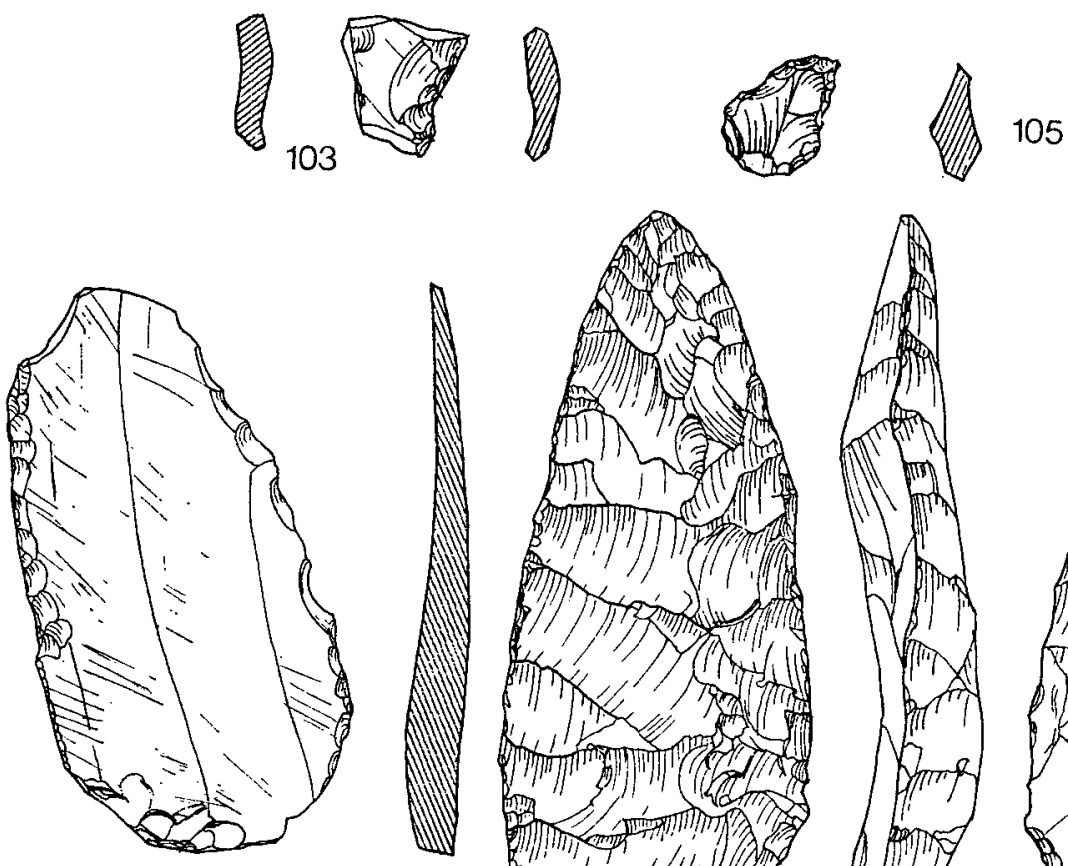

106
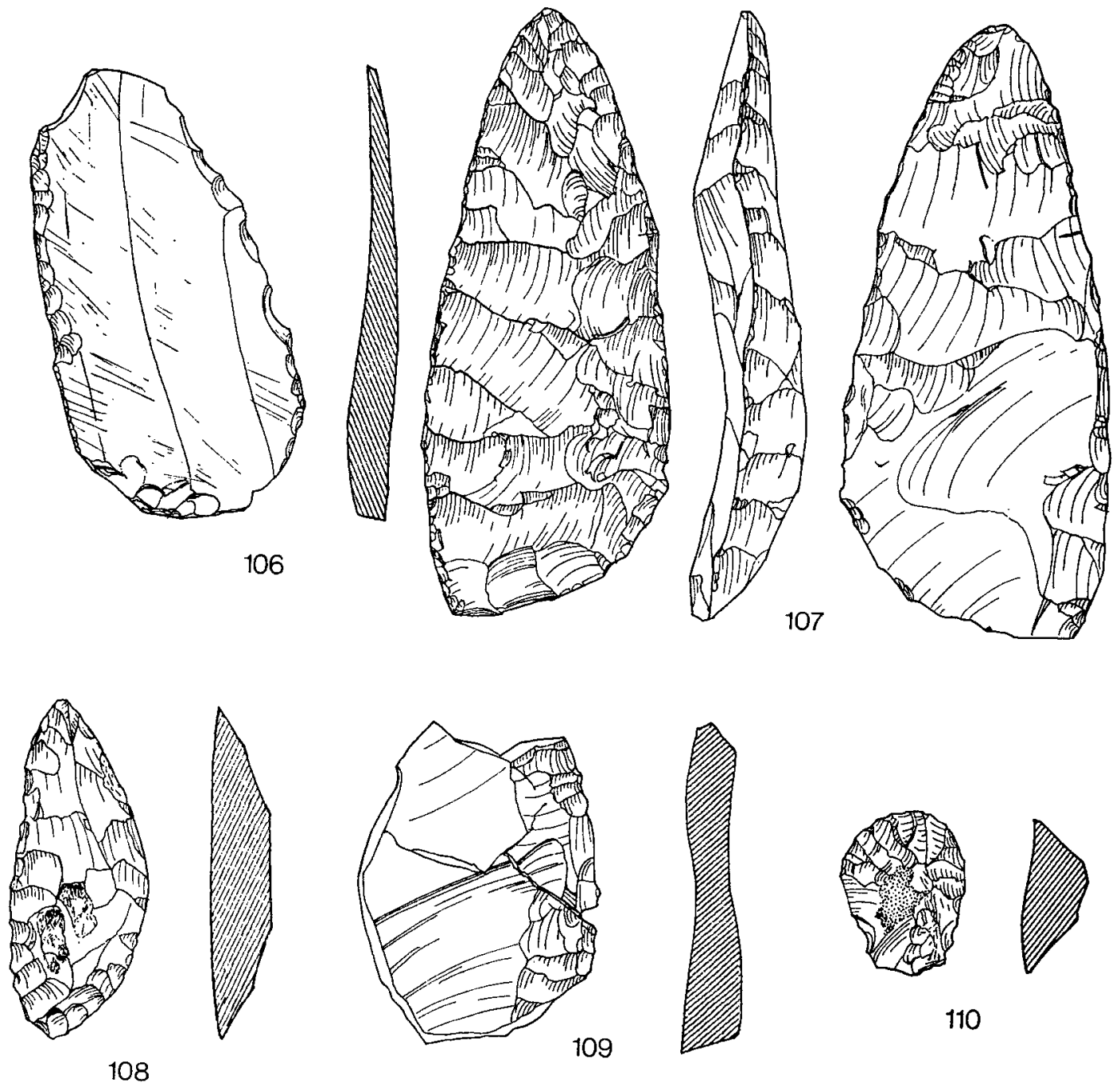

110

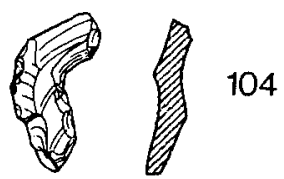

FIg 59 Barrow flaked stone, pt 3 (scale 1:1) 
There is quite a high incidence of hinge fracture at the distal end of the flakes where lack of sufficient force in the blow has caused the flake to terminate abruptly in a rounded surface as the ventral face curves towards the dorsal side. A variety of causes may contribute towards this lack of force such as the use of too soft a hammer, too little strength on the part of the knapper, or the 'give' that inevitably occurs when a hand-held core is struck. Such terminations, however, do not necessarily reduce the usefulness of otherwise regularly shaped flakes which may be employed either as they are or altered by secondary knapping.

\section{Technology: II secondary knapping}

The stoneworkers used various techniques of retouching, to achieve two different results (supra p 164). Three different types of retouching exist amongst the 19 retouched pieces of flint. Many, for example SF73, 53 and 102 etc, have stretches of small scars which are sometimes long and thin but more often irregularly shaped. These appear to have been used both to create particular edges and to shape the flake. The use of pressure flaking with a tool such as an antler tine will quickly produce retouch such as this although other methods are possible. SF96, 97 and 72 have larger steeper scars that are wide in relation to their length and show marked ripples.

Two pieces from burial B are badly burnt. Enough remains of both SF107 and 108 for an examination to indicate that they differ in technology and morphology from the rest of the assemblage. SF107 has been thinned and shaped by the removal of large regular flakes across both surfaces. It is likely that this was formed by direct percussion upon the flake edge with a baton of antler or wood. SF108 is much smaller, the ventral surface has been left untouched and the dorsal face preserves a much steeper profile as less thinning has taken place. Although the piece could have been shaped with an antler baton and direct percussion like SF107 it would also be possible to use pressure-based retouch. The burnt piece from burial C, SF109, is too greatly damaged for any detail to be observed. The piece from burial E, SF110, is unburnt and does not differ greatly from the rest of the assemblage.

There is only one retouched piece not made of flint: SF106, a flake of mudstone, already reasoned (see above $\mathrm{p}$ 217) to have originally formed part of a polished stone axe. The dorsal surface has been ground into three clear longitudinal facets by groups of parallel striations on average $8 \mathrm{~mm}$ long. These are plainly visible to the naked eye. There is no other evidence of polishing within the assemblage and as the axe may well have been introduced as a finished tool it cannot be proved that polishing formed a part of the repertoire of the knappers at Strathallan. The flake has small, irregular, retouched scars along parts of both left and right edges indicating that the usefulness of the stone, together with the fact that it could be worked like flint, were both recognized.

\section{The morphology of the retouched pieces}

Amongst the retouched collection there are several fragments, for example SF100-104 and 109, of larger tools where the surviving morphology has been so affected by breakage that the original shape can no longer be reconstructed. Most of these retain only a short length whether unifacial or bifacial and although it would seem likely that the breakage curtailed the working life of the flake this cannot be certain. One piece, 104, is more interesting as the retouch extends completely around it with the exception of the broken distal end. The irregular, curved shape of the surviving fragment was therefore intentional but it is impossible to suggest the original finished shape.

The rest of the retouched flint pieces are all complete and may be more usefully examined. SF72 is of a very irregular shape and demonstrates the importance of spontaneous function in the use of retouch to create an edge for a particular task. It has only a small, convex stretch of retouch placed upon the thickest part of the flake and does not conform to any recognizable morphological type. SF105 is a particularly small piece on which tiny, irregular retouch has been used to emphasize and strengthen a point at the junction of the distal end and the right side. Such a small piece must have been hafted in order for any force to have been employed in its use and it might have traditionally been called a 'borer' or 'piercer'. SF73, 99 and 110 are all pieces that would traditionally be called scrapers, a term used widely today, and in this paper, to describe a variety of steep, often convex, retouched edge. Two of these pieces, SF110 and 99, are small and chunky with compact steeply retouched edges. SF110 also has evidence of ventral thinning to remove the bulb. SF73 is a larger, thinner flake with retouch around most of its circumference.

SF53 has a triangular shape with a slightly convex base at the distal end of the flake. It is bifacially retouched along the right side and part of the distal while on the straight left side the original, sharp flake edge has been retained. Traditionally such pieces have been called 'petit trachet derivative arrowheads' of Clark's class I (Clark 1934, 35 \& 54-5), although many other names have also been assigned to them, for 
example, 'lopsided arrowheads' (Stevenson, R B K 1947, 180), 'transverse arrowheads' (Wainwright \& Longworth 1971, 257-9), or 'oblique arrowheads' (Green 1980, 38 \& 100); this particular point would be of the Irish type as the flake edge is shorter than the retouched edge). While the suitability of such a piece as an arrowhead cannot be defined it would also lend itself to other tasks.

Four of the pieces, SF54 and 96-98, demonstrate the use of retouch to create a short, steep, convex edge upon large flakes in a recurring position at the left side of the distal end. Three of the flakes are secondary but it is most probable that this reflects the need for size rather than for the presence of cortex. Each piece also has an unretouched flake edge on the right side, on three this has macroscopic edge damage which may have been caused by use. A general similarity of shape demonstrates the importance of that shape in their manufacture but one flake, SF54, where flaws and mishits by the knapper have produced a sharp irregularity on the right side, indicates that this importance was not over-riding. The retouch used to create each edge is not identical, SF96 for example, shows large, broad scars, while SF54 has long, thin ones.

The final two retouched flint pieces were found with burial B. SF107 is a large flake that has been bifacially thinned and formed into a leaf shape. It might traditionally be called a 'knife' or 'spear point'. The presence of ventral retouch means that it cannot be classified as plano-convex. The second piece, SF108, although much smaller, is of a similar shape and might also be called a 'knife'. In this case the retouch is upon the dorsal face only and, as less thinning has taken place, a more sharply convex profile is retained.

One retouched piece remains undescribed, SF106, the mudstone flake. Here the morphology of the original flake has been retained. Small, irregular lengths of retouch on either side have served to create and consolidate the necessary edge, as with SF72 the importance of spontaneous function is demonstrated.

\section{The function of the assemblage}

With such a high percentage of retouched pieces and regular but unretouched flakes compared to the absence of débitage and other evidence of knapping it seems likely that the assemblage represents a collection of working tools brought on to the site ready-made and used, altered and discarded as necessary. In some cases retouching improves a tool's life and efficiency but unretouched flakes may also be used to carry out certain tasks. It is possible to recover detailed functional information from stone tools but this involves complex specialist analysis and has not formed part of the present study so that the actual tasks in question cannot be ascertained.

Two points should be mentioned, however; firstly the number of small fragments of broken retouched pieces amongst the collection and secondly the presence of macroscopic edge damage upon some flakes, both retouched and unretouched. Like breakage, which also exists on both types of flake, edge damage may be caused by many things one of which is use, although it would require microscopic examination, which is outside the scope of the present study, for this to be confirmed.

\section{Cultural and chronological associations of the assemblage}

The limitations of the use of stone artefact evidence have been discussed. However, a few of the flints within this collection do provide morphological parallels elsewhere. A large number of the retouched pieces are broken and impossible to reconstruct. Others, the scrapers, provide only general parallels as they are common on most Neolithic and Bronze Age sites although they have been held to be of significance in association with Grooved ware pottery (Wainwright \& Longworth 1971, 256-7; Manby 1974, 82-3). The single edge retouched point, SF53, has a less widespread occurrence but is morphologically paralleled elsewhere throughout the British Isles and is commonly associated by archaeologists with Grooved ware (Green 1980,109-10). The two other pieces that would traditionally be used as indicators are 107 and 108. As 'knives' they also have been associated with Grooved ware (Wainwright \& Longworth 1971, 256 \& 259-60) although other associations do exist (Evans 1897, 331-7). These latter two are perhaps of more interest as having been purposefully burnt and deposited with the burial. Few burials are definitely associated with Grooved ware but the practice of incorporating flints, worked or not, with burials is widespread and crosses many cultural boundaries. The contextual separation of these two pieces from the rest of the assemblage must be noted in any examination for cultural parallels.

Many factors affect the form of an assemblage and in order to demonstrate similarity between two or more assemblages it is necessary to examine the complete collections, débitage and waste as well as retouched tools, at all levels; material, technology, morphology, function etc as well as culture. Only then can the relative importance of each constraint be evaluated. 


\section{The distribution of pieces within the site}

As most of the collection was located randomly throughout the barrow and there are no indications of knapping floors, nor of other specialized functional deposits, it would seem most likely that the assemblage accumulated through the loss and discarding of working tools prior to and during the erection of the barrow. The small amount of débitage may be accounted for by the resharpening, alteration and breakage of tools whilst in use, in addition to general post-deposition abrasion.

One group of retouched pieces alone stands within a context different from the rest of the collection; SF107-110 were found within cists. Three of these, SF107-109, are heavily burnt. These burnt pieces are not types found elsewhere within the barrow. One, SF109, has been very damaged by the burning but neither of the others is paralleled either morphologically or technologically. This does not mean that they were made by different knappers. SF110, the distal end scraper, is, however, paralleled both morphologically and technologically within the rest of the assemblage and, coming from a inhumation, is not burnt.

\section{SUMMARY}

The assemblage consists of 62 pieces, mainly of flint although pitchstone and quartz were also used in small quantities and mudstone was introduced as a polished axe and re-used after breakage. The flint and quartz were probably gathered locally from a gravel source where it was possible to collect pebbles of flint that were of a large size and of good quality with few flaws. The nodules were reduced using a variety of hammers, with predominantly direct percussion upon artificial, occasionally facetted platforms. From the resultant assemblage of regularly shaped flakes some were selected, upon a basis of quality and size, for retouching. This was done both to shape tools and to create particular working edges. Different methods were used to achieve different types of retouch, for example, percussion with a small stone hammer, pressure with an antler tine or light percussion with an antler or wooden baton. A number of different tool types were made in these ways; some are duplicated within the assemblage. Many survive only as broken fragments where it is impossible to reconstruct the original tool. Some of these pieces may be morphologically paralleled on other sites but it is not yet possible to draw valid cultural information about such parallels. Only the four pieces within the cists were not found under and in the matrix of the mound. It is likely that the assemblage represents a working accumulation of tools and associated waste although the lack of detailed functional analysis means that the exact nature of the tasks concerned cannot be ascertained in the present study. A similar difficulty exists when interpreting the actual behaviour behind the incorporation of the four pieces into the burial.

\section{OTHER ARTEFACTS FROM THE BARROW}

\section{G J Barclay}

SF112 (fig 60) An irregularly shaped stone approximately 110 by $105 \mathrm{~mm}$ and $43 \mathrm{~mm}$ thick. Both faces bear a pecked mark c $10 \mathrm{~mm}$ deep and c $50 \mathrm{~mm}$ in diameter, resembling cupmarks. The edge of the stone has five faces of which four show signs of a little smoothing. The artefact can be interpreted as a portable cupmarked stone. It has however been used as a rubbing tool. The cupmarks do not have the appearance of an abandoned attempt at perforation.

SF113 (pl 16) A jet necklace accompanying burial G, with a food vessel (SF42). There were 136 perforated jet discs (and fragments of about four further discs) varying in diameter from 4 to $8 \mathrm{~mm}$ and between 1 and $2 \mathrm{~mm}$ thick; the broadest were not the thickest. As found the necklace was in four segments of contiguous discs, with gaps between presumably caused by animal burrowing. Seventyone beads were in the undisturbed groups, the remainder scattered in the immediate area. In the first group ( $a-b$ as excavated) the 12 beads were of constant diameter, at the larger end of the scale; likewise the 11 beads of the second group (c-f). In the third group of 17 beads $(g-h)$, the diameter reduced from $g$ towards $h$. In the fourth group $(j-k)$, after the largest break in the necklace, the 37 beads reduced in diameter for the first third of the run and then remained at a constant size. The loose beads can be separated into two groups; 11 over $7 \mathrm{~mm}$ in diameter and 45 of $6 \mathrm{~mm}$ or less. It is suggested that these beads represent the back of the necklace filling the gap between $k$ and $a$.

SF114 (fig 60) A circular disc of siltstone $36 \mathrm{~mm}$ in diameter and $10 \mathrm{~mm}$ thick with an almost perfectly 

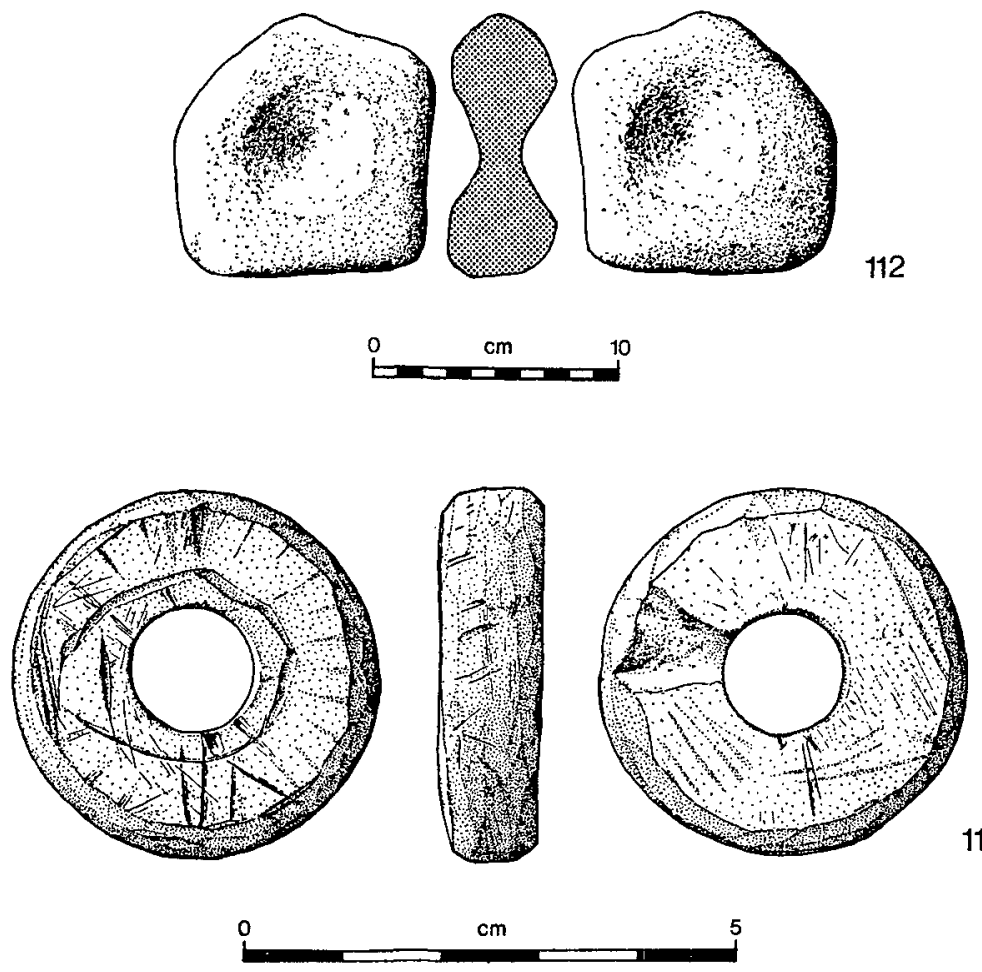

114

FIG 60 Barrow worked stone

circular central perforation $12 \mathrm{~mm}$ in diameter. One surface was very smooth and was decorated with a roughly scored ring round the perforation and a series of radial scored lines, now slightly worn. At places on the outer edge of the surface are deep scratches, parts of a circle removed in places by the working of the edge; perhaps the marks represent the original marking out of the completed object on the smoothed surface of the piece from which it was cut. The perforation was very smooth; only slight scratches marked the surface. The edge of the disc was facetted in such a way as to suggest that the stone had been pared into shape. It was also marked by sharp cuts, possibly the result of a process of manufacture or of the disc's use. The stone is sufficiently soft to have been pared by a flint tool. The reverse surface is not so smooth as the obverse. It was defaced by a broad deep groove, which may have been formed during the manufacture or use of the disc. It may, however, be a flaw in the original stone. Broad shallow smoothed grooves on the reverse may have been caused by the shaping of the disc. There are also sharper narrower cuts.

\section{UNBURNT BONE FROM THE BARROW AT NORTH MAINS}

\section{A Young}

\section{Burial E}

There are only the remains of a calvarium (found inverted). The bone fragments seem very thin this may be due partly to weathering decay of the inner table but it is more likely that it is due rather to the age of the individual. No sutures were recognizable but the skull was probably of a child about 4-6 years old, possibly even younger. 


\title{
CREMATIONS FROM THE BARROW AT NORTH MAINS
}

\author{
C B Denston, Department of Physical Anthropology, University of Cambridge
}

\section{CATALOGUE}

\section{Burial A}

Maximum length of fragments: $101 \mathrm{~mm}$.

Number of individuals: possibly four or five.

Sex and age at death: either (i) three male adults; one female adult, or (ii) two male adults; one female adult; child of about 4-5 years.

The fragments of three adult occipital bones suggested more affinities with male crania than female. However, a large part of a frontal bone displaying the superior margin of the orbits and superciliary ridges, had more affinities with a female than a male cranium. The thickness of other fragments suggested the presence of male and female cranial remains, the closure of a suture in the region of the foramen of a fragment of a parietal bone of a female indicated an age of over 30 years. The frontal bone could not be associated satisfactorily with one of the occipital bones. The presence of two right and a left petrous of temporal bones of differing appearance, suggested three crania. None of the long bone or other postcranial fragments suggested any male individuals. Head and neck portions of femora suggested female, with fused epiphyses indicating adulthood.

Though in general the evidence suggested female individuals, the evidence of the occipital bone fragments cannot be overlooked. Therefore the remains possibly represented three males and at least one female. One occipital could conceivably have been associated with the frontal bone making two males and a female. A fourth portion of occipital bone, and other cranial fragments, one fragment displaying a suture, suggested the presence of child, possibly about 4-5 years old.

\section{Burial B}

Maximum length of fragments: $127 \mathrm{~mm}$.

Number of individuals: possibly four.

Sex and age at death: male adult; female adult; immature; infant/neonate.

The cranial evidence suggested that four individuals were represented. The fragments identified as of the vault of crania varied in thickness, the variation too great to belong to a single cranium. Some were very thin, one fragment suggesting a serrated edge was in the early part of the neonatal period. Duplicated sutures from the same areas of the cranium suggested the presence of two individuals, one possibly male, the other female or immature. A right and a left petrous of temporal bones of varying robustness suggested two individuals in addition to the infant. Robustness of a fragment of occipital bone and a mastoid process suggested an adult male. In favour of a female were the parietal-bregma region of the cranium, the glenoid fossa area and a frontal process of a zygomatic bone. Difference in size of two frontal processes of maxilla suggested male and female, and difference in size of two frontal processes of zygomatic bones suggested a female and an immature crania. Supporting the representation of an immature individual was the presence of a small coronoid process of a mandible and an occipital condyle. Roots of teeth all appeared to be from adult dentitions, and unfused sutures of the cranial vaults suggested neither of the adult individuals were very old.

These conclusions were substantiated by the post-cranial remains, the thickness of the long bone shaft fragments suggesting the presence of more than one individual. Fragments of the shaft and head area of humerus suggested male affinities while other fragments of the humeral head and those of the shaft of other long bones suggested a less robust individual, possibly a female. Part of a pubic symphysis of an innominate bone was present; the symphyseal surface displayed a ridge and furrow appearance suggesting an age of 20-24 years. The size of the symphysis suggests a female rather than a male. Another portion of the pelvis was preserved, as part of an acetabulum, too small to be of an adult male. It was representative either of an adult female, or an immature individual. The fusion of the ilium, ischium and pubic bones were complete in the acetabulum, and if classed with the immature remains would place them at puberty. The acromion process of a scapula, distal articular extremity of a femur, fragments of clavicle and olecranon process of an ulna may also have been portions of immature bones.

Intact phalanges suggested they were of an adult; from their size a female. The proximal articular 
extremity of a tibia suggested an adult female, and a portion of an iliac crest of an innominate bone, an adult male.

\section{Burial C}

Maximum length of fragments: $127 \mathrm{~mm}$.

Number of individuals: possibly at least eight.

Sex and age at death: male and female adults; adolescence/puberty; late childhood; early childhood; foetal/birth.

The thickness of the cranial fragments suggested a range of ages from extreme youth (foetal-birth) to adult. Some fragments were very thin, one presenting a sutural surface suggesting an age of foetal to birth. Other fragments with sutural edges suggested ages of 6 months to a year, approximately 6 years, and 6-12 years. There was no suture evidence to suggest individuals over 30 years of age. The development of the superior margin of a fragment of an orbit of a frontal bone should belong to a cranium of a child of 2-3 years. Eleven petrous portions of temporal bones, five from the right side of the cranium, and six from the left, suggested the presence of at least six individuals. Variation in the size of these bones and in the dimensions of the apertures of their foramena suggested that three were childrens'. The mastoid area of three fragments, all from the right side of the cranium, and two mastoid fragments from the left side displayed more male than female affinities. The auditory meatus-mastoid area as displayed on another fragment, lacking the robustness of the others, was possibly female. The internal and external protruberance of fragments of three occipital bones suggested two males and a female. Four left mandibular condyles possibly suggested two adult males, an immature individual perhaps at puberty, and a younger individual approximately 6 years old. The basisphenoid-basioccipital area of the cranium was represented by two fragments, one possibly male, the other female which suggested ages in excess of 23 years. Most tooth roots were fully formed, but the crown development of two premolars would suggest a child approximately 6 years old. These two crowns survived the combustion because they were enclosed in the alveolus and unerupted.

TABLE 8

Weight in grammes of the Barrow cremation burials

\begin{tabular}{|c|c|c|c|c|c|c|c|}
\hline & A & $\mathbf{B}$ & C & $\mathbf{D}$ & $\mathbf{F}$ & $\mathbf{H}$ & $\mathbf{J}$ \\
\hline 1. Cranium & $\begin{array}{r}304 \cdot 3 \\
6 \cdot 3\end{array}$ & $\begin{array}{r}329 \cdot 9 \\
10 \cdot 5\end{array}$ & $\begin{array}{r}1096 \cdot 0 \\
89 \cdot 0\end{array}$ & $\begin{array}{r}582.0 \\
18.8\end{array}$ & $87 \cdot 6$ & $223 \cdot 8$ & $53 \cdot 4$ \\
\hline 3. Teeth & $4 \cdot 3$ & 3.0 & $\begin{array}{l}89 \cdot 0 \\
32 \cdot 2\end{array}$ & $\begin{array}{l}18 \cdot 8 \\
15 \cdot 7\end{array}$ & $\begin{array}{l}2.0 \\
0.7\end{array}$ & $\begin{array}{l}18 \cdot 2 \\
10 \cdot 0\end{array}$ & \\
\hline 4. Femur & $54 \cdot 0$ & $124 \cdot 3$ & $358 \cdot 8$ & & $64 \cdot 6$ & 130.0 & $42 \cdot 1$ \\
\hline 5. Tibia & $48 \cdot 0$ & $130 \cdot 8$ & $284 \cdot 0$ & & $46 \cdot 4$ & $89 \cdot 0$ & $21 \cdot 0$ \\
\hline 6. Fibula & & $21 \cdot 5$ & $95 \cdot 0$ & & 1.9 & $43 \cdot 5$ & \\
\hline 7. Radius & $17 \cdot 8$ & $48 \cdot 4$ & $91 \cdot 3$ & & $10 \cdot 0$ & $41 \cdot 1$ & \\
\hline 8. Ulna & & $34 \cdot 7$ & $82 \cdot 5$ & & $2 \cdot 1$ & $35 \cdot 5$ & \\
\hline 9. Humerus & $34 \cdot 3$ & $72 \cdot 6$ & $311 \cdot 5$ & & $41 \cdot 6$ & $116 \cdot 1$ & $13 \cdot 0$ \\
\hline 10. Pelvis & $44 \cdot 0$ & $39 \cdot 8$ & $145 \cdot 2$ & $49 \cdot 0$ & $23 \cdot 4$ & $93 \cdot 0$ & $1 \cdot 2$ \\
\hline 11. Vertebrae & $70 \cdot 0$ & $29 \cdot 2$ & $296 \cdot 5$ & $56 \cdot 0$ & $40 \cdot 0$ & $78 \cdot 4$ & $2 \cdot 5$ \\
\hline 12. Clavicle & $1 \cdot 8$ & $9 \cdot 0$ & $7 \cdot 5$ & $3 \cdot 3$ & & $11 \cdot 9$ & \\
\hline 13. Rib & $44 \cdot 6$ & $20 \cdot 0$ & $334 \cdot 6$ & $89 \cdot 8$ & $9 \cdot 8$ & $20 \cdot 6$ & $2 \cdot 5$ \\
\hline 14. Metacarpal \& & & & & & & & \\
\hline $\begin{array}{l}\text { Metatarsal } \\
\text { Phalanges }\end{array}$ & $43 \cdot 6$ & $28 \cdot 7$ & $267 \cdot 0$ & $50 \cdot 4$ & $18 \cdot 0$ & $44 \cdot 0$ & $2 \cdot 0$ \\
\hline 15. Patella & $1 \cdot 7$ & $4 \cdot 3$ & $13 \cdot 0$ & $4 \cdot 0$ & $5 \cdot 6$ & $4 \cdot 5$ & \\
\hline 16. Scapula & $2 \cdot 1$ & $5 \cdot 8$ & $82 \cdot 0$ & $2 \cdot 7$ & & $16 \cdot 4$ & \\
\hline 17. Calcaneum & $2 \cdot 3$ & $2 \cdot 3$ & & & & & \\
\hline 18. Talus & $4 \cdot 0$ & $12 \cdot 0$ & & & & & \\
\hline $\begin{array}{l}\text { 19. Carpal \& } \\
\text { tarsal bones }\end{array}$ & $10 \cdot 4$ & $10 \cdot 0$ & $51 \cdot 5$ & $11 \cdot 0$ & $\begin{array}{l}\text { see } 14 \\
\text { above }\end{array}$ & $18 \cdot 2$ & \\
\hline 20. Misc long bones & $366 \cdot 2$ & $329 \cdot 0$ & $1849 \cdot 5$ & $803 \cdot 0$ & $221 \cdot 0$ & $315 \cdot 5$ & $242 \cdot 5$ \\
\hline $\begin{array}{l}\text { 21. Misc bone } \\
\text { 22. Sternum }\end{array}$ & $524 \cdot 4$ & $632 \cdot 1$ & $1273 \cdot 0$ & $1305 \cdot 2$ & $\begin{array}{r}305 \cdot 3 \\
1 \cdot 5\end{array}$ & $587 \cdot 3$ & $205 \cdot 0$ \\
\hline Total & $1585 \cdot 1$ & $1897 \cdot 9$ & $6760 \cdot 1$ & 2990.9 & $881 \cdot 5$ & $1897 \cdot 0$ & $585 \cdot 2$ \\
\hline
\end{tabular}


The development displayed in fragments of the shaft of femur, humerus, radius and ulna suggested at least one rather robust male. These bones need not necessarily have been associated and could have represented more than one male individual. Other shaft and extremity fragments of long bones, suggested the presence of male, female, and immature individuals. Six proximal articular extremities of radius, by size, could have represented three adult males substantiating the evidence of the mastoid area of the cranium.

Postcranial bones other than long bones:

i male: glenoid fossa of the scapula, iliac crest of innominate bone, odontoid process of the axis-two, body portions of vertebrae, articular facets of atlas, scaphoid bones of the wrist, ribs, and metacarpal and metatarsal bones.

ii female: clavicle, iliac crest of innominate bone, acetabulum of innominate bone, odontoid process of the axis, articular facets of the atlas, scaphoid bone of the wrist, vertebrae, ribs and metacarpal and metatarsal bones.

iii immature: (?) clavicle, odontoid process, (?) articular facets of atlas, ribs, distal phalanges of the hand.

Twenty-two intact distal phalanges of the hand, displaying consolidation of the epiphyses, indicated that at least three individuals, other than children, were represented. The two portions from the iliac crest, one possibly from a female pelvis, the other male, both displaying consolidation of the epiphyses, suggested an individual at least 25 years old. Also, on every fragment where the distal extremity of the metacarpal and metatarsal bones were preserved the epiphyses were consolidated to the shaft.

\section{Burial D}

Maximum length of fragments: $67 \mathrm{~mm}$.

Number of individuals: at least seven.

Sex and age at death: (?) foetal up to adult; (?) possibly a male adult; one female adult.

Probable animal bones found in this deposit were submitted to Barnetson (p 231).

Tentative ages at death:

One adult male: under 30 years.

One adult female: under 30 years.

*Foetal-birth: superior margin, orbit; suture.

*6 months.

1 year: basilar portion, occipital bone.

*1 year - 18 months: neck-head, femur.

*18 months -2 years.

2 years: neck-head, femur; basilar portion, occipital bone; distal phalanges, hand.

*2-3 years: internal protuberance, occipital bone.

4-5 years: head of a femur.

5-6 years: head of a femur.

*6 years: mandibular condyle.

6-7 years: head of a femur.

9-10 years: distal phalange of the thumb.

*11-12 years.

Adolescent: acetabulum, innominate bone (or female); epiphysis, metatarsal bone, unfused.

$\left(^{*}=\right.$ Age assessed from the formation of, or eruption of teeth of complete skulls, not associated with this excavation.)

The varying thickness, and fragments of crania displaying sutures, overwhelmingly suggested the presence of more immature individuals than adults. None of the suture patterns suggested advanced age: most were unfused, and some cranial fragments were very thin, possibly representing one or more foetal or neonatal individuals. The adults represented by the cranial fragments seem to have been under 30 years of age. Endocranially, the sutures were not fused; the thickness and pattern of the sutures suggested ages from foetal-birth, through early childhood and adolescence to adult. Eleven petrous portions of 
temporal bones, seven of which came from the left side of the cranium, were evidence that at least seven individuals were present. The size of at least four of these bones suggested that they came from infant crania, the rest possibly covered a period from late childhood to adult. The only mastoid process present, gauged by size and robustness, had more affinities with a male than a female cranium. Right and left mandibular condyles suggested the presence of at least two adults. A fragment of an occipital bone displaying the internal protuberance was more inclined to a female than to a male cranium; the pattern of a suture suggested an adult.

The fragments of long bones suggested various ages, none robust; the only possibly male fragment was that of a femur. In favour of female were the distal extremities of a fibula and ulna displaying consolidation of the epiphyses. The consolidation of epiphyses to shaft was also noted on the metatarsals and the distal phalanges of the hand. On a fragment of vertebra the epiphyseal was also consolidated to the body portion.

\section{Burial $F$}

Maximum length of fragments: $75 \mathrm{~mm}$.

Number of individuals: one.

Sex and age at death: female; young adult.

There was no evidence for more than one individual. The general lack of robustness of cranial and postcranial fragments suggested a female. Epiphyseal union was complete, as illustrated at the femoral head and on an intact phalange. The saggital suture, and that displayed at the bregma were unfused, suggesting the individual was relatively young.

\section{Burial $H$}

Maximum length of fragments: $12 \mathrm{~mm}$.

Number of individuals: at least two.

Sex and age at death: male adult; female adult.

The remains did not appear to be of a single individual. Three coronoid processes of the mandible, two of which were duplicated from the right side, and the protuberance and crest of the endocranial surface of two separate fragments of occipital bone of the cranium, suggested the presence of at least two individuals. The difference in size, or robustness, of features of these and other fragments suggested that one individual was a male and the other a female. Intact phalanges, and fragments from the extremities of various other bones of the skeleton displayed completed epiphyseal union indicating that both individuals were adult. The smoothness and inactivity of the symphyseal face of an incomplete pubic symphysis was consistent with an age of 39-44 years (? male). A fragment, possibly from the fourth segment of the sacrum suggested there was not complete fusion at the inferior surface, implying an age of 20-25 years. The fragment had two lateral surfaces, plus the inferior surface, and was possibly representative of the female individual. It is suggested that a large mastoid process, and a well developed superciliary ridge of frontal bone of the cranium, were representative of a female individual.

\section{Burial J}

Maximum length of fragments: $56 \mathrm{~mm}$.

Number of individuals: one.

Sex and age at death: (?) female adult.

The examination of the remains revealed no duplicated portions of bones, suggesting that only one individual was represented, possibly a female. A small part of the iliac crest of an innominate bone displayed consolidation of the epiphysis.

\section{Burial $K$}

Fragments of long bone and cranium found scattered through part of the stone core. Most of the scattered, but associable, fragments had the appearance of being human, though small in size. The lack of robustness of the fragments suggested female affinities rather than male. Probably an adult. 


\section{IDENTIFIABLE BURNT ANIMAL BONE FROM THE BARROW AT NORTH MAINS}

\section{P D Barnetson, Department of Archaeology, University of Edinburgh}

Identifiable fragments were found only in Burial C.

Pig

Right humerus fragment, proximal epiphysis unfused

Right humerus proximal epiphysis fragment

Right radius: fragment of proximal end, epiphysis fused

fragment of diaphysis

distal end, epiphysis unfused

Right ulna: proximal end, epiphysis fused

fragment of proximal end

distal end, unfused

styloid process, unfused

Almost complete radial carpal and intermediate carpal

2nd phalanx (abaxial digit)

Pig (?) Fragment of scapula, glenoid cavity

Fragment of scapula

Fragment of distal humerus (?)

Bos (?) Rib fragment

\section{DISCUSSION}

\section{G J Barclay}

PERIODS I AND II

The features sealed under the old land surface (Period $I$ ) had been isolated from the activity surface from which they were dug by the disturbance caused by the subsequent agricultural use of the land (Period II). As has been described above ( $p$ 191) two of these features have been interpreted as postholes, though constructed in different ways. Their presence and that of the artefacts found in the fossil topsoil might suggest that a settlement lay relatively close to the site, though many of the finds may have originated in midden material dumped in the area in either period. Whether the postholes were part of the settlement itself or associated with a peripheral activity could not be decided by excavation. Period II, certainly a time of agricultural use of the land under the mound, may have been an extension of the Period I use of the land, or might reflect a change in the location of the settlement, or even a much later use of the land, after a break in the continuity of occupation.

The only feature which could be assigned to Period II was the corrugation of the old land surface. In the $\mathbf{N}$ half of the site it was well defined but in the area which was to become the central enclosure of the Period III monument no trace was found. In the S only faint indications, too insubstantial to plan, were noted, but in both the SE and SW quadrants there were single broad, shallow, steep-sided slots. These features may have been formed by the removal of turf with a spade and could not be equated with the smooth contours of the ridges and furrows to the $\mathrm{N}$. Although possibly the result of an agricultural process it is perhaps more likely that they were formed during the earlier part of Period III.

The ridging at North Mains was probably formed using a spade or hoe to pile up the soil. The function of the ridges is not clear - they may have served to provide better drainage or greater soil depth for cultivation. No spade-marks or ard-marks were found but it is likely that the 
pattern of oriented clay deposition in the upper $4-5 \mathrm{~cm}$ represents shallow cultivation, by hoe or spade.

Bradley (1978) has noted that furrows existed under the Bronze Age barrows at Ascot, Berkshire (Bradley \& Keith-Lucas 1975), Latch Farm, Hampshire (Piggott, C 1938), Simondstown, Glamorgan (Fox 1937; 1959) and Wind Hill, Lancashire (Tyson 1972). Ridging was also found under the rather earlier long barrow at Fussell's Lodge, Wiltshire through insufficient information on the features was given in the published report (Ashbee 1966). In Scotland, ridging of the early second millennium survived under peat at Machrie, Isle of Arran (Barber, forthcoming). Ashmore, in an interim statement on the first season of excavation at Callanish, Isle of Lewis, has described slight surviving evidence for ridging on the pre-peat surface.

Romans and Robertson (infra \& 1983) have suggested that the puddled gleyed surface of the fossil topsoil was formed by stock, perhaps indicating a change of use from cultivation to pasture. The rather greater erosion of ridges under the $\mathrm{S}$ half of the mound might indicate that they were formed longer before the erection of the mound than those in the $\mathrm{N}$ half.

Little environmental evidence was available from the fossil topsoil. Hulme and Shirriffs have noted, in their report on $\mathrm{p} 270$, that the surviving pollen in the soil was too scanty to allow firm conclusions to be drawn, but the available evidence suggests an open landscape with light woodland, and signs of human disturbance.

PERIOD III

The structures under the mound apparently consisted of six separate main elements: (1) the two posts within the enclosure (F12 and F13); (2) the grooves radiating to $\mathrm{S}, \mathrm{E}$ and $\mathrm{W} ;(3$ and 4) the S and N parts of the central enclosure; (5) the radial fences and (6) the arrangement of stakes and grooves at the NE of the enclosure. The sequence of the construction of these features could not be completely established by excavation; however, some relationships were clear. The grooves (2) radiated from F12 and respected F13 and therefore probably post-dated them. The grooves were cut by posts of element 3 and the radial fences, including those set out along the grooves, halted at the edge of the enclosure. While no stratigraphic relationship could be found between the $\mathrm{S}$ and $\mathrm{N}$ halves of the enclosure it is suggested that the more massively built ( $\mathrm{S}$ ) preceded the other and that the former had a separate function prior to the completion of the circle by the latter.

The erection of the radial fences probably began before the dumping of the material of the ring-bank; however, the root disturbance near the edge of the mound might have disguised the rising of the stake-fences on to mound material ringing the site edge. It is suggested that some of the shorter fences visible on the old land surface rose on to the ring-bank when it had encroached further on the enclosure, and were therefore built after dumping had begun.

The suggested sequence of events in Period III, phase A is therefore:

i F12 and F13; posts erected

ii digging of grooves F14, F15, F16, F17

iii erection of $\mathrm{S}$ half of enclosure and possible separate use of this structure; (?) burial at this stage

iv erection of the $\mathrm{N}$ half of the enclosure, including the building of the entrance; possibly contemporary with $\mathbf{v}$

$v$ (a) the erection of the greater part of the radial fence system before and during the erection of the ring-bank

(b) the use of the enclosure for (?) burial (or activity secondary to a burial at iii above) before during or after the erection of the ring-bank 
The chronological relationship between $\mathrm{v} a$ and $\mathrm{b}$ is not certain. All the activities described could have been broadly contemporary. This sequence was followed by the construction of the ring-bank and the covering of the deposits in the centre by the gravel and silt embanking of the inner face of the timber enclosure (Period III, phase B).

The elements of the substructure were represented only by negative features; postholes, stakeholes and grooves. In the $S$ half of the enclosure some impressions of posts were found in mound material. Fragmentary impressions of one radial fence were preserved in the Period III, phase $\mathrm{C}$ mound which may show how the lower radial fences were made. Of the remaining structural features little can be said with certainty. The two posts (F12 and 13) set up near the centre (structural element 1 (see p 193)) were of unknown height and function. No trace of them was found in the embanking material, which might have been expected to preserve impressions; however this is not proof that they were not still standing when the mound was built.

As the grooves radiated from F12 it is possible that the post was set up to mark the central spot of the projected monument and that the grooves served a similar planning function. It is also possible that the later enclosure was lightly roofed and that this post supported its rafters. The $\mathrm{S}$ portion of the enclosure, slightly more than half of the circle, formed, with the grooves, a $\mathrm{N}$ facing complex of features; there was no groove to the $\mathrm{N}$ of F12. It is possible that this arc served a separate function in the ritual of the presumed burial.

Later in Period III, material of different types was dumped inside and outside the enclosure with no apparent mixing. It must therefore be presumed that some form of fencing linked the uprights. In both $\mathrm{N}$ and $\mathrm{S}$ halves the uprights were arranged in segments of straight lines, suggesting that they were placed to support sections of fencing.

The short grooves and stakeholes at the NE may have marked the entrance to the completed enclosure but no trace of the structure was preserved in the body of the mound. However, the coincidence of this feature with the possible access bay through the ring-bank and the disturbed path on the old land surface from the NE to the centre suggests that it may have been the first of a sequence of entrances to the central enclosure, in use until late in the building of the mound.

The radial fences, which formed the framework of the mound, were represented in the lower half only by the arrangement of stakeholes on the old land surface. That they were fences was proved by the distinct separation of materials in adjoining bays. The pattern of discontinuities within the lines of stakes, as $\mathrm{p} 237$, suggested the use of sections of fencing. The four short lines of stakeholes noted on the old land surface (fence nos 3, 7, 9 and 12) may be interpreted as fences erected after the building of the ring-bank had begun; the few metres of fence near the centre would have been set on the OLS, the remainder on the ring-bank, leaving no trace on the OLS. Fence no 9 was represented only by one stakehole and a line of 'dimples' on the OLS, though it was clearly in existence during the erection of the ring-bank. It is suggested that the fence was built when the ring-bank had already encroached on the central area and that the dimples showed where fence stakes had penetrated the dump and slightly disturbed the old land surface.

Other features described in the earlier part of the report remain to be discussed. Within the enclosure the areas of old land surface at the centre and to the NE which had a different appearance from the remainder of the OLS may have represented the concentration of activity in those areas (as mentioned above). Pare's phosphate survey (infra p 269) has confirmed that organic material was placed at the centre and in other parts of the enclosure. It is suggested that after the completion of the enclosure one or more bodies were placed near the centre, resulting in the traffic between there and the entrance at the NE. The burials would have been covered at the beginning of Period III, phase B. It is possible that the erection of the ring-bank had proceeded some way before the burials were deposited and that they were placed immediately prior to the 
dumping of the embankment material in the enclosure. No grave goods had survived. Though the high surface phosphate concentrations within the structure might suggest the presence of human burials, the deposition of other organic material, accounting for some or all of the high phosphate levels, should not be dismissed as an interpretation.

The enclosure, if considered as a two phase structure, the $\mathrm{S}$ half standing as an independent feature for an indeterminate time, may have had a separate function prior to the supposed burial(s). The old land surface showed signs of disturbance, perhaps indicating traffic between the differently coloured and textured central area of the enclosure and the presumed entrance feature at the NE. This would indicate use of the enclosure after the $\mathrm{N}$ half was built, as traffic would probably not otherwise be channelled thus. There is no surviving evidence for any use of the semi-circular $\mathrm{S}$ half but given the structural difference between $\mathrm{S}$ and $\mathrm{N}$ portions and the presumption of the difference reflecting a purpose, a deliberately planned ceremonial event or series of events associated with the mound and the possible burials can be suggested with some confidence. Whether the presumed burials were deposited before or after the completion of the enclosure is not clear. The traffic which, it is suggested, is reflected on the old land surface could be associated with a post-deposition activity.

The ring-bank rose between 0.4 and $0.8 \mathrm{~m}$ up the wall of the central enclosure before the dumping of the stone layer on its top (Period III, phase $\mathrm{B} / \mathrm{C}$ ). The nature of the structure of the enclosure is not known in any detail, therefore it cannot be stated with certainty whether or not the fence could bear the weight of spoil unaided. The apparently very flimsy nature of the $\mathrm{N}$ half of the enclosure would suggest that it could not do so but there was no evidence for fence collapse. Thus, while the deposits in the enclosure could have been made during the building of the ringbank, they were probably not made once the ring-bank was completed, as the fence, at least at the $\mathrm{N}$, could not have borne the weight. The first fill of the central structure would probably therefore have been deposited contemporarily with the latter parts of the ring-bank, deliberately dumped to revet the fence and, most likely, to cover the presumed burials.

If the burials were placed in the central enclosure near the end of the building of the ringbank, immediately before the dumping of the enclosure fill and embanking, then the bay between fences 2 and 3, which may have provided access to fill the centre later in the construction process, takes on an added significance. The entrance to the enclosure, as suggested by the evidence of the arrangement of stakes and the disturbance between these and the centre, seems to have lain at the $\mathrm{NE}$, next to this bay (the 'access bay'). It is possible that the bay, apparently illed differently from the rest, provided a path to the centre. This is discussed below ( $p$ 236).

Within the enclosure, and to limited extent outside it on the $S$ side, a series of narrow ridges was noted on the OLS, interpreted as the casts of light poles and twigs left on the surface. They may have been discarded after the building of the central enclosure or the radial fences or, less likely, may be the remnants of a light roof over the enclosure. It is also possible that they were, at least in the central area, part of a deliberate deposit of foliage, either immediately before or after the placing of the burials.

Elsewhere on the old land surface were features worthy of comment. At various points around the central enclosure, and in two cases within it, were stakeholes apparently not associated with the radial fences. To the $\mathrm{W}$ of the enclosure a line of stakes parallel to its boundary, and some $1.5 \mathrm{~m}$ distant from it, was noted. None of the other stakes were grouped so coherently but all may represent parts of additional sub-mound structures, perhaps of limited duration.

The two hurdle impressions, found to the $\mathrm{N}$ and $\mathrm{E}$ of the central enclosure (described on p 195), seem smaller than the fencing units probably used in the construction of the radial fences and therefore may not merely have been discarded surplus pieces. Romans has noted that the old 
land surface would have collected surface water; as the better preserved of the two impressions lay in the hollow between two ridges on the old land surface, it is possible that it was placed there to allow dry-shod access across a wet patch during the use of the sub-mound structures. Low mounds of soil were found piled against the excavated parts of fences 6 and 7, perhaps to support unstable sections of fencing (fig 40). A deposit of charcoal found near the $\mathrm{N}$ edge of the mound at the boundary of the OLS and the turf dump of Period III, phase C (made up of redeposited OLS) was submitted for radiocarbon dating. The assay was 1855 bc \pm 100 (GU-1134).

\section{PERIOD III - THE CONSTRUCTION OF THE MOUND}

The sequence of events from the beginning of the dumping of material to the capping of the mound with stones has been described above ( $p$ 195); little remains to be said. The mound was built within the framework of the central and radial fences until that system was abandoned near the top of the mound, perhaps for practical reasons, because of the reduction of the working area. Some effort was expended in preserving the shape of the central enclosure, firstly by the internal embanking of its boundary fence and subsequently by the extension of its fence upwards and the dumping of stones within it. Within the stone capping, at the top of the mound, was a circular setting of larger boulders. Although its diameter was greater it is possible that it was the last reflection of the circular substructure of the barrow.

Stone was used in some quantity in the mound, concentrated in the fill of the central enclosure, the stone capping, and the lenses of stone separating phases B and C. Additionally, one bay (between fences 9 and 10) in the SE quadrant was filled with stones at a high level (p 198, pl 14). The soil report has pointed to the sub-aerial erosion of much of the stone, suggesting that its original source was in field clearance and that clearance heaps may have been dismantled to provide material for the mound; this would incidentally have replaced some of the land sacrificed to the mound.

At various stages individual and collective decisions were made about the materials to be used. The stone layer appeared consistently at the end of Period III, phase B, even in the bay between fences 2 and 3 (the proposed access bay). In the SE quadrant, where the fine waterdeposited silt was struck in the ditch at a higher level than elsewhere, and where it was used more extensively above the stone layer than in other parts of the mound, it was superseded by turf. The compact silt was usually located (by augering by Romans and Robertson) at or somewhat below the base of the quarry ditch, except in the SE quadrant where the gravel/silt interface was about $1 \mathrm{~m}$ below the ground surface. It is probable that ground water would accumulate above the rather impermeable silt. It would therefore be difficult to continue to dig the silt available in the ditch in the SE once the general water table was reached, and at this point it is probable the builders turned to turf as an easier alternative source of material. Elsewhere on the mound turf was used later only in a relatively thin layer over the whole surface prior to the fire on the top. The result of the deposition of this layer was to even-off the rather irregular surface of the barrow; this may have been the intention of the builders. In the SE quadrant, however, the upper $2 \mathrm{~m}$ of the barrow was built of turf.

Romans and Robertson have identified a break in the dumping of turf in the SE quadrant soon after it began - which may show a short break in the construction of the mound, perhaps even caused by the rain which brought about the clay translocation. The length of the break is difficult to assess: the evidence suggests a few weeks or months, during which rainfall was heavy. There is no evidence for a break of any length between this point and the end of phase E.

The use of the hollow left on top of the mound at the end of Period III, phase C, for the setting of a fire was presumably part of the ritual aspect of the building of the mound. It is clear from 
the soil report that carbon from the fire was washed into the material below before that material had been consolidated, and, therefore, that there had been virtually no break between the dumping of the turf layer covering most of the mound and the setting of the fire. The fire was not a pyre; no burnt bone was found. Burial $\mathrm{C}$ might, because of its depth, have been dug in from this surface but as no charcoal was found in the pit it was clearly not done after the fire. The translocation of clay and carbon from the fire surface (phase D) must have taken place prior to the dumping of the material which filled the hollow (phase E) but the interval cannot be quantified.

The stone capping over the mound must originally have been more uniform than it appeared at the beginning of the excavation. The concentration of natural disturbance on the $\mathrm{E}$ has left the NW quadrant as the best preserved (pl 15). The stone was laid once the turf layer had, perhaps, eroded slightly from the flanks of the mound, but this process could have occurred in a relatively short time. The contemporaneity of the larger stone concentration at the top cannot be proved, and the relationship of the cupmarked slab to this and the rest of the mound must remain a mystery. The irregular lines of boulders visible across the face of the stone capping may be interpreted as attempts to stabilize the layer during its deposition.

Two charcoal samples from the phases of mound construction were radiocarbon dated. The first (III B) was sealed under the stone layer on top of the ring-bank phase of the mound in the NW quadrant (1785bc \pm 85 ; (GU-1103)); the second (III C/D) was from immediately below the burning surface at the top of the mound (1665bc \pm 85 ; (GU-1102)). Taken with the date from the charcoal found at the interface of the OLS and the III C turf layer at the edge of the mound, it is clear that the construction of the mound took place in the first half of the second millennium bc; this conclusion was supported by the presence of the food vessel sherd in the Period III, phase B embanking of the central enclosure.

The bay forméd by fences 2 and 3 in the NE quadrant was apparently filled differently from the others. At the end of the ring-bank stage of construction, when a layer of stones was placed over the surface of the bank, from its crest to the central enclosure, it was laid at a substantially lower level in this one bay. In all but this one bay the level of material of the ring-bank when completed rose from the outer edge of the mound to a crest, and then dropped down towards the centre. In the bay between fences 2 and 3 the dump rose gently from the outer edge towards the enclosure. Later, when the construction materials used changed from quarried spoil to turf in the NE, NW and SW quadrants, the bay had been filled to a much lower level with quarried material than those to $\mathrm{N}$ and $\mathrm{S}$. The fills of the flanking bays were arranged to slope down towards fences 2 and 3 to a greater extent than they did against their other fences.

It is suggested therefore that throughout the construction of the ring-bank and subsequently, until the fence system was abandoned, this bay was always filled to a lower level than the flanking bays and that the arrangement of the fills of the flanking bays was deliberately designed so that the strain on fences 2 and 3 was reduced. This bay coincided with the possible entrance to the central enclosure and the apparently disturbed path between it and the enclosure's centre. It is suggested that the bay was used for access to the central area for the deposition of the presumed burials and subsequently for the filling of the enclosure, first with silt and gravel and then with stone. Whether the presumed burials were deposited before or after the completion of the enclosure is not clear. The traffic which, it is suggested, is reflected in the old land surface, might be associated with a post-deposition activity.

It is structurally possible that a substantial part of the ring-bank had been erected around and against the central enclosure prior to the dumping of the fill of the enclosure. In that case the access bay postulated in the NE quadrant between fences 2 and 3 takes on an added significance. Its alignment with the entrance feature in the enclosure might imply that they were associ- 
ated, and that the bay may have been used to allow access to the centre to fill it. However, it may also have been designed for access to the central area once the ring-bank had been at least partly built, for the purpose of conveying the body, bodies or other organic materials, possibly including grave-goods, to the enclosure, prior to the first filling of the enclosure.

\section{Summary of evidence for the organization of labour and materials}

The radial fences which formed the bays seem to have acted only as labour divisions; they could not have been significant load-bearing elements in the structure. Nor could they have had the strength to allow the filling of one bay without at least a certain amount of material being dumped in the flanking ones. It is likely therefore that most, if not all of the bays were filled at the same time. The 'access' bay between fences 2 and 3, as has been mentioned above ( $p$ 236), was consistently filled to a lower level than those flanking it, which were, however, filled in such a way as to avoid putting excessive pressure against the fences bounding the 'access bay'.

The lateral ridge noted in the $\mathrm{S}$ ditch cutting, and the more substantial causeway at the NNE might suggest that the ditch, like the mound, was made in segments. It may be suggested, in view of the foregoing, that the mound was built by a number of gangs, each responsible for one or more bays, digging the spoil required from the adjacent part of the ditch. The collection of other materials, the turf and stone, may have been organized on a different basis.

The material in the ring-bank, as has been described above ( $p$ 196), was initially dumped beside the inner edge of the ditch and subsequently down the slope of the resultant bank towards the central enclosure. It may therefore be suggested that the labour force for this phase of construction was organized with at least one person per bay quarrying and one standing on the crest of the bank pulling up the spoil; approximately 40 people would be required. It is distinctly possible that larger teams were used, and given the additional work area - the central enclosure a group of up to 100 persons may have been responsible for the earthmoving. It is possible that the same personnel may have undertaken the construction both of the substructure of the mound and the extensions upwards of the various fences during the building, or, alternatively, a further (? specialist) group may have been responsible for the timberwork - gathering materials, constructing hurdles and erecting the framework.

Three types of timber were used: (i) the relatively substantial posts of the $S$ half of the central enclosure, (ii) the sails of the hurdles and the other stakes visible on the Old Land Surface and (iii) the wattles of the hurdles. The ranges of diameters of the pieces, as represented by casts and sockets, are (i) c $20-30 \mathrm{~cm}$, (ii) $1-5 \mathrm{~cm}$ (predominantly $2-3 \mathrm{~cm}$ ) and (iii) $2-7 \mathrm{~cm}$ (predominantly c $4 \mathrm{~cm}$ ).

In excess of $200 \mathrm{~m}$ of fencing was used in the substructure of the mound. As the height of the hurdles is unknown no meaningful estimate can be made for the fencing in the upper levels. There is some evidence that the fences were not built as single units but in several sections, about $2 \mathrm{~m}$ in length, using four of five uprights set both close together and wide apart. The ends of these sections are perhaps marked by slight changes in the directions of the lines of the stakes and by the occurrence of pairs of stakeholes, which could be interpreted as marking the overlap of segments of fencing. Coles (1976) has discussed hurdling preserved in the Somerset Levels.

It has been argued above that the uprights of the central enclosure were linked by hurdling. It is interesting, therefore, to note that these uprights are set in straight segments, both in the $S$ and $\mathrm{N}$ halves, varying in length from $1.3 \mathrm{~m}$ (at the $\mathrm{S}$ ) to $4 \mathrm{~m}$ (at the $\mathrm{NW}$ ). Whereas hurdling could have incorporated the slender poles of the $\mathrm{N}$ half of the enclosure, the stouter posts of the $\mathrm{S}$ could not have formed the uprights of a woven hurdle. It is likelier that prefabricated hurdles were attached to these uprights. 


\section{PARALLELS FOR THE STRUCTURE}

Though the North Mains barrow as a whole cannot, at the moment, be paralleled, certain elements of the structure can. The central enclosure, if taken in its complete state, can be compared with the single stake-circles found under many southern barrows, listed in a recent study of these sites by Kenworthy (1977). Though the evidence from many is of poor quality, the better excavations have shown examples of stakes surviving during the building of the barrow. The central areas in several barrows had been filled, but in the form of a turf stack, rather than the filling of an open space left in the centre of a ring-bank; eg Farncombe Down, Berkshire (Rahtz 1962); Pond Cairn, Glamorgan (Fox 1937).

For the enclosure as it stood after only the $S$ half was erected, there are no precise parallels known to the author. It is conceivable that some stake-circles already known under barrows may also be two phase, but without the use of different sizes of timber to show it clearly. At Chippenham 5, Cambridgeshire (Leaf 1940) a possibly semi-circular post setting may have been associated with a central burial. The radial fences are paralleled, in a different form, by the functionally similar, though earlier, divisions in the long barrows at Giants Graves, South Street and Beckhampton Road, all Wiltshire (Ashbee et al 1979). Radially arranged lines of stakes are not known under round barrows of the second millennium bc, but lines of stones subdividing a low cairn were found at Brenig 51, Denbighshire (Lynch 1975). They were interpreted by the excavator as administrative divisions like the fences at North Mains.

At the end of the first phase of construction, the North Mains mound was a massive ringbank surrounding an open central area. This structural arrangement can be widely paralleled. At Tregulland Burrow, Cornwall (Ashbee 1958) the second phase of this food-vessel-associated site saw the erection of a cairn-ring surrounding an area in which a cremation, accompanied by a plano-convex knife, was found. At Chippenham 5 (Leaf 1940) a narrow annular gravel bank, revetted on its inner face by a stake fence, enclosed a broad open area containing the semicircular post setting mentioned above. It is not, however, the author's intention to list all the available slight parallels, for better parallels are to be found rather closer to North Mains.

The early third millennium bc mound at Pitnacree in Strathtay (Coles \& Simpson 1965) took, at one stage in its development, the form of a penannular stone bank, possibly completed in the area of the entrance passage by a bank of mixed stone and soil; though this was not suggested by the excavators, if the bank was incomplete the entrance passage would be superfluous. The excavators pointed to the similarity of the form of the stone bank to the Clava ring-cairns, a comparison which can also be made for North Mains. The massive kerbing of the central open areas of the Clava type seem to have served, in part at least, a similar function to the completed wooden enclosure under the barrow. However, the later radical alterations to the shape of the mound, as at Pitnacree and North Mains, have not been found in the excavated Clava cairns. The central area at North Mains was c $7 \mathrm{~m}$ in diameter, very close to the average of $22 \mathrm{ft}(\mathrm{c} 6.7 \mathrm{~m}$ ) calculated by Burl (1972) for Clava ring-cairns but the ring-bank of the barrow was rather greater in diameter.

The excavation of the ring-cairn at Culdoich, Inverness-shire (Piggott, S 1955, 190) showed a cremation, deposited possibly on the pyre site, buried beneath the fill of the central area. This might be compared to the covering of the presumed central inhumations at North Mains with the embanking material of Period III, phase B. The practice in the smaller, perhaps derivative, ring-cairns, especially common in the NE, which also have instances of in situ cremation, may also be comparable.

The present obscurity of the age of the Clava ring-cairns and their precise relationship to 
the ring-cairns and recumbent stone circles of the NE allows only slight consideration of their possible relationship with Pitnacree and its possible derivative, the North Mains barrow.

Burl has placed the ring-cairns and recumbent stone circles in the earlier second millennium $\mathrm{BC}$ and the Clava cairns in the late third millennium, close to the period of construction of the North Mains barrow but rather later than Pitnacree, which might therefore be seen as an element in the development of both the Clava ring-cairns and the North Mains type of barrow. However, the wide distribution of ring-cairns, ring-banks and similar sorts of enclosed burial and ceremonial ritual monuments should remind us of the danger of a simple approach based on the comparison of structural features. However, the recent excavation of the class I henge at Balfarg, Fife (Mercer 1981) where one of the timber rings may have increased in height towards the SW has suggested an interest by their builders in the SW, an aspect noted already in the recumbent stone circles and in the Clava ring-cairns (Burl 1972). The associations of recumbent stone circles and ring-cairns in the NE has been discussed elsewhere (Ritchie \& MacLaren 1972; Kenworthy, J B 1972).

Parallels may be found for later elements in the mound, though whether they represent truly comparable activities is not clear. One or more fires associated with food vessel sherds were set on a platform defined by stakes on top of the barrow at Davidstow Moor, Cornwall (Croft Andrew 1948). At Amesbury G71, Wiltshire (Christie 1967) a hollow, also defined by a stakering, had been cut into or left in the top of the mound. The excavator noted that the surface of the hollow showed signs of burning. Whether either of these episodes was intimately associated with the building of the barrow, as was clearly the case at North Mains, is unknown. The Amesbury platform is dated to $1640 \mathrm{bc} \pm 90$ (NPL - 75).

Outer casings of stone and soil applied to barrows, as at North Mains in Period III, phase C (turf) and phase F (stone), are known on English barrows. At Ottersham, Cornwall (Dudley 1961) a subsoil layer was placed over much of the surface and a similar feature was found at Poole barrows I and II, Dorset (Case 1952). At Bevan's Quarry, Gloucestershire (O'Neill 1967) a stone sheath had covered the mound. Whether these features had a practical purpose, perhaps to prevent erosion, were entirely decorative, or had a more abstruse function is not clear. The stone covering at North Mains might possibly represent a later remodelling of the mound for a burial, since destroyed, at the top of the mound, under the circular stone setting.

The cupmarked stone, if it was originally set upright on the top of the mound, can be compared with the standing stone on top of the Pitnacree barrow (Coles \& Simpson 1965, 38); burial $\mathrm{K}$, the remnants of a disturbed cremation burial found in the disturbance pit, might also have been placed at the slab's foot. The practice of cremation burial with a standing stone is, however, too common to merit further comment in this paper (Ritchie, J N G 1974, 93; Barber 1978, 107).

\section{THE SECONDARY BURIALS}

Because of the disturbance of the mound surface the precise relationships of the burials to the mound and to each other could not be determined, although approximate termini post quos could be established for most: Burial A - period III, phase F; Burial B - IIIC; Burial C - IIID; Burial D - IIIC; Burial E - late IIIC; Burial F-IIIF; Burial G - late IIIC; Burial H - late IIIC; Burial J - late IIIC; Burial K - IIIC.

Burials $\mathrm{E}$ and $\mathrm{G}$, the two inhumations, surviving only in fragments, were both accompanied by food vessels (SF41; SF42). Burial E was a typical Early Bronze Age cisted inhumation broadly similar to burial $\mathrm{C}$ on the henge and to a great number of other examples in Scotland. It was built of ill-matched slabs and blocks, conforming to McAdam's general description of food vessel cists. The paving to the $\mathrm{E}$ and $\mathrm{N}$ of the cist, though serving to level the cist sides, may also have 
had a use during the burial ritual. Child burial in cists accompanied by food vessels (and other non-beaker grave goods) has been estimated at $25 \%$ of the total of the known examples (McAdam 1974, 40).

Burial $G$, an inhumation set within an apparently unlined pit partly covered by stone slabs, was similar to burial $D$ on the henge. As a form of burial in the third and second millennia bc it is poorly represented compared to cists. This imbalance is very probably the result of the greater ease with which stone-lined pits with capstones are found during ploughing and quarrying (Stevenson, J B 1975). The disc-type jet necklace which accompanied burial G can be paralleled in a similar context at Dalgety, Fife (Watkins 1982). The position and arrangement of the necklace would suggest that the body lay with its head to the $S$, facing $W$.

The rest of the burials were cremated deposits, cisted with the exception of $\mathbf{J}$. Nothing is known of the container used for $\mathbf{K}$. Only $\mathbf{B}$ and $\mathrm{C}$ were accompanied by recognizable artefacts. With the former were two burnt flints identifiable in traditional terms as knives, placed by association in the Early Bronze Age (SF107; SF108). The burnt, broken retouched flint tool with burial C (SF109) could not be identified as a tool type within the traditional morphology. As no charcoal was found with the burials the cleaning of the bones from the pyre must have been carefully done, suggesting that these sizeable pieces of flint were not only burnt with the bodies but placed deliberately with them in the cists.

Of the eight deposits of cremated bone five were multiple burials. Denston's most conservative estimates suggest that 25 individuals were buried in five cists, with the addition of the three individuals in $\mathrm{F}$, $\mathrm{J}$ and $\mathrm{K}$. This multiple deposition practice has not yet been found widely in this period.

The individual cremation burials were of adult females, though Denston has suggested that the sex identifications of burials $\mathrm{J}$ and $\mathrm{K}$ are uncertain. The other burials were mixed deposits of adult males and females, and children ranging from neonatal to adolescent. In each there was at least one adult of each sex. It is possible that the male and female adults, and the child and infant of burial B might represent part of, or a whole nuclear family. Burial A, which consisted of three adults, one certainly female, and a young child or fourth adult might also represent part of a single family. The female was over 30 , conceivably a grandmother.

The evidence of excavation shows that the deposits were thoroughly mixed prior to burial. It is possible to interpret the burials in a variety of ways. If the bodies were not burnt together then the cremated remains of the first to die may have been kept until sufficient had been gathered to allow, or to justify, the construction of the cist. If the bodies were cremated together then the burials might represent the disposal of those who died in a short period from one family or a larger settlement, perhaps as the result of virulent disease or a disaster of natural or human origin. The large proportion of children in burial D might suggest the death by disease of the more vulnerable members of the community. The rite in burial $\mathrm{C}$ differed in that several individuals were buried in the cist and some outside in the packing behind the slabs.

The cists varied somewhat in their design and construction. The polygonal cist of burial B was superficially similar to that containing burial $\mathrm{H}$. The remainder were of individual style, ranging from the massive, well built cist of burial $\mathrm{C}$ to the small, very roughly built cist of burial A.

Base slabs appeared in $B$ and $H$, with the addition of a single cupmark in the latter. However, the uncisted cremation (burial $\mathbf{J}$ ) was laid on a slab. $\mathrm{B}$ and $\mathrm{H}$ were multiple burials though the latter included no children. The methods used to cover both were similar; a ring of flags was laid round the edge of the cist, over the side slabs, leaving a space at the centre of the cist. This was covered by a second ring of flags (partly in B, wholly in H). A capstone was laid over this setting in $\mathrm{H}$. 
In burial $\mathrm{C}$ the floor of the cist had been compacted prior to deposition and in the cist of burial $\mathrm{E}$ a thin layer of gravel covered the bottom. The cist of burial $\mathrm{D}$ had been backfilled to a depth of $15-20 \mathrm{~cm}$ prior to the deposition of the cremated remains. There was no evidence of special preparation of the bottoms of the remainder of the graves.

The quantity of burnt bone found in each burial (excluding $\mathrm{K}$ ) varied from $585 \mathrm{~g}(\mathrm{~J})$ to $6760 \mathrm{~g} \mathrm{(C)}$. In the single deposits the weights recovered were $881 \mathrm{~g} \mathrm{(F)}$ and $582 \mathrm{~g}(\mathrm{~J})$. If the total weight in the multiple deposits is divided by the lowest estimate of the number of bodies the weight per body varied between $406 \mathrm{~g}(\mathrm{~A})$ and $949 \mathrm{~g}(\mathrm{H})$. The number of children in some of the multiple deposits would substantially lower the average weight per person and given some differences in cremation, collection and comminution practices, as well as in the individuals undergoing the process, it is likely that the burials are of the greater part of each individual's cremated remains. The low weight of burial $\mathrm{J}$, if not accounted for by different processing, can certainly be explained by its vulnerability, as an uncisted deposit, to disturbance. The pig and possible bos bones recovered from burial $\mathrm{C}$ may indicate the cooking or burning of animals during the rite, or the proximity of the pyre to a domestic site. Random inclusion of animal bone can be paralleled at Dalineun, Argyll (Ritchie, J N G 1972, 60).

Burials $B$ and $H$ lay relatively close together in the $S$ and SE parts of the mound where all but three of the cremations ( $C, J$ and $K$ ) were found. The two inhumations ( $E$ and $G$ ) lay close together at the bottom of the E flank of the mound, possibly suggesting that whichever one was placed first was marked in some way, allowing the second to be placed close by.

Burial C may have been inserted during the building of the mound, as may some of the others (supra $\mathrm{p}$ 239). Apart from the termini post quos provided by various phases of the mound, absolute chronologies for the burials cannot be provided; no suitable material was available for radiocarbon dating. Food vessel sherds were found in the Period III, phase B embanking of the central enclosure and complete food vessels were found in burials $\mathrm{E}$ and $\mathrm{G}$. The erection of the mound has been radiocarbon dated to the 16 th to 19 th centuries bc. The earliest food vessel dates at present available lie in the 18th century bc, perhaps the earliest date allowable for the insertion of the two food vessel inhumations and the IIIB sherds. Burial B is dated to a similar period by the two knives and burial $\mathrm{H}$ is similarly dated by its close structural resemblance to $\mathrm{B}$. The remainder of the cremations cannot be dated with any certainty. Their common multiple burial practice might suggest a rough contemporaneity with $B$ and $H$. Certainly the rite differs from that found in the late second or early first millennium pits to the $\mathrm{N}$ of the henge (supra $\mathrm{p} 187$ ). It is therefore possible that the inhumations and cremations were broadly contemporary, complementary aspects of the same tradition. Burgess $(1980,297)$ has suggested that cremation and inhumation were merely options within a single burial tradition, though perhaps showing some distinction in the standing of the subject(s). He has suggested that different status might be implied by the placing of a burial in a previously unused area rather than by re-using a site, and that beaker burials were very often in the former situation. If the North Mains barrow covered one or more individuals then the position of some of the later burials might be truly secondary, people not worthy of their own site or, conversely, people well qualified to gain a burial place in a prestigious area or, of course, entirely fortuitous. The traditional explanation, the mere attraction of a ceremonial site, as on the henge perhaps, might equally be considered.

\section{THE ARTEFACTS}

Most of the finds from the old land surface and the mixed area near the edge of the mound, where the differentiation between mound and surface was difficult, could have originated in any period up to and including the beginning of mound building or the deposition of the turf layer in 
Period III, phase C. It has been possible to group the finds in bands, between their earliest and latest possible periods of deposition.

The assemblage of flaked stone of find groups $1-3$ is very small and therefore adds little to the understanding of Periods I, II and III. The lop-sided arrowhead (SF53) is traditionally a later Neolithic artefact but it was impossible to say whether it, the other retouched piece (a scraper flake; SF54) and the three waste pieces, had been dropped on the site or had found their way there with midden material deposited as fertilizer. The occurrence of the pitchstone (SF50) provides the earliest firm date for the use of this material on all the North Mains sites, as none of the pieces on the henge was found in undisturbed contexts. The flintwork may represent: (i) debris from the sharpening and altering of tools, (ii) possibly flakes used without retouching, and (iii) material incorporated into the body of the mound in the spoil used in its construction.

Though much of the pottery assemblage shares with the flaked stone the problems of derived context, the greater capacity for the exercise of individual aesthetic or traditional desires on ceramics allows more valuable comparisons with other pottery assemblages. Cowie has described the general featurelessness of the barrow pottery assemblage. However, the appearance of possibly earlier and later (p 216) Neolithic pottery confirms that the area had been in use for some time prior to the erection of the mound and that the sub-OLS features of Period I reflected settlement activity of that earlier period.

Few sherds worthy of comment were recovered from the body of the mound. The possibly late Neolithic bipartite vessel fragments from the old land surface have been mentioned. The other sherds could be assigned to the very general category of 'Western Neolithic' pottery, probably settlement debris from the topsoil redeposited during the construction of the mound. Likewise the beaker material found in the upper part of the mound and the food vessel sherds found in Period III, phase B deposits might well have originated in pre-mound activity.

The perforated disc, apparently a 'spindle whorl', was found in an undisturbed Period III, phase $\mathrm{C}$ context and can be dated at the latest to the mid second millennium bc, and is therefore one of the earliest known examples in Scotland. The circumstances of discovery of many perforated discs of this type leave much to be desired; most are unassociated chance finds. Many may therefore be contemporary with or earlier than the North Mains example. The disc is very similar to a spindle whorl from Balcraig in Wigtownshire (National Museum of Antiquities of Scotland: BE197) which combines on one surface radial scored lines and a line concentric with the perforation. Though radial lines and single or multiple concentric rings are common decorative features they occur together relatively infrequently. However, the poor dating evidence for most whorls, including the Balcraig example, and the simplicity of the decorative styles make comparisons uninformative. A perforated clay disc, interpreted as a spindle whorl, was found in a late Neolithic context at Durrington Walls (Wainwright \& Longworth 1971, 189). A possible further example was recovered at Carnaby Top Site 20 (Manby 1974, 35, fig 11, 13). A perforated disc ('drill weight') was found in the tomb at the Ord North, Sutherland (Sharples 1981).

The disc-bead jet necklace accompanying burial G (SF113) can be paralleled in complete necklaces or small deposits of beads. Simpson (1968) has listed examples associated with food vessels; a shale necklace of similar design has since been found with a degenerate beaker in cist 4 at Dalgety (Watkins 1982).

The stone artefact found in the ditch (SF112) may be interpreted as a roughout for a hammer or macehead on which the perforation has not been completed, or better, perhaps, as an unusual portable cupmarked stone. As it was found in the ditch its relationship with the mound is not clear. 


\section{The ring-ditches}

\section{INTRODUCTION}

Some $280 \mathrm{~m}$ to the ENE of the henge lay the third site threatened by the landing strip development. On the only aerial photograph of the site (RCAMS PT 5468) two small cropmark rings and some ill-defined linear features were visible. A total of $426 \mathrm{~m}^{2}$ was opened. The $\mathrm{E}$ portion of the site was archaeologically sterile and is therefore not illustrated on fig 61 .

\section{DESCRIPTION}

\section{LINEAR FEATURES}

On excavation these were proved to be of natural origin.

RING-DITCH 1 (figs 61, 62; pl 16)

A single circular ditch between 0.2 and $0.4 \mathrm{~m}$ deep and $0.8-1.1 \mathrm{~m}$ broad enclosed an area between 4 and $4.7 \mathrm{~m}$ in diameter. The ditch was sectioned axially and laterally to try to find traces of any structure which may have been set in it; no evidence for any structure was recovered. The ditch had been dug, in $75 \%$ of its circumference, to the level of the natural yellow-green silt which, as on the barrow, underlay the gravel. The result was to give a rather lurid appearance to the ditch bottom. Within the enclosed area was a single pit (F1) which was apparently cut by the ring-ditch.

\section{RING-DIrCH 2 (figs 61, 62)}

To the SSW of ring-ditch 1 a second, smaller circle was excavated. The ditch was $0.05-0.2 \mathrm{~m}$ deep, $0.3-0.6 \mathrm{~m}$ broad and enclosed an area between 2.3 and $2.8 \mathrm{~m}$ in diameter. The NE segment of the ditch was the shallowest. No trace of a structure was found in the ditch except at the SW where a single possible post-pipe was noted. A small quantity of cremated bone (cremation catalogue 3) was found in the ditch fill, close to F3, a feature within the enclosed area, apparently cut by the ring-ditch.

\section{OTHER FEATURES}

F1 (fig 62) This small pit lay at the $\mathrm{E}$ side of the area enclosed by ring-ditch 1 and had apparently been cut by it. It measured c $0.4 \mathrm{~m}$ in diameter and $0.2 \mathrm{~m}$ deep. It was filled with charcoal flecked loam and stones which showed clear signs of fire reddening.

F2 (fig 62) A small pit with a uniform loam fill, measuring c $0.2 \mathrm{~m}$ in diameter and $0.17 \mathrm{~m}$ deep.

F3 (fig 62) This feature lay at the SW edge of ring-ditch 2 which apparently cut it. It was filled with a silt loam covering a layer of charcoal (radiocarbon dated to $2690 \mathrm{bc} \pm 65$ (GU-1546)) and contained $6 \mathrm{~g}$ of burnt bone (cremation catalogue 3). Four sherds of pottery were recovered from the feature (SF2, 3, 4, 5). The pit was bottomed on a natural layer of green silt.

F4 (fig 62) Immediately to the SW of ring-ditch 2 lay a pit measuring $\mathrm{c} 0.65 \mathrm{~m}$ in diameter and $0.55 \mathrm{~m}$ deep. It was filled with charcoal-stained silt loam and gravel. The outer edge of the ring-ditch appeared to kink as it passed this pit.

F5 (fig 62) This posthole lay to the $E$ and $S$ of ring-ditch 1 . It was $0.4 \mathrm{~m}$ in diameter and $0.4 \mathrm{~m}$ deep. The post-pipe measured $c 0.2 \mathrm{~m}$ in diameter and was filled with a stony loam surrounded by stone packing.

\section{THE POTTERY FROM THE RING-DITCHES AT NORTH MAINS}

\section{T G Cowie}

\section{INTRODUCTION}

The pottery recovered from the ring-ditch area consists of 10 sherds. Most of the pieces are featureless and apparently unrelated body sherds, representing perhaps as many as eight vessels. The published 


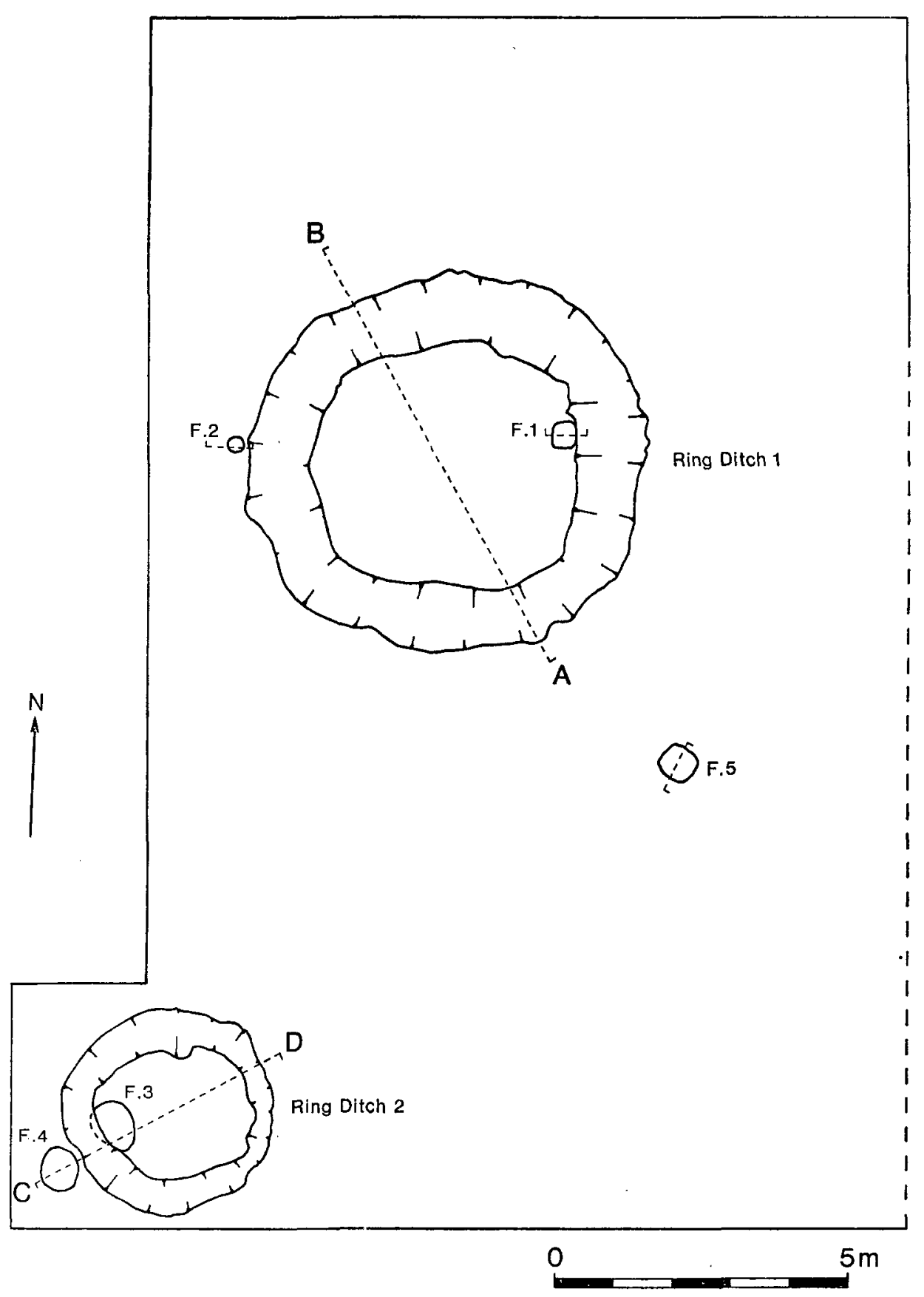

FIG 61 Ring-ditches site plan 


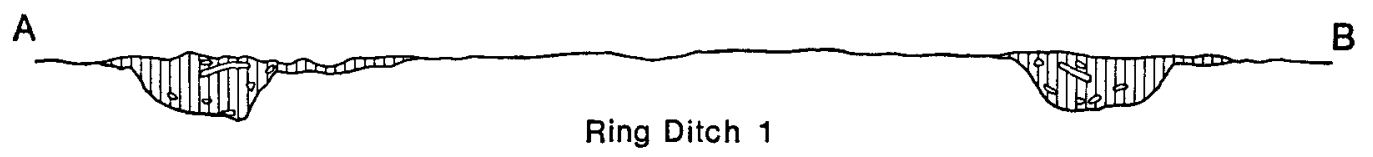

Ring Ditch 1

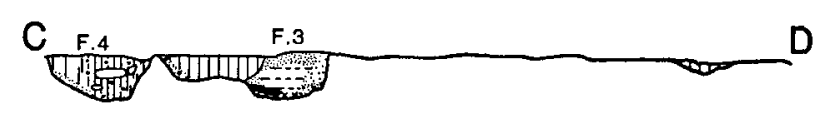

Ring Ditch 2

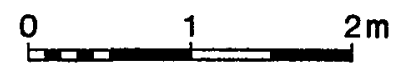

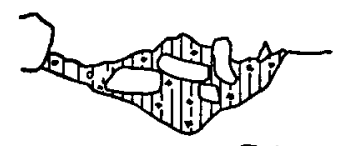

F.1

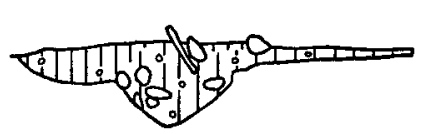

F.2

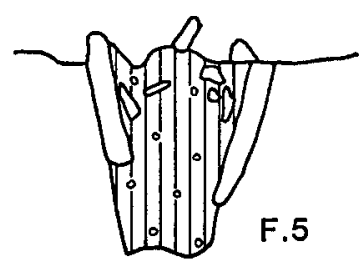

$1 \mathrm{~m}$

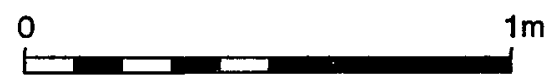

FIG 62 Ring-ditches: sections of ring-ditches 1 and 2, and other features
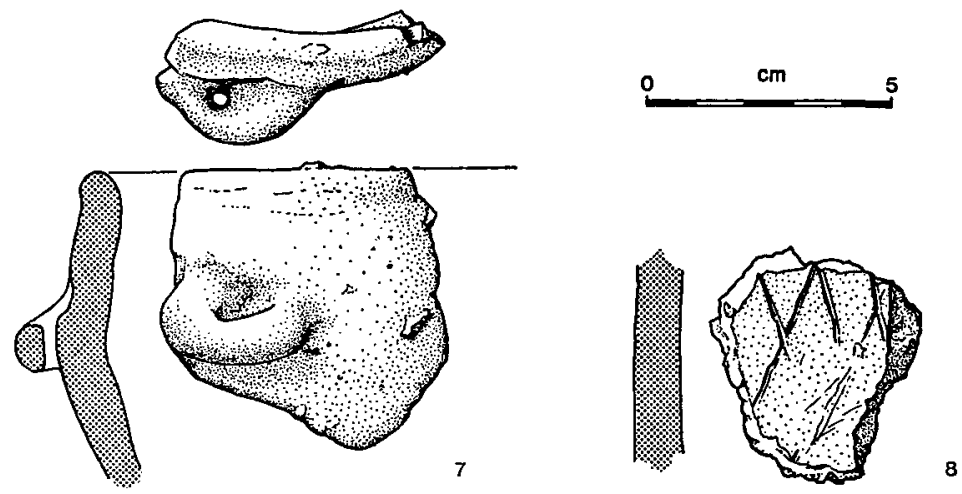

FIG 63 Ring-ditches pottery

catalogue provides summary details of these sherds, and is set out in the format adopted for the assemblages from the henge and barrow (see p 155 and p 210 above).

\section{CATAlogue}

Ring-ditch 1/Ploughsoil

SF1 Two body sherds, almost certainly joining; fabric J; relatively unabraded. 
F3

SF2 Body sherd, possibly from on or near shoulder of vessel; fabric K; relatively unabraded.

SF3 Body sherd; fabric K; burnt; abraded.

SF4 Body sherd, possibly from shoulder of vessel; fabric $\mathrm{K}$; relatively unabraded.

SF5 Body sherd; fabric K; relatively unabraded.

\section{F4}

SF6 Body sherd; fabric $\mathrm{K}$; possible bone or stick impression on external surface; relatively unabraded.

SF7 (fig 63) Rimsherd with perforated horizontal lug, c $30 \mathrm{~mm}$ below rim; probably sherd from a simple bowl, with rounded rim; rim diameter c $180 \mathrm{~mm}$; generally light brown to reddish yellow; fabric K; scorched?; abraded; surfaces fragile and flaky.

SF8 (fig 63) Body sherd; position and orientation uncertain; internal surface and core black, exterior reddish-brown; fabric K; on external surface, oblique slashed lines c $15 \mathrm{~mm}$ long, with more lightly incised lines meeting and overlapping these to form a rough zigzag; relatively unabraded; organic encrustation on internal surface.

SF9 Body sherd; possibly from same vessel as SF6; relatively unabraded.

\section{DISCUSSION}

The pottery from the ring-ditch area consists of a group of largely featureless body sherds, and in nearly every case the position of the sherds on the vessel and frequently their orientation are uncertain or indeterminate. SF2 and SF4 may each be from the region of the shoulder, but little light is thrown on the types of vessel in question. SF7, however, is a more significant sherd, representing a portion of the upper body of a lugged vessel. Although the overall shape is uncertain, the affinities of this pot clearly lie with the bowl forms of the Western Neolithic tradition.

The assemblage from the site is relatively homogeneous in terms of the fabrics present: with the exception of two sherds (SF1) all fall into fabric group K, distinguished mainly by the presence of very fine to coarse muscovite and biotite mica. There is in this case no reason why the material should not represent a small closed group of Neolithic pottery. In the presence of featureless body sherds, the minimum number of vessels represented cannot be gauged with any accuracy, but except where noted otherwise, the sherds appear to be unrelated parts of different pots. With the exception of two sherds (SF1) the pottery from this area was recovered from the filling of one feature, namely F3. In this respect the pit group contrasts with the fragmentary material from the barrow, there interpreted as an almost wholly derived assemblage, and the contrast is perhaps reinforced by the relatively unabraded condition of the sherds presently under discussion. One possible bone or stick impression on SF6 is too insignificant to merit more than passing reference, and decoration is present definitely on only one of the sherds, SF8, a body sherd of uncertain position and orientation, with traces of an incised pattern on the exterior.

\section{THE ASSEMBLAGE OF FLAKED AND OTHER STONE FROM THE RING- DITCHES AT NORTH MAINS}

\section{R Wickham-Jones}

The collection of flaked stone from the ring-ditches has been catalogued but as it comprises only six pieces no detailed information may be presented. Both flint and quartz are represented although only the flint may be said with any certainty to have been flaked by man. As well as the two small flakes of flint there are two retouched pieces. One, SF15, was broken after manufacture but there is evidence of macroscopic edge damage across the break that might indicate its reuse as a scraper. The other piece, SF10, is bifacially retouched and has not been broken but it is very small and does not conform to any pre-existing morphological type. It is possible that it is still an unfinished state, a preform, perhaps, for a projectile point, but there is no definite evidence for this and the small size would tend to argue against it. As it has survived this assemblage does not add to the picture of the use of stone at North Mains as previously constructed although it does fit within it. 
CATALOGUE

(See notes on p 167)

Ring-ditch 1

SF10 (fig 64) Retouched piece. Cortical flake; white; trapezoidal plan; irregular invasive retouch over both ventral and dorsal faces; $17: 15: 03 ; 121^{\circ} ; \mathrm{r} 21^{\circ} ; \mathrm{d} 26^{\circ} ; \mathrm{p} 25^{\circ}$; bifacially retouched piece.

F3

SF11 Primary flake (quartz).

Ploughsoil

SF12 Secondary chunk (quartz).

SF13-14 Inner flakes.

SF15 (fig 64) Retouched piece. Inner flake; pale grey; corticated; patinated; broken; proximal surviving; artificial platform; straight sides broadening from straight proximal to broken distal; small irregular edge retouch truncated by breakage on both sides; macroscopic edge damage across distal

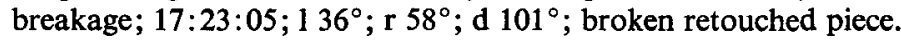

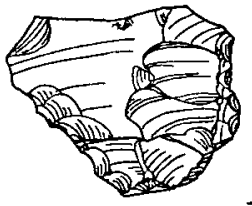

15

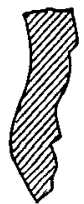

FIg 64 Ring-ditches flaked stone (scale 1:1)
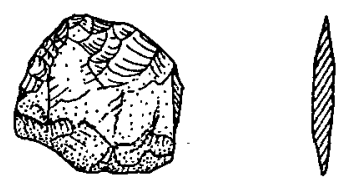

10

SF16 Hammerstone. Quartz; Irregular rounded shape; $61: 46: 47$; weight $195.5 \mathrm{~g}$; both ends blunted by battering; three small flakes removed from the broader end; areas of damage: $29: 32$ and 29:22; a small amount of isolated damage down each side. This stone would serve as an excellent hammer in the manufacture of stone tools but it would also be possible to use it for many other tasks.

\section{BURNT BONE FROM THE RING-DITCHES AT NORTH MAINS}

\section{R Powers}

\section{CATALOGUE}

\section{Ring-ditch 2}

1 Rolled fragment of dense white bone of triangular section, probably the anterior aspect of a tibia. Species indeterminate.

2 Sufficiently like 1 to be part of the same bone splinter; but no contact. Flatter, broader but still triangular.

F3

3 White, thin-walled long-bone. The best preserved fragments could be from the distal diaphysis of a human radius but are not diagnostic. Two pieces were reconstructed from three fragments each. Though this group might derive from human cremations the probability is that 1 and 2 derive from a large quadruped, perhaps bovid.

\section{DISCUSSION}

The two pits F1 and F3 are proveably earlier than ring-ditches 1 and 2 respectively. F4 is apparently deliberately avoided by ring-ditch 2 and is therefore probably earlier, though still 
visible or marked in some way when the ditch was dug. There is no evidence for the date of F2 or F5. As the two ring-ditches are similar structures they are probably broadly contemporary.

It is suggested that the ring-ditches were quarries for low barrows which, with the possible addition of turf or field-stone, covered the inhumations probably represented by the phosphate distributions ( $\mathrm{p} 269$ ). Whether the burial in F3 and the activity refiected in the fill of F1 are closely associated with the ring-ditches could not be determined by excavation. However, the features found might best be considered as a single pair of barrows with associated burial and ritual represented by F1, F3, F4 and the high phosphate concentrations within the enclosures. The pottery in F3 probably forms a closed group, unlike the remainder of the material, which is likely to be from prior surface scatters. The cremation can therefore be placed securely in the later Neolithic. Charcoal from the pit (species Alnus, Betula, Corylus) has been radiocarbon dated to $2960 \mathrm{bc} \pm 65$ (GU-1546). This date provides a terminus post quem for ring-ditch 2 .

The ring-ditch might be best compared with the Neolithic round-barrow and ring-ditch tradition described by Kinnes (1979). Many ring-ditches have been found by aerial photography in Scotland in recent years. The excavation of a ring-ditch enclosing a beaker-associated grave at Newmill (Watkins \& Shepherd 1980) suggests that some may be barrows of the late Neolithic/ early Bronze Age. Many of the cropmark sites seem to have single central or multiple pits within them. The North Mains evidence confirms that low barrows surviving as ring-ditches might originate earlier in the Neolithic in Scotland. The major difference in rite, apart from the apparent absence of grave goods, seems to be the lack of a burial pit deep enough to survive modern ploughing, though burial on the relatively unaltered old land surface was found at both, later, barrows at Broughton Knowe (MacLaren 1967; Ward 1970). It has been calculated, assuming a topsoil depth of $25 \mathrm{~cm}$, that the ditch of ring-ditch 1 would yield a little over $8 \mathrm{~m}^{3}$, that of ringditch $2,1.87 \mathrm{~m}^{3}$. Barrows, conical for the sake of simplicity, of height 1.03 and $0.79 \mathrm{~m}$ could be postulated for the two ring-ditches. More substantial mounds could be built using some field stone in addition, as on the main barrow.

\section{General discussion papers}

General contributions on the Pottery (including Kenworthy's fabric analysis summary), the Flaked Stone, the Origin of the Pitchstone, the Radiocarbon Dates, the Soils, the Phosphate Surveys, the Pollen and the identification of Stone Types follow. They are followed by the excavator's summary and conclusions.

\section{THE POTTERY FROM NORTH MAINS: GENERAL DISCUSSION}

\section{T G Cowie}

The details of the pottery from the three excavated sites have been described above (pp 155, 210, 243): in the discussion which follows all three assemblages are considered as a whole and an attempt is made to set them in their wider context. However, it is worth prefacing this discussion with a brief resumé of the material from each site in turn.

THE HENGE

The pottery from the henge includes material from a variety of contexts and ranges from complete vessels accompanying burials to an assortment of fragmentary pottery, the circumstances of deposition of which are uncertain. It is likely, however, that the presence of the fragmentary 
and largely featureless sherds of 'Western Neolithic' pottery in the assemblage reflects the existence of scattered, originally domestic, pottery lying in or on the ground surface eventually disturbed by the builders of the henge. Some of the sherds in this residual scatter appear to have been sealed by the construction of the bank; others appear to have been incorporated, perhaps quite fortuitously, into the fills of features where, on a site truncated by plough damage, there is potential for biased survival of sherds. None of the ceramic material is directly associated with structural elements of the henge, and even the coarse beaker sherds in the upper fills of two of the large post-pits reflect activity on the site subsequent to the decay of the timber posts. The remainder of the pottery consists of seven more or less complete vessels recovered from funerary contexts: these, a late beaker, three food vessels, a collared and an enlarged food vessel urn, and another urn of related but unusual type provide a significant new group of funerary pottery from a single site in Scotland. In the absence of stratigraphy their sequence of deposition remains uncertain. By analogy, however - for instance with the sequence observed at Cairnpapple Hill (Piggott, S 1948) - it would be traditionally suggested that the inurned cremations succeeded the inhumation burials; in the current state of knowledge any attempt at further precision would not be justified (Burgess 1980).

\section{THE BARROW}

The pottery assemblage recovered from the barrow primarily consists of largely featureless sherds. With the exception of two food vessels deliberately placed in graves set in the mound, and a late Neolithic bipartite vessel from an ambiguous context near its periphery, there is little or no reason to suspect deliberate deposition of pottery during the construction of the barrow. The context, the variation in degree of abrasion and the fragmentary and largely featureless nature of the remainder of the assemblage tend to suggest the sealing or incorporation, during the mound's construction, of scattered, possibly originally domestic 'Western Neolithic' pottery lying in or on the ground surface eventually covered by the barrow, or in the soil used in its construction. This may also explain the presence of two decorated pieces, a sherd with comb impressions and a tiny sliver, both tentatively identified as beaker, for there is nothing to suggest that their incorporation on the site is anything other than accidental. During the primary stages of barrow construction (Period III, phase B), pottery in the food vessel tradition was in currency, as demonstrated by a rimsherd from the gravel and silt infill of the central enclosure. This ceramic tradition was to be represented again on the site by the two food vessels, already mentioned above, deposited as accessory vessels to interments in secondary graves (burials $\mathrm{E}$ and $\mathrm{G}$ ). Finally, parts of one or more wheel-turned vessels appear to have arrived on the mound in more recent times in the course of unrecorded medieval activity.

\section{THE RING-DITCH AREA}

Fabric analysis and the circumstances of recovery of the material in relatively fresh condition from a unitary context tend to suggest that the pottery from F3 near Ring-ditch 2 represents a closed pit group. Part of an unusual lugged bowl and a decorated body sherd are on that basis sufficiently diagnostic to allow attribution of the group as a whole to the 'Western Neolithic' tradition.

\section{NEOLITHIC POTTERY}

(i) Western Neolithic or Grimston/Lyles Hill series

Smith has argued $(1974,106)$ that the use of certain generic terms for earlier Neolithic 
pottery, such as Neolithic A, Windmill Hill or Western Neolithic ware, is no longer appropriate and has proposed that these should be abandoned in favour of a terminology based on the recognition of ceramic traditions named after type sites; where possible these broad groupings may be refined into more precisely defined styles (cf Scott 1977, 26). Smith's Grimston/Lyles Hill series would thus embrace most of the pottery from East/Central Scotland formerly categorized under the heading of 'Western Neolithic', although Scott has recently queried the validity of relating the two, preferring to view the Grimston and Lyles Hill styles as more or less discrete strands in the development of Scottish Neolithic pottery (1977, 36-37). In the case of the Neolithic assemblage from Strathallan many of the sherds are identifiable as Neolithic only on the basis of their fabric, and although there are exceptions where the wider relationships of the pottery are not in doubt, the limitations imposed by this as a source of evidence are such that a specific attribution is frequently untenable, and could at worst be misleading, at least in the absence of more detailed analyses of Neolithic ceramics. It is felt, therefore, that there is still a need for a nonspecific or 'blanket' term to distinguish at a basic level the products of the characteristically undecorated, or only lightly decorated, bowl tradition of the earlier Neolithic, from the impressed decorated wares of the later Neolithic. In the present report, therefore, the writer has, with reservations, retained as a piece of deliberate conservatism the term 'Western Neolithic' in this traditional generic sense: the term carries no detailed implications for the range of vessel shapes, but is instead intended only as a signpost towards the much wider corpus of British earlier Neolithic pottery with which the Strathallan assemblage might be expected to share general affinities. On balance, much of the Neolithic pottery would indeed fall into the Grimston series in the flexible sense defined by Smith $(1974,108)$ but many of the sherds cannot at present be assigned unequivocally to a particular tradition, far less a specific style. This being the case, there is arguably little point in replacing one portmanteau term (or, more colloquially, 'dustbin' category) with another, and it would seem preferable in this instance to retain the more specific terminology proposed by Smith and Scott for more readily classifiable assemblages. It is to be hoped that advances in the study of Scottish Neolithic pottery will in time permit greater definition of the material.

It is unfortunate that the Strathallan assemblage is so featureless, for its size makes it a valuable addition to the currently limited corpus of Neolithic pottery in eastern and central Scotland. It is also unfortunate that the material is almost wholly derived, as this rules out its treatment as a closed group, except in the most limited way in the case of the material sealed, in the strict sense, by the barrow. There is a little evidence to suggest that the small collection of sherds from a pit at the ring-ditch site may be a more coherent group, but the sherds concerned have little in common with the material from the other two sites. Indeed, it is noticeable that with only one or two exceptions (eg fabric group G, which includes the sherds SF24 from the henge and SF39 from the barrow) there is little detailed correlation in the fabric represented on the three sites excavated, reinforcing the potential danger of attributing relatively featureless sherds to anything other than a general 'family' of wares. The reasons for the apparent distinctions between the barrow fabrics, on the one hand, and the henge and ring-ditch site on the other, may be due to archaeological biases: being a 'sealed' unit of larger amounts of anciently deposited soil, the barrow will have tended to favour the survival and hence recovery of greater quantities of sherds, and thus the henge material may simply not be representative. On the other hand, more complex spatial or chronological factors could be invoked to account for these differences, but until our knowledge of the local Neolithic fabrics is advanced, it is not possible to do much more than note these variations. In summary, there appears to have been a general scatter of Neolithic pottery, originally derived from a domestic context or contexts, over all the areas 
subsequently disturbed by the builders of the henge and mound, but possibly much more extensively over the terrace on which the sites lie. The dispersal of broken pottery along with other domestic refuse into cultivated areas in the course of manuring is one explanation for this sherd scatter, although the presence of at least one possible contemporary pit (ring-ditch site, F3) indicates that some of the original contexts of the pottery need not have been far away.

Turning to the sherds in more detail, the marked absence of rims and other diagnostic features renders detailed comparison with the material from other sites difficult. However, taking the more informative pieces in turn, it is possible to offer a tentative reconstruction of at least some of the original components of the Neolithic assemblage. The small rimsherd (barrow) SF13, is of a simple externally expanded form without precise parallels in other assemblages in the region though akin to a rolled rimsherd from the Catstane cemetery near Edinburgh (Henshall in Cowie 1978b, 197, SF11). The range of vessel shapes which might be associated with this relatively simple rim type, is illustrated, for example, by the Luce Sands material (McInnes 1964, eg nos $17,18,54)$; this also emphasizes the danger of attempting detailed interpretation of the original bowl form on the basis of a single small sherd.

A shouldered bowl of uncertain form is perhaps also represented by the group of sherds (barrow SF23) in a dark grey gritty fabric; the unusual building features of this vessel are worth mentioning for, if found alone in an abraded condition, the false rim (b) might well be interpreted as a flattened, externally expanded, rim; whereas, it seems clear that flaking of the clay has resulted in the loss of portions of a rounded slightly everted rim of the type represented by the surviving rimsherd (a). It is unfortunate that the original shape of the vessel cannot be reconstructed with certainty: if the rounded profile of the body sherd (c) really does reflect the presence of a rounded shoulder, this would mark out the pot as a relative rarity among Scottish Neolithic pottery where carinated vessels are virtually the norm (cf McInnes 1964, 43).

The potential for misrepresentation also exists in the case of (barrow) SF19, here drawn and tentatively catalogued as the rim of an open bowl with relatively straight sides and flaring slightly flattened rim. However, it is possible that abrasion of a construction joint may have presented a misleading impression : the possibility exists that the sherds may be from the collarof a carinated bowl which would not be out of keeping with the relatively compact, thin fabric of the piece.

Certainly the presence of shouldered vessels in the assemblage is demonstrated by two of the three lugged sherds recovered from the barrow: the original form of the lug on the shoulder sherd (barrow) SF1 is uncertain but the proportions of the sherd, and indeed the general quality of the fabric, suggest that these sherds represent parts of a vessel comparable (at least in the general outline of the shoulder) to the carinated bowl from Cultoquhey near Crieff, also in Perthshire (NMAS EO 1002; Henshall 1972, 475-6, fig 306). The atypical fabric of the Cultoquhey vessel was noted by Henshall $(1972,172)$, but in the light of the broadly similar fabrics of group B at Strathallan this may no longer need to be seen as an exceptional feature. The rounded lug (barrow SF3), unfortunately also imperfect, would likewise not be out of place on the carination of a shouldered bowl, while a third lugged sherd from the barrow (SF45), consisting of a simple pinched up knob (cf Piggott, S 1931, 77 (type al)), might typically occur on the upper body of a simple bowl.

Turning to the henge, only two rims are present in the possible Neolithic material from that site: one (henge SF26) is slightly inturned and is perhaps best seen as part of a slightly closed bowl, possibly similar in proportions and form to the simple though decorated bowl from Craig in Auchindoir, Aberdeenshire (Callander 1929, fig 40, no 4; Henshall 1983, cat no 15). The other rimsherd (henge SF1) in a dark grey coarse fabric is only tentatively attributed to the Neolithic assemblage. A similar, though thinner rim form occurs among the Group I coarse ware recovered 
from Croft Moraig (Piggott, S \& Simpson 1971, 10, P1 IV, 4) but there is no similarity in fabric. While reserving judgement on the sherd in question, the point may nevertheless be made again that, as for example at Croft Moraig and Easterton of Roseisle (Henshall 1983) broadly contemporary assemblages may include a range of wares ranging from fine, readily identifiable fabrics and forms to coarser wares which may be less amenable to classification, and hence identification, when recovered in isolation.

Finally some mention must be made of the two most significant pieces of pottery from the pit group recovered from the ring-ditch site. Both the rimsherd (SF7) and the decorated body sherd (SF8) lack close parallels in the known Scottish Neolithic material. The vertically perforated form of lug on SF7 is of a type occasionally represented in southern England (eg at Windmill Hill (Smith 1965, 50 fig 22, p 108)) but elsewhere in the British Isies it is very rare (Henshall 1972, 172). Henshall notes only three examples of vertically perforated lugs on Neolithic vessels from Scotland: two Achnacree bowls; one of them the vessel from Cultoquhey already mentioned, have perforations through minimal lugs on the carination, while a single body sherd recovered from the old ground surface under the Pitnacree barrow has an elongated vertically perforated lug set on the angle of the carination (Henshall 1972, 101, 172; Coles \& Simpson 1965, 42, fig 4.2). The form of vessel represented by the Strathallan sherd is uncertain but the likeliest orientation might suggest a somewhat globular bowl. The fabric of this rimsherd, and indeed that of several of the featureless sherds from the same context, is unlike the other Perthshire Neolithic wares examined by the writer: in texture and composition the fabric instead invites comparison with the fine but rather friable buff-grey ware of one of the carinated bowls from Cairnholy (Piggott \& Powell 1949, 118, no 1; Henshall 1972, 440). The decorated sherd (ring-ditch) SF8 also lacks ready comparison in the relevant Neolithic material from Scotland; in the Grimston/ Lyles Hill series, certainly, incised decoration is extremely rare. While the position of the ornament is uncertain on the Strathallan sherd and the arrangement of the decoration differs in detail, comparison may tentatively be made with the vertical incisions on the external surfaces of two, as yet unpublished, Neolithic rimsherds recovered from pits at Douglasmuir, Angus (Kendrick, pers comm). On one of these the incisions slant and overlap in places in a manner reminiscent of the Strathallan sherd. Both of the sherds from Strathallan just discussed thus possess exceptional features which set them apart from the Grimston/Lyles Hill series and perhaps hint instead at much more distant southern English connections, for example with decorated styles of the earlier Neolithic (Smith 1974, 108-11). Indeed, the discovery of such sherds invites the further gloomy reflection that the current limited corpus of Neolithic pottery from eastern and central Scotland may not be wholly representative, and might even be misleadingly incomplete.

Nevertheless, the assemblage adds considerably to the available groups of Neolithic material from east/central Scotland, of which the finds from Pitnacree (Coles \& Simpson 1965) and Croft Moraig (Piggott, S \& Simpson 1971) are locally the most important. Apart from the individual points of similarity already mentioned, sherds of the Strathallan fabric groups B-E may be compared, on visual examination at least, with examples of all three fabrics recognized at Pitnacree, and Strathallan fabric F compares even more closely with the Group II fabric at Croft Moraig. The other Neolithic fabrics recognized in the Strathallan assemblage offer fewer points of comparison, although mention has already been made of the broad similarity between sherds of fabric B from the barrow (SF1) and the vessel from Cultoquhey, and the general likeness of the fabric $\mathrm{K}$ material from the ring-ditch site, to a carinated bowl at the geographically rather remote site of Cairnholy in Kirkcudbrightshire. The range of fabrics encountered recalls the variation present in other assemblages (eg at Croft Moraig, commented on in Piggott, S \& Simpson 1965, 10-11), and reinforces the problems of attempting classification in the absence of larger quantities 
of diagnostic material, both on the site in question and from other sites for comparative purposes. Other sites in the general geographical region, besides those already mentioned, which have produced similar Neolithic pottery, (particularly in the distinctive slightly speckled micaceous ware similar, on visual inspection, to Strathallan fabric group F) include the Catstane, Midlothian (Henshall in Cowie 1978b, 197), Barbush Sandpit, Dunblane, Stirlingshire (unpublished) and Bantaskine, West Lothian (Callander 1929, 33, 56-7).

In general terms then, the Neolithic pottery from the Strathallan sites appears to represent a variety of vessels ranging from coarse, thick-walled vessels to thinner-walled vessels in fine wares offering more ready comparison with other sites. Several of the sherds point to the presence of carinated or even round-shouldered bowls while open bowls may also have been present. However, the circumstances of deposition of the pottery dictate that the assemblage cannot, in any but the most general way, be considered a contemporary group, for the material can really only be construed as a random scatter quite conceivably deposited at various times throughout the long currency of the potting traditions concerned. On current evidence, radiocarbon dating allows a date range at least throughout the first half of the third millennium bc. Indeed, to end on a cautionary note, the possibilities for admixture of quite unrelated groups of material must render provisional the Neolithic attribution of some of the featureless material.

\section{(ii) Late Neolithic}

Late Neolithic activity in the area subsequently occupied by the barrow is attested by the discovery near its eastern edge of sherds of a distinctive type and fabric (barrow SF21). As only portions of the pot are represented, the profile of the vessel is slightly uncertain, but sufficient remains to suggest a bipartite vessel, with profuse finger-pinched decoration, on and perhaps confined to, its upper part. Comparison may be made between this and the pottery recovered from a pit at Brackmont Mill, Fife: although there is no detailed correlation of the rim form, wall thickness or fabric of the sherds, it is possible to point to the similarity of the method of application and apparently the general arrangement of the decoration (Longworth 1967, 67-75 nos 3, 20 and particularly $\mathrm{pl} \mathrm{V,20)}$ and the presumed biconical shape and general proportions of the vessels (ibid, nos 1,3). Longworth suggested that heavy fingerpinched and rusticated surfaces might reflect the influence on local late Neolithic wares of beaker potting traditions, pointing to their widespread occurrence on the coarse wares on beaker domestic sites in the S (ibid, 73). The Brackmont group has for long stood in relative isolation in eastern Scotland and the Strathallan vessel provides a useful addition to the limited body of late Neolithic material from the region, while at the same time extending the known find spots of impressed ware inland to balance, in part, their present predominantly coastal, and surely biased, distribution (Smith 1974, 117). The vessel just discussed, and, more strikingly, the single sherd of a different pot found in the same group of material (barrow SF22) have rim forms reminiscent of the simple internally bevelled rims of many northern food vessels: it is worth recalling that Longworth $(1967,74)$ stressed the contribution that local forms of late Neolithic pottery are likely to have made to the traditions of the full Early Bronze Age. A radiocarbon date range spanning the later third and earlier second millennia bc might be applicable on the basis of the two dates from Grandtully for features containing 'Neolithic B' pottery (1970 bc \pm 100 (GaK 1396); and $2130 \mathrm{bc} \pm 190$ (GaK 1398) and not as quoted in McInnes 1969, 28).

\section{(iii) Beaker}

The beaker pottery from the Strathallan sites includes two weathered sherds from the barrow and a more representative group of material from the henge. It has already been suggested 
( $p$ 249) that the presence of the two pieces in soil incorporated into the barrow may be fortuitous, and in neither case do the small surviving fragments throw light on the type of the vessel concerned. The comb lines on (barrow) SF40 could be part of either a zone of horizontal lines or a single filled motif; similarly, little can be gleaned from the vague combination of light grooving and fingernail impressions which survives faintly on a fragment (barrow SF15) so small that its very identification as beaker ware is tentative.

The beaker pottery from the henge lends itself more readily to comparison. The virtually complete vessel (henge SF20) from burial F is rather irregular in shape with a slightly oval rim, only a slight waist and, in consequence, rather squat overall proportions. The loss of shape is complemented by the slackness of the manner and arrangement of the decoration which consists of roughly incised horizontal lines and chevrons in three zones. These features invite comparison with other typologically late beakers in the north: examples include vessels classified as S4 by D L Clarke (1970) which would all fall into the Step 7 stage in the modified scheme of Lanting and van der Waals (1972), for example the beakers from Linlathen, Angus (Clarke, D L 1970, corpus no 1520), and Kinghorn and Ashgrove, Methilhill in Fife (ibid, nos 1654 \& 1662). All would fall into the Late Style of Case's more simplified scheme (1977, 71-101). Although precise parallel features are lacking, the examples cited share the breakdown of these formal and decorative elements which characterize the earlier beaker tradition. On the basis of the main body of radiocarbon dates, a date range of $\mathrm{c} 1600 \mathrm{bc}$ to as late as $1450 \mathrm{bc}$ might be in keeping for the Strathallan vessel (Burgess 1980, 68). However, the date of $1000 \mathrm{bc} \pm 150$ (Q-764) for the Ashgrove grave mentioned above must be borne in mind, whatever the reservations about its validity.

Fragments of at least two vessels were recovered from the upper fills of two of the large post-pits of ring A. As in the case of the complete beaker (henge SF20), the fabric of the vessels represented by SF6-13 is coarse, and in places, especially on the base fragments, very loosely consolidated: there is certainly little comparison with the finer funerary beaker wares. The possible relationship of the henge sherds SF6, 9-12 and their tentative reconstruction as parts of a single vessel have already been discussed ( $\mathrm{p}$ 162): if correctly interpreted, the sherds and fragments suggest a vessel with some similarities to the complete beaker (SF20) discussed above, but the slightly more everted rim and more sophisticated decoration suggest a greater retention of traditional beaker traits. The 'bar chevron' decoration, if accurately reconstructed, is unusual for a Scottish beaker. Filled and contrast designs characterize Clarke's Southern British motif group $(1970,20,427)$ : however, as noted by Lanting and van der Waals, the naming of these groups is misleading $(1972,28-9)$, and many Scottish comb-decorated beakers incorporate filled triangles as elements of their decoration (Clarke, D L 1970, eg corpus nos 1622, 1645, 1708, 1710). Consequently, the elements of the pattern were already present in the 'grammar of ornament' of the Northern beaker series, at least from the conjectural Step 4 stage of Lanting and van der Waals $(1972,35)$. In one or two rare cases, the filled designs are even integrated to form the complete 'bar chevron', in the distinctive 'contrast' pattern, as on the beaker from Fingask, Perthshire (Clarke, D L 1970, corpus no 1741) or on the incomplete vessel from Gullane, East Lothian (ibid, corpus no 1620). The later Southern (S4) beaker from Balbirnie (Ritchie, J N G 1974, 18) similarly illustrates the adaptation of a related 'southern' motif to incised and jabbed techniques of decoration. A Late Style, or Step 6/7 position would be appropriate for the Strathallan sherds, with a tentative date range broadly similar to that suggested above for the complete beaker.

BRONZE AGE POTTERY

(i) Food vessels

A total of six food vessels is represented by sherds and complete pots recovered from the 
henge and barrow. All of the five which can be reconstructed are unexceptional examples of northern British food vessels falling into the categories of bipartite vase (henge SF17, 19; barrow SF42); ridged vase (barrow SF41); and ridged Irish-Scottish bowl (henge SF18), using the most recent terminology proposed by Burgess $(1980,86-9)$ whose reservations about such classification must be borne in mind.

The finest of the vessels is that recovered complete from burial B on the henge (SF17). The pot has been carefully made and ornamented in a form of zonal decoration consisting of horizontal grooves alternating with rows of jabbed impressions either applied singly or opposed to form simple bands of false-relief. Although the repertoire of techniques used may vary, broadly similar zonal arrangements of the decoration feature on many food vessels, but, in the absence of a full corpus, it is difficult to discern any detailed regional variations, although Pierpoint's survey certainly indicates their likely existence $(1980,87-9)$. In this connection, however, the Strathallan vessel assumes particular interest when compared with a food vessel (fig 29) excavated in 1905 from a cist at Cowdenhill, Bo'ness, West Lothian and now in the National Museum (NMAS: EE 114; Coles, F R 1906, 316-7; Callander 1924, 288-9). Although the Bo'ness vessel is slightly different in proportions and in aspects of its decorative scheme, these variations are outweighed by the points of similarity. The overall profile, the unusual stepped rim form, the method and overall arrangement of ornament, are all so close as to suggest manufacture of the Bo'ness and Strathallan vessels by the same hand or, at least, by potters familiar with each other's work (cf Pierpoint 1980, 119-21). An explanation for the occurrence of these two vessels in graves on sites some $36 \mathrm{~km}$ (c 23 miles) apart is not attempted here, but clearly (unless their similarity is dismissed as coincidence) the discovery has interesting implications for relationships between communities living either side of the Ochils in the Forth and Earn valleys. More detailed analysis is planned which, it is hoped, may throw further light on the presumed connection between the two vessels.

An incomplete vessel from the henge (SF19), represented by sherds recovered from burial $\mathrm{E}$, would have been of similar size and shape when complete but with a less regular zonal arrangement of jabbed, impressed cord and incised ornament. The remaining food vessel from the henge the squat ridged bowl, SF18 - is ornamented in an all-over design of vertical whipped cord maggot impressions. This technique of decoration is particularly widespread, although more typically applied in herringbone patterns or as elements of more complex decorative schemes.

Another typical use of maggot impressions is as a means of ornamenting the internal bevels of rims: such a usage occurs on the interior of one of the two substantially complete vessels from the barrow (SF41). The bone-end impressions which form the principal decoration represent another characteristic technique of the food vessel series, again originating in the impressed pottery styles of the late Neolithic (cf Longworth 1967, 74).

Bone-end impressions also ornament the incomplete vessel (SF42) recovered from the barrow, the sole example from the Strathallan sites of the classic 'Yorkshire Vase' category: the technique and arrangement of the decoration are somewhat atypical of this series, in which horizontal lines or chevron patterns of cord or incision tend to predominate, in places, it seems, virtually to the extent of orthodoxy. However, in assessing whether or not a particular vessel is 'typical' it is necessary to remember that the arrangement, and to a lesser extent, the technique of the decoration are the variables which allow the potter most scope for expression of individual taste, while still working the constraints of a particular technological tradition. The decorated base of this food vessel is also of interest: in view of the discovery that at least some food vessels may have contained a cereal-based liquid (or 'frumenty' - Hawkes 1977; 1981) is is tempting to suggest at least an occasional functional correlation: namely, that decoration was carried out, 
for much the same reason as on the bases of some traditional African beer pots today, to be seen by drinking companions when tipped up in use. In this respect it is interesting to note that, although rare, decorated bases occur particularly on handled beakers as well as on the occasional standard beaker (Clarke, D L 1970, 246; listed, 437). Decorated bases feature too on the products of the food vessel traditions - sporadically on the various vase types (eg Gibson 1978, cat no 43) but as a regular feature of the Irish-Scottish bowl series (Pierpoint 1980, 89).

Finally it is worth noting the small rimsherd (barrow SF25) identified as part of an unclassifiable food vessel on the basis of form and fabric, as this piece assumes some significance in view of its recovery from the infill of the central enclosure of the barrow.

The Strathallan food vessels may thus provide a representative group of the types of funerary food vessel current in the locality in the period c 1700-1400/1300 bc: relevant absolute dates are still at a premium and are usefully reinforced by the date of $1540 \mathrm{bc} \pm 65$ (GU-1381) for the skeletal remains associated with the food vessel (SF17) from burial B on the henge. The variety of forms and decorative schemes in the Strathallan group of vessels recalls Pierpoint's observation that his loosely defined 'Forth-Tay area' 'probably has a richer assortment of styles than any other area' $(1980,89)$, but this is probably no more than a reflection of the openness of this geographically central area of Scotland to diverse influences (Burgess 1980, 87).

\section{(ii) Cinerary urns}

Three cinerary urns recovered from the henge may represent the latest prehistoric pottery to be deposited on the site. Two were recovered, although stratigraphically unrelated, from the upper fill of a possible grave pit (F51). Certain features of the large collared urn, (SF21; burial H), including the internal moulding and its decoration, and the rim and collar forms, are sufficient to assign the vessel to Longworth's Primary Series (1961, 267-8), although the chronological validity of this typology has been undermined by recent excavations and the increasing availability of absolute dates (Burgess 1974, 180). The only formal feature of the vessel which calls for comment is the deep concave moulding on the interior of the rim, one of the more distinctive traits isolated by Longworth $(1961,266)$. Such internal mouldings are a very rare feature of Scottish collared urns, which typically have simple internally bevelled rims.

Whether or not the presence of these 'archaic' features really does place the Strathallan urn early in the Scottish collared urn series will remain uncertain in the absence of absolute dates and further excavation. Nevertheless it is worth remembering that the concept of 'trait loss' (as argued by Longworth 1961, 264-72) is generally accepted as a broad principle in the construction of, for example, beaker typologies.

The principal decoration of the vessel is also unusual: traces of a few random comb impressions, fingernail marks and fine incised hatched lines give a strong, though unverifiable, impression of initial experimentation by the potter with the idea of an infill design prior to the adoption (or retention) of a more open pattern of impressed cord lines. Although the principal decorative technique is common among the collared urn series, the rather irregular cord pattern is not one that can be readily matched, at least among the Scottish urns, where filled or panel motifs tend to predominate (Morrison 1968, 91). The collar of this vessel thus demonstrates that a variety of techniques coexisted and were potentially in the repertoire of the potter: that so many collared urns in fact possess recurrent features of form and decoration is surely a reflection of the strength of potting traditions, and of the importance of social constraints (such as religious conservatism) in limiting individual freedom of expression.

On the other hand, the next vessel discussed may possibly reflect one individual's taste or design preference. The undecorated urn (SF22), was found near the collared urn just discussed. 
The form of this vessel is unusual and it lacks close analogies among the northern urns. The presence of a deep moulding on the interior might suggest some relationship with the collared urn series in which this is a relatively more common feature than in other types, though, as noted above, still extremely rare among the Scottish vessels. Conversely, the overall profile of the vessel appears to have little in common with collared urns: if anything, the bipartite form and pronounced shoulder, marking a major, though weak, structural junction, have more in common with the manufacturing technique of enlarged food vessel urns. It is possible, in view of the presence of both urn types on the site, that some potter was attempting an unusual experiment, incorporating elements of more than one ceramic technological tradition. Further light is perhaps thrown on the vessel by its absence of decoration: though rare, undecorated collared urns do account for four of the 45 such vessels from SW Scotland recorded by Morrison $(1968,91)$ and occasional examples occur elsewhere (eg Loanhead of Daviot, Aberdeenshire (Kilbride-Jones 1936, 293, fig 7)) in contrast to all the known food vessel urns from northern Britain which without exception bear at least some ornament (Cowie 1978a, 24). Restricted decoration or an absence of ornament are characteristics of the cordoned and bucket urn types (Burgess 1980, 93) but the form of the Strathallan vessel clearly owes little to these.

Finally mention may be made of the food vessel urn found near the west entrance of the henge (SF23): the rim form, bipartite profile and size of the vessel and the technique and arrangement of the decoration are all characteristic of the type (Cowie 1978a, 13-31). Recent work has emphasized the relationship between these and the Bipartite Vases which form their counterpart among the smaller vessels of the food vessel repertoire (Burgess 1980, 91).

Food vessel urns are a common urn type in the region, and though the limited total numbers invite caution it is tempting to suggest that this reflects the adoption of the inurned cremation rite by local communities which remained predominantly food vessel users. It is difficult not to associate this change with the arrival of 'influences' accompanying the appearance locally of the collared urn tradition. However, the current limited range of radiocarbon dates allows only a very imprecise date range of $c 1600-1200 \mathrm{bc}$ for the currency of these types as funerary vessels (Burgess 1974, 178-82, 225-8).

\section{FABRIC ANALYSIS OF POTTERY FROM NORTH MAINS: SUMMARY OF THE RESULTS}

\section{Kenworthy}

All the sherds were examined under a binocular microscope at $\times 20$ magnification. Twelve different fabrics were established on the basis of variations noted in the general texture, the appearance of the clay matrix and the nature, size and quantity of the inclusions. Fabrics A, B, C, D, E and F occur (with one possible exception) only in the pottery from the barrow, fabrics $H, I, J, K$ and $L$ only in that from the henge, the fabric $G$ presenting the only overlap between the two sites.

All the fabrics except $\mathrm{H}$ and $\mathrm{L}$ contain fragments of various rocks, notably schists (including a quartz-biotite schist), granite, at least one type of basalt and another unidentified fine-grained igneous rock; two or more of these frequently occur in the same fabric. All the fabrics also contain various individual minerals in varying amounts and combinations; these comprise mainly quartz, feldspar, muscovite and biotite mica, and possibly other ferromagnesian minerals.

It cannot be definitely demonstrated that the pottery was not deliberately gritted, but the occurrence of two or more types of rock in the same fabric suggests that it is much more likely that the rock fragments were present in the clay from which the pottery was manufactured, and that the individual minerals result from the breakdown, either by natural weathering processes within the clay source or by the working of the clay in the process of pottery manufacture, of the rock fragments into their individual constituents. 
The sources of the clay used for the different fabrics cannot be positively identified, but there is a strong probability that all the fabrics are of local manufacture. The local boulder clay of the glacial drift, overlying old red sandstone, would be liable to contain pebbles of several types of rock, including all these identified in the pottery. The fact that the pottery is not all homogeneous and that 12 separate fabrics can be recognized would indicate the use at different times and on different occasions of different parts of the boulder-clay deposit, which would be expected to show minor geographical and stratigraphical variations in its composition; this would account too for the very small degree of overlap of fabric between the barrow and the henge.

The two fabrics, $\mathrm{H}$ and $\mathrm{L}$, which contain no rock fragments, may also be essentially the same as the others; they could derive from pockets of slightly finer, more weathered clay, or they may have been more thoroughly worked by the potter, reducing any rock fragments to their constituent minerals. Fabric $\mathrm{L}$ is in any case very soft, and the 'sherds' of this fabric are probably pieces of clay daub, which in itself suggests a local origin, as raw clay for this purpose is unlikely to have been imported from any distant source.

\section{THE USE OF STONE AT NORTH MAINS: GENERAL DISCUSSION}

\section{R Wickham-Jones}

In this discussion reference will be made primarily to the assemblages from the henge and barrow. The ring-ditches produced a very small assemblage which, while not particularly diagnostic, fits quite easily into the stone technology seen upon the other two sites. This small assemblage must, however, reflect a different use of stone upon the ring-ditches that did not lead to the deposition of any number of pieces. The collections from both the henge and barrow sites may be divided into two main categories, those pieces that were associated with burials and those pieces from elsewhere. Those pieces included within the burials demonstrate that upon both sites stone played some part in the burial ritual but it is impossible to infer any greater detail. From a comparison of all the assemblages, however, more information may be drawn and it is possible to see that the use of stone upon both sites was very similar.

Comparable raw materials were used upon each site. The main material used was large, good quality nodules of pebble flint supplemented by some pitchstone, a little quartz and, in the henge, agate, while upon the barrow mudstone, probably from a broken polished axe, was flaked and re-used. It is possible that slightly larger, better quality pebbles of flint are represented in the barrow assemblage but this may be a reflection of the larger tools that made up the collection because of the different tasks involved, rather than of a difference in material and source.

Neither assemblage contains much evidence of on-site knapping but from the detail that does exist it is apparent that very similar technologies were practised upon the two sites. The nodules were flaked with a variety of hammers using direct percussion upon artificial, sometimes facetted, platforms. On both sites this resulted in an assemblage of regularly shaped flakes, some of which were selected, upon a basis of quality and size, for retouching work. The morphology of the tools so produced differs on each site, only on the barrow are recurring types found, but both sites reflect the use of different types of retouch to alter the shape of a flake and to create a particular edge.

Despite the lack of recurring morphological types the basic nature of each assemblage is very similar as each reflects the use, subsequent alteration and loss of prefabricated tools with no evidence of their manufacture. Just as the two sites are themselves different, so the actual tools used differ but the processes behind the formation of the two assemblages are comparable.

It is possible that, seemingly in contrast to the barrow, the henge monument was kept clean, thus artificially diminishing the size of the assemblage, but enough has remained to demonstrate this general similarity of formation and composition. Although the actual tasks involved may 
differ, the use of stone upon the two sites is almost identical. Each assemblage is largely determined by the raw material and technology used as well as by the tasks involved but in neither cases does culture appear to have been a prime determinant in the formation of the collection.

\title{
ANALYSIS OF PITCHSTONE FROM THE HENGE AND BARROW AT NORTH MAINS
}

\section{O W Thorpe and R S Thorpe, Department of Earth Sciences, The Open University}

Four pitchstone pieces were submitted for neutron activation analysis; two from each site (Henge SF86 and 93; Barrow SF69 and 71). One from each site was also thin sectioned (Henge SF86; Barrow SF69. These were compared with analyses of samples from pitchstone sources on Arran, Mull, Ardnamurchan and Eigg. All four of the pieces are certainly of Arran pitchstone. The examination of the thin sections suggests that both pieces originated in the Clauchland Hills area of Arran, at a sill-like outcrop of aphyric pitchstone, in the E part of the island, south of Brodick, also known as the Corriegills pitchstone (NGR NS 041 341). See also Thorpe and Thorpe 1984.

\section{RADIOCARBON DATES FROM THE NORTH MAINS SITES}

\author{
M Stenhouse, Department of Chemistry, University of Glasgow
}

\section{COMMENT ON NORTH MAINS BARROW SERIES}

Samples GU-1102, -1103, GU-1134 to -1136

Although the errors quoted are as summation of all quantifiable errors associated with the dating process, the contribution from the statistical uncertainty of activity measurements is by far the dominant term. In this respect, the high error for sample GU-1135 reflects the small amount of carbon $(<1 \mathrm{~g})$ analysed. For such small samples, then, the possibility of non-quantifiable errors, eg the effect of contamination by intrusion, should be carefully considered as potential sources of additional uncertainty.

TABle 9

Radiocarbon dates

\begin{tabular}{|c|c|c|c|c|}
\hline Laboratory No & Period & Feature & Date (uncorrected) & Association \\
\hline \multicolumn{5}{|l|}{ Henge } \\
\hline GU-1350 & IV & F6 & $905 b c \pm 85$ & \\
\hline GU-1351 & IV & F6 & $1085 b c \pm 70$ & \\
\hline GU-1352 & II (late) & $\mathrm{A} / 7$ & $2330 \mathrm{bc} \pm 60$ & \\
\hline GU-1353 & II (primary) & $\mathrm{A} / 7$ & $2155 b c \pm 60$ & \\
\hline GU-1354 & II (primary) & $\mathrm{A} / 5$ & $2090 \mathrm{bc} \pm 70$ & \\
\hline GU-1381 & III & Burial B & $1540^{\circ} b c \pm 65$ & Food vessel \\
\hline GU-1382 & V & LG3 & ad $760 \pm 60$ & \\
\hline GU-1435 & II (late) & $\mathrm{A} / 1$ & $2065 b c \pm 65$ & \\
\hline GU-1436 & II (late) & $\mathrm{A} / 13$ & $2180 b c \pm 60$ & \\
\hline GU-1437 & IV & F5 & $895 b c \pm 60$ & \\
\hline GU-1438 & III & F33 & $1450 b c \pm 60$ & $\begin{array}{l}\text { Barbed and tanged } \\
\text { point }\end{array}$ \\
\hline \multicolumn{5}{|l|}{ Barrow } \\
\hline GU-1102 & III C/D & & $1665 b c \pm 85$ & \\
\hline GU-1103 & IIIB & & $1785 b c \pm 85$ & \\
\hline $\begin{array}{l}\text { GU-1134 } \\
\text { ((GU-1135) } \\
((\text { GU-1136 }\end{array}$ & I/II/IIIA-D & & $\begin{array}{c}1855 \text { bc } \pm 100 \\
\text { ad } 490 \pm 220) \\
100 \text { years) }\end{array}$ & \\
\hline \multicolumn{5}{|l|}{ Ring-ditch area } \\
\hline GU-1546 & $\begin{array}{l}\text { Predates } \\
\quad \text { ring-ditch } 2\end{array}$ & F3 & $2690 b c \pm 65$ & $\begin{array}{l}\text { 'Western Neolithic' } \\
\text { pottery }\end{array}$ \\
\hline
\end{tabular}




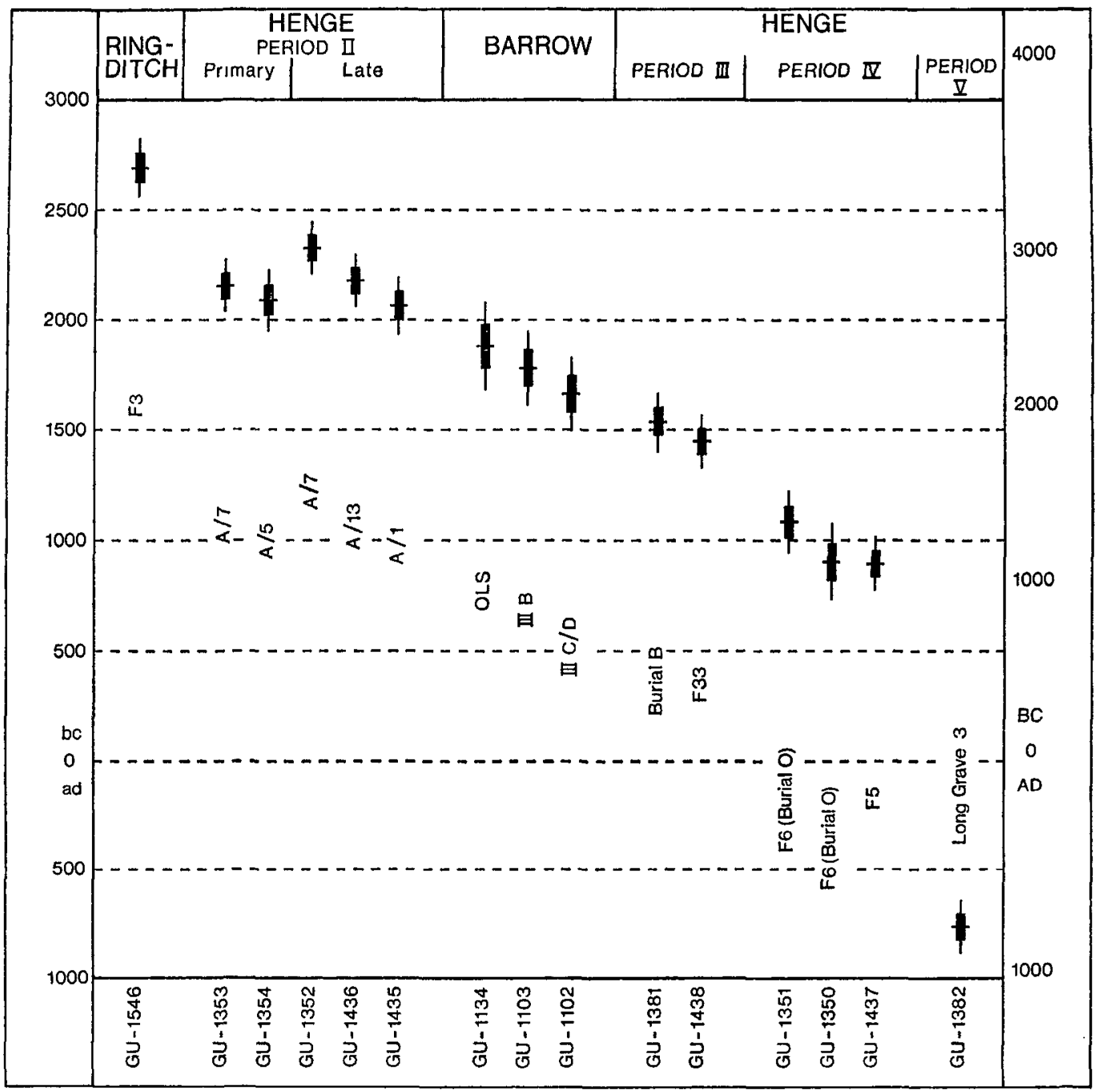

Fig 65 Radiocarbon dates (expressed at one and two sigma levels: calibration according to Clark 1975)

\section{AN ACCOUNT OF THE SOILS AT NORTH MAINS}

\section{J C C Romans and L Robertson, Macaulay Institute for Soil Research, Aberdeen}

\section{INTRODUCTION AND SITE DESCRIPTION}

The barrow and henge at North Mains, Strathallan were sited on a level gravel terrace at c $30 \mathrm{~m}$ OD between the River Earn and the Machany Water, c $1 \mathrm{~km}$ upstream from their junction near Kinkell Bridge. The terrace gravels vary in depth from c $0.5 \mathrm{~m}$ (over solid rock), in a soil profile pit dug in the field $\mathrm{N}$ of the mound, to more than $2.5 \mathrm{~m}$ in the ditches of the henge. In the peripheral ditch around the mound, compact laminated fluvioglacial silt was present below the gravel on the W, $\mathrm{S}$ and $\mathrm{E}$ sides, coming within $1 \mathrm{~m}$ of the surface on the $\mathrm{E}$ side. Laminated silt was not seen below the gravels in either the ditch sections or postholes on the henge site, though contorted lumps of silt were sometimes present within the gravels as included erratics. Comparison of the amounts of silt and clay in laminated silt from a profile below the mound, with the amounts present in similar material from soil survey profile pits at Denfield 
(NN 948 172) and Mill of Gask (NN 977 176) showed that there was a fall in silt content and a rise in clay content from $\mathrm{W}$ to $\mathrm{E}$. This suggests that the laminated silts had settled out from the meltwaters of a small ice lobe retreating into Glen Turret and that the overlying gravels were subsequently deposited after a minor glacial advance. The presence of a silty fine sandy loam topsoil above the gravel suggests some later deposition of wind-blown loess.

The impressive height and volume of the mound appeared to afford considerable protection to any buried land surface which might lie beneath it. On closer examination, however, it was evident that the greater part of the mound on the $\mathrm{W}$ and $\mathrm{N}$ sides was composed of stony, coarse-textured material which had been penetrated and disturbed by rabbit burrows and mature tree roots. Attention was therefore concentrated on the SE quadrant of the excavation where the upper $2 \mathrm{~m}$ of the highest part of the mound consisted of a coherent deposit of reddish brown silty fine sandy loam. There was a stony surface layer with scattered patches of stones and boulders below. Sublinear horizontal markings, which were most clearly seen in the lower part, suggested that this layer consisted of surface turf skimmings. Below this reddish brown turf layer there was up to $1.5 \mathrm{~m}$ of very compacted olive, yellowish brown and brown silt. The silt was in turn underlain by large boulders and a stony rather coarse textured reddish brown deposit which appeared to be derived from a mixture of surface soil and gravelly subsoil lying upon the prehistoric land surface. Even in the SE quadrant the roots of c 150 year old beech and oak trees had grown right through the mound, locally penetrating the old land surface below.

As first exposed, the buried soil below the SE quadrant had a very uniform firm non-porous dark reddish grey (5YR4/2) silty clay loam surface layer c $3 \mathrm{~cm}$ thick with weak coarse prismatic structure. Immediately below there was a red $(2.5 \mathrm{YR} 4 / 8)$ soft thin iron pan which varied from a continuous slightly irregular ochreous line to discontinuous patches of ochreous mottling. The surface $A_{2} g$ horizon and thin iron pan were underlain by a dark reddish-brown (5YR3/4) silty fine sandy loam A horizon with medium subangular blocky structure. The $\mathrm{A}$ horizon was somewhat softer and more friable than the $\mathrm{A}_{2} \mathrm{~g}$ horizon and was up to $14 \mathrm{~cm}$ thick where recorded. Below the A horizon there was a yellowish red (5YR4/6) silty fine sandy loam $B$ horizon with weak medium subangular blocky structure, soft and friable, and c $17 \mathrm{~cm}$ thick. The A and B horizons were moderately stony, with small stones not generally larger than 3 or $4 \mathrm{~cm}$ in the A, and ranging from 1 to $12 \mathrm{~cm}$ in the B horizon. Below the B horizon there was a fairly rapid transition into dark reddish brown (5YR4/4 to $3 / 4$ above, becoming $5 Y R 3 / 3$ to $3 / 4$ below) gravel in which fairly small flat tabular stones tended to predominate. The upper $14 \mathrm{~cm}$ of the gravel contained a fair amount of interstitial fine sandy loam and the gravel deposit was generally compact down to $\mathrm{c} 75 \mathrm{~cm}$ below the old land surface. The lower part of the gravel was loose and incoherent with more angular and subrounded stones. It was separated from the compact upper part by a 10-12 cm thick band of dark reddish brown (5YR3/3) loamy coarse sand. About $170 \mathrm{~cm}$ below the old land surface there was a sharp change into brown (7.5YR5/4) and reddish brown (5YR5/4) finely laminated silt, with brown (10YR5/3) laminated silt below. The depth of this fluvioglacial silt deposit was not investigated as there was a considerable inflow of water from the basal part of the gravel.

An interesting feature of the buried land surface below the mound was the presence of parallel E-W trending low cultivation ridges spaced c $2 \mathrm{~m}$ apart from crest to crest. Cultivation ridges appeared to be absent from the area of the central enclosure. The $A_{2} g$ horizon with its underlying soft thin iron pan ran ubiquitously over ridges, furrows and the flatter ground in the central area. This indicated that $A_{2} g$ formation post-dated the last period of cultivation before the mound was constructed. Near-surface formation of a thin iron pan has been associated elsewhere (Crompton 1966) with the development of a mor humus mat on deteriorating acid pasture under rather wet climatic conditions. However, there is no evidence of surface mor humus in any of the buried soil profiles examined or on any of the skimmed turf used to build the mound, and $\mathrm{C} / \mathrm{N}$ ratios are uniformly low (tables $11,12,14$ ) so that this interpretation seems inappropriate at Strathallan. The compacted appearance of the surface of the $\mathbf{A}_{2} \mathrm{~g}$ when it was freshly exposed looked very like the puddled and compacted surface left on a present day arable field after sheep have been put in to graze off a turnip crop. A similar surface puddling effect was noted in January 1969 on a newly drained grass ley, only a few kilometres from Strathallan, after it had been grazed by sheep in wet conditions. The surface few centimetres became gleyed with some development of scattered ochreous mottling below (BM Shipley, pers comm). The rainfall at Strathallan in January 1969 was $97 \mathrm{~mm}$, a figure close to the $1969-79$ average of $92 \mathrm{~mm}$. There were no cultivation ridges below the henge bank, but a similar soil profile with gleyed surface $\mathbf{A}_{2}$ g horizon, this time without underlying soft thin iron pan, was present. In general terms grazing seems to provide a very reasonable explanation for the development of a compacted $\mathrm{A}_{2} \mathrm{~g}$ horizon with, or without, an underlying soft thin iron pan. Total 
phosphate levels of around $200 \mathrm{mg} \mathrm{P}_{2} \mathrm{O}_{5}$ per $100 \mathrm{~g}$ soil in the buried land surface below the mound and around $90 \mathrm{mg} \mathrm{P}_{2} \mathrm{O}_{5}$ per $100 \mathrm{~g}$ soil in the land surface below the henge suggest that stocking was more intensive on the mound site than on the henge site. Whether this might reflect protective night folding of stock on one site, as opposed to seasonal grazing of arable land on the other, must remain conjectural.

Examination of the residual baulk sections left after excavation indicated that the lowest layers of the mound did not contain any material which could not have been quarried from the peripheral ditch. By contrast the excavation spoil dumps contained many substantial glacial erratics. The largest erratic boulders were in the 40-60 cm size range, and the many smaller stones were all at least two or three times larger than the coarsest gravel pebbles seen in the peripheral ditch sections or in soil profile pits. Many of these stones and boulders had subaerially weathered rims. A large slab of conglomerate which had been used as the capstone of cist burial B, on the henge site, had smooth rounded corners on one side of the slab and sharp edges on the other, indicating that it had formerly lain, with one surface buried and the other exposed to subaerial weathering, for a long period before it was used as a capstone. No large stones were present on any part of the buried agricultural land surface exposed by excavation. It may therefore be inferred that the land surface was cleared of stones long before the mound was constructed, and that many of the local clearance cairns were used up in the construction of the mound, perhaps making available some additional cleared ground to offset that lost below the mound.

\section{SOIL SAMPLING}

During the excavations 19 soil samples were collected for analysis from the mound site. These samples came from the post-construction soil profile exposed on the S-facing side of the E baulk (table 10) and from a profile dug into the buried land surface below the SE quadrant (tables 11, 12). A sample of the present day surface soil was also taken just beyond the peripheral ditch outside the SE quadrant (table 13).

Undisturbed vertically oriented soil samples for micromorphological examination were collected in standard 75 by 55 by $45 \mathrm{~mm}$ aluminium frame boxes, or in monolith boxes (which were later subsampled) from the $S$ face of the. E baulk, from the buried soil below the SE quadrant, from the buried soil below the NE quadrant, from the central enclosure above and below the old land surface, and from material infilling the ditch on the $\mathrm{N}$ side of the mound. Further undisturbed samples were taken from the buried soil surface below the henge bank on the S and NW sides. Ninety soil thin sections, mostly c 75 by $55 \mathrm{~mm}$ were subsequently prepared after the soil samples had been freeze-dried and impregnated with Bakelite 17449 polyester resin.

\section{MICROMORPHOLOGY}

Because of the very large number of soil sections, their detailed examination had to be restricted to those micromorphological features likely to elucidate the stage of soil profile development, palaeo-landuse, or mound construction procedure. Special attention was given to two features in the thin sections. First, to the presence or absence of iron-stained weathering rims on small stones, and the intensity of any secondary peripheral bleaching of such iron-stained rims; and secondly, to the total number of soil pores within each thin section which were lined or infilled with coatings of oriented clay, with a qualitative assessment of the relative thickness of pore linings.

Previous work on soils of the Ettrick Association (Romans \& Robertson 1975) has shown that, where suitable rock material is present in the soil, the presence or absence in the upper $30 \mathrm{~cm}$ of the profile of iron-stained weathering rims on small stones and the intensity of any secondary peripheral bleaching of such iron-stained rims can be correlated with profile macromorphology. The A horizons of brown forest soils which are, or have until recently been, under more or less continuous deciduous woodland, may or may not contain small stones with weakly developed iron-stained rims. Such soils usually still support an earthworm population. When the forest cycle has been severely disrupted, acid brown soils are developed, and $\mathrm{A}$ horizon thin sections may show a range of stone rim weathering from well defined iron-stained rims to iron-stained rims with peripheral bleaching $0.1 \mathrm{~mm}$ or rather more in thickness. Earthworm activity in these soils is minimal. When the bleached rims on stones exceed $1 \mathrm{~mm}$ in thickness near the surface and decrease gradually with depth, fading out not more than $25-30 \mathrm{~cm}$ below mineral ground surface, the semi-natural soil is usually a podzol or peaty podzol. Earthworms are absent in this context.

At Strathallan the presence of scattered small stones with iron-stained rims, on which secondary bleached rims up to $0.1 \mathrm{~mm}$ thick are occasionally present, is a common feature of all the thin sections 
TABle 10

Strathallan Mound - post-construction soil profile sampled on $\mathrm{S}$ face of $\mathrm{E}$ baulk

$\begin{array}{cccc}\text { Horizon } & \begin{array}{c}\text { Depth } \\ (\mathrm{cm})\end{array} & \begin{array}{c}\% \text { USDA } \% \text { USDA } \\ \text { sand }\end{array} & \begin{array}{c}\text { silt } \\ \text { A }\end{array} \\ 0-5 & 36 & 32 \\ \text { A } & 5-10 & 58 & 35 \\ \text { A } & 10-15 & 44 & 32 \\ \text { A } & 15-20 & 49 & 35 \\ \text { B/C } & 58-67 & 36 & 37 \\ \text { C } & 150-156 & 35 & 34 \\ \text { C/D } & 250-260 & 23 & 59\end{array}$

$\begin{array}{rr}\begin{array}{r}\% \\ \text { clay }\end{array} & \begin{array}{r}\text { pH } \\ \mathrm{H}_{2} \mathrm{O}\end{array} \\ 18 & 4 \cdot 5 \\ 7 & 4 \cdot 3 \\ 12 & 4 \cdot 4 \\ 16 & 4 \cdot 4 \\ 27 & 4 \cdot 0 \\ 31 & 4 \cdot 3 \\ 18 & 5 \cdot 2\end{array}$

$\% \mathrm{C}$
$9 \cdot 16$
$11 \cdot 50$
$9 \cdot 60$
$7 \cdot 28$
$0 \cdot 77$
$0 \cdot 72$
$0 \cdot 07$

\section{$\% \mathbf{N}$}

$0 \cdot 62$

0.85

0.66

0.46

0.08

0.09

0.04

$* \% \mathrm{OM}=1 \cdot 72 \times \% \mathrm{C}$

$\dagger$ EDTA = Ethylenediaminetetra-acetic acid

$\ddagger$ ND= Not determined
TABLE 11

Strathallan mound - buried soil profile below SE quadrant (sampled 1978)

$\begin{array}{cccc}\text { Horizon } & \begin{array}{c}\text { Depth } \\ (\mathrm{mm})\end{array} & \begin{array}{c}\% \text { USDA } \\ \text { sand }\end{array} & \begin{array}{c}\text { USDA } \\ \text { silt }\end{array} \\ \text { Aol }_{1} & 0-3 & 50 & 30 \\ \text { A }_{2} \mathrm{~g} & 0-30 & 45 & 30 \\ \text { A } & 30-90 & 43 & 33 \\ \text { C } & 510-590 & 79 & 14\end{array}$

$\%$
Clay
20
25
24
7

$\mathrm{pH}$
$\mathrm{H}_{2} \mathrm{O}$
$5 \cdot 7$
$5 \cdot 7$
$5 \cdot 7$
$5 \cdot 4$

\section{$\% \mathrm{C}$}

$$
0.43
$$$$
0.55
$$$$
\begin{aligned}
& 0 \cdot 55 \\
& 0 \cdot 26
\end{aligned}
$$

$0 \cdot 15$

$\% \mathrm{~N}$

0.05

0.08

0.06

0.03

C/N
$8 \cdot 6$
$6 \cdot 9$
$4 \cdot 3$
$5 \cdot 0$

$\mathrm{C} / \mathrm{N}$
$8 \cdot 6$
$6 \cdot 9$
$4 \cdot 3$
$5 \cdot 0$

$\% \mathrm{OM}^{*}$

$\mathrm{mg} \mathrm{P}_{2} \mathrm{O}_{5}$

$0 \cdot 74$

0.94

0.45

0.27

soil

extracted by

212 NDt NDt

188

205

250

240
22

133

186

22

TABLE 12

Strathallan mound - buried soil profile below SE quadrant (resampled 1979)

$\begin{array}{lccc}\text { Horizon } & \begin{array}{c}\text { Depth } \\ \text { (cm) }\end{array} & \begin{array}{c}\% \text { USDA } \\ \text { sand }\end{array} & \begin{array}{c}\% \text { USDA } \\ \text { silt }\end{array} \\ \text { A2g } & 0-3 & 39 & 36 \\ \text { A } & 10-14 & 40 & 42 \\ \text { B } & 17-22 & 42 & 46 \\ \text { C } & 71-76 & 85 & 9 \\ \text { C } & 76-86 & 96 & <1 \\ \text { C/D } & 169-176 & 14 & 76 \\ \text { C/D } & 179-185 & 18 & 74\end{array}$

$\begin{array}{rcc}\begin{array}{r}\% \\ \text { clay }\end{array} & \mathrm{pH} & \\ 25 & \mathrm{H}_{2} \mathrm{O} & \% \mathrm{C} \\ 18 & 5 \cdot 7 & 0 \cdot 39 \\ 12 & 5 \cdot 6 & 0.58 \\ 6 & 5 \cdot 5 & 0.37 \\ 4 & 6 \cdot 0 & 0 \cdot 13 \\ 10 & 6 \cdot 3 & 0.06 \\ 8 & 5 \cdot 7 & 0.11 \\ & 5 \cdot 9 & 0.12\end{array}$

$\% N$
0.07
0.07
0.08
0.11
0.04
0.06
0.08

$\mathrm{mg} \mathrm{P}_{2} \mathrm{O}_{5}$ per $100 \mathrm{~g}$

ppm Fe ppm Al extracted by

$\mathrm{C} / \mathrm{N}$
$5 \cdot 6$
$8 \cdot 3$
$4 \cdot 6$
$1 \cdot 2$
$1 \cdot 5$
$1 \cdot 8$
$1 \cdot 5$

$\% \mathrm{OM}^{*}$
$0 \cdot 7$
$1 \cdot 0$
$0 \cdot 6$
$0 \cdot 2$
$0 \cdot 1$
0.2
0.2

soil
194
225
249
116
192
235
220

$\begin{array}{rr}0.05 \mathrm{~m} & \text { EDTA } \dagger \\ 260 & 118 \\ 150 & 133 \\ 61 & 131 \\ 37 & 44 \\ 17 & 43 \\ 55 & 18 \\ 64 & 17\end{array}$


TABLE 13

Modern ploughed soil, sampled near Strathallan Mound

$\begin{array}{lc} & \% \mathrm{OM}^{*} \\ \text { Surface soil sample from outside ditch, SE quadrant, Strathallan Mound } & 9 \cdot 8 \\ \text { Surface soil sample from soil profile N Mains of Strathallan NN 925/165 } & 8 \cdot 4\end{array}$

TABLE 14

Strathallan henge - buried soil below NW bank

$\begin{array}{ccccccc}\text { Horizon } & \begin{array}{c}\text { Depth } \\ (\mathrm{mm})\end{array} & \% \mathrm{C} & \% \mathrm{~N} & \mathrm{C} / \mathrm{N} & \% \mathrm{OM}^{*} & \begin{array}{c}\text { mg } \mathrm{P}_{2} \mathrm{O}_{5} \\ \text { soil }\end{array} \\ \mathrm{A} & \mathbf{0 - 1 5} & \mathbf{0 . 4 9} & 0.06 & 8.2 & 0.8 & 92 \\ \mathrm{~A} & 15-30 & 0.56 & 0.06 & 9.3 & 1.0 & 84 \\ \text { A } & 60-75 & 0.49 & 0.05 & 9.8 & 0.8 & 86\end{array}$

prepared from palaeoturf material from the upper $2 \mathrm{~m}$ of the mound and from the surface layers of the buried soils under both the mound and the henge bank. They were also present in the surface layers of the present day profile developed on the mound. This indicates that acid brown soils with very similar morphology and minimal earthworm population were widely distributed over the flat terrace when the henge and mound were constructed. Clearly also the contemporary soil profile developed on the mound has retained the morphological characteristics of an acid brown soil: though the higher carbon/nitrogen ratios and the pattern of EDTA extractable iron and aluminium values (table 10) indicate enhanced leaching and a tendency towards podzolization in the surface layers.

The micromorphological indications of a low level worm population are confirmed by the low $\mathrm{pH}$ (4.1-4.3) and low organic matter content (c $1 \%$ with carbon/nitrogen ratio 7:9) of turf material from the mound (Edwards \& Lofty 1972; 1974). The somewhat higher $\mathrm{pH}(5 \cdot 7)$ recorded in the A horizon of the buried soil under the mound probably reflects recent agricultural liming, a porous gravel subsoil, and postconstruction fluctuations of the soil water table below the mound.

The numbers of oriented clay-lined soil pores present in all soil thin sections have been counted using a Zeiss traversing microscope, and the relative thicknesses of the pore linings have been evaluated on a qualitative basis using a low power stereomicroscope. Thin sections covering the upper $230 \mathrm{~cm}$ of the $S$ face of the $E$ baulk were available. This included $\mathrm{c} 200 \mathrm{~cm}$ of reddish brown turf material and $30 \mathrm{~cm}$ of the underlying compact olive and brown silt. Three additional thin sections were spaced out over the lower part of the compact silt down to $\mathrm{c} 350 \mathrm{~cm}$. The general distribution of oriented clay-lined pores throughout the upper $230 \mathrm{~cm}$ of the mound is shown in fig 66. Two major pore count maxima are present. Individual pores are most heavily lined with oriented clay at the maxima, and most weakly lined at the minimum (c $180 \mathrm{~cm}$ ). In most soil pores the clay lining is thickest on the lower wall of the pore, as would be consistent with vertical translocation of a colloidal suspension of clay.

At the top of the mound the $0-100 \mathrm{~mm}$ and 100-255 mm layers form the upper and lower parts of an A horizon with loose, fine, crumb structure. In the $0-76 \mathrm{~mm}$ soil thin section there is a very loose open fabric consisting of pellety mineral debris, organic litter and tree roots. In the lower part a few small darkly stained remnants of palaeoturf material are present, one with part of an oriented clay pore lining still present. In thin sections 2 and $3(100-176 \mathrm{~mm}$ and $177-253 \mathrm{~mm})$ both loose pellety mineral debris and unstained fairly coherent fragments of palaeoturf are present; some of these palaeoturf fragments are up to $15 \mathrm{~mm}$ in size near the base of thin section 3. Some oriented clay-lined pores are present in the palaeoturf fragments. The B horizon of the modern mound profile with medium subangular blocky structure was recorded between 255 and $420 \mathrm{~mm}$. In thin section $4(255-332 \mathrm{~mm})$ coherent peds of palaeoturf begin to dominate the fabric and the count of oriented clay-lined pores rises to 27 . Thin section $5(335-411 \mathrm{~mm})$ is predominantly palaeoturf material, locally broken up by modern tree roots, and the count of oriented clay-lined pores rises sharply to c 560. From thin section $6(425-501 \mathrm{~mm})$ to thin section $26(2011-2065$ $\mathrm{mm}$ ) the palaeoturf material is relatively unmodified except by the local penetration of modern tree roots. The highest counts of oriented clay-lined pores were recorded in thin section 7 (1027 pores) at $520-597 \mathrm{~mm}$ and in thin section 11 (1186 pores) at $834-902 \mathrm{~mm}$. Two extra soil thin sections, A and B on fig 66, were taken c $2 \mathrm{~m} \mathrm{~W}$ of the main sampling line. A (540-616 mm below surface) came from the charcoal-lined 
base of a shallow concave feature at the top of the mound, and B $(616-692 \mathrm{~mm})$ from consolidated palaeoturf material immediately below this feature. The actual numbers of oriented clay-lined pores in soil thin sections A and B are somewhat greater than in corresponding thin sections from the mound profile, but the distribution pattern of the pores and the qualitative thickness of pore linings closely match the main profile. In thin section $A$ there is a prominent black charcoal band of variable thickness between 57 and $58 \mathrm{~cm}$ and more scattered charcoal can be readily seen without a hand lens, both above and below the main band. Below the charcoal band there is a $1 \mathrm{~cm}$ thick grey band with an iron-stained base. The lower part of slide A appears to be palaeoturf, quite comparable with the rest of the upper $2 \mathrm{~m}$ of the mound. Thin section B again consists of normal palaeoturf, but the amount of scattered charcoal present for $10 \mathrm{~cm}$ below the charcoal band (in thin section A) is significantly higher than in the palaeoturf slides from the main profile. Oriented clay is frequently seen to infill large pores in charcoal, or to be attached to charcoal fragments, throughout thin sections $\mathbf{A}$ and $\mathbf{B}$. This would seem to suggest that a fire was lit in a hollow at the top of the mound, before the mound had consolidated enough to prevent pieces of charcoal falling down cracks and before the oriented clay, now infilling soil pores, was mobilized and translocated through the mound.

Within the turf material forming the upper $2 \mathrm{~m}$ of the $\mathrm{E}$ baulk the count of oriented clay-lined pores per thin section ranged from several hundred to over a thousand. The consistent vertical alignment of the great majority of these pores suggests that they must have been lined with translocated clay after the mound was constructed. In thin section 11 fragments of thin iron pan were penetrated by unstained oriented clay, supporting the same conclusion. In thin section 17 there were two sets of oriented clay-lined pores aligned approximately at right angles to each other, as might be expected if the weaker set was present in the turf as cut from the palaeo-land surface, and the vertically aligned dominant set was developed after the palaeoturf was incorporated into the mound. The upper pore count maximum extended from thin section 7 to thin section 11 .

Both the numbers of oriented clay-lined pores and the thickness of the pore linings declined gradually, if somewhat irregularly, below thin section 11 . In thin section $23(1744-1815 \mathrm{~mm})$ oriented clay-lined pores were minimal in numbers and very weakly lined. In thin section $24(1845-1920 \mathrm{~mm})$ there was a narrow band of well defined oriented clay-lined pores. Lower down both the number of pores and the thickness of pore linings increased to a second maximum in thin sections 25 (1925-2002 $\mathrm{mm}$ ) and 26 and in the upper half of thin section 27 (2069-2124 mm). In thin section 27 pores very solidly infilled with oriented clay were present at the interface between reddish brown turf material and the olive and brown compacted silt below. A little of the translocated clay penetrated the interface and was seen to be lining soil pores in fragmented silt which infilled the interped spaces between relatively undisturbed lumps of laminated silt; again confirming that considerable mobilization and translocation of clay took place after the construction of the mound. The compact silt deposit extended downwards from c $208 \mathrm{~cm}$ to $\mathrm{c} 350 \mathrm{~cm}$ at the chosen line of sampling on the $\mathrm{E}$ baulk. Thin sections 27, 28, 29 and 30 gave more or less continuous cover from c $2080 \mathrm{~mm}$ to $2305 \mathrm{~mm}$, with the additional thin sections 32, 33 and 34 spaced out below. The presence of occasional mechanically deformed oriented clay-lined pores within slightly distorted but otherwise undisturbed lumps of laminated silt throughout these sections indicates that there had been some translocation of clay in the prehistoric soil profile before the mound was built, and that this material probably came from the lower half of the peripheral ditch around the SE quadrant.

In thin section 27 there was quite a strong iron-manganese pan just above the interface between turf and silt with some local penetration into the silt. Pores lined with oriented clay at and just above the interface were iron-stained, indicating that this pan had formed after the deposition of the oriented clay. This deposition of iron and manganese appears to reflect post-construction gleying above the turf/silt interface after its permeability had been reduced by an initial downwash of clay.

After the mound had been removed, samples were taken from the prehistoric land surface. In the NE quadrant one of the prehistoric cultivation ridges constructed from normal reddish brown surface soil was sampled to $286 \mathrm{~mm}$, and another made of darker soil (present as scattered local patches, considered by the excavator to represent former tree-stump locations) was sampled to $46 \mathrm{~cm}$. Two single $75 \mathrm{~mm}$ samples were taken from the flatter ground in the central enclosure, one near the supposed entrance and the other near the timber wall. The deep profile cut into one of the cultivation ridges in the SE quadrant (previously described above) was sampled more thoroughly. Six samples covered the upper $485 \mathrm{~mm}$ of the buried profile with a seventh at 545-616 mm. Ten more samples were taken at irregular intervals between 72 and $192 \mathrm{~cm}$ below the old land surface, wherever sampling was practicable within the gravel and in the upper layers of the laminated silt below the gravel. 
There were no prehistoric cultivation ridges below the henge bank, and the buried land surface was sampled to $255 \mathrm{~mm}$ on the S side and to $13 \mathrm{~cm}$ on the NW side.

Soil pores with linings of oriented clay sufficiently substantial to be seen under a low power stereomicroscope were present in all the buried soils below the mound and henge, but were very scarce in the two samples from the central enclosure. On the cultivation ridges in both the NE and SE quadrants the most substantial oriented clay-lined pores are found in the top 4-5 cm of the soil. In the profile from the SE quadrant significant subordinate bands of readily detectable lined pores were also present at $13-14 \mathrm{~cm}$ and at $\mathrm{c} 18 \mathrm{~cm}$. In all the ridge profiles irregularly distributed patches of oriented clay-lined pores are present in decreasing numbers down to $21-24 \mathrm{~cm}$ below ridge surface. The actual number of oriented clay-lined pores present in the surface soil of an individual cultivation ridge may vary considerably within a short distance. For example, counts per thin section of 126 and 67 were obtained from the thin sections cut from duplicate samples taken within $30 \mathrm{~cm}$ of each other, but the distribution patterns within the group of ridge profiles are reasonably similar. In the deep SE quadrant profile further patches of oriented clay-lined pores (easily seen under low power stereomicroscope) were present in the gravel subsoil. Below the gravel a few small oriented clay-lined pores were present in the upper layers of the laminated silt down to $\mathrm{c} 177 \mathrm{~cm}$ below ridge surface.

In both of the profiles taken below the henge bank, pores lined with oriented clay, which are substantial enough to be easily seen under the low power stereomicroscope, are present down to 12 or $13 \mathrm{~cm}$ below the buried land surface.

The two thin sections from the central area have low counts of oriented clay-lined pores. The thin section from the entrance has substantial surface cracks and indentations infilled with oriented silt, and thin iron pan is absent. The other thin section from near the timber wall of the enclosure has a well developed iron pan c $35 \mathrm{~mm}$ below the surface. A few weak oriented silt-lined pores were seen above the iron pan and weak oriented clay-lined pores below; only one or two of these could be distinguished under the low power stereomicroscope.

The timber fence around the central area had been supported on the inside against side pressure from the surrounding ring bank by a revetment of soil dumped against it, and further material had washed down on top of the placed soil. Four samples taken at $30-35 \mathrm{~cm}$ intervals through the upper metre of this deposit contained substantial numbers (165-337) of oriented clay-lined pores. All the oriented clay linings were thick enough to be readily seen under the low power stereomicroscope. The scale of clay translocation in this revetting deposit is similar to that seen within the upper $2 \mathrm{~m}$ of the mound, and much greater than that observed in the buried soil profiles.

Six samples were taken from the ditch section on the $\mathrm{N}$ side of the mound. These samples spanned the lowest $37 \mathrm{~cm}$ of the ditch infill and about $11 \mathrm{~cm}$ of the underlying gravel. The ditch infill was very uniform. In the upper three thin sections peripheral bleached rims on stones were generally less than $0.1 \mathrm{~mm}$ wide and the stones were in all respects similar to those found in the mound turf and in the $A$ horizons of the buried soils. In the lowest layer of the ditch, bleached rims on stones were up to $0.4 \mathrm{~mm}$ wide and appeared to have been enhanced by gleying. Vertical root channels were infilled with fine silt. A few diatoms were present just above the ditch bottom. No pores infilled with oriented clay were seen in either the ditch infill material or the underlying gravel subsoil. These observations are consistent with a fairly rapid infill of the lower part of the ditch shortly after construction of the mound.

\section{DISCUSSION AND CONCLUSIONS}

In the prehistoric period the most essential characteristic of good arable land was that it should be flat, because prolonged cultivation reduced the organic matter content and structural cohesion of the soil, leaving it liable to downslope erosion after ploughing. Manageable soil texture and stone content, coupled with good under-drainage were also desirable. All these conditions were met at Strathallan and agricultural land use is proven by the presence of cultivation ridges below the mound. The presence of minimal bleached rims on the iron-stained peripheral weathering crusts around small stones in the surface layers of the buried soils below both henge bank and mound showed that stable profile morphology was retained throughout the interval between the construction of the henge bank and the mound. This must imply that there was regular replacement of the nutrients extracted by cropping. The development of a compacted $\mathrm{A}_{2} \mathrm{~g}$ horizon in the buried soils below both mound and henge, with a higher total phosphate content, coupled with the presence of soft thin iron pan on the cultivation ridges below the mound, suggest, as noted above, that domestic animals, probably sheep, were the source of the required input. This input probably represented a transfer of fertility from outfield grazings, a conclusion which has been 
strengthened by comparison with buried soils below the ramparts of the nearby Roman fort at Strageath, about $3 \mathrm{~km}$ further upstream, on the same terrace of the River Earn (Romans \& Robertson 1983). The ubiquitous presence of soft thin iron pan over the ground surface would suggest that the site was not under cultivation when the mound was built. But, as contemporary local observations have shown how quickly surface deterioration can be caused by grazing under unfavourable weather conditions, it would be unwise to assume this implied use as permanent pasture rather than temporary grazing fallow. When the total phosphate values for the buried profile below the SE quadrant (tables 11, 12) are plotted against depth, there is a straight line graph down to the maximum total phosphate value at $17-22 \mathrm{~cm}$ (or more) below the buried mineral ground surface. Previous work (Fairhurst \& Taylor 1971) has shown that phosphate can gradually leach down through the soil within archaeologically significant spans of time. The leaching rate for the Strathallan soil is not known, but experience elsewhere suggests that a rate somewhere between 20 and 40 years per centimetre may be applicable, and this would indicate that a stable pattern of agricultural activity had been maintained on the mound site for at least some hundreds of years before the mound was built.

The presence of small stones with iron-stained weathering crusts and weak peripheral bleached rims in the A horizon of the modern profile on top of the mound, indistinguishable from those present in the buried soils, was unexpected. But in this case the high total phosphate level (400-600 mg $\mathrm{P}_{2} \mathrm{O}_{5}$ per $100 \mathrm{~g}$ soil), the rather high level of exchangeable potassium and the former presence of mature trees on the mound combine to suggest that bird droppings have helped to counteract the effects of long-term leaching.

Before attempting to assess the significance of clay translocation and deposition within the mound and in the buried soils, some consideration must be given to the way in which colloidal suspension of clay may have been formed. It has often been noted that when a soil profile has been dug shortly after a prolonged period of rain, the flow of water from the saturated surface layers is at first milky with suspended clay as it runs down over the $\mathbf{B}$ and $\mathbf{C}$ horizons, but soon clears and flows clean thereafter. This demonstrates that clay is readily washed off recently cut surfaces, but that undamaged structure faces remain resistant and stable. It is therefore reasonable to suppose that within the mound mobilization of all the dispersible clay on mechanically damaged surfaces, followed by downwash and deposition in soil pores, occurred during the first period of prolonged really heavy rainfall after construction had been completed and before the dumped material had consolidated.

The presence of two clay deposition maxima within the mound is therefore strongly suggestive of two phases of construction. This is confirmed by the presence of strong iron and manganese mottling which has stained oriented clay deposited in soil pores at and above the interface between reddish brown turf material above and compact silt below. This mottling clearly postdates the formation of the lower clay maximum and is probably the result of surface-water gleying at the turf-silt interface which would become more impervious after the larger soil pores were infilled with oriented clay. This gleying must either predate the second phase of construction, because the clay deposition minimum just above (fig 66) indicates that water did not effectively penetrate much below $180 \mathrm{~cm}$ after the mound had been raised to its final height, or have occurred much later, after climatic deterioration during the Sub Atlantic period. The length of the interlude between construction phases is difficult to assess. It cannot have been a very long period as there was no visible indication of a buried soil surface in the lower part of the reddish brown turf deposit exposed during excavation of the SE quadrant. All things considered, the maximum estimate might be a few years and the minimum, given the right weather conditions, a few weeks.

The scale on which clay movement took place within the mound was far greater than in the buried soils. As mechanical disruption of soil dug up and used to build the mound has been identified as the basic reason for clay mobilization within the mound, this must point to cultivating implements as the most probable cause for clay mobilization and deposition within the surface layers of the buried soils. The dispersibility of these palaeo-cultivated soils was clearly enhanced by their poor structural condition and low organic matter content. This was c $1 \%$, as compared with $8-10 \%$ of organic matter in the plough layer of soils in the surrounding fields at the present day.

On the cultivation ridges there is maximum deposition of oriented clay in soil pores within the upper $4-5 \mathrm{~cm}$. Below the henge bank the corresponding depth is $\mathrm{c} 12 \mathrm{~cm}$. This may suggest shallow hoe cultivation on the raised ridges below the mound and ard ploughing on the flat land below the henge bank.

The silty palaeosurface soil has a rewelded fabric (Romans \& Robertson 1975), suggestive of consolidated worm-cast material, which extends right down to the interface with the gravelly subsoil below. This feature, together with the presence of scattered fragments of wood charcoal (including some identifiable fragments of oak charcoal), in the cultivation ridges, the mound turf material and the flat 


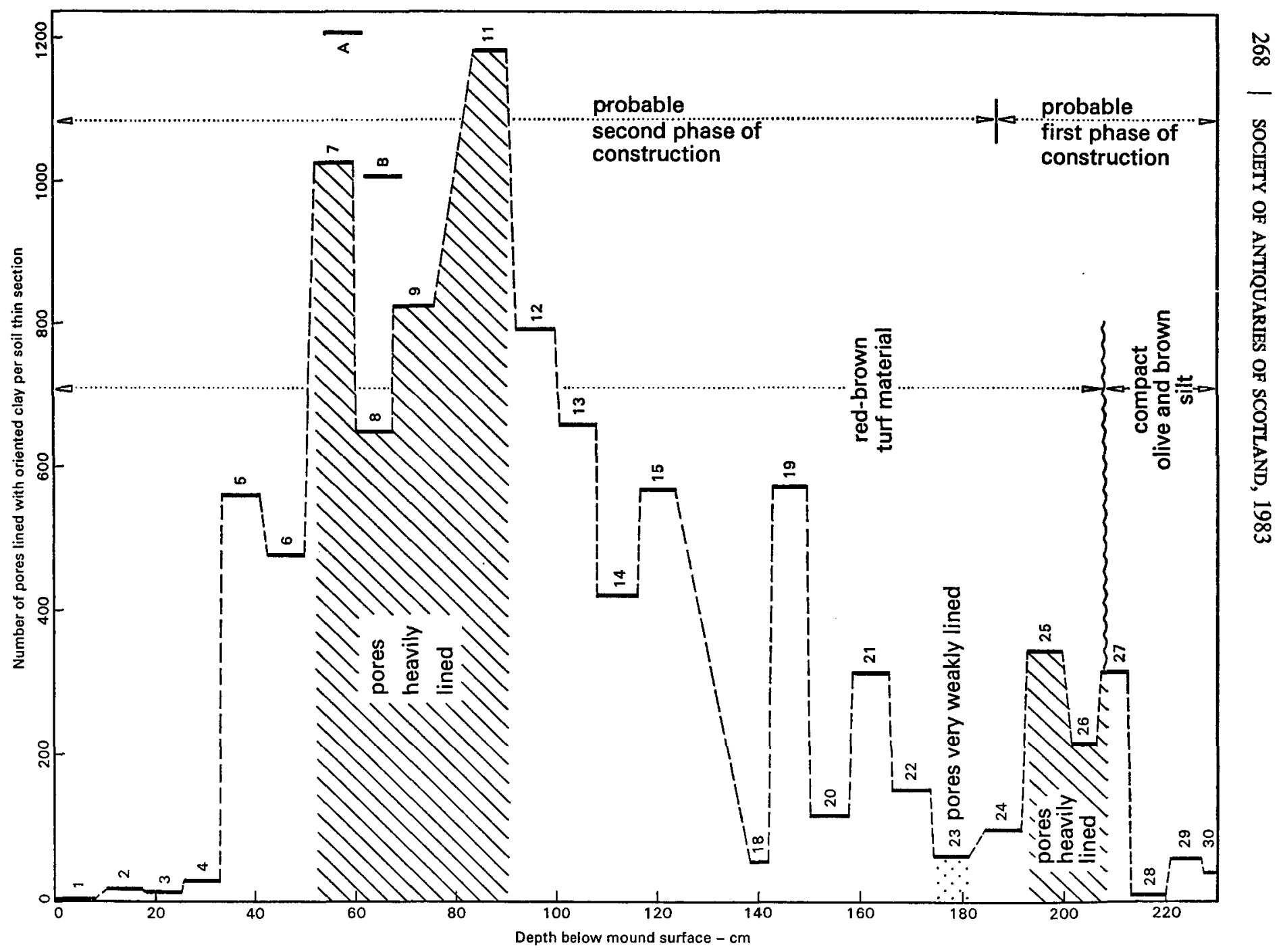

FIG 66 Distribution of pores lined with orientated clay throughout the upper $2.3 \mathrm{~m}$ of the mound at North Mains Farm, Strathallan (NB Thin sections 10,16 and 17 were omitted from this diagram as slide area was severely reduced during thin section preparation) 
land surface below the henge bank, indicates that a brown forest soil under mixed deciduous woodland was present on the Strathallan terrace before it was cleared and cultivated.

\section{PHOSPHATE SURVEYS ON THE HENGE, MOUND AND RING-DITCHES AT NORTH MAINS}

\section{P E Pare}

Soil samples were taken from three sites at North Mains duirng the course of excavation. On each site, a grid was laid out and samples taken, at an interval of $50 \mathrm{~cm}$ for the ring-ditches (on the gravel surface), $1 \mathrm{~m}$ under the barrow (on the OLS), and $2.5 \mathrm{~m}$ on the henge (on the gravel surface). The sample interval was selected to detect the suspected size of anomaly: presumably smaller for the ring-ditches than
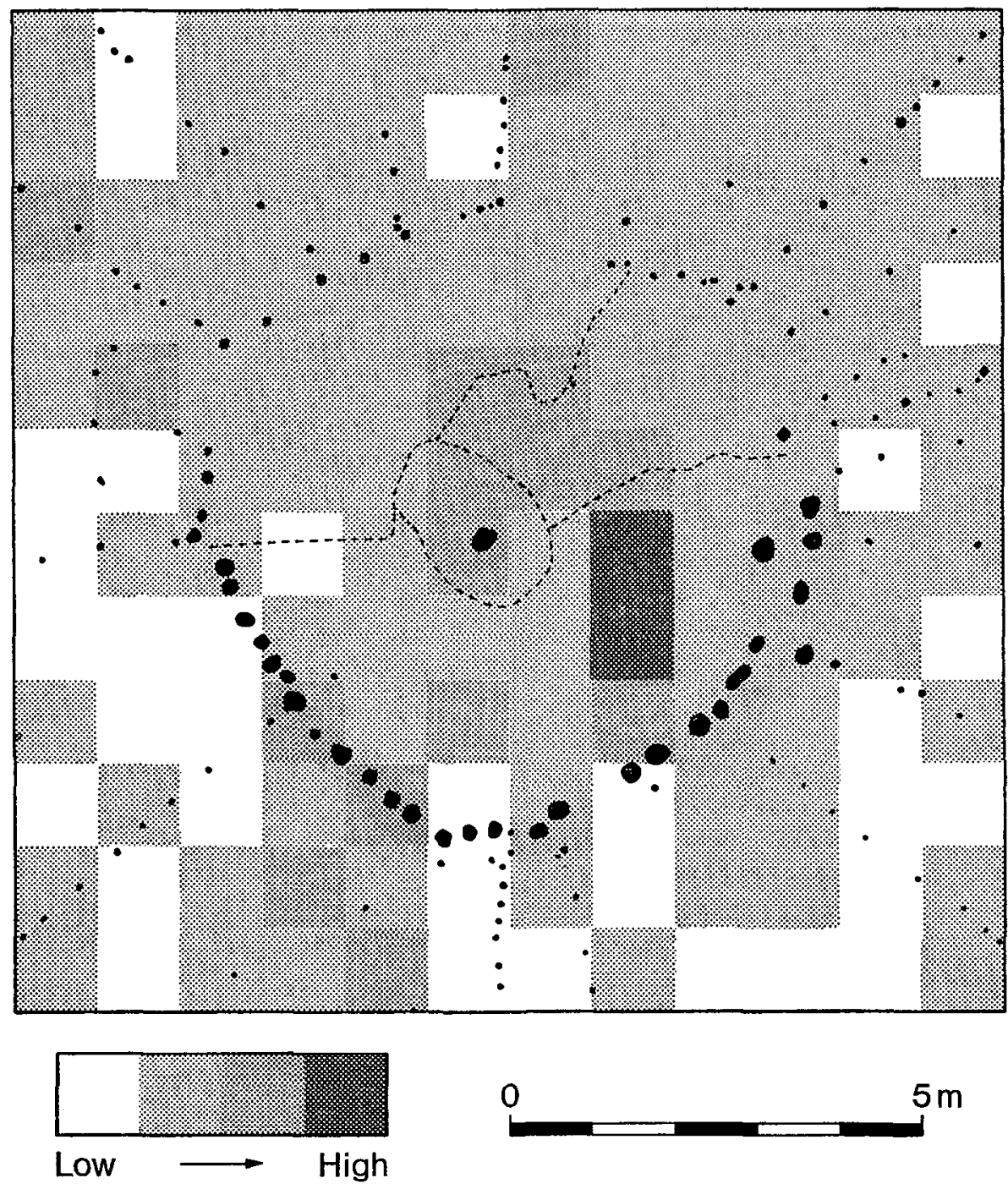

FIG 67 Barrow: central area phosphate survey results 
for the henge. In each of the grid squares, five samples were randomly selected and mixed, in order to obtain a representative phosphate level for the whole square. In all, 405 samples were analysed, including control samples taken outside the areas of proven prehistoric activity.

Each sample was air-dried and sieved, in readiness for laboratory treatment. The chemical analysis consisted of two stages, extraction of insoluble phosphates, and quantitative analysis of the solution obtained. The extraction was undertaken by treating the soil sample with $2 \mathrm{~N} \mathrm{HCl}$ for 2 hours in a simmering water bath. The resultant solution was filtered through phosphate-free filter paper and activated charcoal, and diluted. The dissolved phosphate was then stained with ammonium molybdate, using the Augustenburg method. The darkness of the solution thus produced is directly proportional to the phosphate concentration, and can be measured using a spectrometer (Egner et al 1960). Extraction with $2 \mathrm{~N} \mathrm{HCl}$, although not as rigorous as the method used for the phosphate survey at Balfarg Henge (Pare \& Nebelsick 1981), renders soluble most soil phosphates, and is fast and cheap (Provan 1972).

The results are mapped in fig 67 which shows the sampled area under the barrow, with the circular posthole and stakehole enclosure and bays. The $\mathbf{S}$ part of the area showed higher phosphate within the central area than outside, but this is not apparent in the $\mathrm{N}$ half. This difference between the two halves of the central area seemed to reinforce the two-phase interpretation for the enclosure, put forward by the excavator. It was, however, impossible to distinguish differences in the phosphate levels of different bays. Of considerable interest was the relatively high phosphate group over the central posthole and soil stain. Joined with this are two squares of very high phosphate unassociated with any traces of soil discoloration. Concerning these high phosphate concentrations within the central area, we may conjecture that this was caused by prehistoric activity within the enclosure: the collapsed remains of a structure, burials, or a combination of these and other causes.

No identification of prehistoric activity in the henge remained detectable in the phosphate record but interesting results were obtained from the two ring-ditches. In the case of ring-ditch 1, a definite phosphate gradient could be seen from NW to SE. For ring-ditch 2, no coherent pattern was visible, but the phosphate concentration was certainly very high compared with the area outside the ring.

I should like to thank Dr P Crooks of the Department of Agriculture, Edinburgh University, for help and advice concerning the chemical analyses.

\section{NOTE ON THE POLLEN CONTENT OF SOIL SAMPLES FROM THE MOUND AND HENGE AT NORTH MAINS}

\section{P D Hulme and J Shirriffs, Macaulay Institute for Soil Research}

During the course of the excavations soil samples were retained for pollen analysis. In the NE quadrant of the mound these consisted of a monolith, $35 \mathrm{~cm}$ deep, taken through the crest of a cultivation ridge exposed in the $\mathrm{N}$ baulk, and an additional sample from the same ridge-top, nearby. In the SE quadrant a similar monolith was taken from the wall of a profile pit close to the $E$ baulk. A sample was also collected from the $S$ facing side of the $E$ baulk. This consisted of material from the lower part of the $2 \mathrm{~m}$ thick mound capping of reddish brown turf. At the henge site samples were collected from two localities beneath the $\mathrm{N}$ section of the bank.

Samples were prepared for pollen counting using standard procedures (Faegri \& Iversen 1975; Moore \& Webb 1978). In addition, the NE quadrant and turf capping samples were treated with tetrasodium pyrophosphate (TSP), a recently developed method for deflocculating clay minerals (Bates et al 1978). Most samples contained very little pollen and the results are therefore presented as numbers of pollen grains (including fern and Sphagnum spores) counted rather than as conventional percentage diagrams. It must be emphasised that because of the scarcity of pollen, interpretation of the results is very tentative.

Pollen in the soil beneath the pre-ridge old land surface is extremely sparse (tables 15,16 ). Since the ridges were constructed from this soil they also, at the time of construction, would have contained very little pollen. Therefore, most of the pollen within the ridges probably belongs to the period between their construction and the commencement of mound building.

The pollen taxa from the NE quadrant ridge (table 15) indicate that in the vicinity of Strathallan there were areas of birch and alder (Betula, Alnus) and heathland (Ericoid, Cyperaceae) as well as agricultural areas (Plantago, Gramineae). The analysis of the separate ridge-top sample (table 17) shows 
TABle 15

Pollen analysis of NE quadrant soil monolith (results given as number of grains counted)

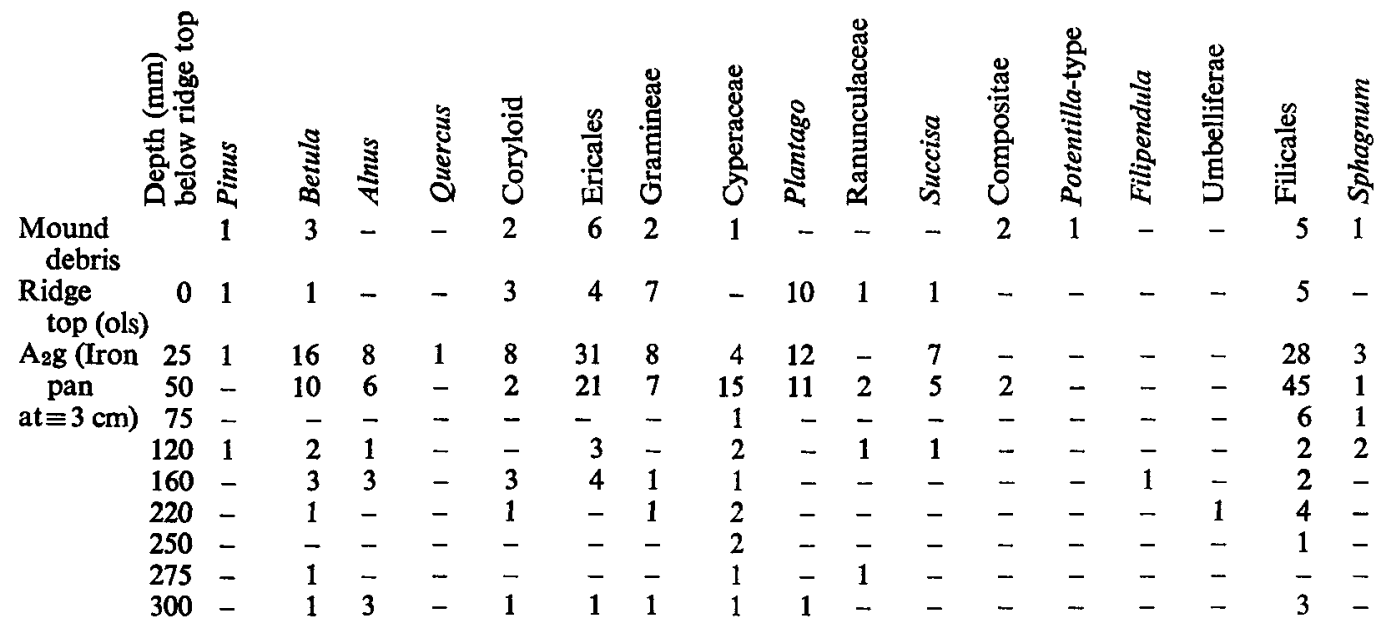

TABLE 16

Pollen analysis of SE quadrant soil monolith

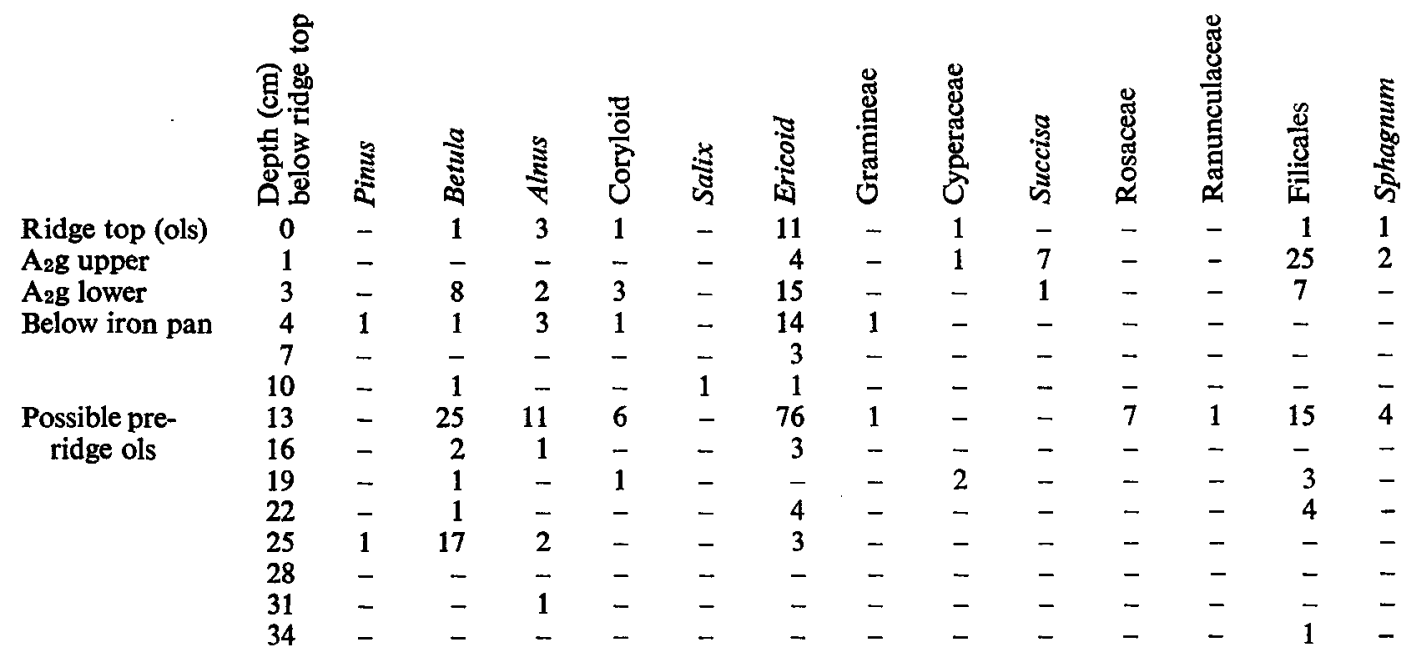

TABLE 17

Pollen analysis of separate soil samples from the mound

NE quadrant ridge top (ols)

SE quadrant turf capping sample

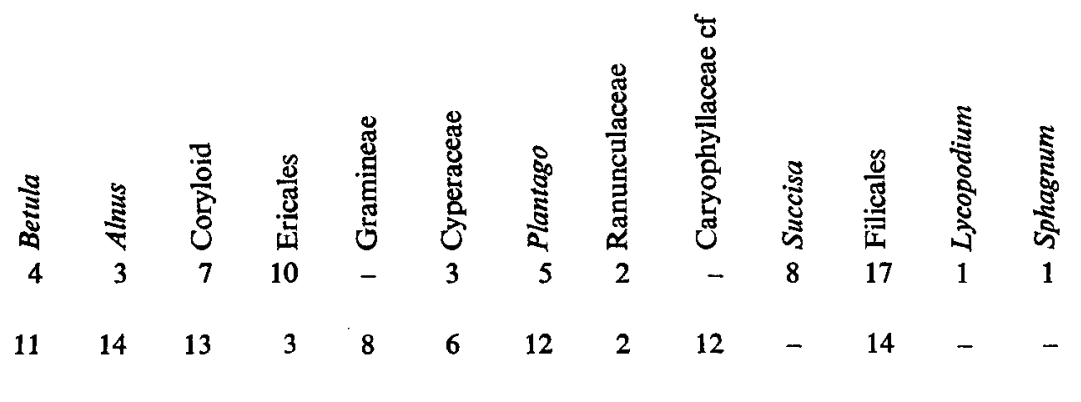


similar features except that no grass (Gramineae) pollen was identified. Results for the soil monolith from the SE quadrant show a relatively high pollen content at $13 \mathrm{~cm}$ (table 16) suggesting possible local survival of part of the pre-cultivation land surface. An analogous feature was not found in the NE quadrant monolith though it might have been present between sampling intervals. Within the cultivation ridge the tree and heathland taxa are again present but there is an almost total absence of agricultural indicators; in particular not one Plantago grain was recorded. It is reasonable to assume that this ridge was contemporary with the NE quadrant ridge, therefore the absence of agricultural indicators cannot be due to differences in pollen deposition or land-use. The samples from this monolith were not treated with TSP, but there is insufficient information about the effects of TSP treatment to establish that this was responsible for the observed differences. Pollen from the TSP-treated turf-capping sample from the SE quadrant (table 17) are generally comparable with the NE quadrant ridge, though in addition Plantago and the other indicator taxa are present.

The henge-bank buried soil (table 18) contained even less pollen than the soil below the mound. Only the site $2(3 \mathrm{~cm})$ sample gave a pollen count at all comparable with the ridge soils and no general conclusions can be drawn from this one sample. It is possible that increased oxidation as a result of ard ploughing may have reduced the pollen content of the henge soil as compared with the mound soil where shallow hoe cultivation has been suggested (see Soils Report, p 267).

TABLE 18

Pollen analysis of soil samples from the henge

\begin{tabular}{|c|c|c|c|c|c|c|c|c|c|c|c|}
\hline & 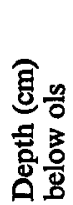 & క్ & 珰 & 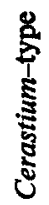 & 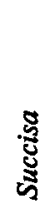 & 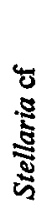 & نั & 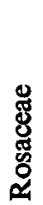 & 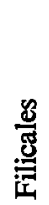 & 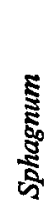 & 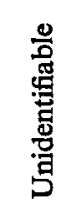 \\
\hline Site 1 & $\begin{array}{l}2 \\
5 \\
5\end{array}$ & $\overline{-}$ & $\overline{-}$ & - & $\begin{array}{l}\overline{1} \\
1\end{array}$ & $\overline{-}$ & $\begin{array}{l}- \\
- \\
-\end{array}$ & $\begin{array}{l}- \\
-\end{array}$ & $\begin{array}{l}9 \\
9 \\
4\end{array}$ & $\overline{1}$ & - \\
\hline Site 2 & $\begin{array}{l}3 \\
8\end{array}$ & 5 & $\begin{array}{r}4 \\
-\end{array}$ & $\begin{array}{l}3 \\
-\end{array}$ & 5 & - & 1 & 1 & $\begin{array}{r}18 \\
-\end{array}$ & - & 1 \\
\hline
\end{tabular}

Dimbleby (1961) suggests that 'where one finds a mixture of considerable proportions of both nontree species and tree species, the most likely explanation seems to be that there is a mosaic of vegetation types'. The pollen results for the turf capping and cultivation ridges would be consistent with such an interpretation but the available data are not really sufficient to be conclusive. However the indications suggest a Neolithic landscape in the Strathallan area not too dissimilar to that at present.

Pollen analysis of a $3.5 \mathrm{~m}$ core from a nearby kettle hole mire is in progress. It is hoped that this will give a fuller picture of the Neolithic vegetation types and the effect of the mound and henge builders on the vegetation. Preliminary results show that forest was dominant in the area from early Flandrian times (approximately 10,000 BP) and started to decline when pollen types associated with cultivation and land disturbance became relatively abundant. The start of the forest decline is expected to predate mound and henge construction by up to 1000 years. Full results of the pollen analysis supported by a macrofossil analysis and radiocarbon dates will be presented in a future report (Proceedings, 115).

\section{EXAMINATION OF PETROLOGY OF ARTEFACTS FROM THE HENGE, BARROW AND RING-DITCHES AT NORTH MAINS}

\section{G H Collins}

\section{CATALOGUE}

\section{Henge}

SF102 Diorite-erratic ?originally from Glen Lednock.

SF103 Medium grained red sandstone - LORS. 
SF104 Medium grained micaceous red sandstone LORS.

SF105 Medium grained micaceous green sandstone with quartz veinlets LORS.

SF106 Siltstone - probably of Lower Old Red Sandstone age.

SF107 Fine grained micaceous red sandstone LORS.

\section{Barrow}

SF113 Medium grained micaceous red sandstone LORS.

SF115 Siltstone probably indurated by proximity to an igneous intrusion LORS.

\section{Ring-ditches}

SF116 Pebble of quartzite.

\section{GENERAL REMARKS}

All the rocks present may have been derived quite locally to the site. Some, the igneous rocks in particular, are most probably glacial erratics.

\section{CONCLUSIONS}

\section{G J Barclay}

The three sites at North Mains illustrate fragments of ritual, burial and settlement from at least the mid third millennium bc to the mid first millennium ad. The ritual and burial aspects of the three sites, which overlap on the henge and barrow in the second millennium bc, necessitate the discussion of the monuments together.

The ring-ditches could not be dated but the pottery associations and radiocarbon date for F3 have provided a terminus post quem. It has been suggested (supra p 248) that they were funerary monuments of the later Neolithic in which one or more bodies placed on the old land surface were covered with the spoil from the ditches, with the possible addition of field stone or turf. The burial tradition represented has little in common with the rite discovered on the other two sites.

If the first major structure of the henge is accepted as the ring $A$, and bank and ditch complex then it is clear that the pre-bank features represent activity in or prior to the late third millennium. Excavation could not determine whether the Period I pits and burial A were part of a single phase of activity, perhaps the first organized 'ritual' use of the site, or represented sporadic and unrelated activities. It is certain only that Fl had been backfilled long enough before the erection of the bank to allow the development (? by agricultural activity) of a topsoil over it.

The pre-monument activity on the henge and barrow sites was largely agricultural. Both buried surfaces produced pedological evidence of tillage but the ribbing (representing spade or hoe cultivation) noted under the mound was not found under the henge bank. However, as it may not have extended far or may have been formed in the centuries between the henge and barrow, its absence on the henge need not be significant; Romans and Robertson have postulated ard cultivation on the henge. The quantities of pottery recovered from the old land surfaces and the body of the mound confirm activity in the area, though the artefact spread might reflect either the close proximity of a settlement, or midden and fertilizing practices.

The sub-old land surface features on the mound could not be dated. Their possible relationship with the henge or ring-ditches therefore cannot be tested. The pottery assemblage, as noted above, is of no value in clarifying the position.

Cairnpapple (Piggott 1948) in general provides a close parallel for the sequence of events at North Mains, including earlier activity, including burial, on a henge; its 'period I' pit setting 
with cremations is placed by Kinnes (1979) in his 'Stage F' (enclosed crematoria). He also suggests its association with the first cove structure. The open semi-circle of pits (? postholes) may also reflect the cove idea, and is, perhaps, broadly comparable with the later central setting at Croft Moraig and the semicircle of posts found, as the S part of the central enclosure, under the North Mains barrow.

Mercer (1981) has put forward a convincing argument for the relationship between the timber circle at Balfarg and recumbent stone circles (RSCs) of the north-east of Scotland, suggested by the increase in height of uprights to the SW. The interest in SW-NE lines evident in the Clava cairns, the ring-cairns of the north-east, the RSCs, and at Balfarg is not noticeable at the henge or mound at Strathallan, unless the NE quadrant access bay on the latter site is considered thus. It has already been suggested that the ring-bank of the barrow may be derived from the Clava ring-cairns, though an origin in Pitnacree type barrows might be more likely. Given the present suggested dating of the Clava ring-cairns to the late third millennium this development from the Clava passage graves may have been influenced by the Pitnacree type. In the $S$, stone annuli and pennanular banks have food vessel associations, as in a limited way, does North Mains barrow. Burl (1972) has pointed out the possible connection between the variety of Scottish henges which have continuous banks and the Scottish ring-cairn tradition. The broader relationships between the various forms of ritual and burial enclosures, from henges through ring-cairns, platform cairns, sub-barrow annuli and enclosed cemeteries, have been discussed at length elsewhere. This author would do no more than suggest that the mound might cover a semi-circular setting, possibly with its own independent ceremonial function, completed to a circle and surrounded by a ring-bank and used in this form for burial, all part of the general tradition of enclosure associated with ceremonial and burial of the late third and the second millennia bc, of which the henge is also a part.

The parallels between the generality of stone circles and the North Mains henge timber rings have been discussed above. Bryn Celli Ddu in Anglesey, a possible henge containing a fragmentary stone circle, has been placed by Kinnes in his 'Stage F', enclosed cemeteries, and is perhaps the best example of the early combination of these features - enclosure, free-standing rings of uprights, and burial - which combine and recombine throughout the Neolithic and the earlier Bronze Age in the henges, stone and timber circles (both with and without embanking), annular and penannular banks and cairns, annular and penannular timber settings under barrows, and the ring-cairns and related monuments. Combining with these is the practice or erecting round mounds, as at North Mains, or more simply at Dalgety and Cairnpapple.

The evidence from the henge and barrow suggests that on both sites there was some division of tasks in their construction. The bays formed by the radial fences under the barrow represent the organization of the work into units. Likewise ring A on the henge was erected in segments of straight lines of pits. However, the ditch of the barrow was segmented and probably dug by each bay-gang as required, while the henge ditch may have been dug by one gang each for the $\mathrm{N}$ and $\mathrm{S}$ halves (supra p 181). The bay system was abandoned near the top of the mound where it would have been impractical in the limited remaining space. The building of the mound, given its complex structural sequence, which was certainly planned from the first, would have required more organization than the simple task of erecting the bank of the henge. The significance of the possibly different methods of organization, and the potentially much smaller work force on the henge, can only be guessed at. MacKie (1977) has suggested that the henges were the work of a small specialist group. It must be said that the results of the North Mains excavation do not contradict this theory, but nor do they support it.

The secondary funerary use of both sites was broadly contemporary, which may account 
for the mound not being built on the henge. That is, the henge may have been in active ceremonial or burial use. The inhumations accompanied by food vessels would date, on present reckoning, from the 17 th to $14 / 13$ th centuries bc, as would the later beaker-associated activity. The urn burials could have been broadly contemporary or might be later but were probably part of the same general use of the henge.

The two inhumations dug into the flank of the mound, burials $E$ and $G$, can be compared with the henge inhumations; the rite, as it survives, is similar, and food vessel associations are common to both groups. Henge burials $\mathrm{B}$ and $\mathrm{C}$ differ from barrow burial $\mathrm{E}$ only in the depth of their pits. If henge burial $\mathrm{E}$ is an inhumation, then it is closely comparable to barrow burial $\mathrm{G}$, except for the part cover-slab in the latter.

The cremation deposits on the two sites compare less well. The unenclosed burials on the henge, A, L, M and $\mathrm{N}$, resemble burial $\mathrm{J}$ on the barrow, but as they all represent the simplest form of cremation burial and as $\mathbf{A}$ (henge) is probably at least 300 years earlier than $\mathbf{J}$ (barrow) this comparison is of little value. The remainder of the cremations on the barrow have no parallels on the henge, unless with burial $F$, the beaker-accompanied cremation. No urned cremations were found on the barrow, but burial $\mathrm{J}$ in a bipartite urn was the only multiple cremation on the henge. In contrast the majority of the barrow cremations were mulitple. Some inferences have been drawn from these deposits (supra $\mathrm{p} 240$ ) but without more precise dating the significance of the difference in rite cannot be assessed.

The large number of children in the cremation deposits on the barrow (very approximately 15 , from neonatal to adolescence) plus barrow inhumation $\mathrm{E}$, contrast with the single child on the henge (in multiple burial $\mathrm{J}$ ). Could the children of one group be buried in one place while adults had a choice of two, or are two groups represented, one with a different rite for children or can the difference be explained merely as a chronological function? Burgess (1980, 297) has noted that inhumation and cremation are choices within one tradition and that the rite and the site might be decided by individual family or community custom or convention. There need be no recourse to chronology to account, entirely, for the differences seen in the two sites.

The funerary use of the area seems to have continued into the first millennium bc with the pyre burials ( $\mathrm{p}$ 143) to the $\mathrm{N}$ of the site, definitely human in the case of burial O in the top of F6 and probably human in the upper parts of F3 and F5. The possible association of enclosed cremation cemeteries and ring-ditch features, themselves commonly enclosing cremation deposits (including in situ cremation) and the henge tradition have been mentioned above. The pyre burial rite outside the enclosure may show, in a way, the attraction of the vicinity of an earlier monument for people practising a later form of the tradition. It is perhaps significant that the henge enclosure was avoided.

The prehistoric use of the henge and barrow spans a period of over 1000 years. Earlier occupation is proved by the extended agricultural activity found under mound and henge, the radiocarbon dates of F3 at the ring-ditches and the scatter of 'Western Neolithic' pottery. The whole range of ritual activities is, however, isolated from the contemporary settlement evidence, apart from the buried cultivated soils. A great deal of land was sacrificed to the two larger monuments (henge: $c 3850 \mathrm{~m}^{2}$; barrow: $2700 \mathrm{~m}^{2}$ ) though in the case of the barrow some of the loss might have been made up by the removal of field clearance heaps for building the stone elements of the mound. Both areas were under pasture at the time of the construction of the monument though both areas had been tilled; perhaps this indicates the rotation of crops or the movement of settlement.

The method used to organize the labour on the mound hints at the involvement of perhaps 100 people. Without some evidence of the settlement size, this mound, in a period when single, 
or small multiple, burials were the norm and barrows were small, cannot be explained within the present view of the northern early Bronze Age. The mound was one of the largest round barrows in Scotland, comparable only with the Orkney-Cromarty tombs and the largest of the barrows of Strathtay; none of those in the Earn and Allan valleys approach it. The Strathtay monuments, which were generally dated to the early third millennium by the results of the Pitnacree excavation (Coles \& Simpson 1965) might now be considered as spanning a rather longer period. The North Mains barrow and many of the large barrows in the region are likely to be a late but very vigorous reflection of the burial traditions of the mid to late Neolithic. However, a strong element of late Neolithic and early Bronze Age ceremonial practice can be seen in the structure.

It is to be hoped that the pollen core will cast a little more light on the less esoteric activities of the populace and that the opportunity to excavate a settlement of the late Neolithic-Early Bronze Age in the area will present itself, to allow the ritual sites to be seen within a firmer economic and social pattern.

\section{ACKNOWLEDGEMENTS}

My greatest thanks go to all those who worked at North Mains: James Rideout, assistant supervisor, seasons 2 and 3; Jill Kendrick, assistant supervisor, season 1; and the site assistants, John Cannell, Jean Comrie, Jill Harden, John Hardisty, John Price, Margaret Rideout and Nick Tavener, and the volunteers, of whom more than 200 worked on the site. I would also like to thank Sir William Roberts and his staff at Strathallan for their generous assistance throughout the excavation. Sir William kindly provided accommodation throughout the project and is also to be thanked for his permission to excavate the sites.

James Rideout, in addition to his skilful work on the site illustrations, has provided invaluable help in the preparation of the report; he has acted as a critical sounding-board for my interpretations of the sites and has contributed many ideas. My thanks to him for his constant support, enthusiasm and hard work in the five years since North Mains was begun. Without David Breeze, then head of the CEU, the project could not have been carried to completion; I give my thanks for his encouragement, support and, when necessary, stern compulsion. My great thanks are also due to the many people who worked on the North Mains material, especially my co-authors and illustrators listed on $\mathrm{p}$ 122, and David Heppel and William Lindsay who contributed short notes on a shell fragment and the medieval pottery respectively. Other post-excavation work was carried out by Rosemary Cowie, who cleaned and consolidated the finds, after preliminary work by Margaret Rideout, and, under the supervision of Richard Welander of IAM laboratory, painstakingly reconstructed the vessels; by John Barber, who identified the charcoal from the sites; and by Jenny Lee, Jill Kendrick, Sharon Dixon and Derek Hall.

My colleagues in the CEU and in the Ancient Monuments Division generally have been a constant source of help; John Barber, Michael Brooks (who processed the photographic record and prepared the plates for publication), Patrick Ashmore, Suzzanne Hunter, Gerry Kennedy, the staff of the various administrative sections, and the Motor Transport Unit.

I would like to express my great thanks to those who have given so much time to reading and commenting on the manuscript, to its great benefit: Trevor Cowie, Graham Ritchie, Roger Mercer, David Breeze, James Rideout and John Barber; all remaining imperfections are, however, entirely my responsibility. All of the above also gave valuable advice on site. Professor Christopher Hawkes kindly contributed comments on the function of food vessels. I would like to thank the archaeologists not already mentioned who visited the site and provided stimulating discussion, most notably Lionel Masters. 
Throughout the excavation plant was supplied by J M Dewar of Muthill; he and his staff are to be complimented on their skill and professionalism. I am grateful to the Royal Commission on the Ancient and Historical Monuments of Scotland for permission to reproduce pl 9.

\section{REFERENCES}

Alexander, J E 1868 'Opening of the Fairy Knowe of Pendreich, Bridge of Allan,' Proc Soc Antiq Scot, 7 (1867-8), 519-23.

ApSimon, A M 1949 'Gorsey Bigbury, the second report', Proc Univ Bristol Spel Soc, 6 (1949), 186-99.

Ashbee, P 1958 'The excavation of Tregulland Burrow, Treneglos Parish, Cornwall', Antiq J, 38 (1958), 174-96.

Ashbee P 1960 The Bronze Age Round Barrow in Britain. London.

Ashbee, P 1966 'The Fussell's Lodge long barrow excavations, 1957', Archaeologia, 100 (1966), 1-80.

Ashbee, P 1970 The Earthen Long Barrow in Britain. London.

Ashbee, P, Smith, I F \& Evans, J G 1979 'Excavation of three long barrows near Avebury, Wilts', Proc Prehist Soc, 45 (1979), 207-300.

Atkinson, R J C, Piggott, C M \& Sandars, N K 1951 Excavations at Dorchester, Oxon. Oxford.

Barber, J 1958 The Middle Bronze Age Cinerary Urns of Scotland. MA dissertation, Dept of Archaeology, University of Edinburgh.

Barber, J W A 1978 'The excavation of the holed stone at Ballymeanoch, Kilmartin, Argyll', Proc Soc Antiq Scot, 109 (1977-8), 104-12.

Barber, J W A forthcoming Excavations on Arran 1978-81.

Barclay, G J 1980 North Mains, Strathallan - the excavation of the barrow, henge and ring-ditches 1978-9 - interim report. Privately circulated paper, SDD (Ancient Monuments), Central Excavation Unit, Edinburgh.

Barclay, G J 1982 'Late Neolithic and early Bronze Age burials: their treatment in salvage and rescue archaeology', Scot Archaeol Rev, 1 (1982), 21-3.

Bates, C D, Coxen, P \& Gibbard, P L 1978 'A new method for the preparation of clay-rich sediment samples for palynological investigations', New Phytol, 81 (1978), 459-63.

Bradley, R 1978 The Prehistoric Settlement of Britain. London.

Bradley, R \& Keith-Lucas, M 1975 'Excavation and pollen analysis on a bell barrow at Ascot, Berkshire', J Archaeol Sci, 2 (1975), 95-108.

Buchanan, M \& Callander, J G 1923 'Report on a Bronze Age grave and two others discovered last year at Camelon, Stirlingshire; with a note on the relics found', Proc Soc Antiq Scot, 57 (1922-3), 243-50.

Burgess, C 1974 'The bronze age', in Renfrew, C (ed), 165-232.

Burgess, C 1980 The Age of Stonehenge. London.

Burl, H A W 1969 'Henges: internal features and regional groups', Archaeol J, 126 (1969), 1-28.

Burl, H A W 1972 'Stone circles and ring cairns', Scot Archaeol Forum, 4 (1972), 31-47.

Burl, H A W 1976 The Stone Circles of the British Isles. London.

Callander, J G 1924 'A short cist containing a food-vessel and human remains at Bridgeness, West Lothian', Proc Soc Antiq Scot, 58 (1923-4), 286-94.

Callander, J G 1928 'A beaker from a short cist in a long cairn at Kilmarie, Skye', Proc Soc Antiq Scot, 62 (1927-8), 22-6.

Callander, J G 1929 'Scottish Neolithic pottery', Proc Soc Antiq Scot, 63 (1928-9), 29-98.

Case, H J 1952 'The excavation of two round barrows at Poole, Dorset', Proc Prehist Soc, 18 (1952), $148-59$.

Case, H J 1977 'The beaker culture in Britain and Ireland', in Mercer, R J (ed), Beakers in Britain and Europe, Oxford, 71-101. (=Brit Archaeol Rep Int Ser, 26).

Catherall, P D 1974 Henge Monuments - Origins and Functions. MA thesis, Dept of Ancient History, University of Sheffield.

Catherall, P D 1976 'Henge monuments: monument or myth?', in Burgess, C and Miket, R (eds) Settlement and Economy in the Third and Second Millennia BC, Oxford, 1-9. (= Brit Archaeol Rep Brit Ser, 33). 
Christie, P M 1967 'A barrow-cemetery of the second millennium BC in Wiltshire, England', Proc Prehist Soc, 33 (1967), 336-66.

Clark, J G D 1932 'The date of the plano-convex flint-knife in England and Wales', Antiq J, 12 (1932), $158-62$.

Clark, J G D 1934 'Derivative forms of the petit tranchet in Britain', Archaeol, J, 91 (1934), 32-59.

Clark, J G D 1936 'The timber monument at Arminghall and its affinities', Proc Prehist Soc, 2 (1936), 1-51.

Clarke, D L 1970 The Beaker Pottery of Great Britain. Cambridge.

Coles, F R 1900 'Stone circles in Kincardineshire (North) and part of Aberdeenshire', Proc Soc Antiq Scot, 34 (1899-1900), 139-98.

Coles, F R 1906 'Notices of standing stones, cists and hitherto unrecorded cup-and-ring marks in various localities', Proc Soc Antiq Scot, 40 (1905-6), 291-327.

Coles, J M 1976 'A Neolithic hurdle from the Somerset Levels', Antiquity, 50 (1976), 57-61.

Coles, J M \& Simpson, D D A 1965 'The excavation of a neolithic round barrow at Pitnacree, Perthshire, Scotland', Proc Prehist Soc, 31 (1965), 34-57.

Cowie, T G 1978a Bronze Age Food Vessel Urns. Oxford. (=Brit Archaeol Rep Brit Ser, 55).

Cowie, T G 1978b 'Excavations at the Catstane, Midlothian, 1977', Proc Soc Antiq Scot, 109 (1977-8), 166-201.

Craw, J H 1914 'Account of the excavation of two Bronze Age cairns in the parish of Foulden ... Berwickshire', Proc Soc Antiq Scot, 48 (1913-4), 316-33.

Croft Andrew, C K 1948 'Recent archaeological work in Cornwall', Trans Plymouth Inst, 20 (1948), $39-46$.

Crompton, E 1966 Soils of the Preston District of Lancashire. (=Mem Soil Survey of Great Britain).

Cummins, W A 1979 'Neolithic stone axes: distribution and trade in England and Wales', in Clough, T H McK \& Cummins, W A (eds), Stone Axe Studies, London, 5-12. (= Council Brit Archaeol Res Rep, 23).

Dalrymple, C E 1884 'Notes on the excavation of the stone circle at Crichie, Aberdeenshire', Proc Soc Antiq Scot, 18 (1883-4), 319-25.

Davidson, J M 1935 'A Bronze Age cemetery at Knappers, Kilbowie, Dunbartonshire', Proc Soc Antiq Scot, 69 (1934-5), 352-86.

Dickson, J H 1978 'Bronze age mead', Antiquity, 52 (1978), 108-13.

Dimbleby, G W 1961 'Soil pollen analysis', J Soil Sci, 12 (1961), 1-11.

Dudley, D 1961 'The excavation of the Otterham barrow, Cornwall', J Roy Inst Cornwall, 4 (1961), 62-80.

Edwards, C A \& Lofty, J R 1972 Biology of Earthworms. London.

Edwards, C A \& Lofty, J R 1974 'Invertebrate fauna of the park grass plots, Rothamsted Experimental Station', Res Report for 1974, 133-54.

Egner, H, Riehm, H \& Domingo, W R 1960 'Untersuchungen über die chemische Bodenanalyse als Grundlage für Beurteilung des Nahrstoffzustandes der Boden. II Chemische Extractionsmethoden zur Phosphor-und Kalium-bestimmung', Kungl Lantbrukskögskolans Ann, 26 (1960), 199-215.

Evans, J 1897 The Ancient Stone Implements, Weapons and Ornaments of Great Britain, 2nd ed. London.

Evans, J G, Limbrey, S \& Cleere, H 1975 The Effect of Man on the Landscape: the Highland Zone. London. (= Counc Brit Archaeol Res Rep, 11).

Faegri, K \& Iversen, J 1975 Textbook for Pollen Analysis, 3rd ed. Oxford.

Fairhurst, H \& Taylor, D T 1971 'A hut-circle settlement at Kilphedir, Sutherland', Proc Soc Antiq Scot, 103 (1970-1), 65-99.

Fox, C 1937 'Two Bronze Age cairns in S Wales: Simondstown and Pond Cairns Coity Higher Parish, Bridgend', Archaeologia, 87 (1937), 129-80.

Fox, C 1959 Life and Death in the Bronze Age. London.

Galloway, T L 1920 'Prehistoric Argyll - report on the exploration of a burial cairn at Balnabraid, Kintyre', Proc Soc Antiq Scot, 54 (1919-20), 172-191.

Gemmell, A M D \& Kesel, R H 1979 Development in the study of the Buchan flint deposits, Scot Archaeol Forum, 9 (1979), 66-77. 
Gibson, A 1978 Bronze Age Pottery in the North-East of England. Oxford. (=Brit Archaeol Rep Brit Ser, 56).

Green, H S 1980 The Flint Arrowheads of the British Isles. Oxford. (=Brit Archaeol Rep Brit Ser, 75). Greenwell, W 1866 'An account of excavations in cairns near Crinan', Proc Soc Antiq Scot, 6 (1864-6), 336-51.

Harding, A F 1981 'Excavations in the prehistoric ritual complex near Milfield, Northumberland', Proc Prehist Soc, 47 (1981), 87-136.

Hardy, J 1889 'On British urns found at Hoprig near Cockburnspath, Berwickshire', Berwickshire Nat Club, 12 (1887-9), 131-7.

Hawkes, C 1977 'Some further thoughts', Ir Archaeol Res Forum, 4 (1977), 39.

Hawkes, C 1981 'Alcoholic food vessels?', Current Archaeol, 79 (1981), 255.

Henshall, A S 1963 The Chambered Tombs of Scotland, vol 1. Edinburgh.

Henshall, A S 1972 The Chambered Tombs of Scotland, vol 2. Edinburgh.

Henshall, A S 1983 'The Neolithic pottery from Easterton of Roseisle, Moray', in Clarke, D V \& O'Connor, A (eds), From the Stone Age to the Forty-five, Edinburgh, 19-44.

Houlder, C 1968 'The henge monuments at Llandegai', Antiquity, 42 (1968), 216-21.

Jobey, G \& Tait, J 1966 'Excavations on palisaded settlements and cairnfields at Alnham, Northumberland', Archaeol Aeliana, 4 ser, 44 (1966), 5-48.

Kenworthy, J B 1972 'Ring-cairns in north-east Scotland', Scot Archaeol Forum, 4 (1972), 18-30.

Kenworthy, M 1977 Timber Circles Under British Round Barrows. MA dissertation, Dept of Prehistoric Archaeology, University of Edinburgh.

Kilbride-Jones, H E 1935 'An account of the excavation of the stone circle at Loanhead of Daviot, and of the standing stones of Cullerlie, Echt, both in Aberdeenshire', Proc Soc Antiq Scot, 69 (1934-5), $168-223$.

Kilbride-Jones, H E 1936 'Late Bronze Age cemetery: being an account of the excavations of 1935 at Loanhead of Daviot, Aberdeenshire', Proc Soc Antiq Scot, 70 (1935-6), 278-310.

Kinnes, I 1979 Round Barrows and Ring-ditches in the British Neolithic. London. (=Brit Mus Occ Paper, 7 ).

Kirk, W \& MacKenzie, J 1955 'Three Bronze Age cist burials in NE Scotland', Proc Soc Antiq Scot, 88 (1954-5), 1-14.

Lacaille, A D 1940 "Aspects of intentional fracture (being notes on the flaking of some rocks other than fint as exemplified by some Scottish artifacts)', Trans Glasgow Archaeol Soc, 9 (4) (1937-40), 313-41.

Lanting, J N \& van der Waals, J D 1972 'British beakers seen from the Continent', Helinium, 12 (1972), $20-46$.

Leaf, C S 1940 'Further excavations in Bronze Age barrows at Chippenham, Cambridgeshire', Proc Cambridge Antiq Soc, 39 (1940), 29-68.

Longworth, I H 1961 'The origins and development of the primary series in the collared urn tradition in England and Wales', Proc Prehist Soc, 27 (1961), 263-306.

Longworth, I H 1967 'Further discoveries at Brackmont Mill and Tentsmuir, Fife', Proc Soc Antiq Scot, 99 (1966-7), 60-92.

Lynch, F (ed) 1975 'Brenig Valley excavations 1974 - interim report', Denbighshire Hist Trans, 24 (1975), 1-25.

McAdam, E 1974 Some Aspects of Early Bronze Age Short Cists. MA Dissertation, Dept of Archaeology, University of Edinburgh.

McInnes, I J 1964 'The Neolithic and Early Bronze Age pottery from Luce Sands, Wigtownshire', Proc Soc Antiq Scot, 92 (1963-4), 40-81.

McInnes, I J 1969 'A Scottish Neolithic pottery sequence', Scot Archaeol Forum, 1 (1969), 19-30.

MacKie, E W 1977 Science and Society in Prehistoric Britain. London.

MacLaren, A 1967 'Recent excavations in Peeblesshire', Proc Soc Antiq Scot, 99 (1966-7), 93-104.

Manby, T G 1974 Grooved Ware Sites in the North of England. Oxford. (=Brit Archaeol Rep Brit Ser, 9).

Mann, L M 1902 'Note of the finding of an urn, jet necklace, stone axe and other associated objects in Wigtownshire', Proc Soc Antiq Scot, 36 (1901-2), 584-9.

Mercer, R J 1981 'The excavation of a late neolithic henge-type enclosure at Balfarg, Markinch, Fife, Scotland', Proc Soc Antiq Scot, 111 (1981), 63-171.

Miles, H 1973 'Caerloggas, St Austell', Cornish Archaeology, 12 (1973), 56.

Moore, P D \& Webb, J A 1978 An Illustrated Guide to Pollen Analysis. London. 
Morrison, A 1968 'Cinerary urns and Pygmy vessels in South-West Scotland', Trans Dumfriesshire Galloway Nat Hist Antiq Soc, 3 ser, 45 (1968), 80-140.

O'Neill, H E 1967 'Bevan's Quarry round barrow, Temple Guiting, 1964', Trans Bristol Glos Archaeol Soc, 86 (1967), 16-41.

O'Riordain, S P 1940 'Excavations at Cush, Co. Limerick', Proc Roy Ir Acad, C, 45 (1940), 83-131.

Pare, C F E \& Nebelsick, L D 1981 'The phosphate report', in Mercer 1981, 146-7.

Pierpoint, S 1980 Social Patterns in Yorkshire Prehistory 3500-750 BC. Oxford. (=Brit Archaeol Rep Brit Ser, 74).

Piggott, C M 1938 'A Middle Bronze Age barrow and Deverel-Rimbury urnfield at Latch Farm, Christchurch, Hampshire', Proc Prehist Soc, 4 (1938), 169-87.

Piggott, S 1931 'The Neolithic pottery of the British Isles', Archaeol J, 88 (1931), 67-158.

Piggott, S 1948 'The excavations at Cairnpapple Hill, West Lothian, 1947-8', Proc Soc Antiq Scot, 82 (1947-8), 68-123.

Piggott, S 1955 'Excavations in passage graves and ring cairns of the Clava group, 1952-3', Proc Soc Antiq Scot, 88 (1954-5), 173-207.

Piggott, S \& Piggott, C M 1939 'Stone and earth circles in Dorset', Antiquity, 13 (1939), 138-58.

Piggott, S \& Powell, T G E 1949 'The excavation of three Neolithic chambered tombs in Galloway, 1949', Proc Soc Antiq Scot, 83 (1948-9), 103-61.

Piggott, S \& Simpson, D D A 1971 'Excavation of a stone circle at Croft Moraig, Perthshire, Scotland', Proc Prehist Soc, 37 (1971), 1-15.

Provan, D M J 1972 'Soil phosphate analysis as a tool in archaeology', Norwegian Archaeol Rev, 5 (1972), 37-50.

Rahtz, P A 1962 'Farncombe Down barrow, Berkshire', Berks Archaeol J, 60 (1962), 1-24

Renfrew, C (ed) 1974 British Prehistory-A New Outline. London.

Ritchie, J 1920 'The stone circle at Broomend of Crichie, Aberdeenshire', Proc Soc Antiq Scot, 54 (1919-20), 154-72.

Ritchie, J N G 1972 'Excavation of a chambered cairn at Dalineun, Lorn, Argyll', Proc Soc Antiq Scot, 104 (1971-2), 48-62.

Ritchie, J N G 1974 'Excavation of the stone circle and cairn at Balbirnie, Fife', Archaeol J, 131 (1974), 1-32.

Ritchie, J N G 1976 'The stones of Stenness, Orkney', Proc Soc Antiq Scot, 107 (1975-6), 1-60.

Ritchie, J N G \& MacLaren, A 1972 'Ring-cairns and related monuments in Scotland', Scot Archaeol Forum, 4 (1972), 1-17.

Ritchie, J N G, Thornber, I, Lynch, F \& Marshall, D N 1975 'Small cairns in Argyll: some recent work', Proc Soc Antiq Scot, 106 (1974-5), 15-39.

Romans, J C C \& Robertson, L 1975 'Some genetic characteristics of the freely drained soils of the Ettrick association', Geoderma, 14 (1975), 297-317.

Romans, J C C \& Robertson, L 1983 'The general effects of early agriculture on the soil profile', in Maxwell, G S (ed), The Impact of Aerial Reconnaissance on Archaeology, London, 136-41. (= Counc Brit Archaeol Res Rep, 49).

Scott, J 1977 'The Rothesay style of Neolithic pottery in Scotland', in Marshall, D N \& Taylor, I, 'The excavation of the chambered cairn at Glenvoidean, Isle of Bute', Proc Soc Antiq Scot, 108 (1976-7), 1-39.

Sharples, N M 1981 'The excavation of a chambered cairn, the Ord North, at Lairg, Sutherland, by JXWP Corcoran', Proc Soc Antiq Scot, 111 (1981), 21-62.

Shepherd, W 1972 Flint: Its Origin, Properties and Uses. London.

Simpson, D D A 1968 'Food vessels: associations and chronology', in Coles, J M \& Simpson, D D A (eds), Studies in Ancient Europe, Leicester, 197-211.

Smith, I F 1965 Windmill Hill and Avebury: Excavations by Alexander Keiller 1925-1939. Oxford.

Smith, I F 1974 'The Neolithic', in Renfrew 1974, 100-36.

Stevenson, J B 1975 'Survival and discovery', in Evans et al 1975, 104-8.

Stevenson, R B K 1947 “"Lop-sided” arrowheads', Proc Soc Antiq Scot, 81 (1946-7), 179-82.

Stewart, M E C 1973. 'Almondbank short cist cemetery, 'Discovery Excav Scot 1973, 40-1.

Stewart, M E C 1974 'Moncrieffe stone circle and henge', Discovery Excav Scot 1974, 86-7.

Stuart J 1857 'Notice of sculptured stones and other antiquities found on the Castle Hill of Kintore, and of other ancient remains found in the neighbourhood', Proc Soc Antiq Scot, 2 (1854-7), 230-3. 
Thomas, C 1971 The Early Christian Archaeology of North Britain. Oxford.

Thorpe, O N \& Thorpe, R S 1984 'The distribution and sources of archaeological pitchstone in Britain', J Archaeol Sci, 11 (1984), 1-34.

Triscott, J 1982 'Excavations at Dryburn Bridge, East Lothian, 1978-9', in Harding, D W (ed), Later Prehistoric Settlement in South-East Scotland, Edinburgh, 117-24. (=Univ of Edinburgh, Dept of Archaeology, Occ Paper, 8).

Tyson, N 1972 A Bronze Age Cairn at Wind Hill, Heywood, Lancs. Bury.

Varley, W J 1938 'The Bleasdale circle', Antiq J, 18 (1938), 154-71.

Wainwright, G J 1969 'A review of henge monuments in the light of recent research', Proc Prehist Soc, 35 (1969), 112-33.

Wainwright, G J \& Longworth, I H 1971 Durrington Walls: Excavations 1966-68. London. (=Rep Res Comm Soc Antiq Lond, 29).

Ward, J 1970 Excavation of a barrow at Broughton Knowe, Peeblesshire, Proc Soc Antiq Scot, 102 (1969-70), 101-4.

Watkins, T F 1982 The excavation of an Early Bronze Age cemetery at Barns Farm, Dalgety, Fife', Proc Soc Antiq Scot, 112 (1982), 48-141.

Watkins, T F \& Shepherd, I A G 1980 'A beaker burial at Newmill, near Bankfoot, Perthshire', Proc Soc Antiq Scot, 110 (1978-80), 32-43.

Wickham-Jones, C R \& Collins, G 1978 'The flint and chert sources of Northern Britain', Proc Soc Antiq Scot, 109 (1977-8), 7-21.

Winning, J G 1886 'Notice of a cist containing an urn found at Eckford, Roxburghshire and opened on 31 October 1885', Berwickshire Nat Club, 11 (1885-6), 179-81.

Yates, C G \& Jackson, S 1900 'Bronze Age burials on Fairsnape Farm, Bleasdale, Lancs', Reliquary, ns $6(1900), 258-63$.

Young, H W 1895 'Cinerary urn found near Buckie, in Banffshire', Reliquary, ns 1 (1895), 229-30.

This paper is published with the aid of a grant from the Scottish Development Department 
PSAS 113 | PLATE 9

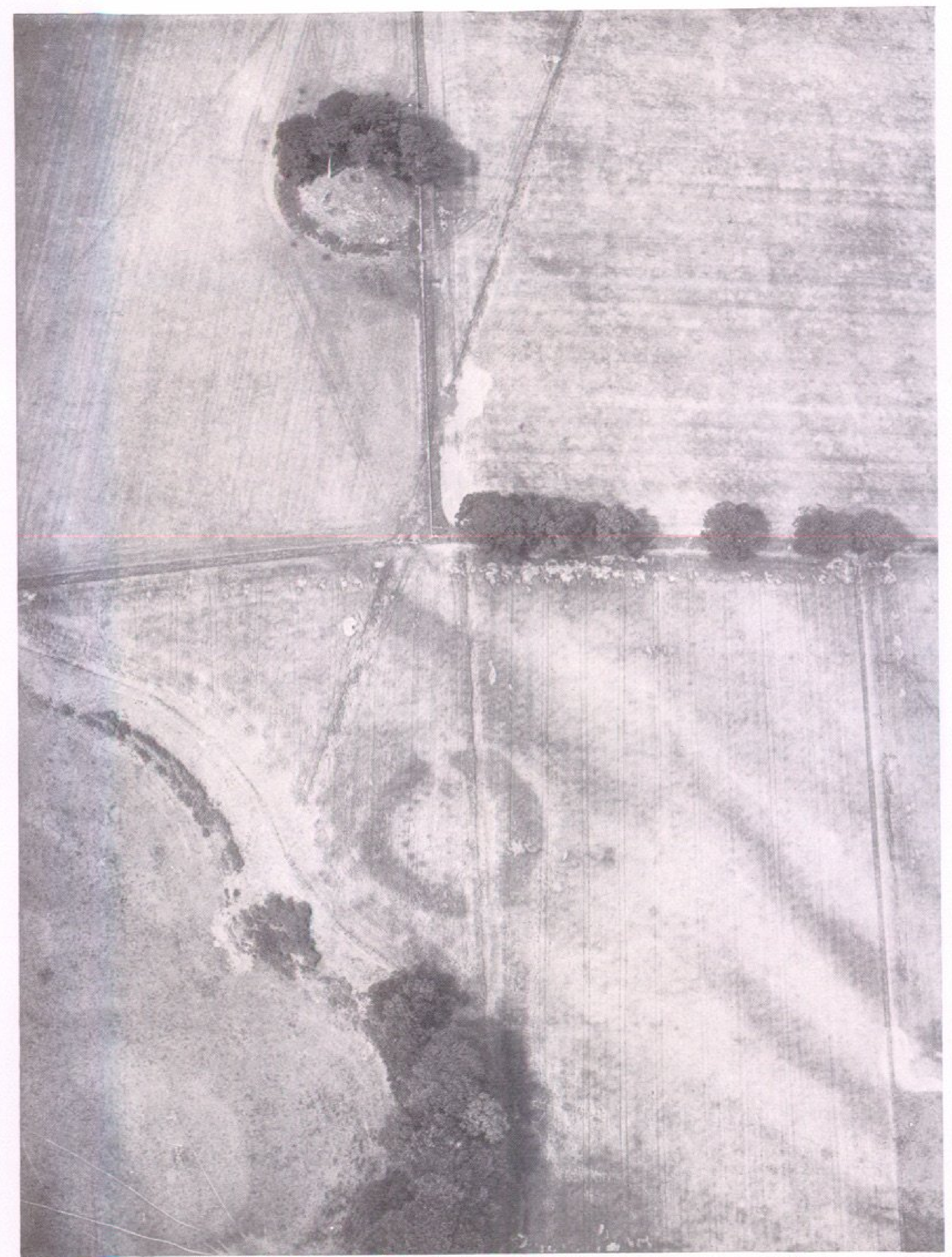

RCAMS aerial view (1977) from the E, showing the henge in the foreground, the barrow behind 
PLATE 10 | PSAS 113

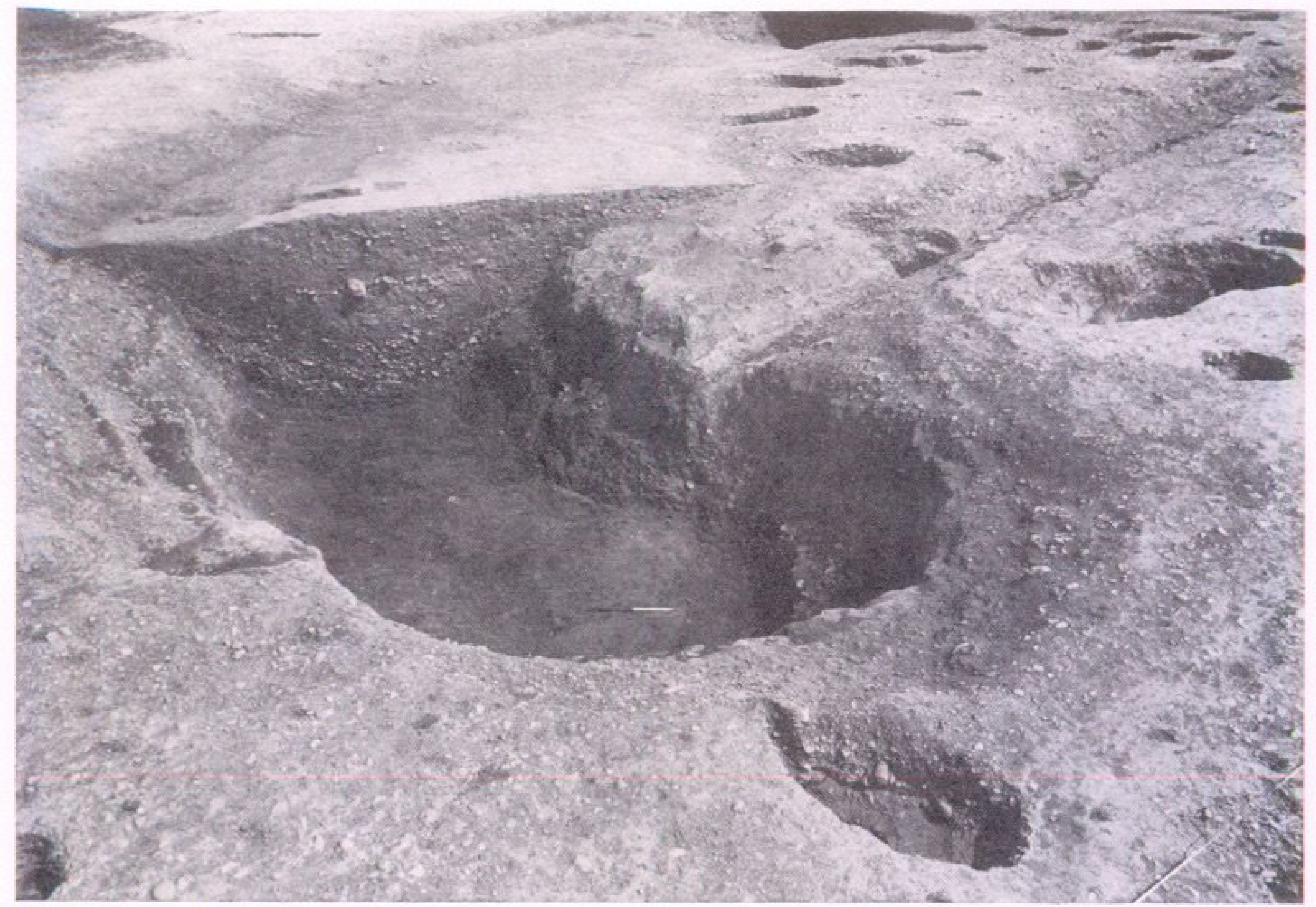

Henge: the NW ditch terminal

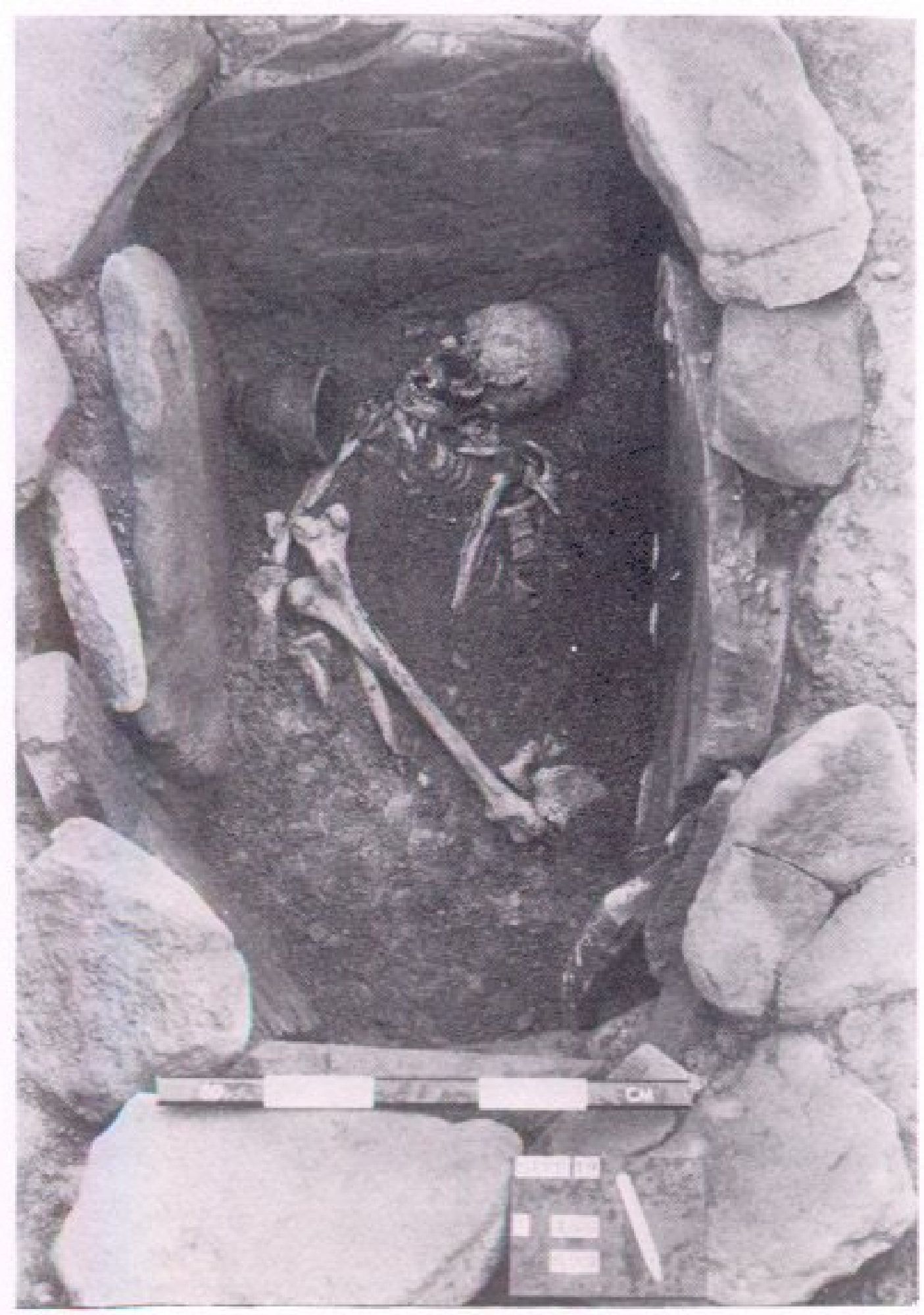

Henge: burial B

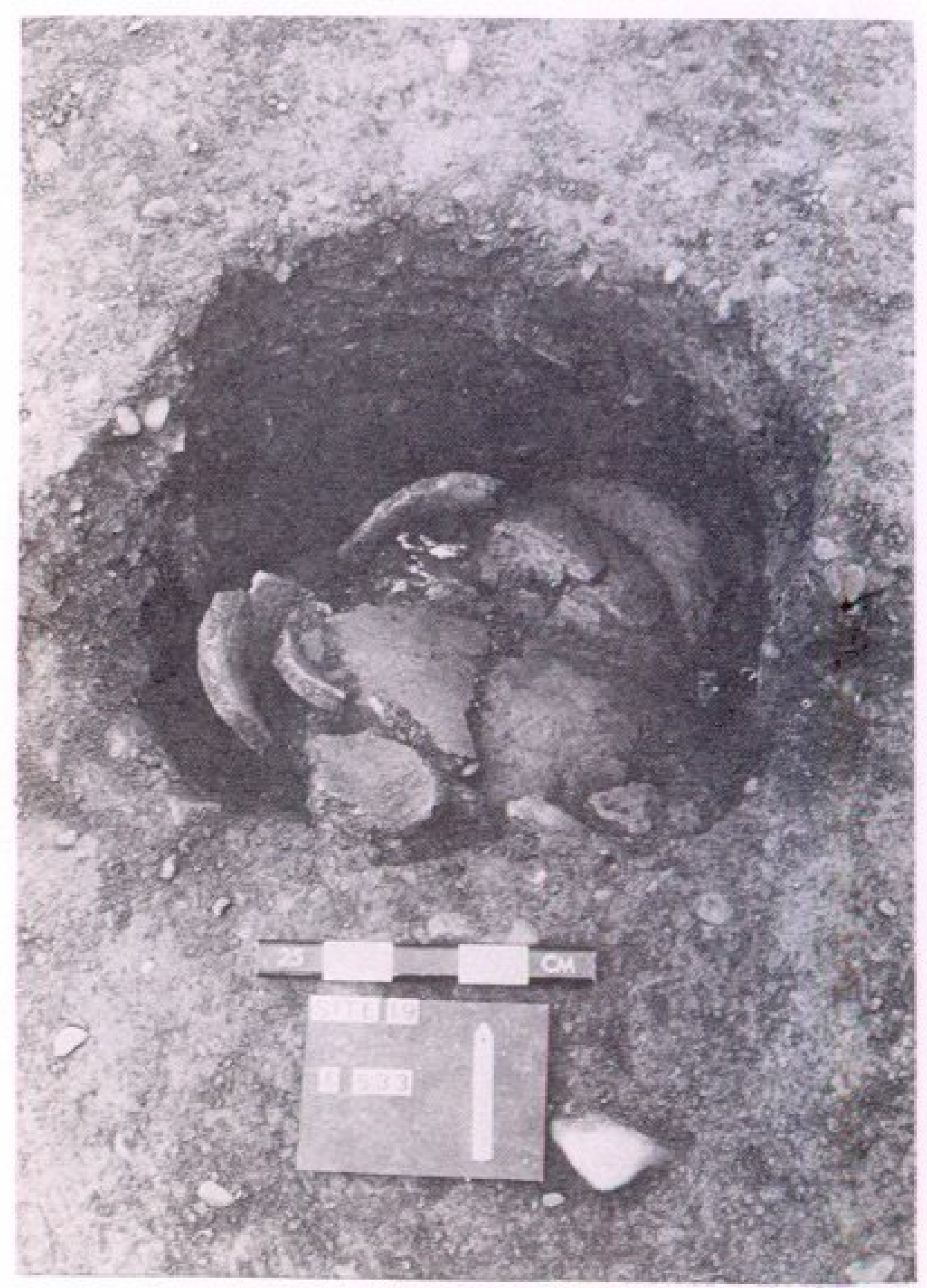

Henge: burial $\mathrm{H}$

BARCLAY | North Mains 


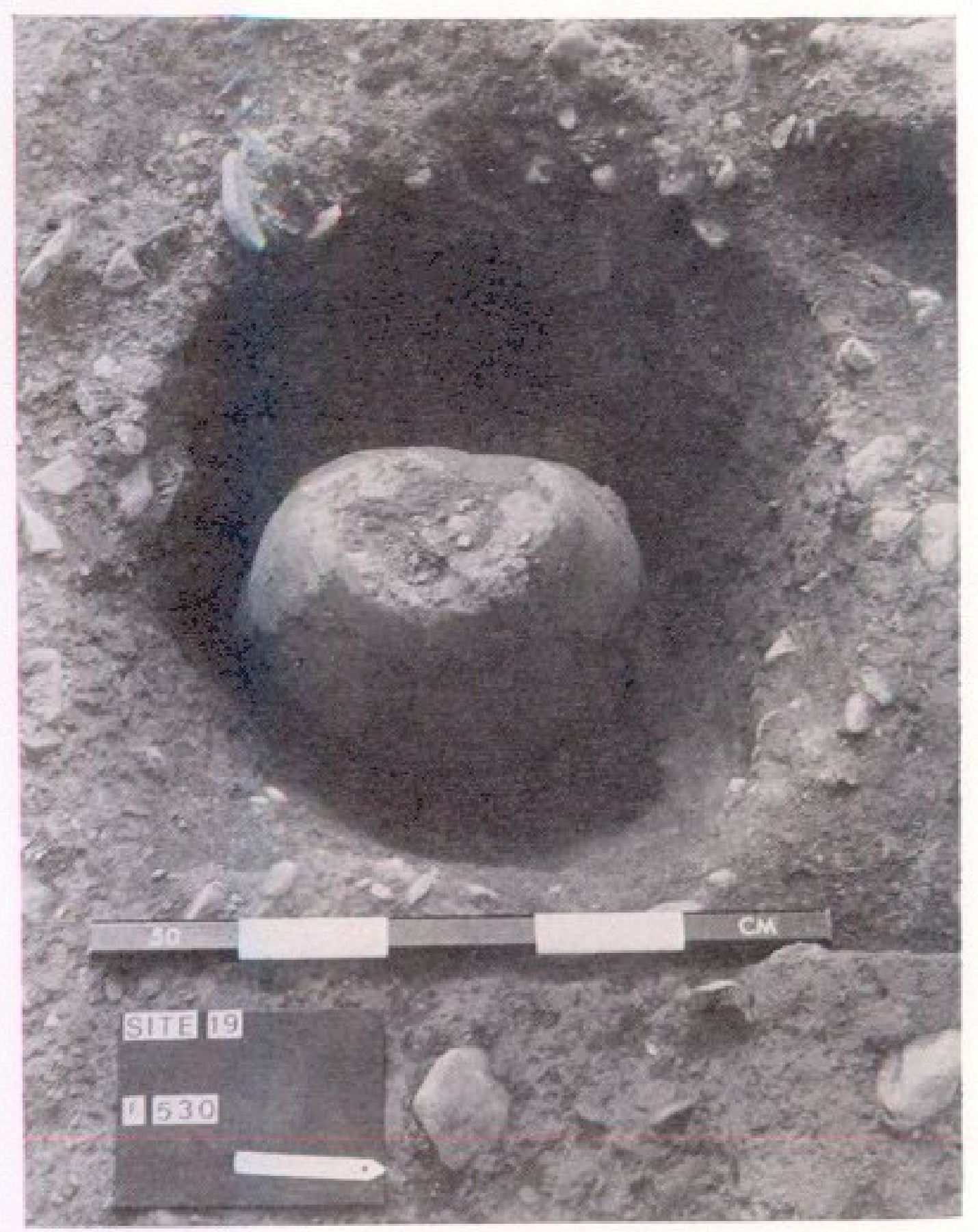

Henge: burial J

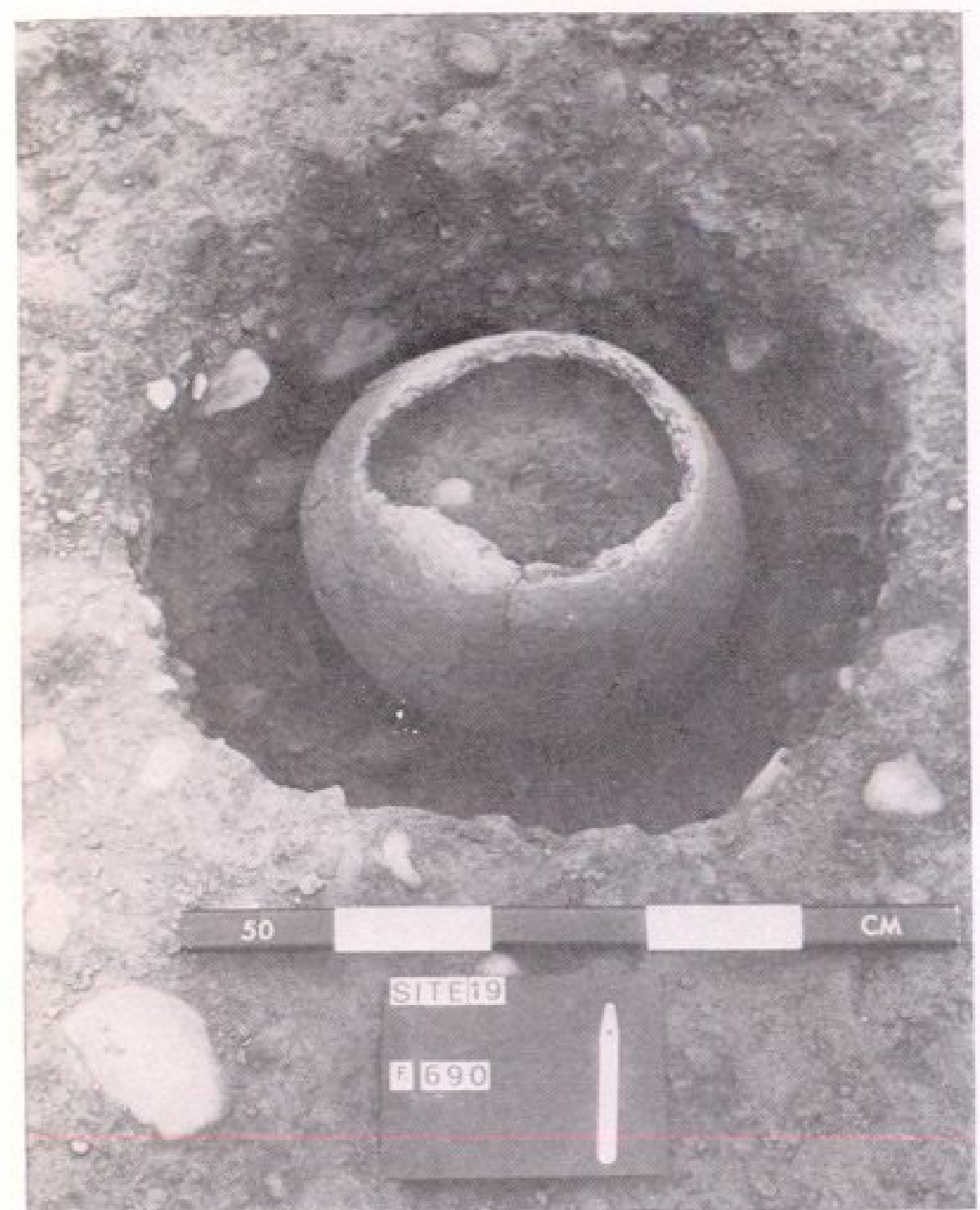

Henge: burial K

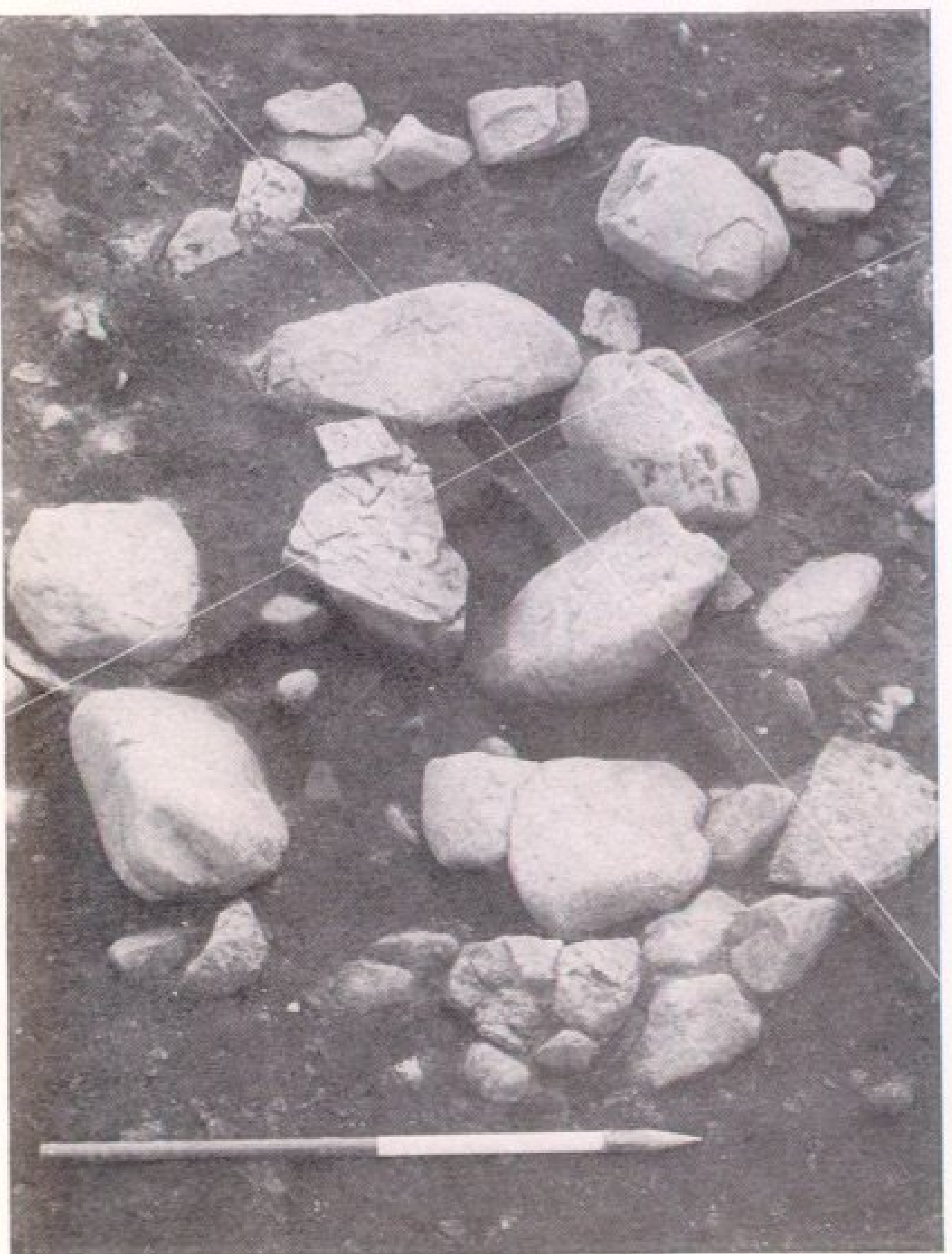

Henge: stone setting containing Burial $\mathrm{O}$ in the upper part of F6, to the $\mathrm{N}$ of the enclosure

North Mains | BARCLAY 


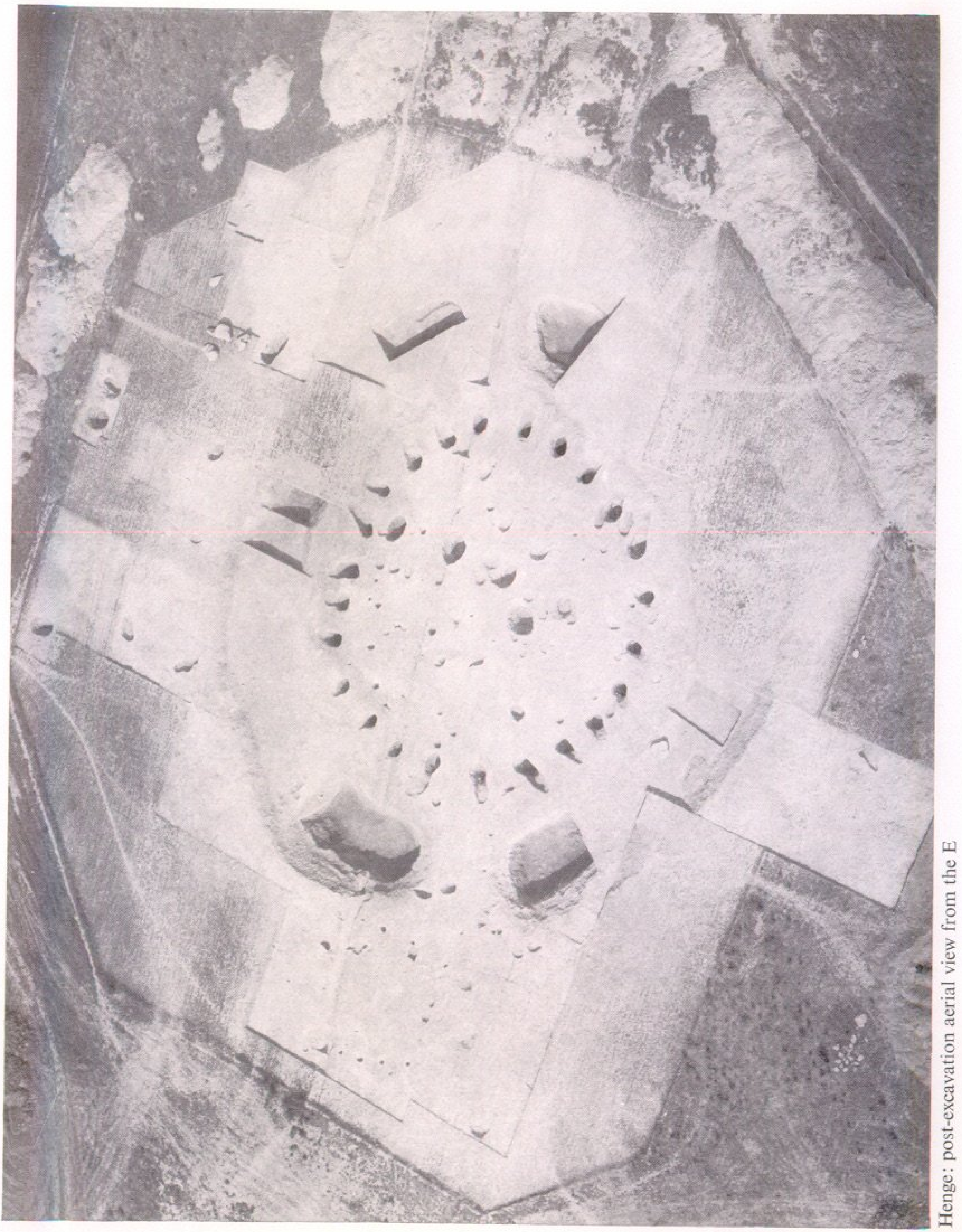




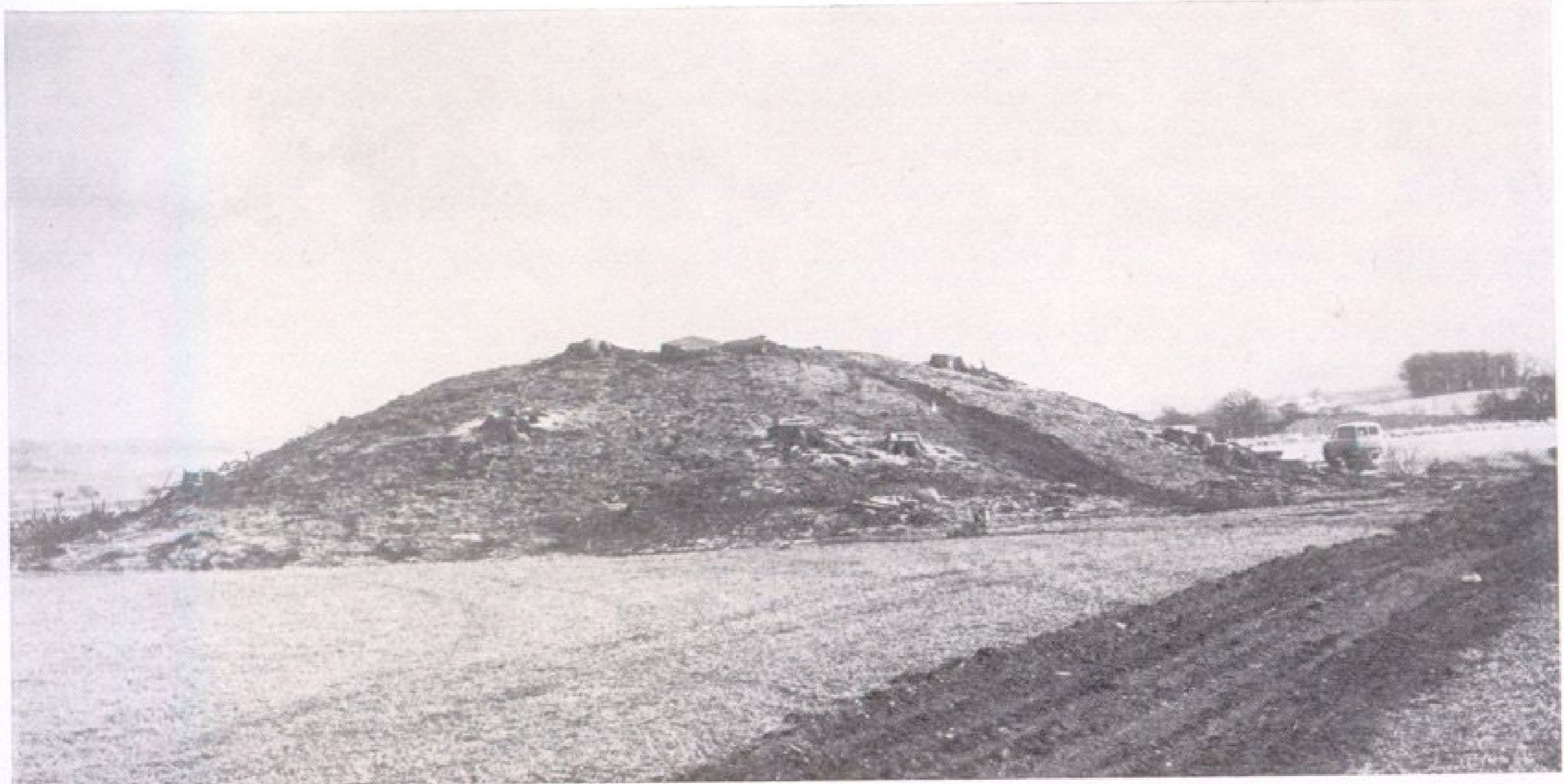

Barrow: prior to excavation, from the SW

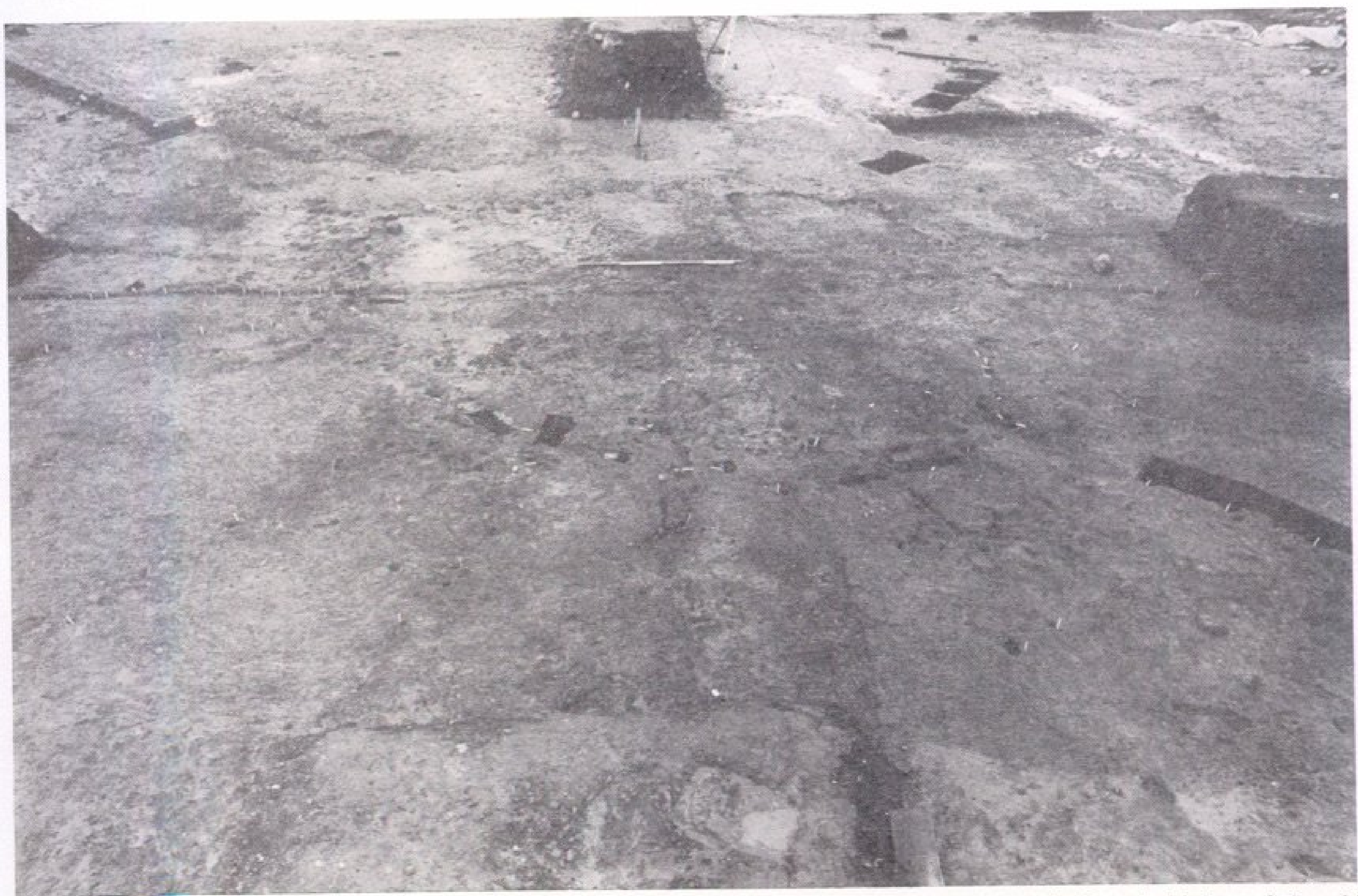

Barrow: the central enclosure on the old land surface. In the upper right corner the agricultural ridging of the old land surface can be seen 


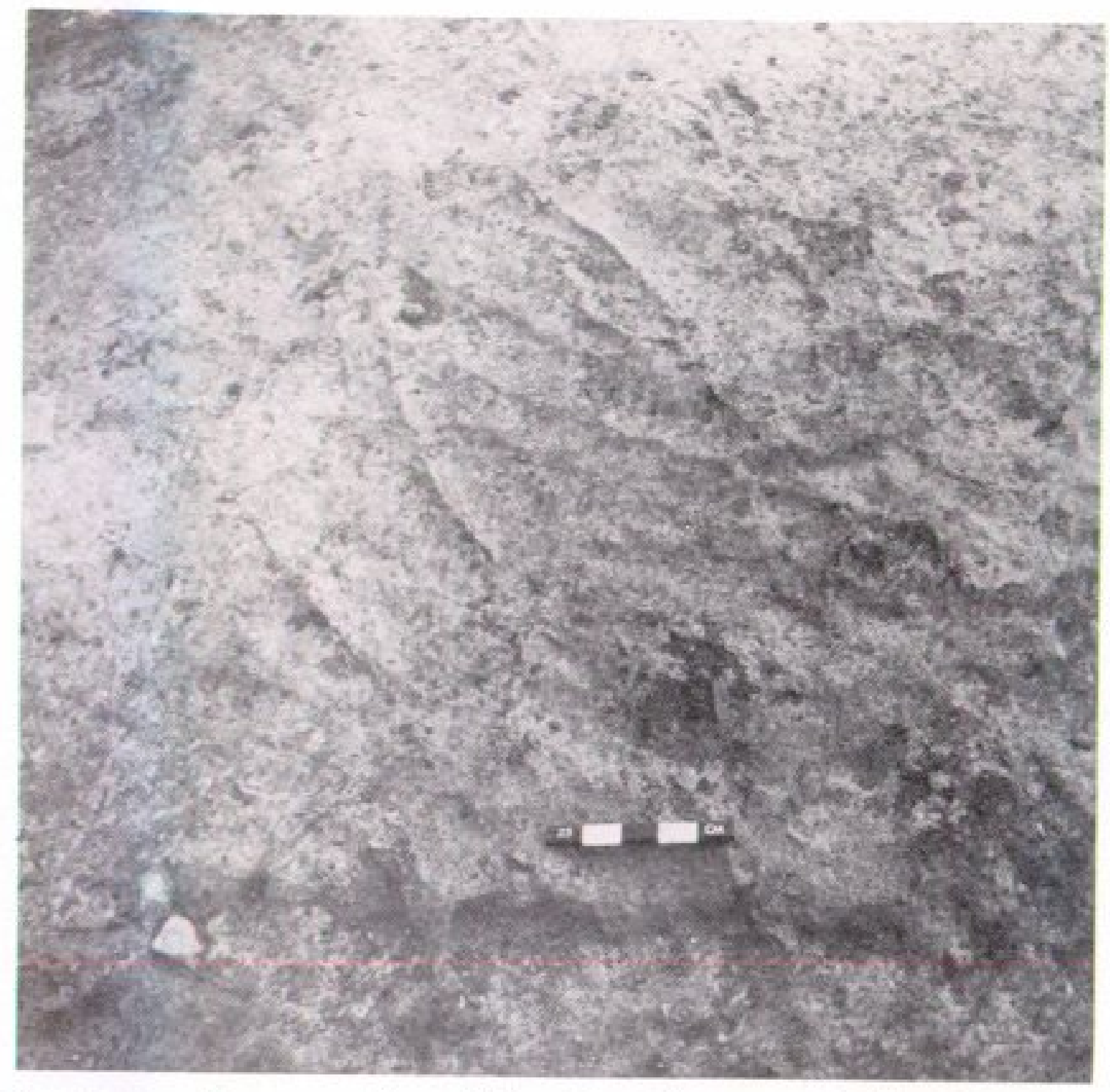

Barrow: ridges on the old land surface, probably showing the former position of pieces of wood

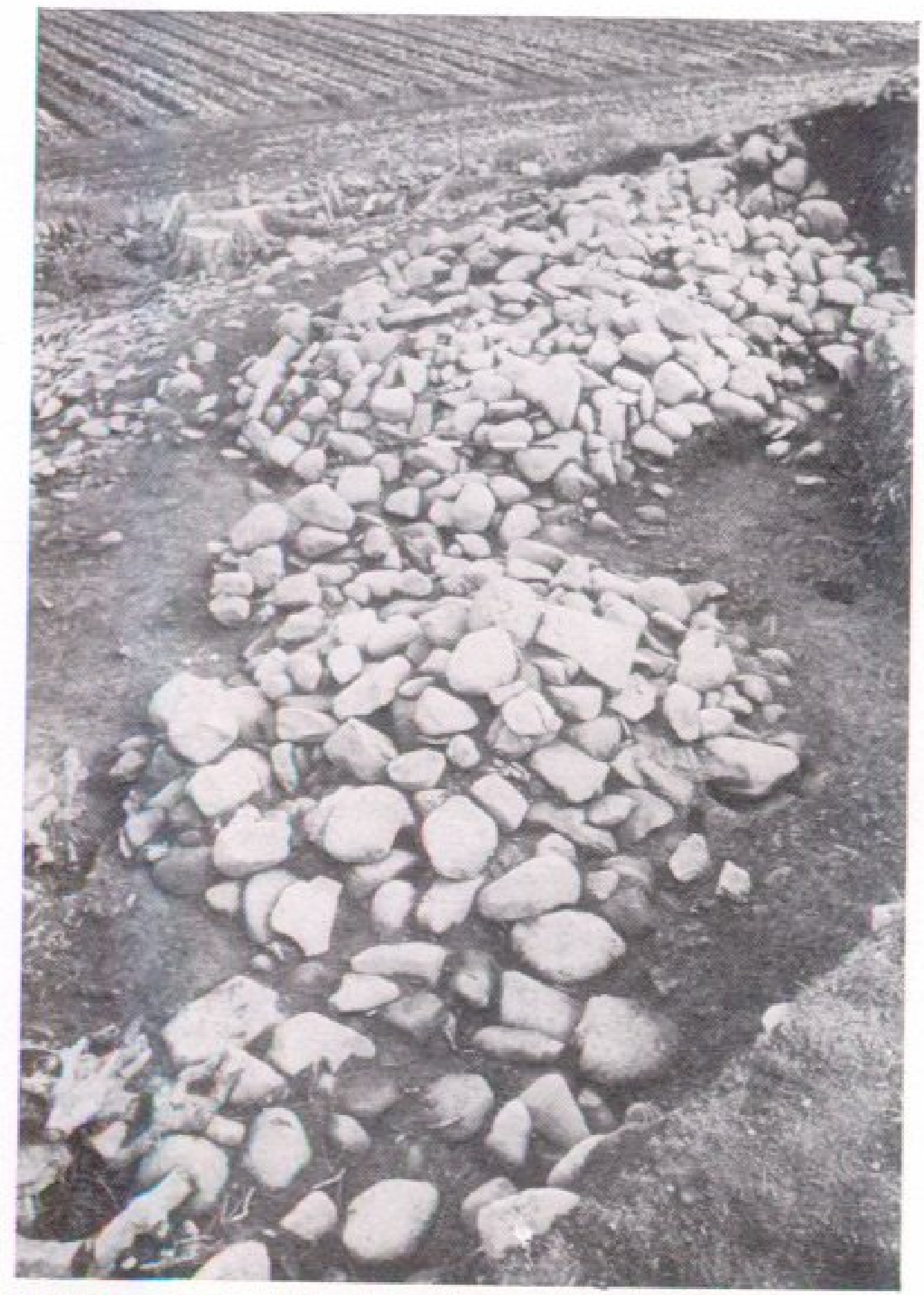

Barrow: part of the stone lens of Period III phase BC showing in plan in the NW quadrant

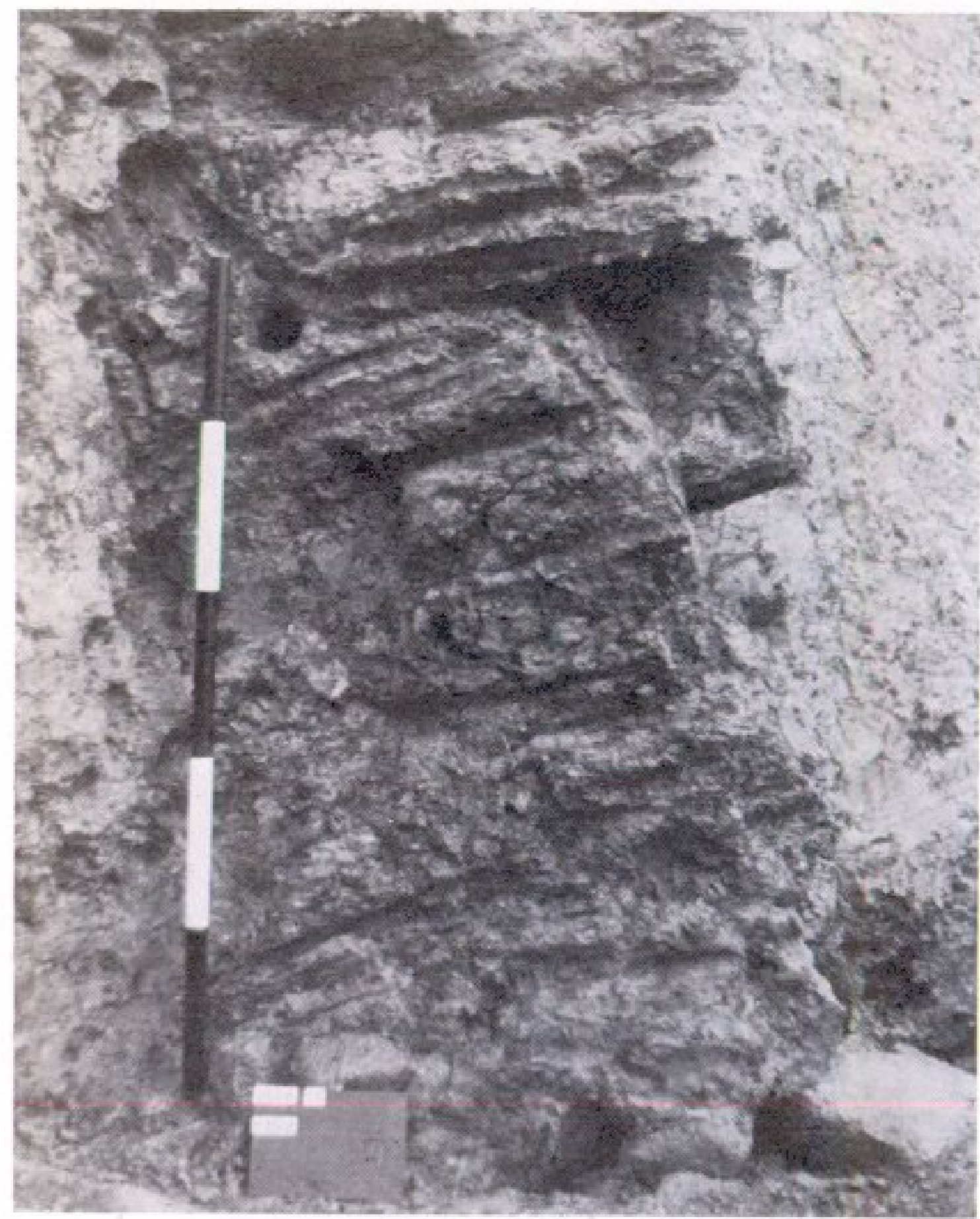

Barrow: impressions of fencing within the mound material in the SE quadrant

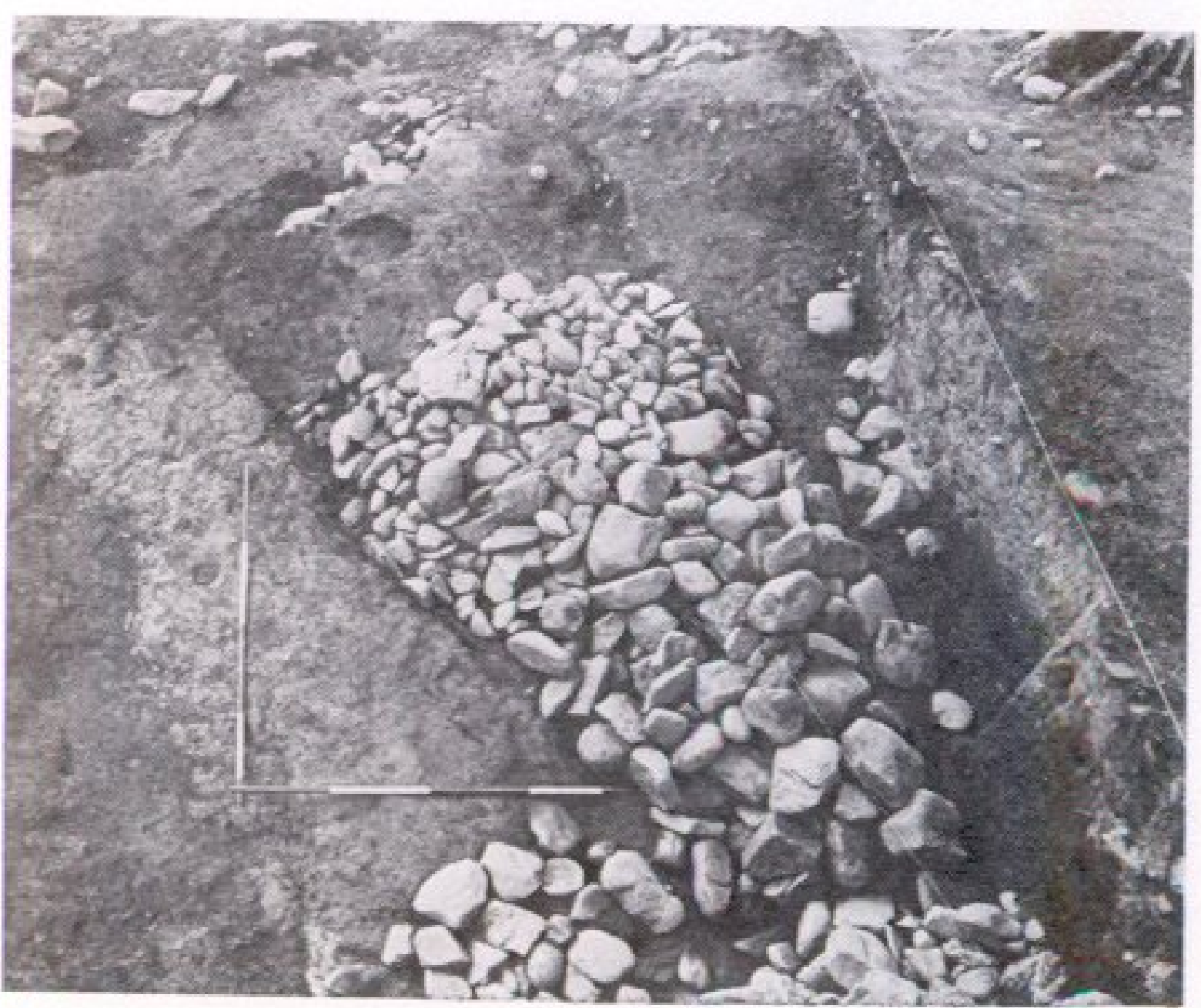

Barrow: the isolated stone dump high in the mound in the SE quadrant 
PSAS $113 \mid$ PLATE 15

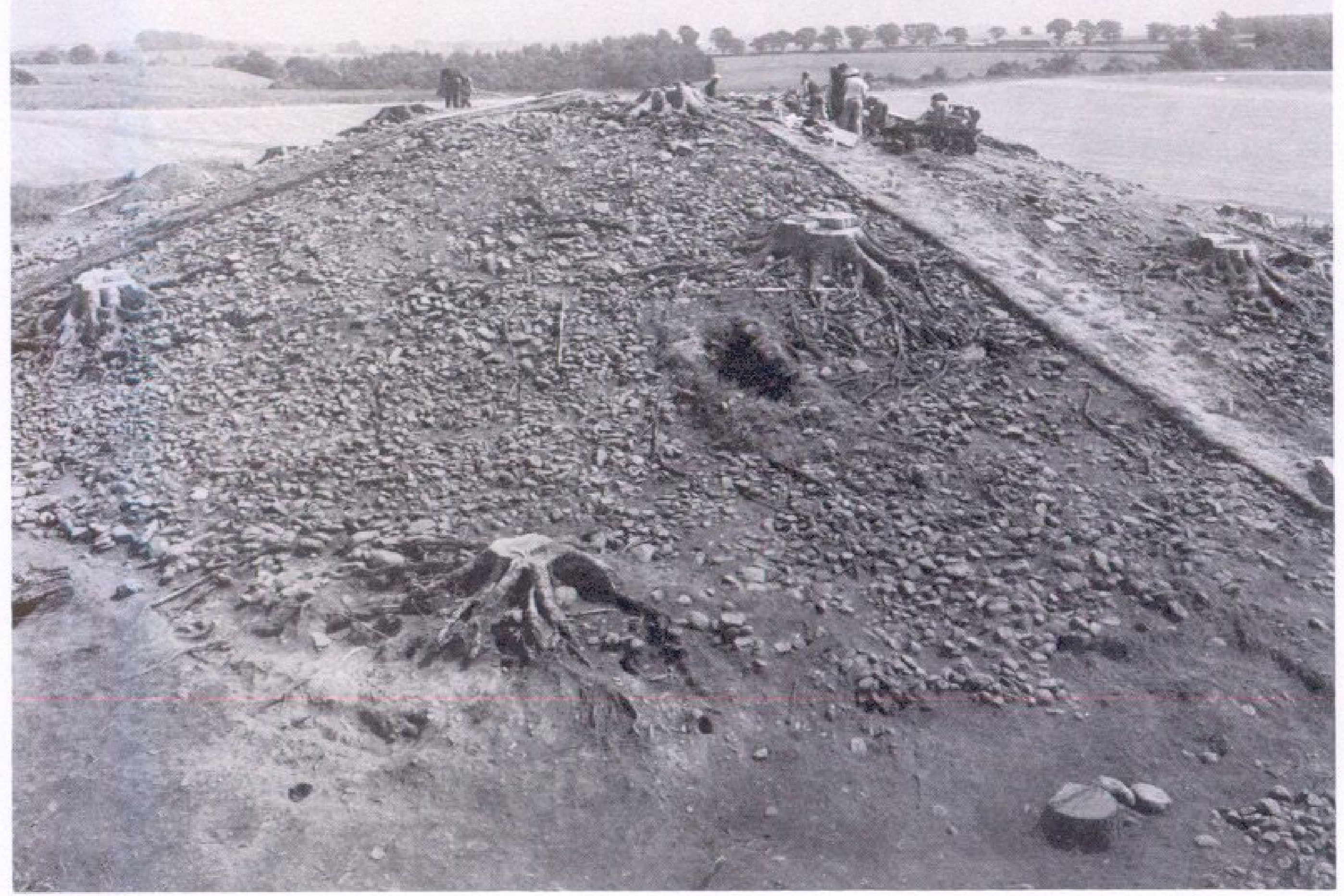

Barrow: the stone capping of the barrow, in the NW quadrant

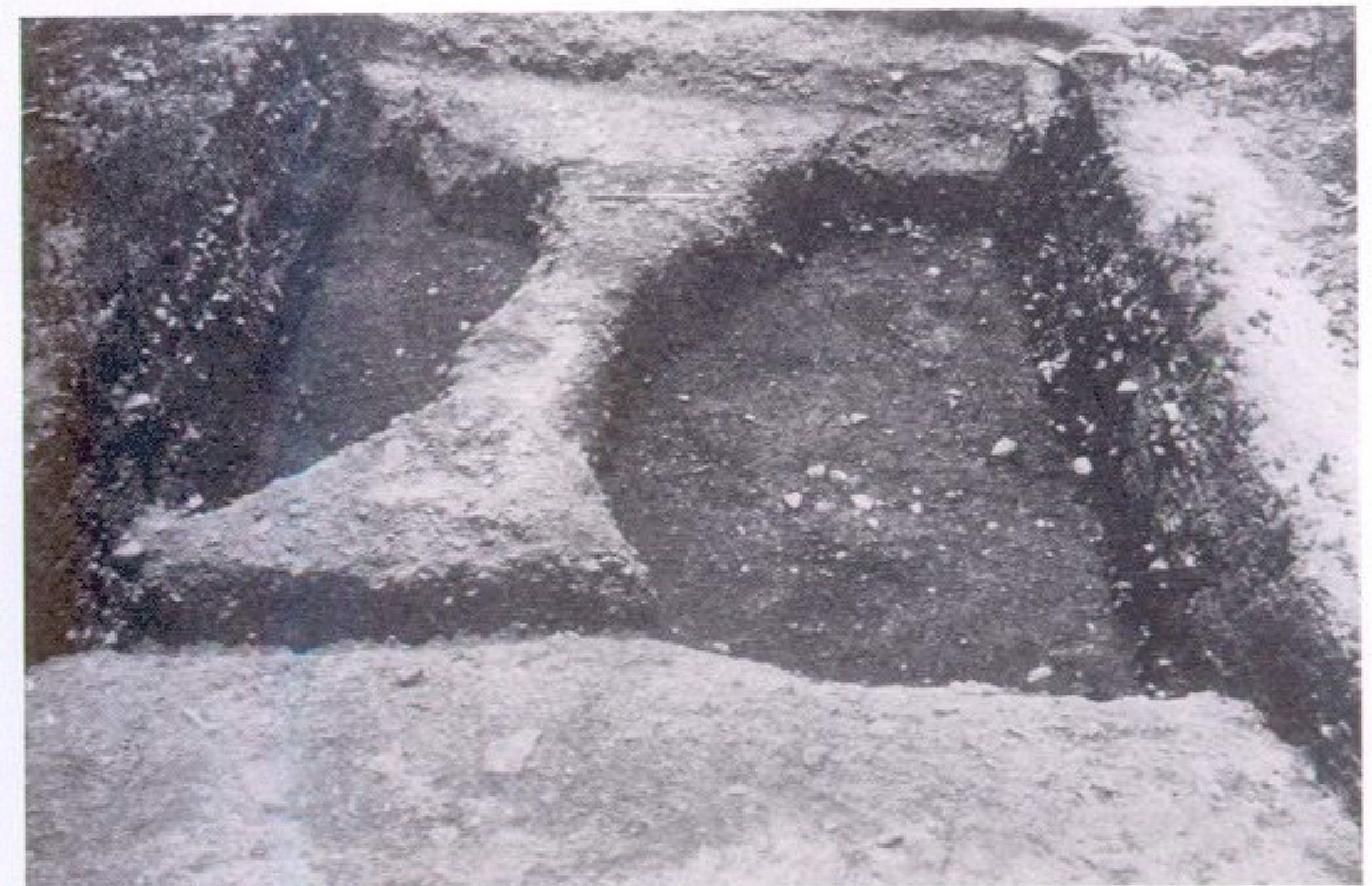

Barrow: the ditch at the NNE

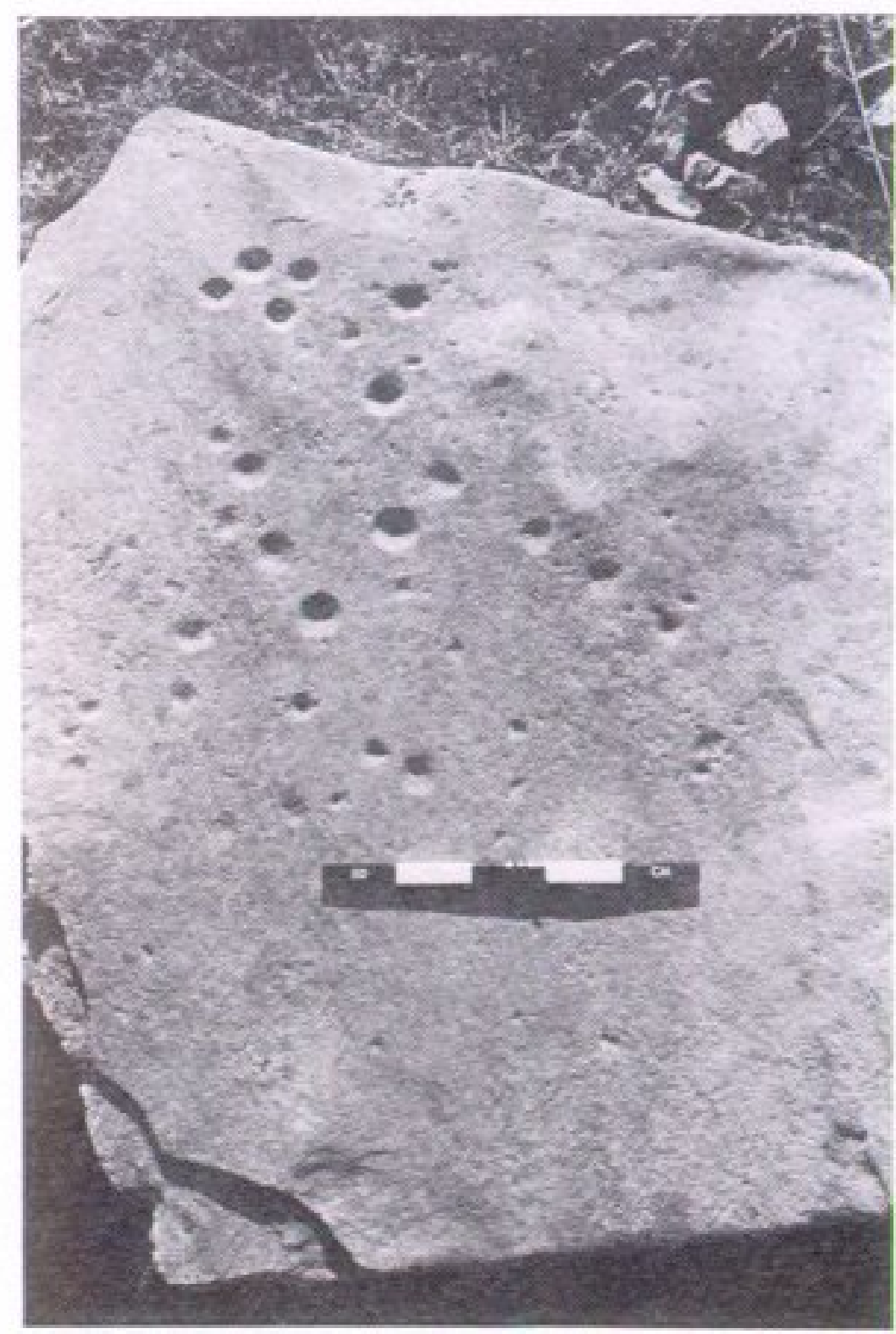

Barrow: the cupmarked stone as left at the summit by the excavators of the 1950 s 


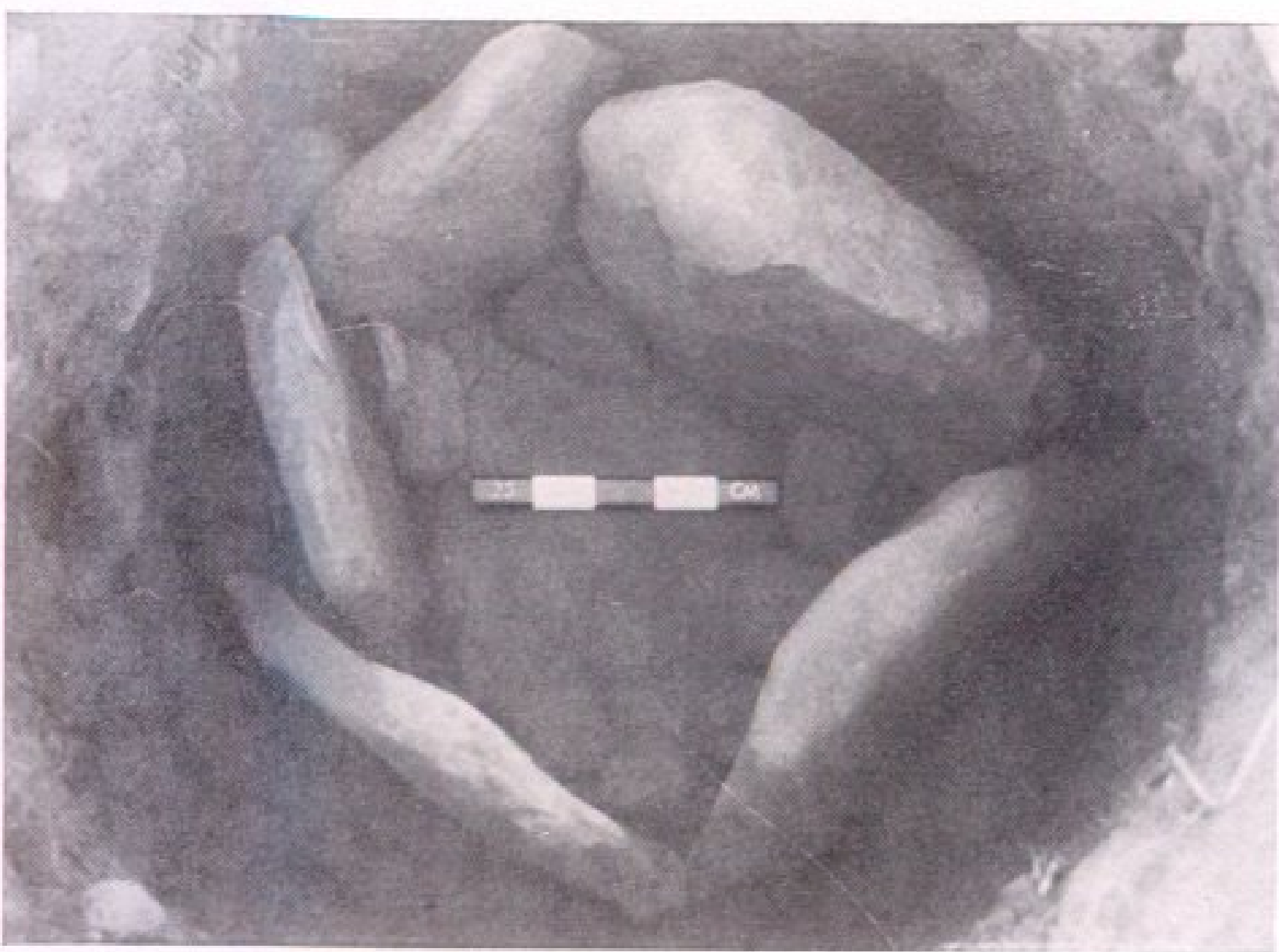

Barrow: the cupmarked slab forming the base of the cist containing burial $\mathrm{H}$

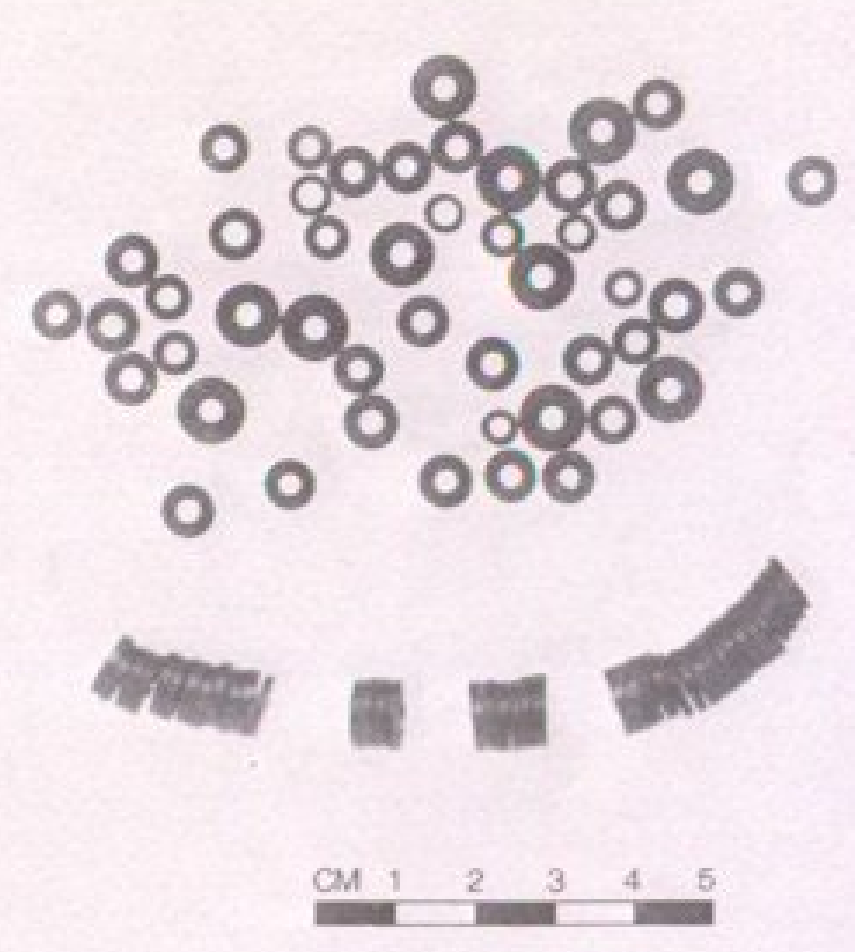

Barrow: the jet necklace (SF113), reconstructed as found

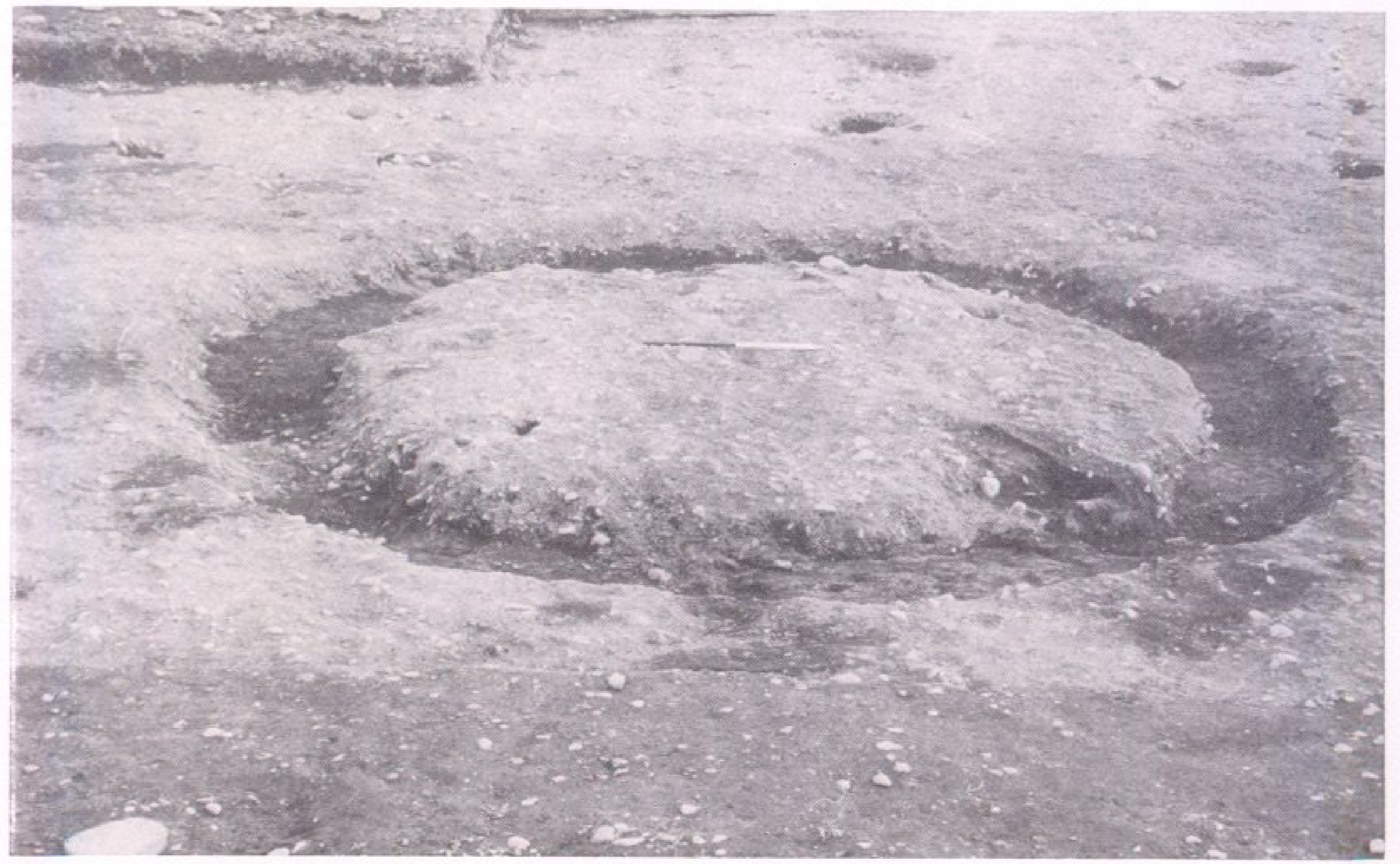

Ring-ditch 1: from the W 